\title{
Coherent X-Ray Diffractive Imaging on the Single-Cell-Level of Microbial Samples:
}

\section{Ptychography, Tomography, Nano-Diffraction and Waveguide-Imaging}

\author{
Dissertation \\ zur Erlangung des mathematisch-naturwissenschaftlichen Doktorgrades \\ Doctor rerum naturalium \\ der Georg-August-Universität Göttingen \\ im Promotionsprogramm PROPHYS \\ der Georg-August University School of Science (GAUSS)
}

vorgelegt von

Robin NikLas WiLkE

aus Bielefeld

Göttingen 2014 


\section{Betreuungsausschuss}

Prof. Dr. Tim Salditt

Institut für Röntgenphysik

Georg-August-Universität Göttingen

Prof. Dr. Claus Ropers

IV. Physikalisches Institut

Georg-August-Universität Göttingen

\section{Mitglieder der Prüfungskommission}

Referent: Prof. Dr. Tim Salditt

Institut für Röntgenphysik

Georg-August-Universität Göttingen

1. Korreferent: Prof Dr. Claus Ropers

IV. Physikalisches Institut

Georg-August-Universität Göttingen

\section{Weitere Mitglieder der Prüfungskommission}

Prof. Dr. Joachim Reitner

Institut für Geobiologie

Georg-August-Universität Göttingen

PD. Dr. Michael Hoppert

Institut für Mikrobiologie und Genetik

Georg-August-Universität Göttingen

Prof. Dr. Simone Techert

Institut für Röntgenphysik

Georg-August-Universität Göttingen

Prof. Dr. Jörg Enderlein

III. Physikalisches Institut

Georg-August-Universität Göttingen

Tag der mündlichen Prüfung: 20.10.2014 


\section{Contents}

\begin{tabular}{ll}
\hline Introduction & 1
\end{tabular}

$\begin{array}{ll}\text { Theory } & \mathbf{7}\end{array}$

1.1 Scalar Wave-Fields . . . . . . . . . . . . . . . . . 7

1.1.1 Propagation of Scalar Wave-Fields . . . . . . . . . . . . 7

1.1.2 Remarks on the Numerical Propagation of Scalar Wave-Fields 12

1.1.3 Partial Coherence . . . . . . . . . . . . . 15

1.2 X-Rays in Matter . . . . . . . . . . . . . . . . . . . 20

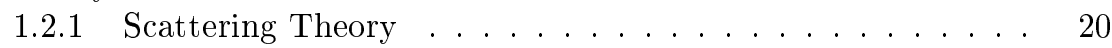

1.2.2 The Projection Approximation . . . . . . . . . . . . 24

1.2.3 Dose vs. Resolution . . . . . . . . . . . . . . . 30

1.3 Imaging with X-Rays . . . . . . . . . . . . . . . . . . . . . . . . . . . . . . 34

1.3.1 Direct Methods . . . . . . . . . . . . . . . 34

1.3.2 The Phase Problem . . . . . . . . . . . . . . . 35

1.3.3 Coherent Diffractive Imaging . . . . . . . . . . . . . . 36

1.3.4 Sampling in CDI . . . . . . . . . . . . . . . . . 37

1.3.5 Phase Retrieval Algorithms for Isolated Objects . . . . . . 39

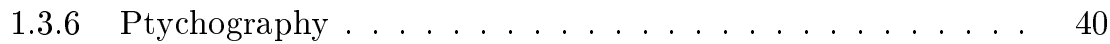

1.3.7 The ePIE algorithm . . . . . . . . . . . . . . . 41

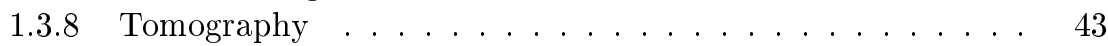

1.3.9 The Fourier Slice Theorem . . . . . . . . . . . . . . . 43

1.3.10 Filtered Back Projection Algorithm . . . . . . . . . . . . 43

$\begin{array}{ll}\text { Bacterial Samples } & \mathbf{4 7}\end{array}$

2.1 Deinococcus radiodurans . . . . . . . . . . . . . . . . . 47

2.2 Bacillus subtilis \& Bacillus thuringiensis. . . . . . . . . . . . 51

2.3 Sample Preparations . . . . . . . . . . . . . . . 55

2.3.1 Cell Culture of D. radiodurans . . . . . . . . . . . 55

2.3.2 Cell Culture of B. subtilis \& B. thuringiensis . . . . . . . . 57

2.3.3 Preparation of Ultra-thin Sections for Transmission Electron

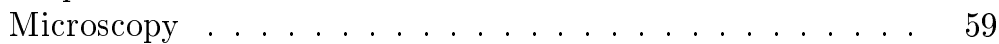

2.3.4 Vitrification . . . . . . . . . . . . . 60

2.3.5 Lyophilisation. . . . . . . . . . . . . 63

$\begin{array}{ll}\text { Experiments } & 69\end{array}$

3.1 Ptychographic Imaging and Nano-Diffraction of D. radiodurans . . 69

3.1.1 Experimental Set-up . . . . . . . . . . . . . . 70

3.1.2 Sample Preparation . . . . . . . . . . . . . 71

3.1.3 Details and Terminology: Cellular Nano-Diffraction . . . . 71

3.1.4 Results: Cellular Nano-Diffraction . . . . . . . . . . 72

3.1.5 Details and Theory: Ptychographic Imaging . . . . . . . . . 79

3.1.6 Details: Tomographic Reconstruction . . . . . . . . . 82 
3.1.7 Results: Ptychographic Imaging of the Resolution Chart and Wave-Field Analysis . . . . . . . . . . . . . 83

3.1.8 Resolution vs. Coherence . . . . . . . . . . . . . . . 94

3.1.9 Results: Ptychographic Imaging Towards a 3D Representation of D. radiodurans Cells . . . . . . . . . . . . . . . . . . 97

3.1.10 Results: Tomo-Ptychographic Imaging of D. radiodurans cells 100

3.1.11 Results: TEM Analysis of D. radiodurans . . . . . . . . . . 103

3.1.12 Discussion \& Conclusion . . . . . . . . . . . . . 107

3.2 Scanning Hard X-ray Microscopy Imaging Modalities for Geobio-

logical Samples . . . . . . . . . . . . . . . . . . 110

3.2.1 Sample Origin \& Sample Preparations . . . . . . . . . . . . 111

3.2.2 Details: Scanning X-Ray Fluorescence and Scanning Transmission X-Ray Experiments . . . . . . . . . . . . . . . 111

3.2.3 Details: Scanning Transmission X-Ray and X-Ray Ptychog-

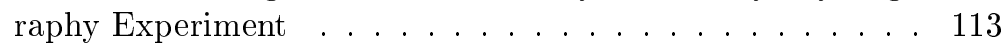

3.2 .4 Results ....................... 119

3.2.5 Details \& Results: SEM-EDX Analysis . . . . . . . . . . . . 126

3.2 .6 Discussion \& Conclusion . . . . . . . . . . . . . . . . 129

3.3 STCS in High-Resolution X-Ray Ptychography Using KB Focusing 132

3.3.1 Experimental Set-up . . . . . . . . . . . . . 133

3.3 .2 Details: Analysis . . . . . . . . . . . . . . . 136

3.3.3 Results \& Discussion . . . . . . . . . . . . . . . . . . 138

3.3.4 Summary \& Conclusion . . . . . . . . . . . . . . . . 142

3.4 An STCS Application in High-Resolution X-Ray Ptychography . . 144

3.4.1 Experimental Set-up . . . . . . . . . . . . 145

3.4.2 Details: STCS Preparations . . . . . . . . . . . . . . . 147

3.4.3 Samples . . . . . . . . . . . . . . . . . 147

3.4.4 Details: Data Recording \& Data Treatment . . . . . . . . . 148

3.4.5 Results: Ptychographic Reconstruction of KB Wave-Fields 149

3.4.6 Results: Ptychographic Imaging of Nanowires . . . . . . . . 153

3.4.7 Discussion, Conclusion \& Outlook . . . . . . . . . . . . 155

3.5 Waveguide-Based Imaging of B. thuringiensis and B. subtilis . . . 161

3.5.1 Experimental Set-up . . . . . . . . . . . . . . 161

3.5.2 Sample Preparation . . . . . . . . . . . . . . 162

3.5.3 Details: Theory, Data Recording \& Data Treatment . . . . 164

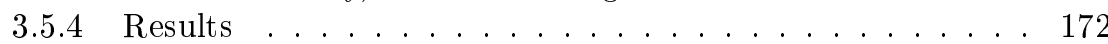

3.5.5 Conclusion, Discussion \& Outlook . . . . . . . . . . 185

4 Summary, Conclusion \& Outlook 187

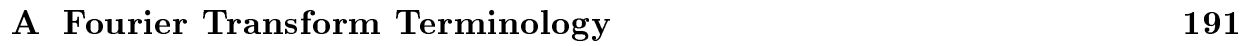

B Culture Media 192

\begin{tabular}{ll|} 
C EM Protocols & 192
\end{tabular} 


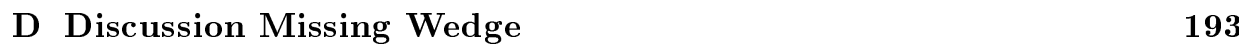

E ePIE Amplitude Reconstruction $\quad 195$

$\begin{array}{lll}\text { F } & \text { TEM Images } & 196\end{array}$

\begin{tabular}{ll}
\hline G STCS List & 197
\end{tabular}

H Algorithmic Parameters for Waveguide-Based Imaging 198

$\begin{array}{llr}\text { I Publications } & 229\end{array}$

$\begin{array}{lr}\text { Publications } & 229\end{array}$

Danksagung - Acknowledgements 233

$\begin{array}{ll}\text { Index } & 235\end{array}$ 


\section{Nomenclature}

A. willeyana

B. subtilis

B. thuringiensis

$D$. radiodurans

BG

CDI

cSAXS

DAPI

DESY

df

DFT

DM

DNA

DOF

DPC

DSMZ
DIC

Astrosclera willeyana

Bacillus subtilis

Bacillus thuringiensis

Deinococcus radiodurans

Background

Coherent Diffractive Imaging

coherent Small Angle X-Ray Scattering

4',6-Diamidino-2-phenylindole dihydrochloride

Deutsches Elektronen-Synchrotron

Darkfield contrast

Discrete Fourier transform

Differential interference contrast

Difference-Map

Deoxyribonucleic acid

Depth of focus

Differential phase contrast

Deutsche Sammlung von Mikroorganismen und

Zellkulturen

EDX

EM

ePIE

ER

ESRF

FFT

FOV

FST

FWHM

FZP

GINIX

GS

$\mathrm{KB}$

LB

mHIO

PBS

PCDI

PETRA

PRTF

PSD

PSI

SAXS

SEM

SLS
Energy Dispersive X-ray spectroscopy

Electron Microscopy

Extended Ptychographic Iterative Engine

Error-Reduction

European Synchrotron Radiation Facility

Fast Fourier transform

Field of view

Fourier Slice Theorem

Full width at half maximum

Fresnel zone plate

Göttingen Instrument for Nano-Imaging with Xrays

Gerchberg-Saxton

Kirkpatrick-Baez

Lysogeny broth

modified Hybrid-Input-Output

Phosphate Buffered Saline

Ptychographic Coherent Diffractive Imaging 'Positron-Electron Tandem Ring Accelerator' Phase retrieval transfer function

Power spectral density

Paul Scherrer Institut

Small Angle X-Ray Scattering

Scanning Electron Microscopy

Swiss Light Source 
SOX $\ldots \ldots \ldots \ldots \ldots \ldots \ldots$ Sulfur-oxidizing bacteria

spp. .................. species pluralis

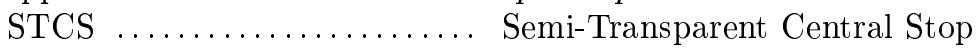

STXM $\ldots \ldots \ldots \ldots \ldots \ldots \ldots$ Scanning Transmission X-Ray Microscopy

TEM $\ldots \ldots \ldots \ldots \ldots \ldots \ldots$ Transmission Electron Microscopy 



\section{Introduction}

X-rays and electrons are examples of powerful, complementary and well-established probes of nanostructures. In case of X-rays, biological imaging is usually performed in the soft X-ray region. In particular, the 'water window' between photon energies of about $200 \mathrm{eV}$ and $700 \mathrm{eV}$ allows for a natural contrast enhancement between carbon and water due to absorption edges $[50,161,167,219,256,290$. In the ideal case, biological specimens are studied in vivo, in their native, living state which usually excludes any preceding invasive sample treatments and working in a vacuum environment. The use of hard X-rays can be advantageous for several reasons. For instance, the weak interaction of X-rays of higher energy with matter allows for probing the three-dimensional structure of comparably thick specimens and the sample environment can be relaxed to ex-vacuum conditions. Importantly, X-rays provide many different possible imaging modalities which alleviates requirements for sample staining and the use of contrast agents. For instance, X-ray phase contrast is a quantitative measure of the electron density. In addition, one can take advantage of absorption edges in order to obtain an elemental speciation of the sample. Complementary to these real-space imaging modes, the high energy facilitates recording of small angle diffraction signals that hold high-resolution structural information about the sample in reciprocal space. Notwithstanding the ongoing progress in development of X-ray optics, detectors and methodology, the achieved imaging resolution stands behind the expectation. That is, the obtained resolution is increasing but still well above the wavelength (cf. |133, 266, 314). Currently, there is thus a great potential for improvement of probing micron-sized biological samples on the nanometre-scale in the hard X-ray region.

Suitable, micron-sized biological sample systems for X-ray imaging experiments are given by microbial specimens whose fascinating life is driven by their structure on the nanometre-scale. Microorganisms have ever been strongly entangled with the life on earth since the begin of evolution of microbial life dating back to about 3.8 billion years ago 57 . An important example is the oxygenation of the atmosphere by oxygenic phototrophs which paved the way for more complex cellular life-forms. In addition, microbial life has got a great impact on many different important facets of our life such as food and agriculture (digestion and fermentation of cellulose, methanogenesis, nitrogen fixation, insecticides) [64, 132, 206, 263, 291], pharmaceutical and chemical industry (protein production, biofuels, microbial bioremediation) $[3,93,121,162,254]$ and health in general due to the pathongenicity of some species and food spoilage (cf. $\mid 180$ ).

Among eukarya and archaea, bacteria including the well-known Escherichia coli form one prominent domain of single-celled, microbial life. On a comparably large scale, the constitution of bacterial cells is fairly simple. There is only the cytoplasma containing e.g. proteins, lipids, nucleic acids and ribosomes which is enclosed and protected from the outside by the cytoplasmic membrane and the cell wall. If one defines 'local ordering' as a repetitive structure that can be described by significant Fourier components one finds the organisation of the bacterial cell to be comparably unordered on a spatial scale above about $10 \mathrm{~nm}$. Below this scale 
one can find surprisingly many important cellular structures which exhibit a high degree of local ordering. For instance, methanotrophs use highly ordered internal stacks of membranes for methane oxidation (cf. Fig. 1). Considering examples of microbial samples being addressed within this thesis, there is also the finestructure of the nucleoid of the bacterium Deinococcus radiodurans that may be linked to its outstanding resistance to ionizing radiation $[77]$ and the highly ordered S-layer which is common amongst other bacteria 253, 278, 279, too. In case of the endospore forming bacterium Bacillus thuringiensis, one finds the honeycomb structure of the endospore coat $[231,232]$. Moreover, B. thuringiensis is well known for its ability to produce intracellular protein crystals $[2,9,132,263$.

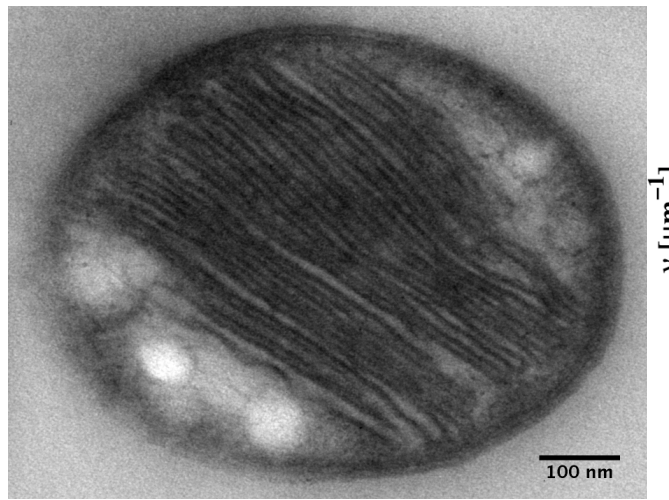

(a)

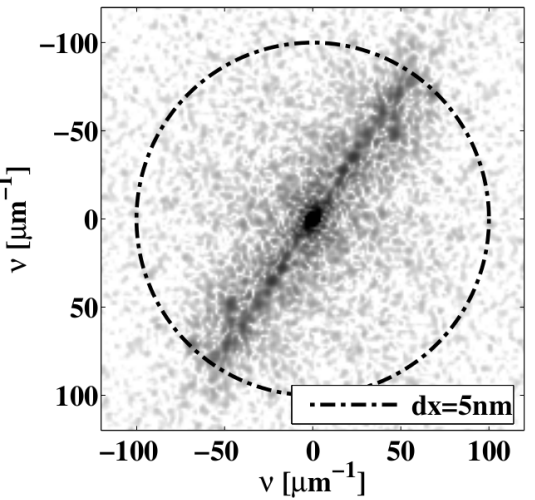

(b)

Figure 1: The figure illustrates the local ordering of the stacks of membranes that can be found within methanotrophs. (a) shows a transmission electron microscopy image of a resin-embedded, chemically fixed and stained ultra-thin section of a single cell. (b) shows the calculated power spectral density of the TEM image on logarithmic scale. The ordering of the membranes appears as a dark streak in the Fourier spectrum which extends down to a real-space length of about $5 \mathrm{~nm}$ (i.e. the resolution of the TEM image).

Due to coherent interference, X-ray diffraction experiments can be highly sensitive to structures expressing local ordering on such small length scales. However, the local ordering of all these cellular structures is not always as perfect as the structure of single crystals. One thus expects probing such structures with X-rays to be challenging from a point of view of the level of ordering and due to the fact that they consist of soft, weakly scattering biological matter that is prone to radiation damage. In addition, the ordering is restricted to small regions of the bacterial sample implying that the scattering contributions can be expected to be highly position dependent when illuminated with a sufficiently small X-ray beam. On larger samples such as wood, bone, alloys and muscles, scanning small angle X-ray scattering (SAXS) experiments have already been successfully carried out using micron-sized beams $\mid 29,87,88,106,157,171,224,248,318$. Due to the technological 
improvements of X-ray focusing optics also beam sizes well below one micron have been established (e.g. 65, 130, 200, 201, 264, 266, 313, 314 ). Scanning SAXS experiments using these nano-beams are ideally suited to analyse melanosomes and eukaryotic cells, even in a fully hydrated and living state |104 105 239,240 322 323]. On the other hand, the increasing availability of coherent hard X-ray nano-beams has opened up the experimental window for Coherent Diffractive Imaging (CDI) techniques which can be used to obtain electron density maps at unprecedented real-space resolution $1,41,196,197$. In contrast to standard direct imaging procedures, CDI relies on iterative algorithms that reconstruct the image information based on a priori constraints without the need of an image-forming lens.

The principal CDI method that was applied in this thesis is termed ptychography $250,251,296$. The ptychographic imaging scheme relies on scanning the probed object with a small beam. Hence, ptychography can be ideally combined with other X-ray scanning methods like SAXS or X-ray fluorescence. It originates from the field of electron microscopy $\mid 136,138,139,210$. The great power of the ptychographic method is its capability to simultaneously reconstruct not only information about the probed object but also about the illuminating wave-field which typically is denoted as the probe $183,294,296$. In principle, the capability to separate the illuminating wave-field from the object makes the retrieved object information free of aberrations. If the experimental conditions are sufficiently good ptychography has even proven to yield quantitative phase maps with a resolution of the object information below $10 \mathrm{~nm}$ in two dimensions [266, 287. and around $16 \mathrm{~nm}$ in three dimensions [133]. Recently, ptychography could also be shown to yield information under non-ideal experimental conditions such as a low degree of spatial and temporal coherence of the illuminating wave-field $\mid 78,299$ but also vibrational modes of the object could be extracted [42]. In case of unstained biological samples, quantitative two-dimensional images of frozen-hydrated yeast cells [172], freeze-dried cells of the bacterium Deinococcus radiodurans 97 and a three-dimensional representation of a mouse femur [61] were obtained before or during the time of this thesis (more ptychographic applications are listed in section 1.3 .6 , p. 40).

The central goal of this thesis was an improvement of the ptychographic imaging capabilities of the Göttingen Instrument for Nano-Imaging with X-rays (GINIX; cf. [154]) at the P10 beamline of the PETRA III light source with a possible application to microbial specimens on the level of single cells. In order to reach this goal, the focus of this work was split into three main branches that were pursued intensively. First, ptychographic imaging experiments were performed on the scale of single bacterial cells D. radiodurans at the coherent SAXS (cSAXS) beamline of the Swiss Light Source (SLS) providing excellent experimental capabilities both for scanning SAXS and ptychography. In parallel, the second branch consisted of ptychographic imaging experiments with an emphasis on exploiting the full flux capabilities of the GINIX. The final branch aimed at exploring new bacterial sample systems extending the landscape of sample systems that were initially available in the laboratory of T. Salditt. 
The thesis is organised as follows. In the first section, 'Theory', the theoretical concepts of quantitative X-ray phase contrast imaging are reviewed. This includes propagation of X-ray wave-fields, interaction of X-rays with matter and phase retrieval techniques. In the second section, 'Bacterial Samples', the three leading sample systems Deinococcus radiodurans, Bacillus subtilis and Bacillus thuringiensis are reviewed and concepts of the used sample preparation techniques both for $\mathrm{X}$-ray imaging and transmission electron microscopy are outlined. The third section, 'Experiments', gives an overview about the experimental achievements that are partly based on publications in $330,332,333$ (sections $3.1,3.3,3.4$ ), have been accepted for publication $[331]$ (section 3.2 ) or are in preparation for publication 329 (section 3.5).

- 'Ptychographic Imaging and Nano-Diffraction of D. radiodurans' outlines the concept of the combination of ptychographic imaging and scanning small angle X-ray scattering schemes. The former approach provides access to twoand three-dimensional maps of the electron density of the probed sample at a real-space resolution well below the beam size while the latter yields a scattering distribution with information corresponding to an even higher resolution in reciprocal space.

- 'Scanning Hard X-ray Microscopy Imaging Modalities for Geobiological Samples' is an a application of the combination of X-ray differential phase contrast and X-ray fluorescence on the geobiological sample of primary aragonitic elements of the demo-sponge Astrosclera willeyana and a quantitative phase contrast mapping on a consortium of sulfur-oxidizing bacteria with ptychography.

- 'STCS in High-Resolution X-Ray Ptychography Using KB Focusing' is dedicated to exploit the maximum available coherent light available at the GINIX for high-resolution, quantitative ptychographic imaging. The limitations set by count-rates of modern photon counting detectors were effectively overcome by using a Semi-Transparent Central Stop (STCS) which attenuates the primary X-ray beam in front of the detector to feasible levels.

- 'An STCS Application in High-Resolution X-Ray Ptychography' follows the line of the preceding section by presenting a high-flux ptychographic imaging experiment at the GINIX on solar cell nanowires as representatives of nonresolution-chart-type samples.

- 'Quantitative Waveguide-Based Imaging of B. thuringiensis and B. subtilis' exploits quantitative high-resolution X-ray phase contrast data on two bacterial systems of the genus Bacillus. The obtained electron density maps are used to determine the individual masses of the bacteria's enduring form of an endospore which allows for the bacteria's survival over long periods of nutritional starvation. 
Along the line of ptychographic imaging experiments, the sections also present different applications that are based on the availability of the reconstructed illuminating wave-field.

The thesis closes with a short summary, conclusion and an outlook. 



\section{Theory}

\subsection{Scalar Wave-Fields}

First, the basics of free-space propagation of scalar wave-fields will be addressed. Here, the differences between different imaging regimes are highlighted. Next, some numerical details of wave-field propagation using the discrete Fourier transform are discussed. Finally, basic results of the second order coherence theory are summarised yielding estimates for coherence lengths of specific synchrotron beamline set-ups.

\subsubsection{Propagation of Scalar Wave-Fields}

A rigorous treatment of the full theory of electromagnetic fields according to the Maxwell equations 190 can be found in textbooks such as $24,101,146,168,223$, 259 . Here, the traditional and practical treatment of X-ray wave-fields of $[101,223$ will be followed. The simplifications of

- assuming linear isotropic and static materials, which determines the relations between electric displacement and the electric field and magnetic induction and the magnetic field,

- the restriction to scalar fields, i.e. polarization effects are neglected,

- considering only non-magnetic materials, which is reflected by a constant magnetic permeability $\mu=\mu_{0}$, and

- the assumption that scatterers are slowly varying over length scales that are comparable to the $\mathrm{X}$-ray wavelength

are used. Taken together, these assumptions yield the following differential equation for scalar waves $\Psi(\vec{x}, t)$ :

$$
\left(\epsilon(\vec{x}) \mu_{0} \partial_{t}^{2}-\vec{\nabla}^{2}\right) \Psi(\vec{x}, t)=0
$$

where $\epsilon(\vec{x})$ denotes the electrical permittivity. The time dependence is separable by using the ansatz $\Psi(\vec{x}, t)=(2 \pi)^{-1 / 2} \int_{0}^{\infty} \psi_{\omega}(\vec{x}) \exp (-i \omega t) d \omega$. This yields the 'inhomogeneous' Helmholtz equation:

$$
\left(\vec{\nabla}^{2}+k^{2} n_{\omega}^{2}(\vec{x})\right) \psi_{\omega}(\vec{x})=0
$$

where $\omega=c k$ has been used $(k=2 \pi / \lambda$ and $c$ denote the wavenumber and the speed of light, respectively) and $n_{\omega}=c \sqrt{\epsilon_{\omega}(\vec{x}) \mu_{0}}$ is the frequency-dependent refractive index. The subscript indicates a dependence on the frequency $\omega$. Due to the high monocromaticity of the used synchrotron radiation of about $\Delta E / E \approx$ $10^{-4}$, the radiation can be considered to be quasi monochromatic. Henceforth, the 
subscript $\omega$ is omitted. The differential equation 1.2 is central for the theoretical description of X-rays in matter including the vacuum case where $n(\vec{x})=1$.

In the case of vacuum, elementary solutions to the 'homogeneous' Helmholtz equation 1.2 (with $n=1$ ) are given e.g. by plane waves $\propto \exp ( \pm i k \alpha)$ (with $\alpha=\{x, y, z\})$ and spherical waves $\propto \exp ( \pm i k r) / r\left(\right.$ with $\left.r=\sqrt{x^{2}+y^{2}+z^{2}}\right)$. Now consider the case that the electric disturbance $\psi(\vec{x})$ is known over a plane $z=z_{1}$ and the field over the plane $z=z_{1}+d$ is sought (cf. Fig. 1.2). Mathematically, this problem is stated as a boundary value problem that is solved by applying the integral theorem of Helmholtz and Kirchhoff or by following the RayleighSommerfeld formulation of diffraction. For instance, the first Rayleigh-Sommerfeld solution yields

$$
\psi\left(x^{\prime}, y^{\prime}, z_{1}+d\right)=\frac{k}{i 2 \pi} \iint_{\mathbb{R}^{2}} \psi\left(x, y, z_{1}\right) \frac{\exp (i k r)}{r} \frac{\vec{e}_{z} \cdot \vec{r}}{r} d x d y,
$$

where $\vec{e}_{z}$ denotes the unit vector in the z-direction. Note that this solution is effectively a weighted sum of the elementary spherical wave solutions and that only outgoing waves are considered.

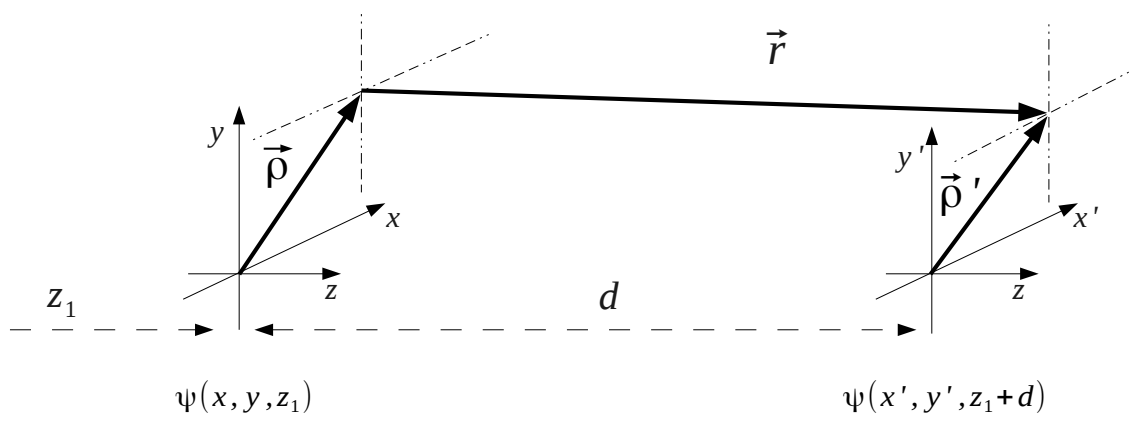

Figure 1.2: Sketch of the diffraction geometry between two planes $z=z_{1}$ and $z=z_{1}+d$. Adapted from |328].

Equation 1.3 can be further simplified by considering the case where $\left|x-x^{\prime}\right|, \mid y-$ $y^{\prime} \mid \ll d$, which is known as the Fresnel approximation. Consequently, the directional factor $\vec{e}_{z} \cdot \vec{r} / r$ can be neglected and the positional term $r=d \cdot \sqrt{1+\left(x-x^{\prime}\right)^{2} / d^{2}+\left(y-y^{\prime}\right)^{2} / d^{2}}$ can be approximated both in the exponential and its denominator. Usually, it is sufficient to use a zeroth order Taylor series for the denominator. The quickly oscillating exponential necessitates the first order Taylor series, i.e. $r / d \approx 1+\left(x-x^{\prime}\right)^{2} / 2 d^{2}+\left(y-y^{\prime}\right)^{2} / 2 d^{2}$, which yields

$$
\mathfrak{F}_{\mathfrak{R}}\left\{\psi\left(\vec{\rho}, z_{1}\right)\right\}\left(\overrightarrow{\rho^{\prime}}, d\right):=\frac{\exp (i k d)}{i d 2 \pi / k} \iint_{\mathbb{R}^{2}} \psi\left(\vec{\rho}, z_{1}\right) \exp \left(\frac{i k}{2 d}\left(\vec{\rho}-\overrightarrow{\rho^{\prime}}\right)^{2}\right) d \rho_{x} d \rho_{y}
$$


Here it should be noted that this result is essentially a convolution formulation of scalar wave-field diffraction (cf. 275 ). Equation 1.4 can also be written as

$$
\mathfrak{F}_{\mathfrak{R}}\left\{\psi\left(\vec{\rho}, z_{1}\right)\right\}\left(\overrightarrow{\rho^{\prime}}, d\right)=A\left(\overrightarrow{\rho^{\prime}}, d\right) \mathcal{F}\left\{A(\vec{\rho}, d) \psi\left(\vec{\rho}, z_{1}\right)\right\}\left(\overrightarrow{\rho^{\prime}} / \lambda d\right),
$$

where $A(\vec{\rho}, d)=\exp \left(i k \vec{\rho}^{2} / 2 d\right) \exp (i k d / 2) / \sqrt{i d 2 \pi / k}$ and the Fourier transform is written in terms of the spatial frequencies $\vec{\nu}=\overrightarrow{\rho^{\prime}} / \lambda d$. Notably, the weighting function $A(\vec{\rho}, d)$ can be highly oscillating. The Fourier transform $\mathcal{F}\{\cdot\}$ suggests a direct instruction for numerical evaluation of equation 1.4 by discretisation and use of the efficient 'Fast Fourier Transform' algorithm [49. The diffracted field in the plane $z=z_{1}+d$ can also be obtained by the 'angular spectrum' approach

$$
\mathfrak{F}_{\mathfrak{Q}}\left\{\psi\left(\vec{\rho}, z_{1}\right)\right\}\left(\overrightarrow{\rho^{\prime}}, d\right):=\mathcal{F}^{-1}\left\{\exp \left(i d \sqrt{k^{2}-k_{x}^{2}-k_{x}^{2}}\right) \mathcal{F}\left\{\psi\left(\vec{\rho}, z_{1}\right)\right\}\right\}
$$

where $\vec{k}=2 \pi \vec{\nu}$. Firstly, it should be noted that equation 1.6 becomes identical to the Rayleigh-Sommerfeld convolution formulation of equation 1.4 if the argument of the exponential is approximated by using $\sqrt{k^{2}-k_{x}^{2}-k_{y}^{2}} \approx k-\left(k_{x}^{2}+k_{y}^{2}\right) / 2 k$. Secondly, the mathematical formulation of equation 1.6 reveals that diffraction can be understood as a filtering operation on the field $\psi\left(\vec{\rho}, z_{1}\right)$. However, the filtering is of complex nature. The filtering kernel $\exp \left(i d \sqrt{k^{2}-k_{x}^{2}-k_{y}^{2}}\right)$ is a highly oscillating and complex function that also cancels out certain frequency components of the diffracting field.

Before discussing practical issues of numerical simulation of the propagation of scalar wave-fields, the limiting case of the Fraunhofer regime is considered next. If the observation plane $z=z_{1}+d$ is sufficiently far away in comparison to the lateral extent of the source disturbance in the plane $z=z_{1}$ the quadratic phase term $\exp \left(i k \vec{\rho}^{2} / 2 d\right)$ (also called 'chirp' function) of $A(\vec{\rho}, d)$ in equation 1.4 can be omitted. The traditional measure of 'far away' is the Fresnel number [168 259

$$
F=k w^{2} / 2 \pi d,
$$

where $w$ denotes the maximum radial extent of an object such as a circular aperture. If $F \ll 1$ equations $1.4,1.5$ simplify to

$$
\mathfrak{F}_{\mathfrak{R}}\left\{\psi\left(\vec{\rho}, z_{1}\right)\right\}\left(\overrightarrow{\rho^{\prime}}, d\right) \approx A^{\prime}\left(\overrightarrow{\rho^{\prime}}, d\right) \mathcal{F}\left\{\psi\left(\vec{\rho}, z_{1}\right)\right\}\left(\overrightarrow{\rho^{\prime}} / \lambda d\right),
$$

where $A^{\prime}\left(\overrightarrow{\rho^{\prime}}, d\right)=\exp \left(i k{\overrightarrow{\rho^{\prime}}}^{2} / d\right) \exp (i k d) /(i d / k)$. On the other hand, large Fresnel numbers $F \geq 1$ and $F \gg 1$ denote the Fresnel and contact regime, respectively. 
An illustration of the different imaging regimes is presented in figure 1.3 . For those examples, diffracted intensities of the following two objects

$$
\begin{aligned}
& \psi_{a}\left(\vec{\rho}, z_{1}\right)=\chi_{*}(\vec{\rho}) \quad \text { and } \\
& \psi_{p}\left(\vec{\rho}, z_{1}\right)=\exp \left(i \cdot 0.1 \cdot \chi_{*}(\vec{\rho})\right)
\end{aligned}
$$

are calculated, where $\chi_{*}(\vec{\rho})$ denotes the characteristic function of a Siemens star with 16 segments. The objects of equations 1.10 and 1.9 are shown in rows $1,3,5$ and rows 2,4,6 of figure 1.3. Although, the Fresnel number yields a good measure of the validity of the Fraunhofer approximation it is often useful to compare it with the range of validity of the Fresnel diffraction formulas 1.4, 1.6. For this reason, the reader is referred to the article by Rees 246 . 


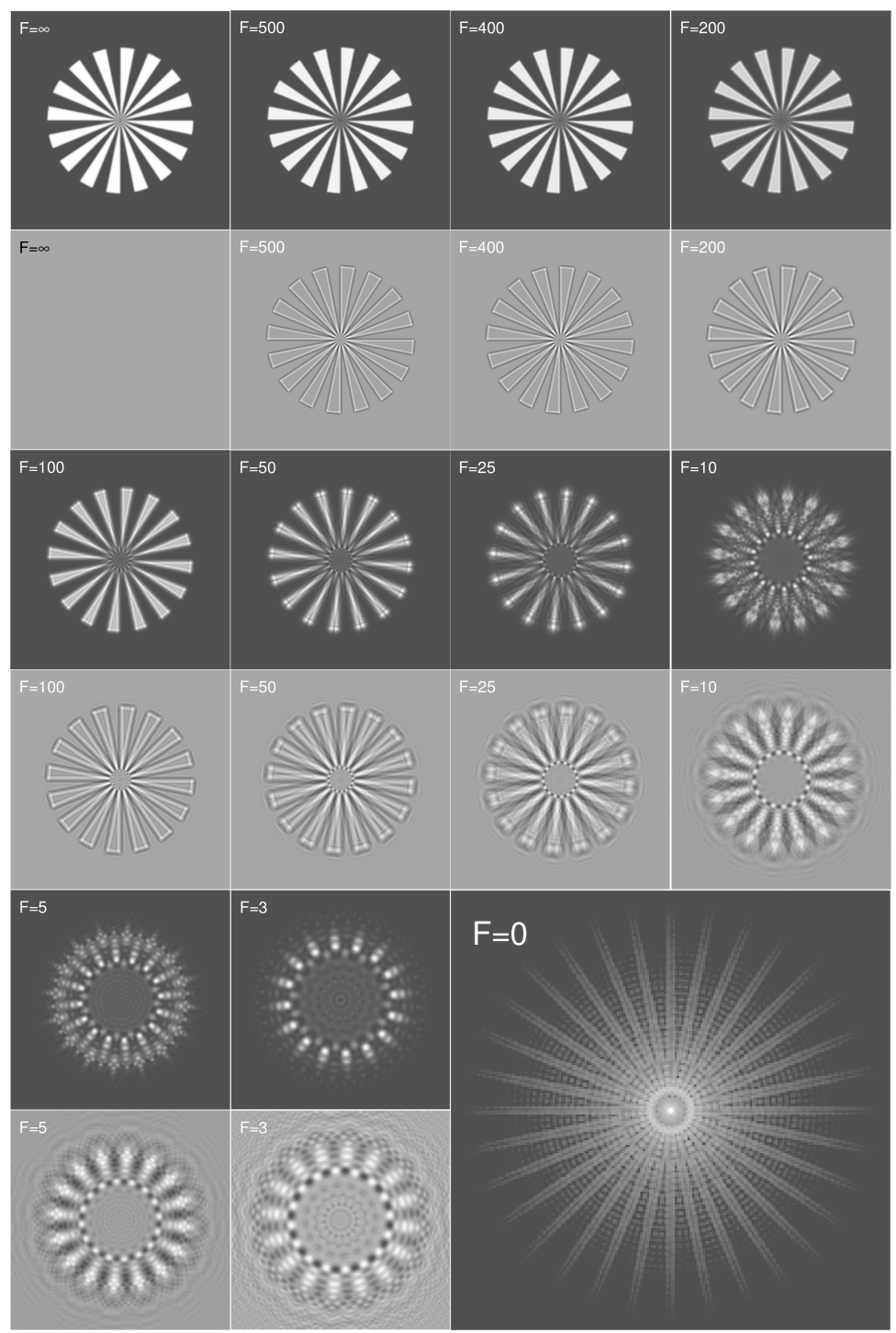

Figure 1.3: This figure illustrates diffraction of the Siemens star objects $\psi_{a}$ (rows 1,3,5) and $\psi_{p}$ (rows 2,4,6) due to propagation as a function of the Fresnel number. In this example, the Fresnel numbers $\infty \geq F \geq 100,100 \geq F>1$ and $1 \gg F \geq 0$ correspond approximately to the contact, Fresnel and Fraunhofer regime, respectively. Note that: (i) in the contact regime phase contrast evolves by edge enhancement (2nd row) and the amplitude object appears to change more slowly, (ii) in the Fresnel region both objects quickly loose the resemblance to their original form and the images become holographic, (iii) in the farfield limit $F=0$ only the diffraction pattern of the pure amplitude object is shown in logarithmic scaling (6 orders of magnitude) and (iv) $\mathfrak{F}_{\mathfrak{Q}}\{\cdot\}$ and $\mathfrak{F}_{\mathfrak{R}}\{\cdot\}$ were used for $F=500, \ldots, 3$ and $F=0$, respectively. This figure is inspired by presentations in $6,12,90,267]$. 


\subsubsection{Remarks on the Numerical Propagation of Scalar Wave-Fields}

In case of numerical evaluation of e.g. equations 1.4 and 1.6 , caution is advised as this unavoidably yields discretisation and truncation effects that need to be understood in order to minimise artefacts. The 'Whittaker-Shannon Sampling Theorem' 273, 327 makes a very important theoretical statement about discretisation. Here, its presentation in 101 will be briefly outlined.

Consider the case of a band-limited function $g(x, y)$ whose Fourier transform is non zero only over a finite region $\mathcal{R}: \mathcal{F}\{g\}\left(\nu_{x}, \nu_{y}\right)=0 \forall \nu_{x}, \nu_{y} \notin \mathcal{R}$. Let $V_{x}, V_{y}$ be the maximum moduli of the frequencies within $\mathcal{R}$. A fine sampling in real space

$$
\Delta_{i} \leq \frac{1}{2 V_{i}}, i=x, y
$$

assures successful recovery of the continuous function $g(x, y)$ by its samples $g_{n, m}=$ $g\left(n \Delta_{x}, m \Delta_{y}\right)$

$$
g(x, y)=\sum_{n, m=-\infty}^{\infty} g_{n, m} \frac{\sin \left(\left(x-n \Delta_{x}\right) / \Delta_{x}\right)}{\left(x-n \Delta_{x}\right) / \Delta_{x}} \frac{\sin \left(\left(y-m \Delta_{y}\right) / \Delta_{y}\right)}{\left(y-m \Delta_{y}\right) / \Delta_{y}}
$$

It should be noted that in the derivation of equation 1.12 the specific choice of a rectangular filter is used which determines the interpolation function for values between the samples $g_{n, m}$ to be a $\operatorname{sinc}(\cdot)$ function (cf. [101]). Importantly, for the case of a band-limited continuous function $g(x, y)$ the Whittaker-Shannon sampling theorem states that it can be fully described by its samples $g_{n, m}$. In practice, the infinite series in equation 1.12 may also be approximated by a finite number of summands.

Calculation of the Fourier transformation for reasons of propagation of scalar wave-fields according to equations 1.4 and 1.6 is carried out by approximation of the integral by a finite sum by using the trapezoidal ${ }^{1}$ rule 25,238 :

$$
\begin{aligned}
\mathcal{F}\{f(x)\}\left(\nu_{n}\right) & =\int_{-\infty}^{\infty} f(x) e^{-2 \pi i \nu_{n} x} d x \approx \sum_{k=0}^{N-1} f_{k} e^{-2 \pi i \nu_{n} x_{k}} \Delta x \\
& =\Delta x \sum_{k=0}^{N-1} f_{k} e^{-2 \pi i k n / N}=N \Delta x \operatorname{DFT}\left\{f_{k}\right\}(n),
\end{aligned}
$$

where a sampling $x_{k}=k \Delta x(k=0, \ldots, N-1)$ and $\nu_{n}=n / N \Delta x(n=-N / 2, \ldots, N / 2)$ was assumed in real and reciprocal space, respectively. In addition, in the last step the definition of the Discrete Fourier Transform DFT $\{\cdot\}$ has been introduced. The used sampling of $\Delta \nu \Delta x=1 / N$ yields

$$
\Delta x^{\prime} \Delta x=\frac{\lambda d}{N}
$$

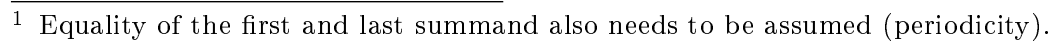


where the physical dimensions of equation 1.5 relate the physical dimensions in the planes $z=z_{1}(\Delta x)$ and $z=z_{1}+d\left(\Delta x^{\prime}\right)$ to each other. As a consequence of the different sampling lengths, the covered area of the source plane differs from that in the destination plane. On the contrary, the propagation of scalar wave-fields according to equation 1.6 yields the same sampling in both planes. In the context of the DFT the highest frequency

$$
\nu_{N y}=\frac{1}{2 \Delta x}
$$

is known as 'Nyquist' or 'critical' frequency. In accordance to the WhittakerShannon sampling theorem, it is assumed that a sampling rate at the reciprocal of twice the Nyquist frequency yields an accurate spectral representation. Insufficient sampling yields 'aliasing' artefacts. In this case, the spectral contributions above the Nyquist frequency appear to be falsely reflected into the accessible frequency range below $\nu_{N y}$. It is thus often better to sample higher than at Nyquist rate. For instance, Voelz and Roggemann 317 recommend to sample the chirp function of the plane $z=z_{1}$ of equation 1.5 at

$$
\Delta x \leq \frac{\lambda d}{L}
$$

where $L$ denotes the full sampling window. In this case, the phase of the chirp function does not change more than $\pi$ between two neighbouring samples. In principal, the sampling of the outer chirp function $A\left(\overrightarrow{\rho^{\prime}}, d\right)$ also needs to be adapted but often this factor can be neglected. Similarly, the sampling requirement for Fresnel approximation of the chirp function of equation 1.6 is obtained:

$$
\Delta x \geq \frac{\lambda d}{L} .
$$

Taken together, these two sampling conditions reflect the different regimes of validity of the two approaches for wave-field propagation. Care needs also to be taken when choosing the sampling range (window) as the DFT is primarily adequate for periodic signals. However, in practice, the data is rarely periodic. For this reason, it is recommended to use (i) a window function and (ii) zero-padding $[32]$.

In particular, the use of a window function is of importance for calculating the the power spectral density (PSD) in the context of this work (cf. sections 3.1, p. 69 and 3.3 p. 132 ). For instance, a window function $w(x)$ results in a convolution operation in the Fourier domain (cf. equation 1.142, p. 191):

$$
\mathcal{F}\{f \cdot w\}=\mathcal{F}\{f\} \star \mathcal{F}\{w\} .
$$

Intrinsically, the DFT calculation implies a convolution with a sinc(·) function due to the finite length of the data. The benefit of special window functions is illustrated by calculating the power spectral density of the function

$$
\begin{aligned}
f(x)= & \cos (2 \pi x)+10^{-2} \cos (1.15 \cdot 2 \pi x)+10^{-3} \cos (1.25 \cdot 2 \pi x)+ \\
& 10^{-3} \cos (2 \cdot 2 \pi x)+10^{-4} \cos (2.75 \cdot 2 \pi x)+10^{-5} \cos (3 \cdot 2 \pi x)(1.20)
\end{aligned}
$$


without and with the use of the 'Kaiser-Bessel' window

$$
K_{\beta}(x)=\left\{\begin{array}{lll}
J_{0}\left(\beta \sqrt{1-(2 x / L)^{2}}\right) / J_{0}(\beta) & \text { for } \quad-L / 2 \leq x \leq L / 2 \\
0 & \text { else }
\end{array}\right.
$$

as exemplified in $\mid 32$. The use of the Kaiser-Bessel window may facilitate the visibility of weak frequency contributions to the power spectral density. In figure 1.4 the use of the window function allows to observe all the frequencies $\nu>1$ whereas these frequencies are not apparent otherwise. The disadvantage of using special window functions is that they result in a loss in frequency resolution. That is, peaks in the spectrum become broadened. Consequently, it may be impossible to distinguish two peaks occurring very close to each other. Nevertheless, the Kaiser-Bessel window has been proven quite useful for determination of the power spectral densities of X-ray phase contrast images of e.g. biological cells in order to estimate their frequency content $[95]$.
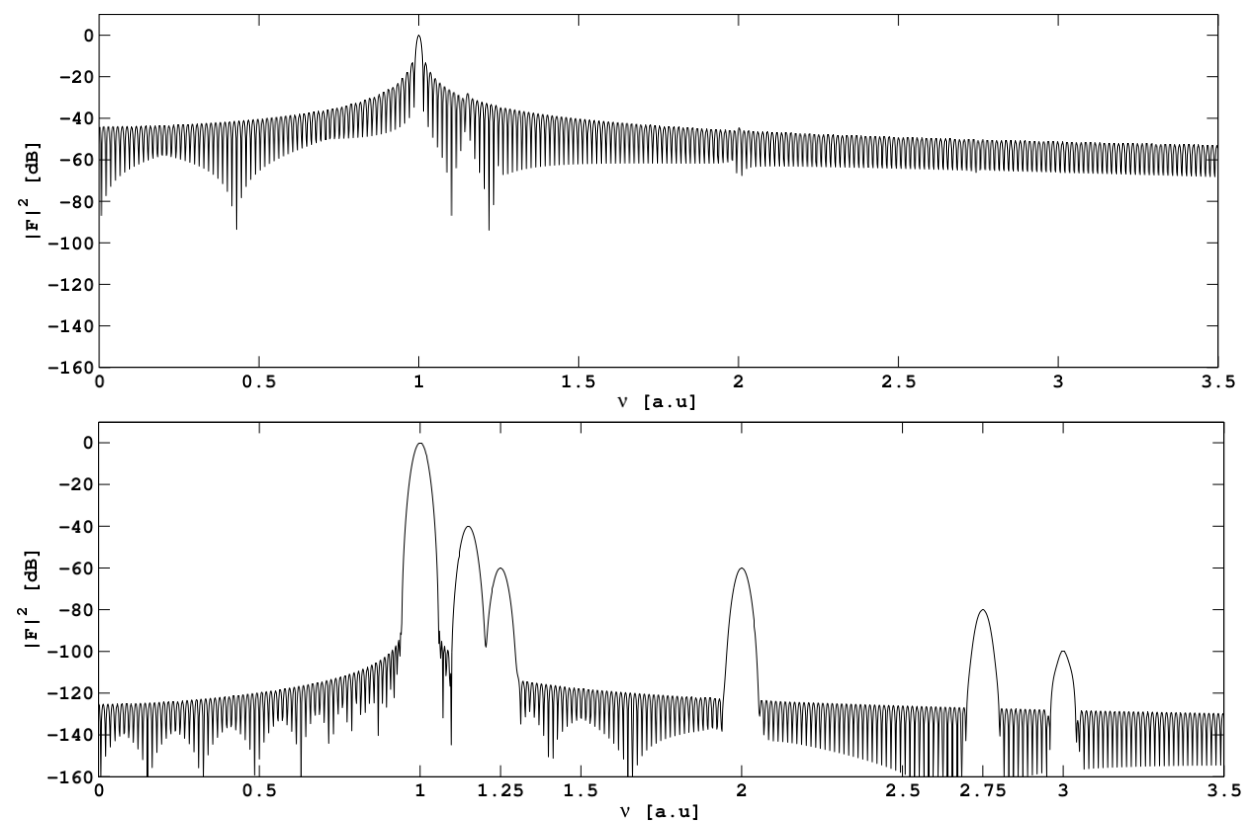

Figure 1.4: The figure illustrates the advantage of using window functions such as the Kaiser-Bessel window. The upper graph shows the power spectral density (decibel: $\left.\mathrm{dB}=10 \log _{10}(x)\right)$ as calculated of the function $f(x)$ of equation 1.20 The lower graph presents the result for the case of using the Kaiser-Bessel window $(\beta=12)$ where also all frequency contributions $>1$ can be clearly identified. Both graphs have been calculated with a sampling rate of $5 \nu_{N y}$ using $2^{10}$ samples. In addition the data have been padded with zeros to a final array length of $2^{14}$. This example is adapted from [32]. 


\subsubsection{Partial Coherence}

An essential underlying physical effect that gives rise to many of the results of this work is known as interference. The consequence of partial coherence often appearing as a smearing of the diffraction data is unwanted as it may prevent successful phase retrieval. However, in order to create interference contrast the partaking wave-fields do not necessarily need to be fully coherent and a small deviation from full coherence is often tolerable. Here, a few basic concepts of the so-called 'second-order coherence theory' will be listed. A textbook treatment of the theory of partial coherent wave-fields can be found in the works [186, 337]. The reader who is interested in phase-retrieval with an emphasis also on partial coherence is referred to the following review articles $216,243$.

Firstly, we note that light is never purely monochromatic. A good approximation also with respect to monochromatised synchrotron radiation $\left(\Delta E / E \approx 10^{-4}\right)$ is the assumption of 'quasi-monochromatic' light, i.e. there is a small bandwidth associated with the mean frequency $\bar{\omega}$

$$
\frac{\Delta \omega}{\bar{\omega}} \ll 1 .
$$

In addition, it is important to be fully aware of the fact that the measurable intensities correspond to a time average

$$
\langle I\rangle_{t}:=\lim _{T \rightarrow \infty} \frac{1}{2 T} \int_{-T}^{T} I(t) d t .
$$

An essential quantity of the second order coherence theory is the so called 'mutual coherence function'

$$
\Gamma\left(\vec{r}_{1}, \vec{r}_{2}, \tau\right):=\left\langle\psi^{*}\left(\vec{r}_{1}, t\right), \psi\left(\vec{r}_{2}, t+\tau\right)\right\rangle_{t}
$$

which measures the correlation of the product of the field variable $\psi$ at two points in space. Setting $\vec{r}_{1}=\vec{r}_{2}=\vec{r}$ yields the intensity at the point $\vec{r}$. The normalised form

$$
\gamma\left(\vec{r}_{1}, \vec{r}_{2}, \tau\right):=\frac{\Gamma\left(\vec{r}_{1}, \vec{r}_{2}, \tau\right)}{\sqrt{\Gamma\left(\vec{r}_{1}, \vec{r}_{1}, 0\right)} \sqrt{\Gamma\left(\vec{r}_{2}, \vec{r}_{2}, 0\right)}}=\frac{\Gamma\left(\vec{r}_{1}, \vec{r}_{2}, \tau\right)}{\sqrt{I\left(\vec{r}_{1}\right)} \sqrt{I\left(\vec{r}_{2}\right)}}
$$

is known as 'complex degree of coherence'.

It can be shown that

$$
0 \leq\left|\gamma\left(\vec{r}_{1}, \vec{r}_{2}, \tau\right)\right| \leq 1
$$

holds. At the extreme cases of $\left|\gamma\left(\vec{r}_{1}, \vec{r}_{2}, \tau\right)\right|=0$ and $\left|\gamma\left(\vec{r}_{1}, \vec{r}_{2}, \tau\right)\right|=1$ the disturbances at $\vec{r}_{1}$ and $\vec{r}_{2}$ are considered to be 'mutually completely incoherent' and 'mutually completely coherent', respectively. Using these quantities one finds the intensity at a point $P$ on a screen due to the interference from two disturbances at the pinholes $Q_{1}, Q_{2}$ 303,337 (cf. Fig. 1.5)

$$
I(P)=I_{0}(P)\left(1+\left|\gamma\left(Q_{1}, Q_{2}, \tau\right)\right| \cos \left(\alpha\left(Q_{1}, Q_{2}, \tau\right)-\delta\right)\right) .
$$


Here, the intensity due to the pinholes is assumed to be the same, that is $I_{0}(P)$. $\delta$ is the phase difference due to the different path lengths $\left|\vec{r}_{1}\right|,\left|\vec{r}_{2}\right|$ or equivalently the relative time shift $\tau=t_{1}-t_{2}$ between disturbances

$$
\delta=\bar{\omega} \tau=\bar{\omega}\left(t_{2}-t_{1}\right)=\frac{2 \pi}{\bar{\lambda}}\left(\left|\vec{r}_{2}\right|-\left|\vec{r}_{1}\right|\right)
$$

The phase envelope $\alpha\left(Q_{1}, Q_{2}, \tau\right)=\bar{\omega} \tau+\operatorname{Arg}\left(\gamma\left(Q_{1}, Q_{2}, \tau\right)\right)$ is expected to change slowly in comparison to the time interval $\tau \ll 1 / \Delta \omega$. Evidently, the contrast of
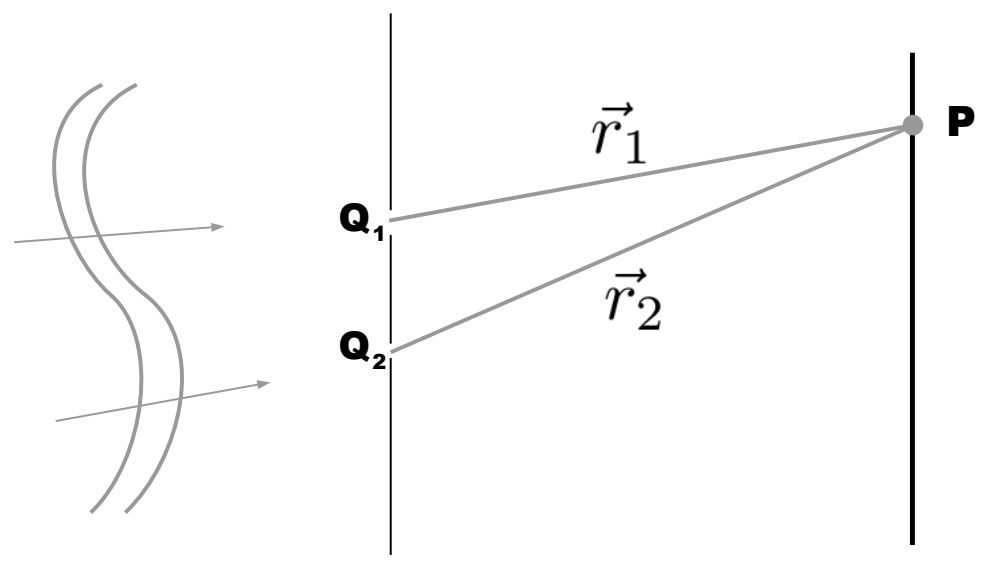

Figure 1.5: The figure sketches the experimental geometry for the Young's interference pattern as discussed in the text for the case of partially coherent quasi-monochromatic light. Graphic adapted from [337].

the cosine oscillation in equation 1.27 depends on the modulus of the complex degree of coherence. Using the definition of the 'visibility'

$$
\mathcal{V}:=\frac{I_{\max }(P)-I_{\min }(P)}{I_{\max }(P)+I_{\min }(P)},
$$

one finds for the Young's interference pattern due to partially coherent quasimonochromatic light:

$$
\mathcal{V}=\left|\gamma\left(Q_{1}, Q_{2}, \tau\right)\right|
$$

Importantly, the visibility relates a measurable quantity to the complex degree of coherence. Maximum visibility $\mathcal{V}=1$ thus corresponds to mutual complete coherence whereas minimum visibility $\mathcal{V}=0$ corresponds to mutual complete incoherence. The $6+1$ dimensional field $\gamma\left(Q_{1}, Q_{2}, \tau\right)$ is well suited to describe the effects of both temporal coherence (Michelson interferometer) and spatial coherence (Young's interference experiment). If one considers spatial coherence, it is 
convenient to choose a symmetric set-up where $\tau=0$. For this reason one defines the 'mutual intensity'

$$
J\left(\vec{r}_{1}, \vec{r}_{2}\right):=\Gamma\left(\vec{r}_{1}, \vec{r}_{2}, 0\right)=\left\langle\psi^{*}\left(\vec{r}_{1}, t\right) \psi\left(\vec{r}_{2}, t\right)\right\rangle_{t}
$$

and the 'equal time complex degree of coherence'

$$
\begin{aligned}
j\left(\vec{r}_{1}, \vec{r}_{2}\right) & :=\gamma\left(\vec{r}_{1}, \vec{r}_{2}, 0\right) \\
& =\frac{J\left(\vec{r}_{1}, \vec{r}_{2}\right)}{\sqrt{J\left(\vec{r}_{1}, \vec{r}_{1}\right)} \sqrt{J\left(\vec{r}_{2}, \vec{r}_{2}\right)}}=\frac{J\left(\vec{r}_{1}, \vec{r}_{2}\right)}{\sqrt{I\left(\vec{r}_{1}\right)} \sqrt{I\left(\vec{r}_{2}\right)}} .
\end{aligned}
$$

In case of quasi-monochromatic light one can approximate the mutual coherence function and the complex degree of coherence

$$
\begin{aligned}
& \Gamma\left(\vec{r}_{1}, \vec{r}_{2}, \tau\right) \approx J\left(\vec{r}_{1}, \vec{r}_{2}\right) \exp (-i \bar{\omega} \tau), \\
& \gamma\left(\vec{r}_{1}, \vec{r}_{2}, \tau\right) \approx j\left(\vec{r}_{1}, \vec{r}_{2}\right) \exp (-i \bar{\omega} \tau),
\end{aligned}
$$

where it is assumed that $|\tau| \ll 2 \pi / \Delta \omega$, i.e. the time shift is much smaller than the coherence time. An important insight with respect to spatial coherence is obtained from the 'Van Cittert-Zernike theorem' [341] which relates a spatially incoherent source to the equal time complex degree of coherence at two arbitrary points $\vec{r}_{1}$, $\vec{r}_{2}$ in a distance $d$ away from the source. Assuming quasi-monochromatic light and that the source is statistically stationary (in the wide sense) one finds [337. (cf. Fig. 1.6):

$$
j\left(\vec{r}_{1}, \vec{r}_{2}\right)=\frac{1}{\sqrt{I\left(\vec{r}_{1}\right)} \sqrt{I\left(\vec{r}_{2}\right)}} \int_{\sigma} I(\rho) \frac{\exp \left(i \bar{k}\left(\left|\vec{r}_{2}\right|-\left|\vec{r}_{1}\right|\right)\right)}{\left|\vec{r}_{1}\right|\left|\vec{r}_{2}\right|} d x d y
$$

where $I\left(\vec{r}_{i}\right)=J\left(\vec{r}_{i}, \vec{r}_{i}\right)=\int_{\sigma} \frac{I(\rho)}{\left|\vec{r}_{i}\right|^{2}} d x d y(i=1,2)$ and $I(\vec{\rho})$ is the mean intensity distribution of the source. Using the approximation $\left|\vec{r}_{j}\right| \approx d+\left(\vec{\rho}-\vec{\rho}^{\prime}{ }_{j}\right)^{2} / 2 d$ in the exponential of equation 1.35 and replacing $\left|\vec{r}_{j}\right|$ in the denominators by $\left|\vec{r}_{j}\right| \approx d$, one finds the 'far-zone' form of the van Cittert-Zernike theorem

$$
j\left(\vec{r}_{1}, \vec{r}_{2}\right)=e^{i \frac{{\overrightarrow{\rho^{\prime}}}_{2}^{2}-{\overrightarrow{\rho^{\prime}}}_{1}^{2}}{\bar{\lambda} d}} \cdot \frac{\iint_{\sigma} I(\vec{\rho}) \exp (-i 2 \pi \vec{\delta} \cdot \vec{\rho} /(\bar{\lambda} d)) d \rho_{x} d \rho_{y}}{\iint_{\sigma} I(\vec{\rho}) d \rho_{x} d \rho_{y}} .
$$

In the far-zone the equal time complex degree of coherence of an incoherent, extended source thus depends on the Fourier transform of the intensity distribution in the source plane and a phase factor

$$
j\left(\vec{r}_{1}, \vec{r}_{2}\right)=e^{i \Psi} \cdot \frac{\mathcal{F}\{I\}(\vec{\delta} / \bar{\lambda} d)}{\mathcal{F}\{I\}(0)}
$$




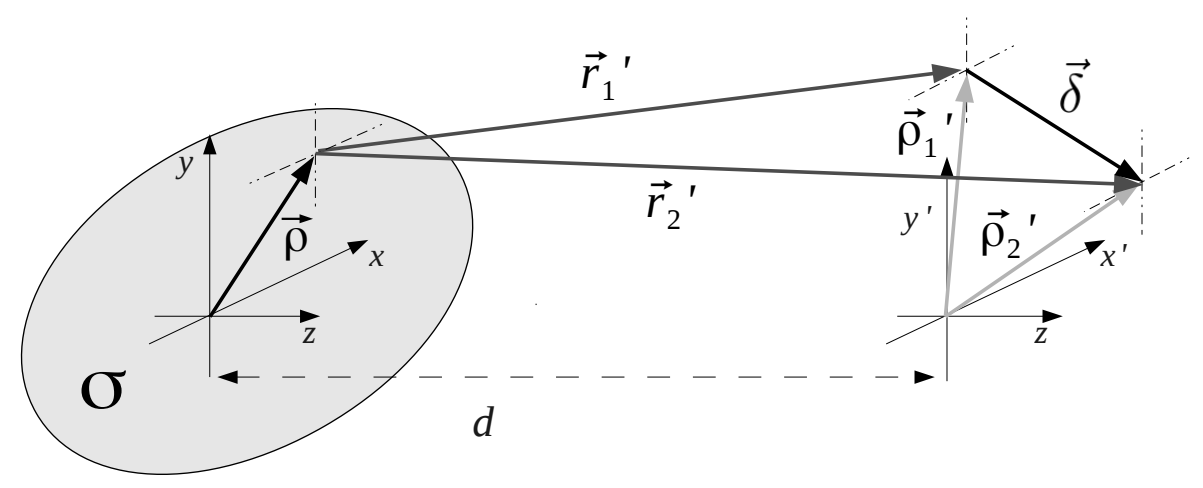

Figure 1.6: This figure illustrates the geometry of the Van Cittert-Zernike theorem. The equal time degree of complex coherence $j\left(\vec{r}_{1}, \vec{r}_{2}\right)$ depends solely on the geometry and the intensity distribution of the incoherent source $\sigma$.

where $\Psi=\pi\left({\overrightarrow{\rho^{\prime}}}_{2}^{2}-{\overrightarrow{\rho^{\prime}}}_{1}^{2}\right) / \bar{\lambda} d$.

A synchrotron undulator source can be well described by a Gaussian intensity distribution that is separable in the $\mathrm{x}$ - and $\mathrm{y}$ - directions

$$
\begin{aligned}
I(\vec{\rho}) & =I_{0} I^{G}\left(\rho_{x}\right) I^{G}\left(\rho_{y}\right), \text { where } \\
I^{G}\left(\rho_{j}\right) & =\frac{1}{\sqrt{2 \pi} \sigma_{j}} \exp \left(-\frac{1}{2} \frac{\rho_{j}^{2}}{\sigma_{j}^{2}}\right), \quad j=x, y .
\end{aligned}
$$

Note that, the relation between $\sigma$ and the Full-width at half maximum (FWHM) is given by

$$
\mathrm{FWHM}=2 \sqrt{2 \log (2)} \sigma \approx 2.355 \sigma .
$$

One finds for the modulus of the equal time complex degree of coherence

$$
|j(\vec{\delta})|=\exp \left(-\frac{1}{2} \frac{\delta_{x}^{2}}{\left(\bar{\lambda} d / 2 \pi \sigma_{x}\right)^{2}}\right) \exp \left(-\frac{1}{2} \frac{\delta_{y}^{2}}{\left(\bar{\lambda} d / 2 \pi \sigma_{y}\right)^{2}}\right) \equiv j\left(\delta_{x}\right) j\left(\delta_{y}\right) .
$$

Here, we consider the case that the directional equal time complex degree of coherence $j\left(\delta_{x, y}\right)$ drops to the value $1 / e$ (corresponding to $\mathcal{V}=1 / e$ ) in order to estimate the coherence length $\delta_{x, y}^{1 / e}$ at a distance $d$ from the undulator source:

$$
\Rightarrow \delta_{x, y}^{1 / e}=\frac{1}{\sqrt{2} \pi} \frac{\bar{\lambda} d}{\sigma_{x, y}} .
$$

Hence, the (spatial) coherence length $\delta_{x, y}^{1 / e}$ increases with distance to the source and decreasing source size. The light from an extended incoherent source thus becomes (spatially) coherent by free-space propagation. However, the coherence length 
decreases with increasing energy. Some typical $\delta^{1 / e}$ values for specific beamlines are given in tables 1.1 and 1.2 .

In view of the experiments within this thesis, the spatial coherence properties are primarily relevant but it may also be helpful to have an estimation for the temporal coherence length $\delta_{t}$. Considering two interfering waves with wavelength $\lambda$ and $\lambda-\Delta \lambda$, one deduces a temporal coherence length of $[6$

$$
\delta_{t} \approx \frac{1}{2} \frac{\lambda^{2}}{\Delta \lambda} .
$$

Using the relation $E=h \nu=h c / \lambda$ ( $h$ Planck's constant and $c$ speed of light), it follows that $\Delta E=E \Delta \lambda / \lambda$. Hence, $\delta_{t}=\frac{1}{2} \frac{E}{\Delta E} \lambda$. For the case of $\Delta E / E \approx 10^{-4}$ (an undulator in combination with e.g. a double crystal monochromator $\mathrm{Si}(111)$ ) and a typical X-ray wavelength within this work of $2 \AA$, this yields $\delta_{t}=1 \mu \mathrm{m}$. Note that for quasi monochromatic light, it follows that the coherence length is given by 337

$$
\delta_{t} \approx \frac{2 \pi c}{\Delta \omega}=\frac{\lambda^{2}}{\Delta \lambda}
$$

which differs by a factor of 2 .

Table 1.1: The table lists the calculated coherence lengths in round figures for different slit positions S0, S1, S3 of the cSAXS beamline at the Swiss Light Source according to equation 1.42 corresponding to a $1 / e$ visibility. The source size at the cSAXS beamline is given approximately by $\sigma_{v}=8 \mu \mathrm{m}$ and $\sigma_{h}=86 \mu \mathrm{m}$ using typical values at $12.4 \mathrm{keV}$ (cf. http://www.psi.ch/sls/csaxs/source).

\begin{tabular}{ccccc}
\hline & & S0 & S1 & S2 \\
$E[\mathrm{keV}]$ & $\mathrm{d}[\mathrm{m}]$ & 12 & 26 & 30.7 \\
\hline \multirow{2}{*}{6.2} & $\begin{array}{c}\delta_{h}^{1 / e}[\mu \mathrm{m}] \\
\delta_{v}^{1 / e}[\mu \mathrm{m}]\end{array}$ & $\begin{array}{cccc}1 \\
61\end{array}$ & 153 & 181
\end{tabular}


Table 1.2: The table lists the calculated coherence lengths in round figures for one slit positions G1 (distance to source $84 \mathrm{~m}$ ) of the P10 beamline at PETRA III according to equation 1.42 corresponding to a $1 / e$ visibility. The source size at the P10 beamline is given approximately by $\sigma_{v}=5.6 \mu \mathrm{m}, \sigma_{h}=140 \mu \mathrm{m}$ (high beta) and $\sigma_{v}=6.1 \mu \mathrm{m}, \sigma_{h}=36 \mu \mathrm{m}$ (low beta) using typical values at $10 \mathrm{keV}$ (cf. http://photon-science.desy.de/facilities/petra_iii/beamlines/ p10_coherence_applications/beamline_specifications/index_eng.html). Experiments within this thesis were carried out with a low-beta source.

\begin{tabular}{cccc}
\hline & $E[\mathrm{keV}]$ & 7.9 & 13.8 \\
\hline \multirow{3}{*}{ low beta } & $\delta_{h}^{1 / e}[\mu \mathrm{m}]$ & 82 & 47 \\
& $\delta_{v}^{1 / e}[\mu \mathrm{m}]$ & 486 & 278 \\
& & & \\
high beta & $\delta_{h}^{1 / e}[\mu \mathrm{m}]$ & 21 & 12 \\
& $\delta_{v}^{1 / e}[\mu \mathrm{m}]$ & 530 & 303 \\
& & & \\
\hline
\end{tabular}

\subsection{X-Rays in Matter}

The discussion about the implications of X-rays interacting with matter is started from an atomistic scattering approach. Following $[6,124,161,255$ the scattering approach allows to make a transition from atomic scales to the macroscopically relevant quantities of the refractive index that is determined by material constants. These constants were measured by Henke and co-workers 125 allowing precise predictions of experimental expectations ${ }^{2}$. Thereafter, the derivation of the projection approximation, which describes the imprint of an illuminated object onto the incident $\mathrm{X}$-ray wave-field in terms of the complex index of refraction, will be outlined according to 223 . Finally, the important relation between invested dose and experimental achievable resolution is discussed.

\subsubsection{Scattering Theory}

The scattering strength of a probed object is determined by the scattering crosssection $\sigma$, which is obtained by integrating the differential cross-section of a given experiment. Following the theoretical description by Als-Nielsen and McMorrow [6, the Thomson differential scattering cross-section of an electromagnetic wave by a free electron is given as (cf. Fig. 1.7)

$$
\left(\frac{d \sigma}{d \Omega}\right)=\frac{I_{\mathrm{sc}}}{\Phi_{0} \Delta \Omega}=r_{0}^{2} P,
$$

where $\Delta \Omega, I_{\mathrm{sc}}, \Phi_{0}, r_{0}=e^{2} / 4 \pi \epsilon_{0} m c^{2}=2.82 \mathrm{fm}$ and $P$ denote the solid angle of the scattering radiation, the scattering intensity, the flux of the incident beam, the

\footnotetext{
2 A practical access to the data is available on the internet: http://www.cxro.lbl.gov/
} 
classical radius of the electron (or Thomson scattering length) and the polarisation factor of the incoming and outgoing wave, namely

$$
P=\left\{\begin{array}{cl}
1 & \text { synchrotron: vertical scattering plane } \\
\cos ^{2} \Psi & \text { synchrotron: horizontal scattering plane } \\
\frac{1}{2}\left(1+\cos ^{2} \Psi\right) & \text { unpolarised source }
\end{array}\right.
$$

respectively. Here, the angle $\Psi+\pi$ denotes the angle in the horizontal plane between the polarisation vectors of the incoming and outgoing radiation (cf. Fig. 1.6 in $[6]$ ). Notably, the last equation indicates that the scattering signal is favourably detected along the vertical direction in a synchrotron scattering experiment.

Next, the classical description of scattering by an electron distribution of an atom that is determined by the number density $\rho(\vec{r})$ is treated. One finds for the total scattering length of an atom:

$$
-r_{0} f^{0}(\vec{Q})=-r_{0} \int_{V \in \mathbb{R}^{3}} \rho(\vec{r}) \exp (i \vec{Q} \cdot \vec{r}) d \vec{r}
$$

where the geometry for the scattering vector $\vec{Q}=\vec{k}-\overrightarrow{k^{\prime}}$ is depicted in figure $1.7(\vec{k}$ and $\overrightarrow{k^{\prime}}$ denote incoming and outgoing wave vector, respectively). In the limiting case of high and low wave vector transfer the scattering amplitude yields

$$
\begin{aligned}
& \lim _{|\vec{Q}| \rightarrow 0} f^{0}(\vec{Q})=Z, \text { and } \\
& \lim _{|\vec{Q}| \rightarrow \infty} f^{0}(\vec{Q})=0 .
\end{aligned}
$$

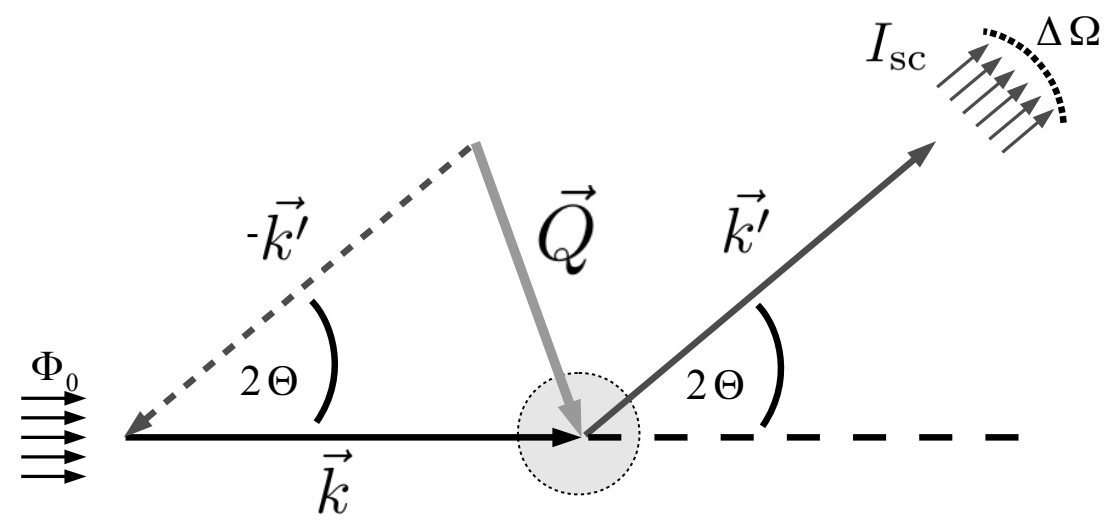

Figure 1.7: The figure sketches the typical scattering geometry: an incoming wave $\vec{k}$ is scattered into a wave $\overrightarrow{k^{\prime}}$. The wave vector transfer $\vec{Q}=\vec{k}-\overrightarrow{k^{\prime}}$ is an important physical characteristic of the experiment. The incoming wave depends on the flux $\Phi_{0}$. A detector records the scattering intensity $I_{\mathrm{sc}}$ within the solid angle $\Delta \Omega$. The probed volume is indicated by the grey disc with a black, dotted frame. In case of kinematical (elastic) scattering $|\vec{Q}|=2|\vec{k}| \sin (\theta)$. 
However, the electrons in an atom are bound necessitating the frequency (wavelength) dependent dispersion or resonant scattering corrections:

$$
f(\vec{Q}, \omega)=f^{0}(\vec{Q})+f^{\prime}(\omega)-i f^{\prime \prime}(\omega)
$$

The corrections also allow for the determination of the effect of bound K-shell electrons of the atoms. Below such a K resonance, the scattering amplitude needs to be reduced in comparison to the Thomson value, yielding negative values for $f^{\prime}(\lambda)$. Notably, the relative strength of $f^{\prime}$ in comparison to $f^{0}$ increases with increasing wave vector. The correction $f^{\prime \prime}$ represents the dissipation of energy in the scattering event, due to photoelectric absorption.

In order to obtain a macroscopic interpretation of the atomistic approach, one can consider the complex dielectric function $\epsilon / \epsilon_{0}$ of a single element material with atom number density $\rho_{a} \mid 161$ :

$$
\epsilon / \epsilon_{0}(\omega)=1-\frac{r_{0} \lambda^{2}}{\pi} \rho_{a} \sum_{j} \frac{\omega^{2} F_{j}}{\left(\omega^{2}-\omega_{j}^{2}\right)+i \gamma_{j} \omega} .
$$

Here, the interaction of the X-rays with bound electrons is described by a dampedoscillator model, where $\omega_{j}, \gamma_{j}$ and $F_{j}$ denote the $\mathrm{j}^{\text {th }}$ resonance frequency, damping constant and oscillator strength, respectively.

Using the identification for the refractive index (cf. subsection 1.1) and equation 1.51 , one obtains 161 :

$$
\begin{aligned}
n(\omega)=\sqrt{\epsilon / \epsilon_{0}} & \approx 1-\frac{r_{0} \lambda^{2}}{2 \pi} \rho_{a} \sum_{j} \frac{\omega^{2} F_{j}}{\left(\omega^{2}-\omega_{j}^{2}\right)+i \gamma_{j} \omega} \\
& =1-\frac{r_{0} \lambda^{2}}{2 \pi} \rho_{a}\left(f_{1}^{0}-i f_{2}^{0}\right) \\
& \equiv 1-\delta+i \beta,
\end{aligned}
$$

where $f_{1}^{0}$ denotes the real and and $f_{2}^{0}$ denotes the imaginary part of the scattering length $(\vec{Q}=0)$ of equation 1.50 . Additionally, in the last line the notation for the complex index of refraction, that is convenient in the $\mathrm{X}$-ray regime, has been introduced. This notation owes to the fact that the material constants $\delta$ and $\beta$ are typically very small. In the hard X-ray regime $\delta$ is on the order of $10^{-6}$ for proteins and $\beta$ is about two orders of magnitude below (cf. table 1.3).

In the important case of a medium with a mixture of elements one needs to sum over the elements which yields the decrement of the real part of the refractive index $46,95,124,255$ :

$$
\begin{aligned}
\delta & =\frac{r_{0} \lambda^{2}}{2 \pi} \sum_{j} \rho_{a_{j}} f_{1 j}^{0}=\frac{r_{0} \lambda^{2}}{2 \pi} \sum_{j} \frac{N_{j}}{V}\left(Z_{j}+f_{j}^{\prime}\right) \\
& =\frac{r_{0} \lambda^{2}}{2 \pi}\left(\rho_{e}(\vec{r})+\rho_{m}(\vec{r}) \sum_{j} q_{j} \frac{f_{j}^{\prime}}{A_{j}}\right),
\end{aligned}
$$


Table 1.3: The table lists some $\delta, \beta$ values for the model protein $\mathrm{H}_{50} \mathrm{C}_{30} \mathrm{~N}_{9} \mathrm{O}_{10} \mathrm{~S}_{1}$ (mass density $\rho_{m}=1.35 \mathrm{~g} / \mathrm{cm}^{3}[141$ ) as a function of the X-ray energy. Values have been obtained from [125].

\begin{tabular}{cccc}
\hline$E[\mathrm{keV}]$ & $\delta\left[10^{-6}\right]$ & $\beta\left[10^{-8}\right]$ & $\delta / \beta$ \\
\hline & & & \\
6.2 & 7.83366249 & 4.57656633 & 171 \\
7.9 & 4.81518464 & 1.73911445 & 277 \\
13.8 & 1.57349984 & 0.190856819 & 824
\end{tabular}

where $\rho_{e}$ is the electron density, $\rho_{m}$ is the mass density, $q_{j}=m_{j} / m$ denotes the mass fraction of the $\mathrm{j}^{\text {th }}$ element and $A_{j}=m_{j} / N_{j}$ is the atomic weight. At this point, we note that the correction terms $f_{j}^{\prime}$ can be safely ignored if the probing wavelength does not correspond to resonances. Therefore, an important result is:

$$
\delta \approx \frac{r_{0} \lambda^{2}}{2 \pi} \rho_{e}(\vec{r})
$$

Literally, the equation states that the (real) refractive index decrement is proportional to the electron density of the probed material. Considering now the case of light elements where the approximation $Z_{j} / A_{j} \approx 1 / 2 u$ holds $\left(u=1.661 \cdot 10^{-24} \mathrm{~g}\right.$ is the atomic mass unit), one obtains:

$$
\frac{\rho_{e}}{\rho_{m}}=\frac{\sum_{j} N_{j} Z_{j}}{m}=\sum_{j} q_{j} \frac{Z_{j}}{A_{j}} \approx \frac{1}{2 u} \sum_{j} q_{j}=\frac{1}{2 u} .
$$

Hence, the electron density can be identified with the mass density 95$]$ :

$$
\delta \approx \frac{r_{0} \lambda^{2}}{4 \pi u} \rho_{m}(\vec{r})
$$

In practical units this yields 46

$$
\delta \approx 1.35 \cdot 10^{-6} \lambda^{2}[\AA] \rho_{m}\left[\mathrm{~g} / \mathrm{cm}^{3}\right],
$$

for a material with macroscopic mass density $\rho_{m}$. In addition, we note that for a single element

$$
\delta \propto \lambda^{2} Z \propto E^{-2} Z .
$$

Likewise, the imaginary part of the refractive index corresponding to photoelectric absorption can be identified: 


$$
\beta=\frac{r_{0} \lambda^{2}}{2 \pi} \sum_{j} \rho_{a, j} f_{j}^{\prime \prime}
$$

where the absorption coefficient $\mu$ is given in terms of the element specific absorption cross-sections $\sigma_{a, j}$ (overall absorption cross-section $\sigma_{a}$ ) by

$$
\mu=2 k \beta=2 r_{0} \lambda \sum_{j} \rho_{a, j} f_{j}^{\prime \prime}=\sum_{j} \rho_{a, j} \sigma_{a, j} \equiv \sigma_{a} .
$$

Away from resonances, one finds experimentally $[6]$ that

$$
\beta \propto \lambda^{4} Z^{4} \propto E^{-4} Z^{4} .
$$

Hence, one obtains the approximate behaviour:

$$
\frac{\delta}{\beta} \propto \frac{E^{2}}{Z^{3}},
$$

which indicates the importance of $\delta$ in comparison to absorption events at high energies.

Without going into the quantum mechanical subtleties (cf. e.g. [6,31]), the occurrence of characteristic X-ray fluorescence radiation due to photoelectric absorption needs to be addressed. In an atom, electrons are bound in certain energy states (shells) whereas the lowest (energetically favourable) state is the K-shell (cf. Fig. 1.8). Assuming that the energy of an incoming X-ray photon is sufficiently above the binding energy of the electron, a channel for photoelectric absorption becomes available. These channels are visible as steps or edges in the $1 / e$ penetration depth as a function of photon energy (cf. Fig. 1.10). In such an event, the energy of the incoming photon is absorbed by the bound electron which undergoes an energetic transition to the continuum. A free energy state is thus created in the shell (a 'hole'). Relaxation of the ionized atom can be reached by different processes. Typically, electrons from energetically higher shells fill created holes below. The excess of transition energy can be released either by emitting the characteristic fluorescence radiation or by expelling other electrons occupying an even higher energy state 6 . The latter process is known as 'Auger' electron emission. The dominant fluorescent radiation that can be induced with X-rays within the energy range of about $2-16 \mathrm{keV}$ is the so-called $K_{\alpha}$ and $K_{\beta}$ radiation, where the Greek subscript denotes a transition from a L- to K-shell and from a M- to K-shell. A detailed list of X-ray binding energies and emission lines can be found in 302 .

\subsubsection{The Projection Approximation}

The projection approximation allows to determine the field directly behind a sufficiently thin object (along the optical axis) $n(\vec{x}) \neq 1$ only for $0 \leq z \leq z_{1}$ (cf. Fig. 1.9). For this reason, one assumes that the incident wave-field $\Psi_{0}(\vec{x})$ can 


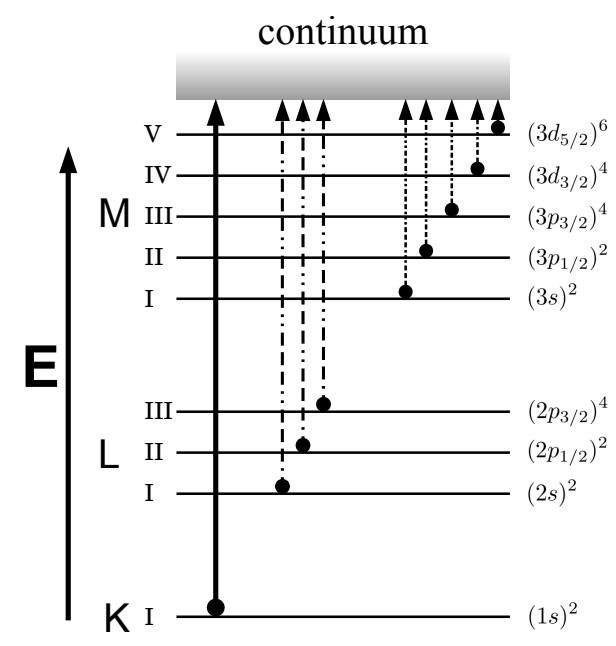

Figure 1.8: The figure illustrates the energy states of bound electrons. The most favourable and energetically lowest state of an electron is the $(1 s)^{2}$ (K-shell). An excitation into the continuum can be induced by photoelectric absorption of an X-ray photon whose energy is higher than the binding energy. The freed energy due to an electron that fills the created hole yields characteristic (fluorescence) K-, L- or M-shell radiation. Increasing with atomic number, phosphorus is the first element exhibiting $\mathrm{K}$-shell radiation above $2 \mathrm{keV}[302]$. The shell nomenclature is $\left(n l_{j}\right)^{2 j+1} . n, l$ and $j$ denote principal, orbital and angular momentum, respectively. Adapted from [6].

be considered 'beam-like' along this section. In particular, it shall be decomposed into a term $\exp (i k z)$ oscillating rapidly in the z-direction and a term $\psi(\vec{x})$ that does not, i.e. $\partial_{z}^{2} \psi(\vec{x}) \ll 1: \Psi_{0}(\vec{x})=\exp (i k z) \psi(\vec{x})$. Using this ansatz in equation 1.2 yields the 'inhomogeneous paraxial equation':

$$
\left(2 i k \partial_{z}+\partial_{x}^{2}+\partial_{y}^{2}+k^{2}\left(n^{2}(\vec{x})-1\right)\right) \psi(\vec{x})=0 .
$$

The additional assumption of 'stream-like' propagation along the section necessitates that the term $\left(\partial_{x}^{2}+\partial_{y}^{2}\right) \psi(\vec{x})$ is small compared to $k^{2}$. Now no coupling is possible between the $\mathrm{x}$ - and $\mathrm{y}$ - components of the field. One obtains the differential equation for a field $\psi(\vec{x})$ that is projected onto an object $n(\vec{x})$ :

$$
\partial_{z} \psi(\vec{x})=\frac{k}{2 i}\left(1-n^{2}(\vec{x})\right) \psi(\vec{x}) .
$$

The solution is

$$
\psi\left(x, y, z_{0}\right)=\exp \left(\frac{k}{2 i} \int_{0}^{z_{0}}\left(1-n^{2}(\vec{x})\right) d z\right) \psi(x, y, 0)
$$




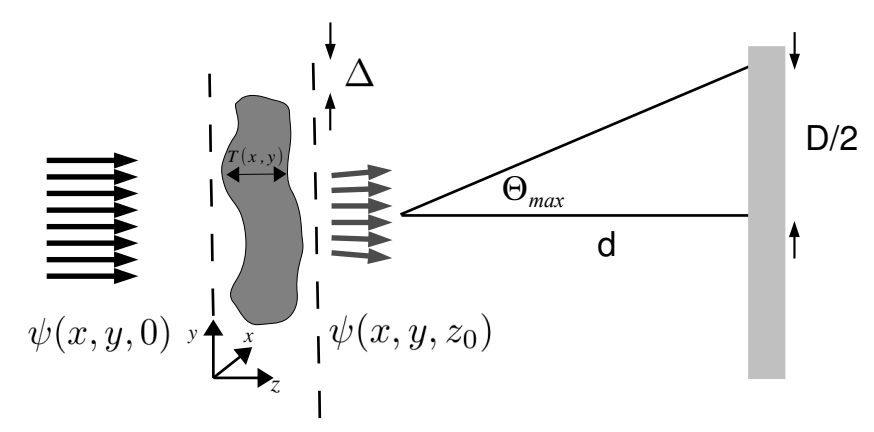

Figure 1.9: In the projection approximation the field directly behind a sample is given by the expression $\psi\left(x, y, z_{0}\right)=\exp \left(\frac{k}{2 i} \int_{0}^{z_{0}}\left(1-n^{2}(\vec{x})\right) d z\right) \psi(x, y, 0)$. The object is imprinted by its projected thickness $T(x, y)$ onto the incident wave-field $\psi(x, y, 0)$. Here, the typical scattering geometry is depicted where the resolution $\Delta$ depends on the highest scattering angle $\theta_{\max }$ that can be detected on a detector $\mathrm{D}$.

The approximation $1-n^{2} \approx 2(\delta-i \beta)$ thus yields

$$
\begin{aligned}
\psi\left(x, y, z_{0}\right)= & \exp \left(-i k \int_{0}^{z_{0}} \delta(x, y, z) d z\right) \exp \left(-k \int_{0}^{z_{0}} \beta(x, y, z) d z\right) \\
& \psi(x, y, 0) .
\end{aligned}
$$

Consequently, the object imprints itself onto the incoming beam $\psi(x, y, 0)$ by phase shifts

$$
\varphi(x, y)=-k \int_{0}^{z_{0}} \delta(x, y, z) d z
$$

and attenuation. An important case arises if the elemental composition does not change along the z-direction. This yields

$$
\varphi(x, y)=-k \delta(x, y) T(x, y)
$$

where $T(x, y)=\int_{\mathrm{Obj}} d z$ denotes the projected thickness. Experimental expectations for phase shift and attenuation of biological material have been calculated using tabulated data for $\delta$ and $\beta$. The results are presented in figure 1.10 and table 1.4. Evidently, phase contrast becomes more important than absorption in the hard X-ray regime above approx. $2 \mathrm{keV}$. 
Table 1.4: The table presents calculated penetration lengths for attenuation $z_{1 / e}=$ $\lambda / 4 \pi \beta$ and phase shift (of $\pi$ ) $z_{\pi}=\lambda / 2 \delta$ for the model protein $\mathrm{H}_{50} \mathrm{C}_{30} \mathrm{~N}_{9} \mathrm{O}_{10} \mathrm{~S}_{1}$ (mass density $\rho_{m}=1.35 \mathrm{~g} / \mathrm{cm}^{3} \mid 141$ ) against a background of vacuum and water for specific energies (cf. Fig. 1.10). $z_{1 / e}^{\mathrm{H}_{2} \mathrm{U}}$ corresponds to a $1 / e$ value of the ratio of $I^{\text {protein }} / I^{\mathrm{H}_{2} \mathrm{O}}$, i.e. the attenuation of equal paths through either protein or water are compared.

\begin{tabular}{ccccc}
\hline$E[\mathrm{keV}]$ & $z_{1 / e}^{v a c}[\mu \mathrm{m}]$ & $z_{1 / e}^{\mathrm{H}_{2} 0}[\mu \mathrm{m}]$ & $z_{\pi}^{v a c}[\mu \mathrm{m}]$ & $z_{\pi}^{\mathrm{H}_{2} 0}[\mu \mathrm{m}]$ \\
\hline & & & & \\
6.2 & 348 & 352 & 13 & 35 \\
7.9 & 720 & 739 & 16 & 44 \\
13.8 & 3751 & 4344 & 29 & 76 \\
15.25 & 4967 & 6065 & 32 & 84 \\
& & & & \\
\hline
\end{tabular}

In order to obtain the (full) $3 \mathrm{~d}$ representation one can solve for $\delta$ and $\beta$ tomographically as the integral along the z-direction represents a projection. The validity of the projection approximation depends on the maximum thickness $z_{0}$ of the object. For instance, Weierstall and co-workes [321] require that (cf. also 216])

$$
z_{0}<2 \Delta^{2} / \lambda
$$

where $\Delta$ denotes the smallest length scale that can be resolved in a given experiment. Using the small angle approximation $\sin (\theta) \approx \theta \approx \tan (\theta)$ and the scattering geometry as depicted in figure 1.9 , one also obtains the following relation (cf. equation 1.15):

$$
\begin{aligned}
\Delta & =\frac{\lambda d}{D}=\frac{\lambda}{2} \frac{1}{D / 2 d} \approx \frac{\lambda}{2} \frac{1}{\sin \left(\theta_{\max }\right)}=\frac{\lambda}{2 \mathrm{NA}}, \\
\Rightarrow z_{0} & <2 \Delta^{2} / \lambda=\frac{\lambda}{2 \mathrm{NA}^{2}} .
\end{aligned}
$$

The last expression is typically denoted as the depth of focus (DOF) ${ }^{3}[39,284]$. Importantly, the projection approximation indicates that the object information being encoded in an electric field is essentially $2 \mathrm{~d}$ if a certain resolution limit is not surpassed or if the sample is thinner than a depth of focus. Table 1.5 gives some numerical examples for X-ray energies that are important for this thesis. Notably, the typical thickness of samples that are studied in this work is in the range of $0.5 \mu \mathrm{m}-1.5 \mu \mathrm{m}$ which allows a resolution of about $10 \mathrm{~nm}$ without violating the projection approximation.

${ }^{3}$ Note that the pre-factor for the depth of focus of a Gaussian beam is slightly different. For a Gaussian beam the DOF is defined as two times the Rayleigh length $\tilde{z}$, i.e. $\operatorname{DOF}_{G}=2 \tilde{z}=$ $(4 / \pi) \lambda / 2 \mathrm{NA}^{2}$. 


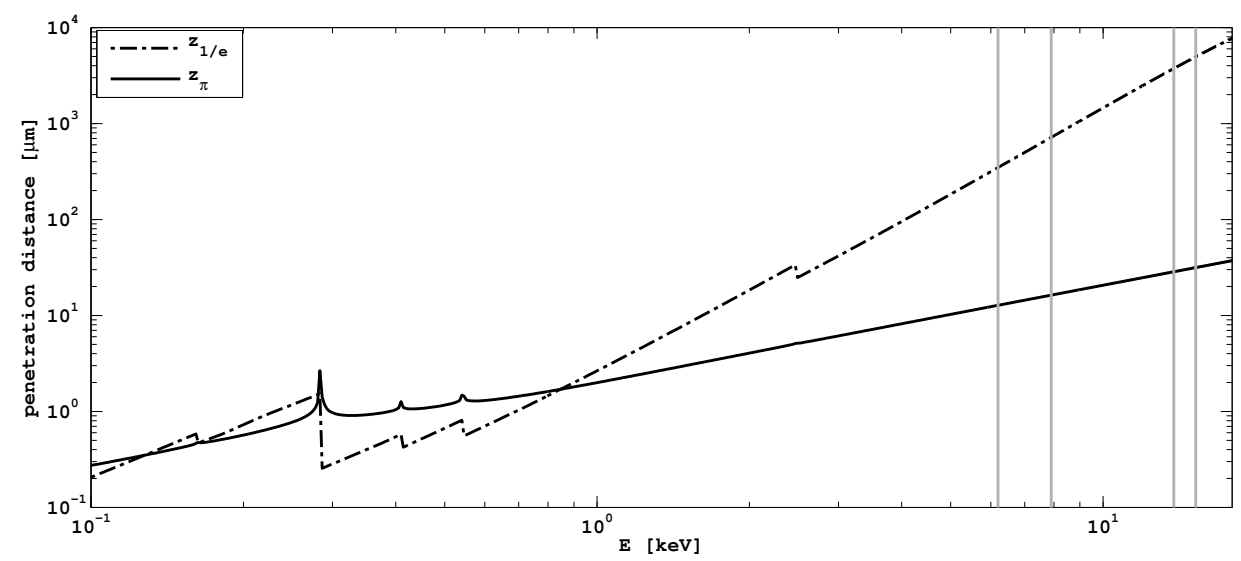

(a)

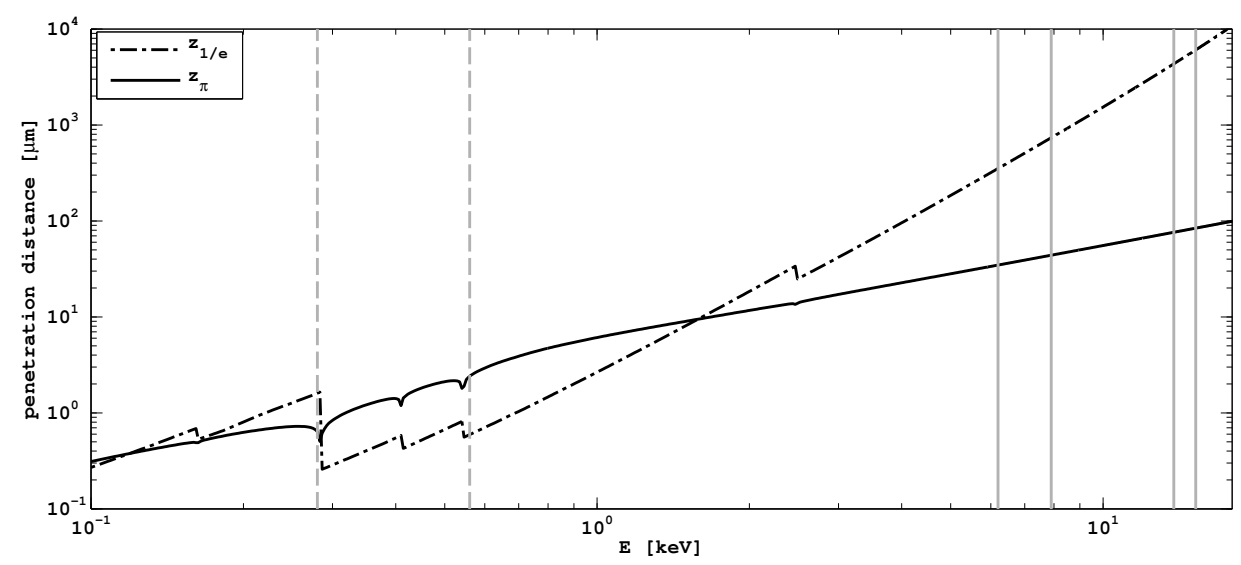

(b)

Figure 1.10: (a) presents calculated penetration lengths for attenuation (dashed-dotted line) to a factor $e^{-1}\left(z_{1 / e}=\lambda / 4 \pi \beta\right)$ and a phase shift (solid line) of $\pi\left(z_{\pi}=\lambda / 2 \delta\right)$ for the model protein $\mathrm{H}_{50} \mathrm{C}_{30} \mathrm{~N}_{9} \mathrm{O}_{10} \mathrm{~S}_{1}$ (mass density $\rho_{m}=1.35 \mathrm{~g} / \mathrm{cm}^{3}|141|$ ) against a background of vacuum. (b) shows the penetration lengths against a background of water (mass density $\rho_{m}=1 \mathrm{~g} / \mathrm{cm}^{3}$ ). $z_{1 / e}^{\mathrm{H}_{2} \mathrm{O}}$ corresponds to a $1 / e$ value of the ratio of $I^{\text {protein }} / I^{\mathrm{H}_{2} O}$, i.e. the attenuation of equal paths through either protein or water are compared. Curves have been calculated using the tabulated data from Henke and co-workers [125]. The vertical, dashed and grey lines indicate the region of the so-called water window which lies between the carbon $(284 \mathrm{eV})$ and oxygen $(540 \mathrm{eV})$ $\mathrm{K}$ absorption edges [161]. In this range absorption contrast is clearly favoured. In the hard X-ray region above $\sim 2 \mathrm{keV}$ phase contrast becomes the dominant contrast (note the point of intersection in (b) at around this energy). The vertical, solid and grey lines indicate experimental energies in this work at $E=6.2,7.9,13.8,15.25 \mathrm{keV}$. Notably, the attenuation is hardly affected by an equally thin layer of water, whereas the phase shift can be expected to be lowered by about one order of magnitude. 
Table 1.5: The table lists the limiting sample thickness $z_{\max }$ in microns for the projection approximation (eqn. 1.72) to hold as a function of X-ray energy $E$ and requested resolution $\Delta$.

\begin{tabular}{ccccc}
\hline$E[\mathrm{keV}] \backslash$ & $\Delta[\mathrm{nm}]$ & 100 & 50 & 10 \\
\hline & & & \\
6.2 & 100 & 25 & 1 \\
7.9 & 127 & 32 & 1.3 \\
13.8 & 223 & 56 & 2.2 \\
15.25 & 246 & 61 & 2.5 \\
& & & \\
\hline
\end{tabular}




\subsubsection{Dose vs. Resolution}

Biological samples are prone to radiation damage which results in morphological changes or structural deterioration 161. Firstly, the damage affects the atomic structure due to photoionisation. Next, ultrastructural changes appear and, finally, larger structural deterioration becomes apparent. On scales $\gtrsim 50 \mathrm{~nm}$, radiation damage is observed as mass loss. Typically, radiation damage is compared with the invested dose, i.e. the deposited energy per unit mass. In case of X-rays with energy $E=h \nu\left(h=6.626 \cdot 10^{-34}\right.$ Js denotes Planck's constant), one finds the surface dose (units Gray) 141

$$
D=\frac{\mu N_{0} h \nu}{\rho_{m}}
$$

where $\mu$ is the absorption coefficient, $N_{0}$ is the number of incident photons per unit area and $\rho_{m}$ is the mass density of the sample. Note that, $\mu N_{0}=-\left.\partial_{z} N_{0} e^{-\mu z}\right|_{z=0}$ corresponds to the number of absorbed photons due to the Beer-Lambert law. Depending on the type of X-ray imaging experiment it is necessary to differentiate between (i) wet and chemically fixed, (ii) frozen hydrated and (iii) dehydrated samples. Reported critical doses, above which damage can be observed, in these categories are e.g. $>10^{5}-10^{6}$ Gray (i), $>10^{8}$ Gray (ii) and $>10^{7}$ Gray $|161|$. In case of metabolically active specimens, the tolerable dose can differ. For instance, it may be higher for bacteria than for fibroblasts 161 . Especially, experiments of category (i) depend on the time scales during which the X-ray data is collected. On time scales $<10 \mathrm{~ms}$ radiation damage may remain localized on a spatial scale of $50 \mathrm{~nm} 323,336$. Here it should be noted that data taken on or below the femtosecond scale, i.e. a scale below which atomic motion due to photoionisation has not started yet, is considered to yield information about the unperturbed structure down to the atomic level. This technique is used at X-ray Free Electron Laser sources (XFELs) and known as 'diffraction before destruction' [40.

However, radiation damage represents an issue for the experiments in this thesis that needs further discussion. It is useful to estimate the dose being necessary to image a certain feature of the sample of size $\Delta$ and one needs to know the maximum tolerable dose. The number of photons that will be scattered into the detector is

$$
P=N_{0} \Delta^{2} \cdot \sigma_{s} / \Delta^{2}=N_{0} \sigma_{s}
$$

where $\sigma_{s}$ denotes the coherent scattering cross section. Howells and co-workers calculated the coherent scattering cross section $\sigma_{s}$ of a small voxel of dimension $\Delta \times \Delta \times \Delta[141$

$$
\sigma_{s}=r_{0}^{2} \lambda^{2}\left|\tilde{\rho}_{e}\right|^{2} \Delta^{4}
$$


where $\tilde{\rho}_{e}=\left(2 \pi / r_{0} \lambda^{2}\right)\left(\left(\delta-\delta_{0}\right)+i\left(\beta-\beta_{0}\right)\right)$ denotes a relative complex electron density (cf. equation 1.57) with respect to some background material (subscript zero). Hence, the invested dose for observing $P$ photons is 141

$$
\begin{aligned}
D & =\frac{\mu P h \nu}{\sigma_{s} \rho_{m}}=\frac{\mu P h \nu}{\rho_{m}} \frac{1}{r_{0}^{2} \lambda^{2}\left|\tilde{\rho}_{e}\right|^{2} \Delta^{4}} \\
& =\frac{\mu P h \nu}{\rho_{m}} \frac{(\lambda / 2 \pi)^{2}}{\left(\delta-\delta_{0}\right)^{2}+\left(\beta-\beta_{0}\right)^{2}} \frac{1}{\Delta^{4}} .
\end{aligned}
$$

Identifying $\Delta$ with the acquired resolution of the scattering experiment leads to the important conclusion, that the necessary dose is proportional to the reciprocal of the resolution to the power of four

$$
D \propto \frac{1}{\Delta^{4}}
$$

In addition, the necessary dose for imaging decreases quadratically with the phase contrast being proportional to the difference $\left|\delta-\delta_{0}\right|$ (neglecting absorption). Until now, there is no measure of the number of photons $P$ that need to be detected for successful phase retrieval. Nevertheless, the Rose criterion [252] is typically used to define the detectable minimum photon number above noise. In case of Poisson noise, the photon count needs to be 25 to be five times higher than the noise as given by the standard deviation $\sigma_{\text {Poisson }}=\sqrt{25}=5$. Examples of dose and fluence requirements have been calculated according to equation 1.79 as presented in 141 . The two examples protein against a background of water or vacuum are presented in figure 1.11. In the hard X-ray region it can be seen that the required dose for imaging of protein in vacuum lies about one order of magnitude below the curve for protein in water but otherwise remains effectively constant. Reducing the desired resolution from $10 \mathrm{~nm}$ to $50 \mathrm{~nm}$ alleviates the dose/ fluence requirements by approximately three orders of magnitude. Here, it should be noted that the contrast of a given feature that needs to be reconstructed, i.e. the grey levels, also affect the necessary dose requirements. Schropp and Schroer calculated that the increase in dose follows the reciprocal of the contrast to the power of two [269]. For instance, the increase in dose from one grey level to ten shades of grey is by a factor $1 /(0.1)^{2}=100$.

As explained above, dose affects the sample morphology and structure on different time- and length-scales. Howells and co-workers summarized many experimental observations of the maximum tolerable dose. As a conclusion they obtained an approximate formula 141:

$$
D_{\max }[\mathrm{Gy}] \approx 1 \times 10^{8} \times \Delta[\mathrm{nm}]
$$


The formula holds in the range $\Delta=10-100 \mathrm{~nm}$. Importantly, at $\Delta=10 \mathrm{~nm}$ the curve intersects with the required dose for imaging (equation 1.79). Therefore, the resolution limit for imaging of cryo-protected cellular features against a background of water is considered to be $10 \mathrm{~nm}$.

Here it should be noted, that equations $1.79,1.81$ have been obtained for the case of the far-field regime. Consequently, imaging in e.g. the Fresnel regime may have slightly different requirements (cf. 14,241]). In addition, the theoretical derivation for the minimal necessary dose for imaging (equation 1.79) was derived for a single resolution element. According to the dose fractionation theorem 123 the dose for a full $3 \mathrm{~d}$ reconstruction at a given resolution is about the same as the required dose for a $2 \mathrm{~d}$ reconstruction with an equal resolution. By sharing the same dose over all projections, the single $2 \mathrm{~d}$ projections clearly have a lower resolution but the full $3 \mathrm{~d}$ reconstruction that is formed by the superposition of the $2 \mathrm{~d}$ data improves to the dose-defined level. The same is true for the case of diffraction data based imaging techniques: in an analogous way the transition from the considered voxel element to the $3 \mathrm{~d}$ specimen can be made as has been pointed out by Howells and co-workers 141 . 


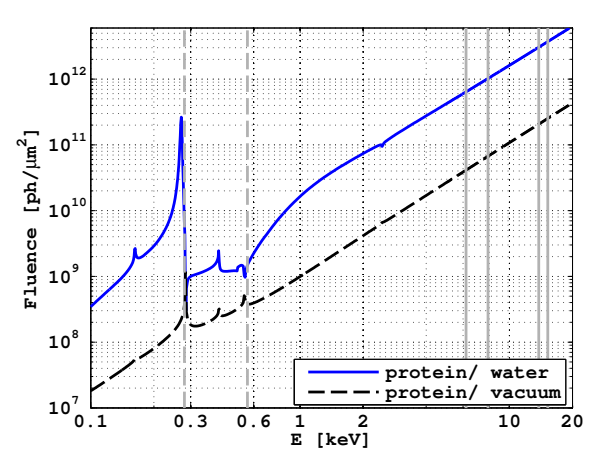

(a) $\Delta=10 \mathrm{~nm}$

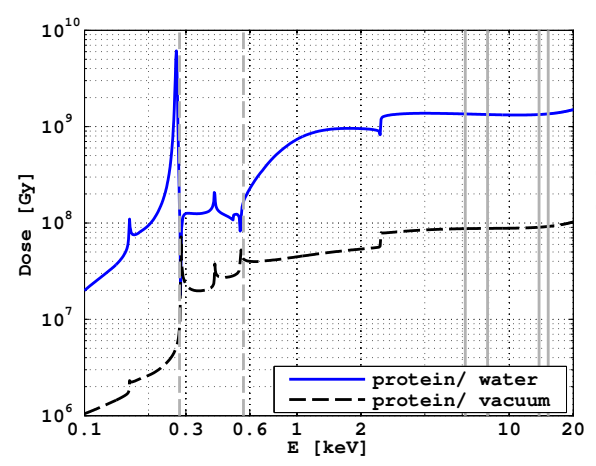

(c) $\Delta=10 \mathrm{~nm}$

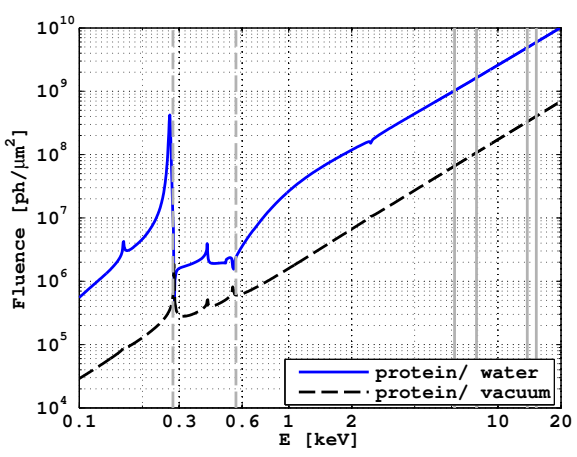

(b) $\Delta=50 \mathrm{~nm}$

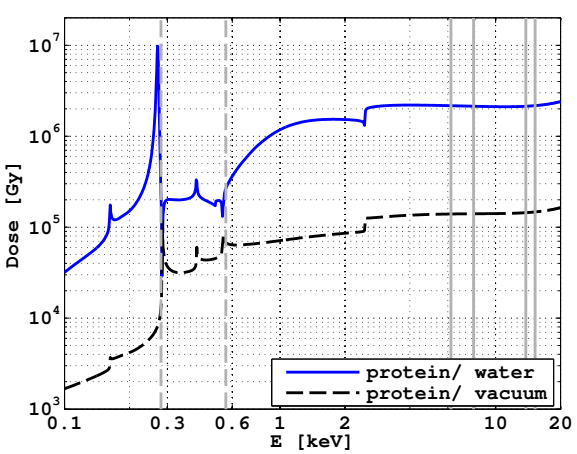

(d) $\Delta=50 \mathrm{~nm}$

Figure 1.11: The figure shows fluence and dose curves that satisfy the Rose criterion for a required resolution of $\Delta=10 \mathrm{~nm}(\mathbf{a}),(\mathbf{c})$ and $\Delta=50 \mathrm{~nm}(\mathbf{b}),(\mathbf{d})$ according to equation 1.79. The solid, blue curves correspond to protein $\mathrm{H}_{50} \mathrm{C}_{30} \mathrm{~N}_{9} \mathrm{O}_{10} \mathrm{~S}_{1}$ (mass density $\rho_{m}=1.35 \mathrm{~g} / \mathrm{cm}^{3}[141]$ ) against a background of water (mass density $\left.\rho_{m}=1 \mathrm{~g} / \mathrm{cm}^{3}\right)$. The vertical, dashed and grey lines indicate the region of the 'water window' 161]. In this range the dose/ fluence requirements for the case of background water is significantly reduced. In the hard X-ray region above $\sim 2 \mathrm{keV}$ the dose/ fluence requirements remain approximately constant. The vertical, solid and grey lines indicate experimental energies in this work at $E=6.2,7.9,13.8,15.25 \mathrm{keV}$. Tabulated values for the complex index of refraction are due to 125 . 


\subsection{Imaging with X-Rays}

Firstly, a short recapitulation of direct imaging contrast mechanisms (STXM) that are readily available in many $\mathrm{X}$-ray scanning set-ups is given. Next, Coherent Diffractive Imaging (CDI) is introduced on the basis of the 'phase problem' of isolated specimens. Sampling issues are discussed and as an example the algorithm that was applied for obtaining results within this thesis (section 3.5) is outlined. The following topic is Ptychographic Coherent Diffractive Imaging (PCDI). In addition to a review of recent achievements using Ptychography, the 'ePIE' algorithm is described. The section closes with a very brief treatise of tomography.

\subsubsection{Direct Methods}

Before turning the scope on iterative phase retrieval methods, the important contrast mechanisms 'absorption', 'differential phase contrast' and 'darkfield' will be discussed $207,208,295$. These contrast mechanisms are very useful if the object is raster-scanned with a small illuminating beam. Here, a mathematical derivation similar to the one by Thibault and colleagues $[295]$ will be presented.

We start with an incident illumination function $P(\vec{\rho})$ and an object $O(\vec{\rho})$ in a plane $z=0, \vec{\rho}=(x, y)$. Assuming the projection approximation (equation 1.68 ) to be valid yields the exit surface wave at the illumination position $\vec{\rho}_{i}$ : $\psi\left(\vec{\rho}, \vec{\rho}_{i}\right)=O(\vec{\rho}) P\left(\vec{\rho}-\vec{\rho}_{i}\right)$. In addition, let the object be determined by its complex index of refraction according to equation 1.69 . The integrated phase and absorption are thus $\Phi(\vec{\rho})=k \int \delta(\vec{x}) d z$ and $\mu(\vec{\rho})=2 k \int \beta(\vec{x}) d z$, respectively. The contrast mechanisms of absorption and differential phase contrast are derived from the zeroth and first moments of the $2 \mathrm{~d}$ far-field intensity distribution $I\left(\vec{q}, \vec{\rho}_{i}\right)$. Considering the case of a pure absorption sample $(\Phi \equiv 0)$ yields the zeroth moment:

$$
\begin{aligned}
\langle I\rangle\left(\vec{\rho}_{i}\right) & =\iint_{-\infty}^{\infty}|\mathcal{F}\{\psi\}|^{2} d q_{1} d q_{2}=\iint_{-\infty}^{\infty}|\psi|^{2} d \rho_{1} d \rho_{2} \\
& =\iint_{-\infty}^{\infty}\left|O(\vec{\rho}) P\left(\vec{\rho}-\vec{\rho}_{i}\right)\right|^{2} d \rho_{1} d \rho_{2} \\
& =\iint_{-\infty}^{\infty} \exp (-\mu(\vec{\rho}))\left|P\left(\vec{\rho}-\vec{\rho}_{i}\right)\right|^{2} d \rho_{1} d \rho_{2} . \\
\Rightarrow\langle I\rangle\left(\vec{\rho}_{i}\right) & =|P|^{2} \star \exp (-\mu)\left(\vec{\rho}_{i}\right)
\end{aligned}
$$

In the first line Parselval's identity has been used 25 . In words, the result shows that the diffraction pattern from each scan position is related to the integrated absorption by a convolution with the intensity of the illumination function. Hence, the resolution of the image that is obtained by rastering the object along the positions $\overrightarrow{\rho_{i}}$ is limited by the beam size. 
In case of a centrosymmetric beam, i.e. $P(\vec{\rho})=P(-\vec{\rho}),{ }^{4}$ and assuming constant absorption $\mu(\vec{\rho})=\mu_{0}=$ const., one finds for the first moments $\left\langle q_{\alpha} I\right\rangle\left(\vec{\rho}_{i}\right)(\alpha=1,2)$ 295 :

$$
\Rightarrow\left\langle q_{\alpha} I\right\rangle\left(\vec{\rho}_{i}\right)=|P|^{2} \star \partial_{\alpha} \Phi\left(\vec{\rho}_{i}\right), \quad \alpha=1,2 .
$$

The first moments thus yield information about local phase gradients. Darkfield contrast is obtained by calculating $\langle\chi I\rangle\left(\vec{\rho}_{i}\right)$ :

$$
\langle\chi I\rangle\left(\vec{\rho}_{i}\right)=\iint_{-\infty}^{\infty} \chi|\mathcal{F}\{\psi\}|^{2} d q_{1} d q_{2},
$$

where $\chi$ denotes a mask function that is unity in a certain frequency region and zero elsewhere. Therefore, $\chi$ acts as a filter for certain spatial frequencies within the spectrum of $\psi$, that is, part of the object at position $\vec{\rho}_{i}$ as defined by the illuminating envelope. Typically, a high-pass filter is used that blocks the central beam on the detector. Hence, in the integration only the scattering signal is summed up. Thereby, a contrast that is sensitive to sharp edges is created. In principle, the function $\chi$ can be arbitrarily defined. For instance, the contrast with respect to a certain Bragg reflection can be maximised by restricting the mask to the location of the Bragg peak.

\subsubsection{The Phase Problem}

To date, there is no direct method to measure the phases of the rapidly oscillating wave-field neither in the optical nor in the X-ray regime. Knowledge of the phases is desired as, for instance, it allows inversion of diffraction data but only the intensity of the electromagnetic field can be recorded. This leads to the 'phase problem':

'Given diffraction data (a diffraction pattern or a set of Bragg reflections), find the corresponding phases which satisfy additional constraints.' 293

Due to the similarity between the time-independent $2 \mathrm{~d}$ Schrödinger equation for quantum particles without spin and the Helmholtz equation 1.2 it is not surprising to find Pauli the first one to encounter the phase problem 223 . In terms of the optical terminology, the 'Pauli problem' is: Given two intensity measurements of two planes (e.g. object plane and the diffraction plane), what are the phases of the field? In the field of microscopy with electrons and visible light, the 'Pauli problem' occurred to Gerchberg and Saxton 92 who found the first numerical algorithm that can solve this kind of phase problem.

From an experimental point of view, one needs to find the $N$ phases corresponding to the unknown samples of the object function $O(\vec{\rho}) \in \mathbb{C}$, which are constraint by the measured intensity data

$$
I\left(\vec{q}_{n}\right)=\left|\mathcal{F}\{O(\vec{\rho})\}\left(\vec{q}_{n}\right)\right|^{2}, \quad n=1, \ldots, N
$$

\footnotetext{
4 For instance, assuming a Gaussian beam intensity.
} 
and additional a priori constraints such as a known extent of the object, i.e. the support $S$. It is illustrative to write the equation in terms of the discrete Fourier transform 187

$$
I\left(\vec{q}_{n}\right)=\left|\sum_{\vec{\rho} \in S} O(\vec{\rho}) \exp \left(i \vec{\rho} \cdot \vec{q}_{n}\right)\right|^{2}=\sum_{\vec{\rho}, \vec{\rho}^{\prime} \in S} c_{\rho, \rho^{\prime}}\left(\vec{q}_{n}\right) O(\vec{\rho}) O^{*}\left(\vec{\rho}^{\prime}\right),
$$

where $S$ denotes the support of the object. Each datum point $I\left(\vec{q}_{n}\right)$ corresponds to one ellipsoid solution of possible objects $O(\vec{\rho})$. Taken all datum points together, one thus searches the intersection of the $N$ ellipsoids as defined by equation 1.86 . However, a solution is not easily obtained in general.

\subsubsection{Coherent Diffractive Imaging}

The 'phase problem' and the search for solutions with respect to non-crystalline specimens has been the topic of many scientific works in the past in order to establish a new microscopy technique. The classical experimental approach is to illuminate an isolated, non-crystalline sample with a coherent plane wave and record the resulting far-field diffraction pattern with sufficiently high sampling (oversampling). The phases corresponding to the diffraction data are found by using numerical algorithms, which are similar to the one outlined by Gerchberg and Saxton $[92]$. The algorithms project the current iterate onto the amplitudes of the measured diffraction pattern and some a priori knowledge in the object plane (cf. Fig. 1.12). In the ideal case, the iterate reaches a fixed point at which the constraints of both the measured data and the additional input are satisfied. Intended as a method for high resolution imaging (diffraction limited), the methodology was termed Coherent Diffractive Imaging (CDI). The first experimental demonstration of CDI was achieved by Miao and co-workers using (soft) coherent X-ray radiation with wavelength $\lambda=1.7 \mathrm{~nm} 197$. The imaged sample was a micron sized arrangement of gold dots with a diameter of about $100 \mathrm{~nm}$, which shows the letters 'abcdef'.

Good introductions can be found in text book literature such as 6223 and reviews [216, 243, 297] and [198], where the reader finds a treatment of the crystallographic phase problem. The reader who is interested in practical introductions with an emphasis on the experimental side is referred to the doctoral theses $95,108,267,293$. 


\section{sample plane}

\section{detection plane}
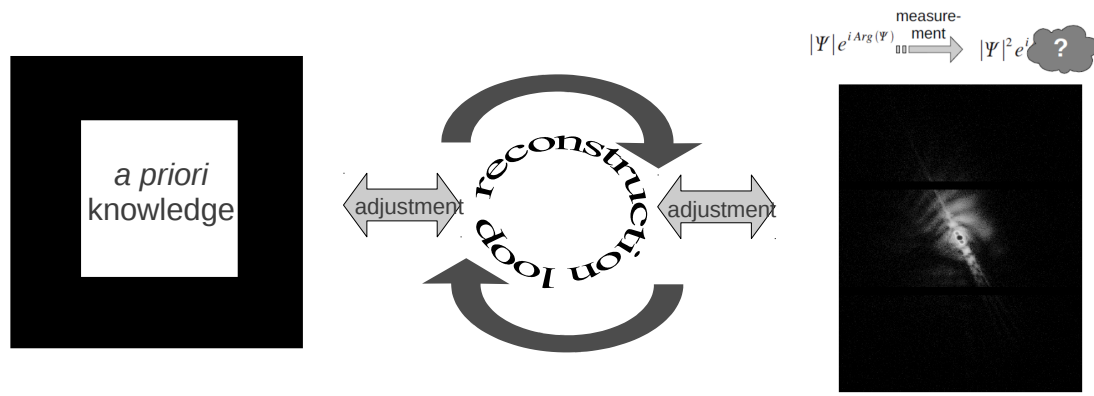

Figure 1.12: Sketch of the phase retrieval scheme. In the detection plane only the diffracted intensity can be measured. Phases corresponding to the underlying electric field are 'lost'. An inversion of the diffraction pattern can be achieved by iteratively cycling between detection plane and object plane. The current iterate is projected onto the measured data (detection plane) and some a priori constraint (object plane).

\subsubsection{Sampling in CDI}

The sampling rate which is given by the detector pixel size is an important parameter for CDI. According to the Whittaker-Shannon sampling theorem, the intensity of the measured diffraction pattern needs to be sampled finely enough to prevent loss of information (cf. equation 1.12, p. 12). The sampling theorem also requires the Fourier transform of the sampled function to be band-limited. Consequently, the Fourier transform of the measured diffraction pattern needs to be band-limited. In the far-field, the intensity is proportional to the modulus squared of the Fourier transform of the sampled function. According to the 'Autocorrelation theorem' (equation 1.146, p. 191) one finds

$$
\mathcal{F}\{I\}=\mathcal{F}\left\{\mathcal{F}\{\psi\} \mathcal{F}\{\psi\}^{*}\right\}=\psi \circledast \psi(-\vec{\rho}),
$$

which is essentially the autocorrelation function of $\psi$. In this case, band-limitation is thus equivalent to band-limitation of the autocorrelation function. The rectangular support (band-pass) enclosing the autocorrelation function needs to be twice as large as the support of $\psi$ (cf. [83,293|). The (Whittaker-Shannon) sampling requirement becomes thus 293 :

$$
\Delta \nu_{i} \leq \frac{1}{2 L_{i}},
$$

where $L_{i}$ are the side-lengths of the support in the $i=x, y$ directions. In terms of the pixel dimensions $\Delta x_{i}^{\prime}$ the sampling condition is equivalent to

$$
\Delta x_{i}^{\prime} \leq \frac{\lambda d}{2 L_{i}}
$$


because $\Delta \nu_{i}=\Delta x_{i}^{\prime} / \lambda d$ with $d$ denoting the distance between the sample and the detection plane. In terms of linear dimensions of the probed object, the WhittakerShannon sampling condition yields e.g. $L_{i} \lesssim 2 \mu \mathrm{m}$ for a detector pixel size of $\Delta x_{i}=172 \mu \mathrm{m}$, an X-ray energy of $E=7.9 \mathrm{keV}$ and a distance of $d=5 \mathrm{~m}$ between sample and detection plane ${ }^{5}$. At a lower energy of $E=6.2 \mathrm{keV}$ and a distance of $d=7 \mathrm{~m}$ this yields $L_{i} \lesssim 4 \mu \mathrm{m}^{6}$. The sampling condition representing a maximum sample dimension can be physically understood as the size of the smallest features of the far-field diffraction pattern ('speckles') are proportional to the reciprocal of the object dimensions.

Based on a counting argument of the number of measured data points (equations to solve) and unknown variables, Miao and colleagues defined the so-called 'oversampling ratio' $\sigma 195$ :

$$
\sigma:=\frac{\text { total pixel number }}{\text { unknown-valued pixel number }}=\frac{\mathrm{A}_{\mathrm{FOV}}}{\mathrm{A}_{\mathrm{SUP}}},
$$

where in the last step $\sigma$ has been expressed in the quantities that are typically used for CDI, namely the area of the field of view and the area of the support of the isolated specimen. In principle, the phase problem should be solvable if $\sigma>2$. Miao and colleagues pointed out that an increasing dimensionality of the object reduces the oversampling in each direction. The oversampling of the magnitude of the Fourier transform of the object needs to be $>2$ for a $1 \mathrm{~d}$ object but $>\sqrt{2}$ in each direction for a $2 \mathrm{~d}$ object and $>2^{1 / 3}$ in each direction for a 3 d object $|195|$. However, the oversampling ratio does not account for all kinds of symmetries in the data. A higher oversampling ratio does not necessarily lead to improved phase retrieval conditions. For this reason, Elser and Millane defined the 'constraint ratio' 744 :

$$
\begin{aligned}
\Omega & :=\frac{\text { number of independent autocorrelation coefficients }}{\text { number of independent object coefficients }} \\
& =\frac{1}{2} \frac{A_{\text {Auto }}}{A_{\mathrm{SUP}}}
\end{aligned}
$$

where $\mathrm{A}_{\text {Auto }}$ denotes the area of the autocorrelation support. Elser and Millane stated that for cases where $\Omega$ is large a successful reconstruction is likely but $\Omega>1$ may already be sufficient $[74]$. The constraint ratio offers a good understanding why different support shapes may strongly influence the reconstruction $84-86$. For instance, a rectangular support yields $\Omega=2$ whereas a triangular support has $\Omega=3[74$. . More examples can be found in 293$]$. Note that in case of ptychography the support is determined by the envelope of the beam at each scan position. Moreover, the oversampling requirement may also be significantly alleviated due to the very stringent overlap constraint of the ptychographic data $[71$.

\footnotetext{
$\overline{5}$ These values correspond to a typical set-up at the P10 beamline at PETRAIII.

6 These values correspond to a typical set-up at the cSAXS beamline at the SLS.
} 


\subsubsection{Phase Retrieval Algorithms for Isolated Objects}

The first practical phase retrieval algorithm (GS) for isolated non-crystalline specimens was developed by Gerchberg and Saxton in the 1970s 92 . Since then many different algorithms such as the prominent Error-Reduction (ER) [82] and the Hybrid-Input-Output (HIO) 86 have been presented. More recent algorithms are the Difference-Map (DM) $\mid 73$, the Hybrid Projection Reflection algorithm (HPR) [18 and the Relaxed Averaged Alternating Reflection algorithm (RAAR) [177]. Discussions about the algorithms can be found in $[17,83,86,128,187,288$. The algorithm that is used in section 3.5 (p. 161) is a mixture of GS and HIO (mHIO) and was first presented by Klaus Giewekemeyer $[94$. It has been proven very useful for imaging of microorganisms such as bacteria 14,94 .

Let $S \subset \mathbb{R}^{2}$ be the support of an isolated specimen, $\chi_{n}(\vec{\rho}) \in \mathbb{C}^{2}$ be the current estimate of the object in the object plane and $\tilde{\chi}_{n}\left(\overrightarrow{\rho^{\prime}}\right) \in \mathbb{C}^{2}$ be the current estimate which was propagated (in the Fresnel regime) to the detection plane:

$$
\tilde{\chi}_{n}\left(\overrightarrow{\rho^{\prime}}\right)=\mathfrak{F}_{\mathfrak{Q}}\left\{\chi_{n}\right\}
$$

Due to noise in the data, it is convenient to carry out a soft projection onto the measured data $\bar{I} 97$

$$
\tilde{P}_{n}\left\{\tilde{\chi}_{n}\right\}:=\sqrt{\left(1-\frac{D}{d}\right) \bar{I}\left(\overrightarrow{\rho^{\prime}}\right)+\frac{D}{d}\left|\tilde{\chi}_{n}\left(\overrightarrow{\rho^{\prime}}\right)\right|^{2}} \cdot \frac{\tilde{\chi}_{n}}{\left|\tilde{\chi}_{n}\right|}, \quad d \geq D,
$$

where $D$ is a noise dependent relaxation parameter. The noise parameter determines the influence of the measured data on the current estimate by comparing it to the reconstruction error $d$

$$
d^{2}\left(\left|\tilde{\chi}_{n}\left(\overrightarrow{\rho^{\prime}}\right)\right|^{2}\right):=\frac{1}{N} \sum_{\overrightarrow{\rho^{\prime}}}\left(\left|\tilde{\chi}_{n}\left(\overrightarrow{\rho^{\prime}}\right)\right|^{2}-\bar{I}\left(\overrightarrow{\rho^{\prime}}\right)\right)^{2}
$$

where $N$ is the number of measured intensity values and the summation is carried out over all $N$ pixels at positions $\overrightarrow{\rho^{\prime}}$. Note that as long as $d \gg D$ the algorithm 'prefers' the measured data. However, when the error decreases to a level where noise in the data becomes important, the weight of the measured data decreases. The complete modulus operator is conveniently written in the plane of the sample as

$$
P_{M}\left\{\chi_{n}\right\}=\mathfrak{F}_{\mathfrak{Q}}^{-1}\left\{\tilde{P}_{n}\left\{\mathfrak{F}_{\mathfrak{Q}}\left\{\chi_{n}\right\}\right\}\right\}
$$

where $\mathfrak{F}_{\mathfrak{Q}}^{-1}\{\cdot\}$ denotes the back-propagation from the detection to the sample plane. Convergence to a solution, however, necessitates one or more constraints in the plane of the sample. Thereby, a full iteration cycle, $\chi_{n} \rightarrow \chi_{n+1}$, is obtained. The attenuation length for cellular material such as proteins is comparably large (cf. table 1.4 , p. 27). Hence, attenuation of the X-ray beam may be negligible for 
bacterial cells with a size in the range of a few microns, which can be enforced by the following operator

$$
P_{\tilde{G S}}\left\{\chi_{n}, \chi_{n}^{\prime}\right\}=\left(\left|\chi_{n}\right|-\beta\left(\left|\chi_{n}^{\prime}\right|-1\right)\right) \exp \left(i \Phi\left(\chi_{n}^{\prime}, \chi_{n}\right)\right),
$$

where $\beta \in[0,1]$ is a feedback parameter, $\chi_{n}^{\prime}=P_{M}\left\{\chi_{n}\right\}$ and the phase $\Phi\left(\chi_{n}^{\prime}, \chi_{n}\right)$ is obtained as described below. The amplitude is thus slowly pushed towards unity with every iteration step. In addition, a support information is used on the phase

$$
\Phi\left(\chi_{n}^{\prime}, \chi_{n}\right)=\left\{\begin{array}{cc}
\arg \left(\chi_{n}(\vec{\rho})\right)-\gamma \arg \left(\chi_{n}^{\prime}(\vec{\rho})\right) & \forall \vec{\rho} \notin S \\
\min \left(\arg \left(\chi_{n}^{\prime}(\vec{\rho})\right), 0\right) & \forall \vec{\rho} \in S
\end{array} .\right.
$$

The phase outside the support is pushed to zero with a strength that is determined from the value of the feedback parameter $\gamma \in[0,1]$. Additionally, the phase is enforced to become negative within the region of the support. Implicitly, it is assumed that the maximum relative phase shift remains below $\pi$. Now, a full iteration cycle is given by

$$
\chi_{n+1}=P_{\tilde{G S}}\left\{\chi_{n}, P_{M}\left\{\chi_{n}\right\}\right\} .
$$

The algorithm is stopped as soon as $d \leq D$.

\subsubsection{Ptychography}

Ptychography (Greek ' $\pi \tau \nu \xi^{\prime}=$ 'to fold', cf. $\mid 122$ ) is one specific method to solve 'the phase problem' by taking advantage of creating redundancy in the data by illuminating adjacent spots on the sample with a certain overlap (cf. Fig. 1.13). Early ideas of ptychography have been developed in the field of electron microscopy and go back to the work of Hoppe, Stube and Hergel $[137-139$ but the first experimental proof of principle needed more than 20 years to be achieved 210 . Especially, in the field of X-ray microscopy, ptychography has advanced to a powerful tool for imaging on the scale of a few nanometres of artificial test structures in $2 \mathrm{~d}[266,287,330,332]$ and $3 \mathrm{~d}[59,133$ as well as, for instance, biological specimen in $2 \mathrm{~d}[62,97,172$ and $3 \mathrm{~d} \mid 61,109,330$. Moreover, the ptychographic method has also been used for elemental speciation $21,135,285$ and for sequential imaging of chemical reactions $[142]$. More recent ptychographic studies investigate the maximum derivable information from ptychographic data under non-simple experimental conditions for the illuminating wave-field or the object such as position uncertainties $|20,110,182|$, partial coherence and multiple wavelenghts [15, 44,78,299], multiple object planes [181,284] and undersampling of the recorded diffracted intensities $[71]$. In the first place, however, the probably most important result has been the understanding that the ptychographic method not only is able to solve for the encoded object information but also for the illuminating wave-field $183,294,296$ making ptychography a valuable tool for analysing the wave-fields of X-rays $\mid 134,144,159,160,265,268$. In principle, the decoupling of object and illuminating wave-field makes the retrieved object information free of aberrations. 
The algorithms that have been applied in this thesis ${ }^{7}$ are the 'difference-map' (DM) approach 294 and the 'extended ptychographical engine' (ePIE) |183|. This section is dedicated to describe basic algorithmic details of ptychography using the example of the ePIE and follows its publication in 183 . Here, the reader is also referred to a more general review on ptychography by John Rodenburg 251 and the doctoral theses of Klaus Giewekemeyer [95] and Robert Hesse [129]. An overview over some recent experimental applications of ptychography can also be found in 298 .

\section{sample plane}

\section{detection plane}

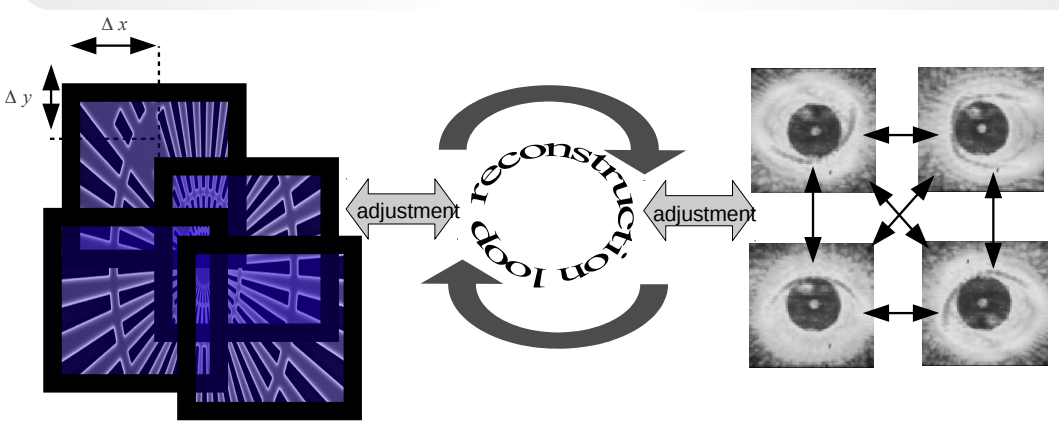

Figure 1.13: Schematic of the ptychographic method. The sample is illuminated with different overlapping illuminated spots by shifting either the object or the illumination function by steps $\Delta x, \Delta y$ in the plane perpendicular to the optical axis. Hence, the phases corresponding to the measured diffracted intensities are not fully independent. In order to estimate the object function and the illuminating wave-field the algorithm cycles through all illuminated positions on the sample and makes updates both in the object plane (real space) and the detection plane (Fourier space).

\subsubsection{The ePIE algorithm}

'ePIE' is an abbreviation for 'extended Ptychographical Iterative Engine'. In contrast to its predecessor [81,249,251, this version is also able to reconstruct the illuminating wave-field simultaneously to the object. The rest of the name is due to the idea that the reconstruction can be performed on-the-fly during recording of the diffraction data 251. Like an engine, you can start at a certain area and reconstruct an image and then proceed by taking into account more and more diffraction patterns from other areas around the beginning.

In case of ptychographic imaging it is a convention to denote the object $O(\vec{r})$ and the illuminating wave-field (probe) $P(\vec{r})$. The scanning of the sample in the plane perpendicular to the optical axis is mathematically equivalent to shift the probe

7 I am very grateful to the staff of the cSAXS beamline who provided me with some helpful routines and the basic ptychographic code of the DM algorithm written by Pierre Thibault. 
by a vector $\vec{R}_{j}(j=1 . . N)$. The exit surface wave $\psi_{j}(\vec{r})$ directly behind the sample is thus given by

$$
\psi_{j}(\vec{r})=O_{j}(\vec{r}) P_{j}\left(\vec{r}-\vec{R}_{j}\right)
$$

Starting with an initial guess for the object and probe, each iteration of the ePIE algorithm goes through all positions in a random sequence $s(j)$ and makes the following updates:

1. apply modulus constraint

2. update exit surface wave

$$
\begin{aligned}
\Psi_{j}(\vec{q}) & =\sqrt{I_{s(j)}} \frac{\mathcal{F}\left\{\psi_{j}\right\}}{\left|\mathcal{F}\left\{\psi_{j}\right\}\right|} \\
\psi_{j}^{\prime}(\vec{r}) & =\mathcal{F}^{-1}\left\{\Psi_{j}\right\}
\end{aligned}
$$

3. update object function

$$
O_{j+1}(\vec{r})=O_{j}(\vec{r})+\alpha \frac{P_{j}^{*}\left(\vec{r}-\vec{R}_{s(j)}\right)\left(\psi_{j}^{\prime}(\vec{r})-\psi_{j}(\vec{r})\right)}{\max \left\{\left|P_{j}\left(\vec{r}-\vec{R}_{s(j)}\right)\right|^{2}\right\}}
$$

4. update probe function

$$
P_{j+1}(\vec{r})=P_{j}(\vec{r})+\beta \frac{O_{j}^{*}\left(\vec{r}-\vec{R}_{s(j)}\right)\left(\psi_{j}^{\prime}(\vec{r})-\psi_{j}(\vec{r})\right)}{\max \left\{\left|O_{j}\left(\vec{r}-\vec{R}_{s(j)}\right)\right|^{2}\right\}}
$$

whereas $I_{j}$ denotes the measured intensity at each position $j,{ }^{*}$, denotes complex conjugation and $\mathcal{F}\{\}$ and $\mathcal{F}^{-1}\{\}$ symbolize the Fourier transformation and its inverse, respectively. $\alpha$ and $\beta$ determine the step size for the updates of the object and the probe. Typical values are $0<\alpha, \beta \leq 1$. In this thesis, good results have been obtained by choosing $\alpha=\beta=0.5$. The best choice, however, may be problem dependent, i.e. the step sizes have to be chosen for each dataset independently.

The convergence of the algorithm is usually monitored by an error metric such as

$$
E_{\Psi}=\frac{\sum_{j} \sum_{\vec{q}}\left|\sqrt{I_{j}(\vec{q})}-\right| \Psi_{j}(\vec{q})||^{2}}{\sum_{j} \sum_{\vec{q}} I_{j}(\vec{q})} .
$$

One important difference to the 'DM' algorithm is the probe update at each position. In case of the DM algorithm 294, the probe update appears to be practically carried out only once after cycling through all positions. For this reason, the ePIE update of the object may often be more advantageous than the update of the DM ptychographic algorithm. For instance, it is known that the convergence rate of the ePIE outperforms the DM algorithm [183]. One important advantage of the DM ptychographic procedure to update the probe only once in a while is that the application of the modulus constraint can be parallelised yielding much faster cycling times. At present, the pros and cons of both algorithms have not been analysed in a mathematically rigorous manner. From experience, the results using 'ePIE' are often significantly better compared to results that have been obtained with the DM version using the same number of iterations. However, for certain diffraction data such as biological specimens the results may remain comparable and a fast algorithm is obviously preferred. Giewekemeyer and colleagues also published one case, where not a single out of both algorithm was able to yield satisfying results. Surprisingly, switching between both algorithms could be used to obtain good reconstructions $[96]$. 


\subsubsection{Tomography}

The mathematical basis to reconstruct a $3 \mathrm{~d}$ object from a set of $2 \mathrm{~d}$ projections was presented first of all by Johann Radon 245 . Nowadays the theory of tomographic imaging can be found in many text books such as 33,151 . This section is dedicated to recapitulate the mathematical concepts following 'Principles of Computerized Tomographic Imaging' by A.C. Kak and M. Slaney 151.

\subsubsection{The Fourier Slice Theorem}

The key to understand the tomographic principle is the Fourier Slice Theorem (FST). Briefly stated, a two-dimensional object can be reconstructed from an angular series of one-dimensional projections. Each projection carries a part of the full information of the objects' Fourier representation. The object can then be reconstructed by using the projections to fill up the objects Fourier domain and its inversion.

The mathematical formulation of the FST requires the definition of the Radon transform $P_{\theta}(t)$,

$$
P_{\theta}(t)=\iint_{\mathbb{R}^{2}} f(x, y) \delta(x \cos \theta+y \sin \theta-t) d x d y,
$$

which is a line integral through an object at an angle $\theta$. As a function of $t, P_{\theta}(t)$ yields the parallel projection of the function $f$ at an angle $\theta=$ const. The FST is obtained by taking the one-dimensional Fourier transform of eqn. (1.101)

$$
\begin{aligned}
S_{\theta}(\nu) \equiv \mathcal{F}\left\{P_{\theta}(t)\right\}(\nu) & =\int_{\mathbb{R}} P_{\theta}(t) \exp (-i 2 \pi \nu t) d t \\
& =\iint_{\mathbb{R}^{2}} f(x, y) \exp (-i 2 \pi \nu(x \cos \theta+y \sin \theta)) d x d y
\end{aligned}
$$

That is,

$$
\mathcal{F}\left\{P_{\theta}(t)\right\}(\nu)=\mathcal{F}\{f(x, y)\}(\nu \cos \theta, \nu \sin \theta)
$$

The FST states that the Fourier transform of a projection $P_{\theta}(t)$ taken of an object $f(x, y)$ at an angle $\theta$ is equal to the slice at an angle $\theta$ of the objects' Fourier representation. A sketch of the FST of eqn. (1.102) is presented in figure 1.14 .

\subsubsection{Filtered Back Projection Algorithm}

The FST suggests a formal reconstruction of the object by filling up its Fourier domain with radial lines and subsequently, carrying out the inversion. The following mathematical expression for $f(x, y)$ can be obtained by making a coordinate 


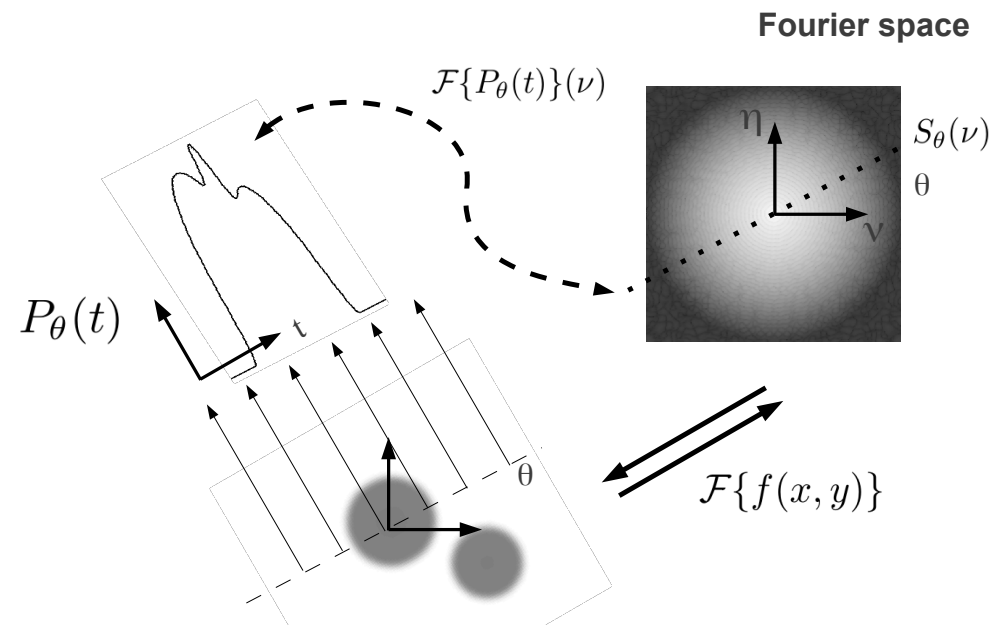

Real space

Figure 1.14: The image sketches the Fourier Slice Theorem of eqn. (1.102). The two spheres $f(x, y)$ form the projection $P_{\theta}(t)$ at an angle $\theta$. A slice $S_{\theta}(\nu)$ of the Fourier transform $\mathcal{F}\{f(x, y)\}$ is equal to the Fourier transform of the Projection, $\mathcal{F}\left\{P_{\theta}(t)\right\}(\nu)$.

transform to polar coordinates within the inverse Fourier transform of the Fourier representation of $f(x, y)$,

$$
\begin{aligned}
\Rightarrow f(x, y) & =\int_{0}^{\pi} Q_{\theta}(x \cos \theta+y \sin \theta) d \theta \\
\text { where } \quad Q_{\theta}(t) & =\int_{0}^{\nu_{c}} S_{\theta}(\nu)|\nu| \exp (i 2 \pi \nu t) d \nu .
\end{aligned}
$$

The 'filtering' becomes apparent in eqn. (1.104): the wedge filter $|\nu|$ (cut-off frequency $\nu_{c}$ ) is applied to the radial line $S_{\theta}(\nu)$ before transforming back to the object space. The polar coordinates are inherent in the representation of the object by eqn. (1.103). The role of $Q_{\theta}(t)$ is best understood if it is regarded as an almost inverse operation of the parallel projection $P_{\theta}(t)$. In contrast to the projection, $Q_{\theta}(t)$ carries the same value for all object points along the straight line $t=x \cos \theta+$ $y \sin \theta$ which is referred to 'back projection' or 'back smearing'. The diversity along the line $t=x \cos \theta+y \sin \theta$ is obtained by summing up the different information of sufficiently many angles. Next, a full three-dimensional reconstruction of an object can be obtained by applying the filtered back projection algorithm separately to projections of single, successive planes that lie perpendicular to the axis of rotation. This section is closed by making a remark about the dependence of the resolution $\Delta$ of the tomogram on the number of projections $N$ and the size $d$ of the object in the direction of the beam. The 'Crowther' criterion yields 53,178 


$$
\Delta=\frac{\pi d}{N}
$$

Con-

sequently, thicker objects require more projections to obtain a comparable resolution. 



\section{Bacterial Samples}

The sample systems that are treated of in this section, namely Deinococcus spp. ${ }^{8}$ and Bacillus subtilis spp., are well studied microorganisms. Much of the presented knowledge can be found in the general textbook literature 180 . In case of Bacillus subtilis, there is also ample literature in the form of review articles 28, 119. In addition, this section is also dedicated to the techniques that have been applied for sample preparations, which includes cell culturing, fixation and embedding for TEM imaging and vitrification and lyophilisation for X-ray imaging experiments.

\subsection{Deinococcus radiodurans}

The bacterium Deinococcus radiodurans ${ }^{9}$, formerly known as Micrococcus radiodurans, has been isolated for the first time from canned meat by intensive $\gamma$ radiation $|7|$. The bacterium is nonpathogenic, nonmotile, red-pigmented, Grampositive and well-known due to its outstanding resistance to ionizing-radiation and oxidative stress $77,51,55,56,164,169,180,184,277$. For instance, D. radiodurans $\mathrm{R} 1$ reaches a $D_{10}$ value of $10 \mathrm{kGy}|51|$, i.e. $10 \%$ of the cells of a culture can be activated to growth after irradiation. Moreover, the bacterium is able to actively grow under chronic irradiation of $50 \mathrm{~Gy} / \mathrm{h}|56|$. Along the line of extreme resistance, $D$. radiodurans has been shown to be resistant to desiccation on the scale of years and UV-radiation 56,277 .

At present, there is no evidence for a single special mechanism that could explain the unparalleled survival rates of $D$. radiodurans. Explanations include suggestions of special repair mechanisms for DNA double strand breaks [51], and it was shown that a high manganese content within the cells facilitates resistance to ionizing radiation [55]. As a hypothesis, it has been proposed that protein oxidation caused by reactive oxygen species such as hydroxyl radicals $\left(\mathrm{OH}^{\bullet}\right)$ that arise due to radiolysis of $\mathrm{H}_{2} \mathrm{O}$ is signficantly reduced by the manganese content $[56$. A somewhat controversial, discussion has been raised by indications that a toroidal shape of the nucleoid plays a role for its resistance to ionizing radiation [169] (cf. 202, 203 342 for discussion). A TEM image of the toroidal shape of the DNA is presented in figure $2.16(\mathrm{~b})$. Interestingly, the condensation of the genome may indeed be an important factor $\mid 342]$. The compaction of DNA likely helps to conserve it due to e.g. a decrease of diffusion of DNA fragments, a protection from free radicals and by preventing double strand breaks caused by proximal single strand breaks [277].

Deinococcus spp. have been isolated from extreme environments such as hot springs (D. geothermalis), Antarctic soil (D. frigens) or the Gobi desert (D. gobiensis) (cf. $|277|)$. The complete genome of $D$. radiodurans (strain $\mathrm{R} 1$ ) consists of about $3.3 \cdot 10^{6}$ base pairs: two chromosomes of $2,648,638$ and 412,348 base pairs, a megaplasmid of 177,466 base pairs and a small plasmid of 45,704 base pairs 326 . The cells are polyploid meaning they contain multiple copies of the

8 spp. = species pluralis

9 deinos (Greek) means 'strange' or 'unusual' whereas radiatio and durans (Latin) denote 'radiation' and 'endure', respectively 26 . 
genome (2-10) $[51,66,117,118,277$. Phylogenetic analyses indicate a close relation of Deinococceae to the Thermus species and that they are part of a common clade (phylum) $72,126,282$.

In contrast to other enduring microorganisms such as Bacillus spp., D. radiodurans is nonsporulating. It is typically grown under strict aerobic conditions at $30-37^{\circ} \mathrm{C}$ in rich TGY medium $(0.5 \%$ tryptone, $0.1 \%$ glucose, $0.15 \%$ yeast extract) 277 but other media for high cell density production are known as well [120]. The bacterial cells occur as single cocci and diplococci but tetrads are often abundant (cf. Fig. 2.15, 2.16). They exhibit a typical spherical shape ${ }^{10}$, which has a diameter of $1.5-3 \mu \mathrm{m} \mid 26]$.

The multilayered cell envelope of $D$. radiodurans is untypical for Gram-positive bacteria. To date, there have been identified five to six layers: (i) the cytoplasmic membrane, (ii) the rigid peptidoglycan-containing holey layer, (iii) the compartmentalized layer, (iv) an outer membrane, (v) a distinct electrolucent zone, and (vi) the S-layer which contains hexagonally packed subunits [184,277]. There are many studies addressing the fine structure of $D$. radiodurans by using TEM and thin slices of stained and resin-embedded cellular material but also cryo-electron microscopy of vitreous sections and cryo-electron tomography studies have been carried out $48,75,76]$. In these cryo-electron images, the contrast between the DNA of the nucleoid and the surrounding cytoplasma including ribosomes is small but sufficient to analyse the local ordering. That is, the fine-structure exhibits dominant or significant Fourier components down to the resolution of the imaging apparatus. In particular, the results of Eltsov and Dubochet indicate that (i) there is no local ordering (in terms of Fourier components) in the bacterial nucleoid of exponentially growing cells, (ii) there appears ordering corresponding to a realspace size of $4-6 \mathrm{~nm}$ in stationary-phase cells and (iii) the ordering condenses to a real-space size of $4 \mathrm{~nm}$ in long-stationary-phase cells $\mid 75$.

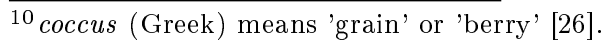




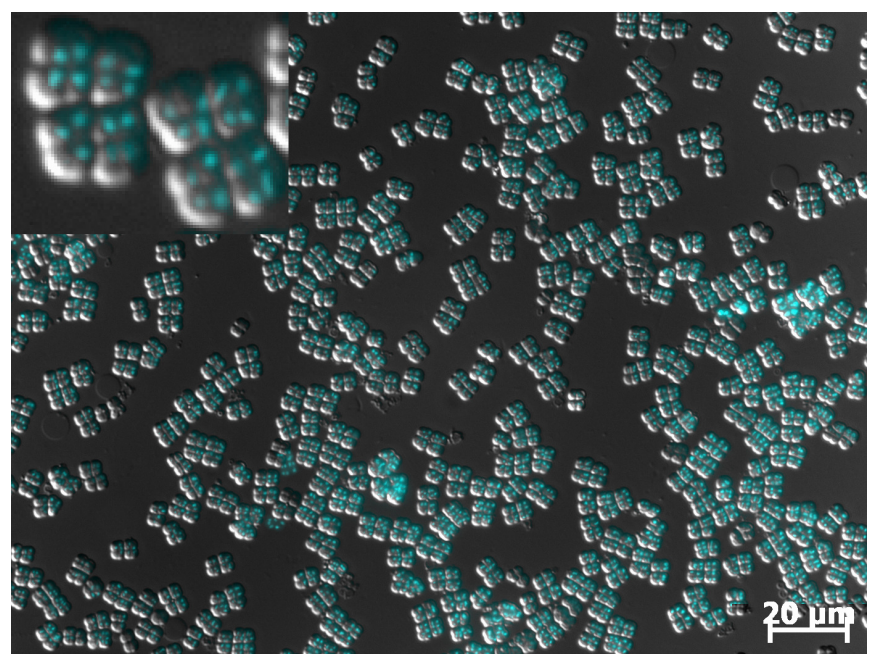

(a)

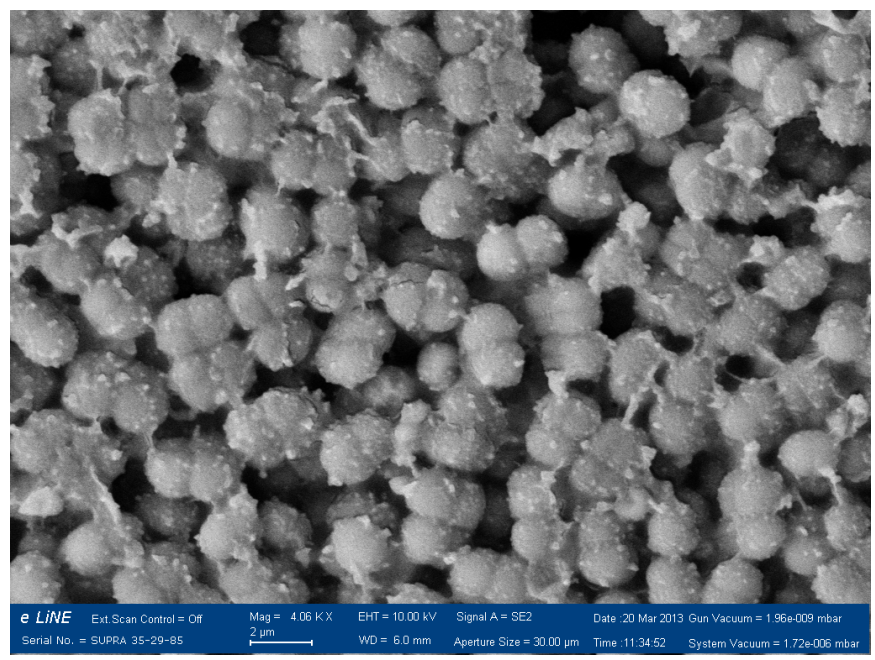

(b)

Figure 2.15: (a) Overlay of optical light micrograph (DIC, 63x) and fluorescence signal of DAPI (4',6-Diamidino-2-phenylindole dihydrochloride; turquoise) stained DNA showing cells of $D$. radiodurans $\mathrm{R} 1$ in buffered solution. The inset shows a zooming-in on specific cells of tetrads. The two images have been taken in the laboratory within a short time sequence shortly after each other. The contrast and color has been altered for optimum visibility. (b) SEM image of air-dried D. radiodurans cells, which have been sputtered with $3 \mathrm{~nm} \mathrm{Ti}$ and $15 \mathrm{~nm}$ Au. Tetrads of cells can be clearly seen. A cell thickness of about $1.5 \mu \mathrm{m}$ is inferred. 

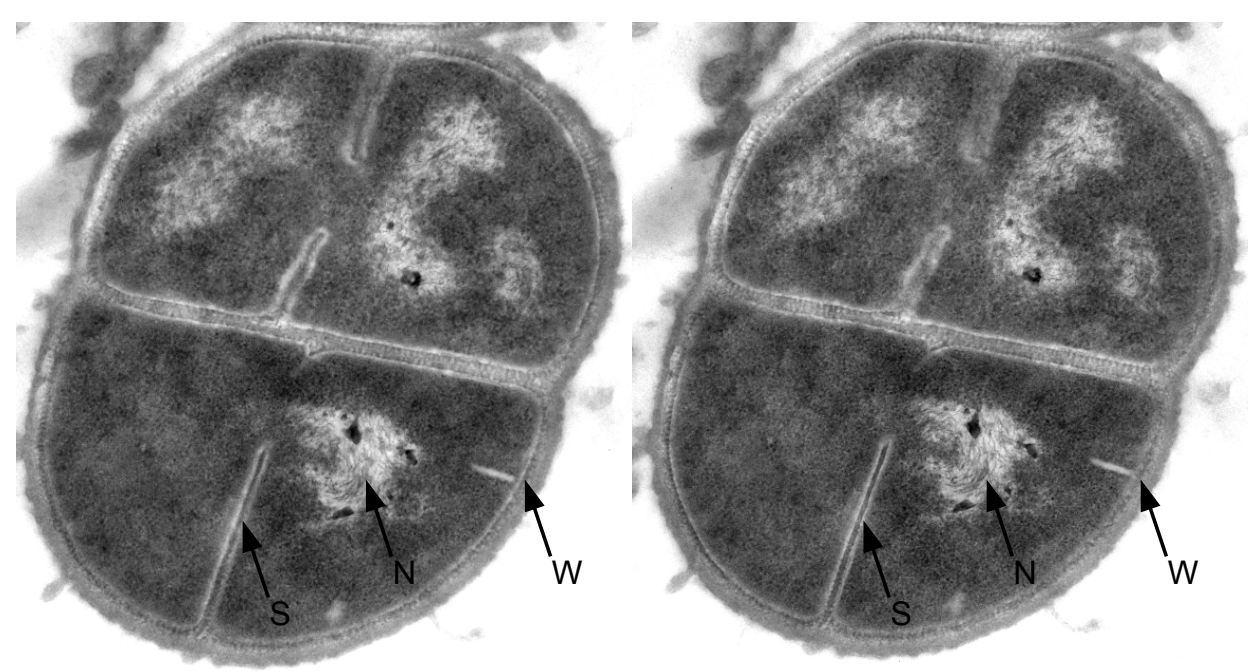

(a)

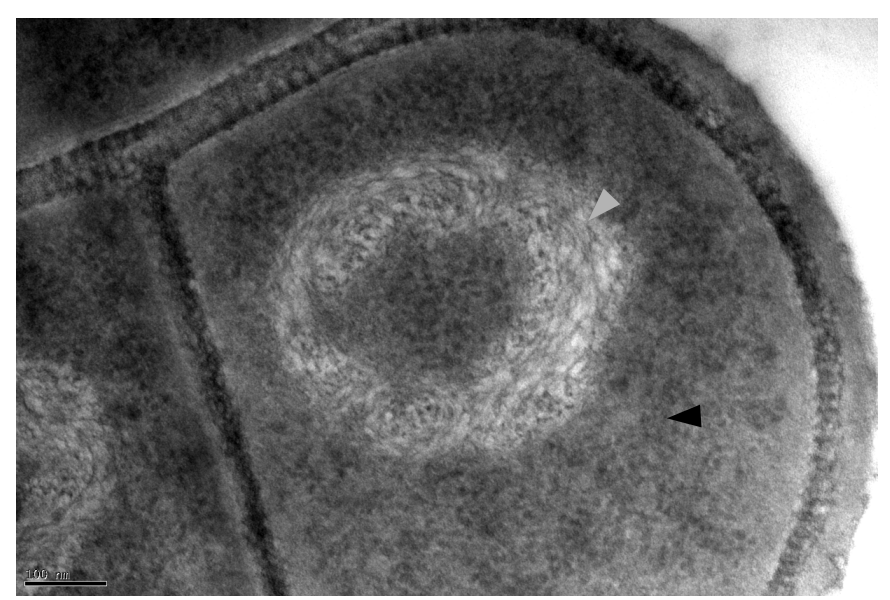

(b)

Figure 2.16: (a) Two TEM images of a thin slice of D. radiodurans cells being stained with $\mathrm{OsO}_{4}$ (Osmium tetroxide) and $\mathrm{C}_{4} \mathrm{H}_{6} \mathrm{O}_{6} \mathrm{U}$ (Uranyl acetate) which have been taken with an angular difference of about $20^{\circ}$, i.e. stereo geometry. Indicated are incomplete septa (S) in the cytoplasma, the cell wall (W) and the DNA of the bacterial nucleoid (N). (b) shows the bacterial nucleoid of D. radiodurans at high magnification. In this section the nucleoid (grey arrowhead) appears as a ring-like structure with a proteinaceous core. The outer diameter is about $350-430 \mathrm{~nm}$, whereas the core has a diameter of about $180-230 \mathrm{~nm}$. Ribosomes appear as dark, grainy structure within the cell (e.g. black arrowhead). 


\subsection{Bacillus subtilis \& Bacillus thuringiensis}

The genus Bacillus is known since Ferdinand Cohn's work on Bacillus subtilis (1872) $[119]^{11}$. The bacteria of this genus have become model organisms for microbial life, industrial applications such as biological insecticides and biological warfare 119, 132, 194,281. They are Gram-positive, rod-shaped, grow under aerobic conditions and are well-known for their ability to transform into the methabolically dormant state of an endospore $[79,119,180,193,213,214$. In this dormant state, the genetic information of the organism is very effectively stored due to an increased resistance to e.g. wet and dry heat, desiccation, ionizing radiation such as UV and $\gamma$ and chemical agents 213. Reports of longevity include a sample of B. anthracis that was originally stored by L. Pasteur in 1888 and which showed active growth 68 years later but even longer resting times are possible 158 . Even, reports such as 'revival' of Bacillus spores from insect inclusions of amber that are millions of years old appear $\mid 34]^{12}$. Cell culturing from spores of very old samples remains controversial.

The change of the vegetative cycle of the cell towards sporulation is induced when one or more nutrient sources such as carbon, nitrogen or phosphorus become depleted [119, 180,193]. Typically, endospores can be observed in a cell culture that is in the transition state between exponential growth and stationary phase. The process can also be controlled by transferring a cell culture to a medium with negligible nutrients or by chemical treatments $[119$. The process from vegetative growth to a mature endospore includes the following stages: (i) building of the forespore compartment, that is separated from the mother cell by the septum by asymmetric cell division, (ii) engulfment of the forespore within the mother cell, (iii) surrounding of the free forespore by two distinct layers of cytoplasmic membrane, (iv) forming of the cell wall and cortex, (v) building of the spore coat, (vi) maturing of the endospore (full heat resistance) and (vii) lysis of the mother cell $[119$. The full process ending in a free endospore takes about $7-8$ hours in case of $B$. subtilis $[180,229$. Vegetative growth is continued after germination of the endospore $205,230,272$.

The morphology of the endospore is as follows: (i) the core contains the chromosome and is enclosed by the cytomembrane (inner forespore membrane), (ii) the cortex, (iii) outer forespore membrane, (iv) basement layer, (v) inner coat, (vi) outer coat, (vii) crust and, depending on the subspecies, (viii) an exosporium including possibly appendages [180,193]. Endospores exhibit several remarkable physical properties. Firstly, the core is comparably dehydrated (by a factor of about 0.4 ) with respect to the outer layers, which is one reason for the heat resistance of endospores $\left.{ }^{13} \mid 19,69\right]$. The nucleoid of $B$. subtilis is transformed into a condensed state in the core during sporulation [69 where the DNA becomes toroidal 233. Occasionally, the state of the DNA is also referred to as crys-

\footnotetext{
${ }^{11}$ According to http://en.wikipedia.org/wiki/Bacillus_subtilis (15/04/2014) Bacillus subtilis was given the name Vibrio subtilis by Christian Gottfried Ehrenberg in 1835.

${ }^{12}$ Interestingly, the report of Cano and Borucki in 1995 was published shortly after the science fiction movie Jurassic Park (1993), which is based on the Novel by Michael Crichton.

${ }^{13}$ Heat resistance may also be increased due to rotationally immobilized proteins in the core 283 .
} 
talline 68 . It should also be noted that the core is highly calcified 69 . Next, the structure of particular spore elements can be highly ordered. For instance, a socalled honeycomb crystalline layer with a period of about $9 \mathrm{~nm}$ and $\sim 5-6 \mathrm{~nm}$ holes or pits seems to be present in the coat of many Bacillus species [231,232]. Finally, it became only recently understood that the endospore morphology changes drastically due to an increase or decrease of relative humidity of its environment 68,325 . The endospore is able to absorb water and release it reversibly 232 . In particular, it was shown by Westphal et al. 325 that endospores undergo swelling when exposed to an increase in relative humidity. They could identify two different swelling time scales $\left(t_{1}<50 \mathrm{~s}\right.$ and $\left.t_{2} \approx 8 \mathrm{~min}\right)$, that may be related to diffusion times of water into coat \& cortex and core, respectively.

Bacteria of the type Bacillus thuringiensis have been studied intensively for their pathogenecity to specific insects $[9: 263$. The pathogenecity arises due to the ability of the bacteria to produce parasporal crystalline inclusions of pesticidal toxins, socalled Cry- and Cyt- proteins or protoxins [52], during the stationary phase [263. These protein crystals consist of about $10^{6}$ protein molecules, which account for $20-30 \%$ of the cell mass (without water) 2,16 . The morphology of the crystals depends on the bacterial strain. For instance, bipyramidal and cuboid shapes can be found in B. thuringiensis subsp. kurstaki, whereas also ovoid structures can be found in B. thuringiensis subsp. israelensis [9]. The toxicity of the proteins is fully developed in the midgut of insect larvae due to a reaction with local proteases. In the solubilized, toxic state the proteins bind to apical cell membranes of the insect midgut and create ion channels or pores, which in turn leads to lysis of the cells 92,263 and death of the insect. Structure analyses of the toxins on the scale of atomic resolution have been carried out with X-ray diffractometers (cf. [170]).

B. thuringiensis 'Berliner 1915', which was studied in this thesis, is pathogen to lepidoperan larvae 263. Its crystals appear in a tetragonal form 116. Purification of the crystals can be obtained by different centrifugation techniques $[8,36,339]$. 

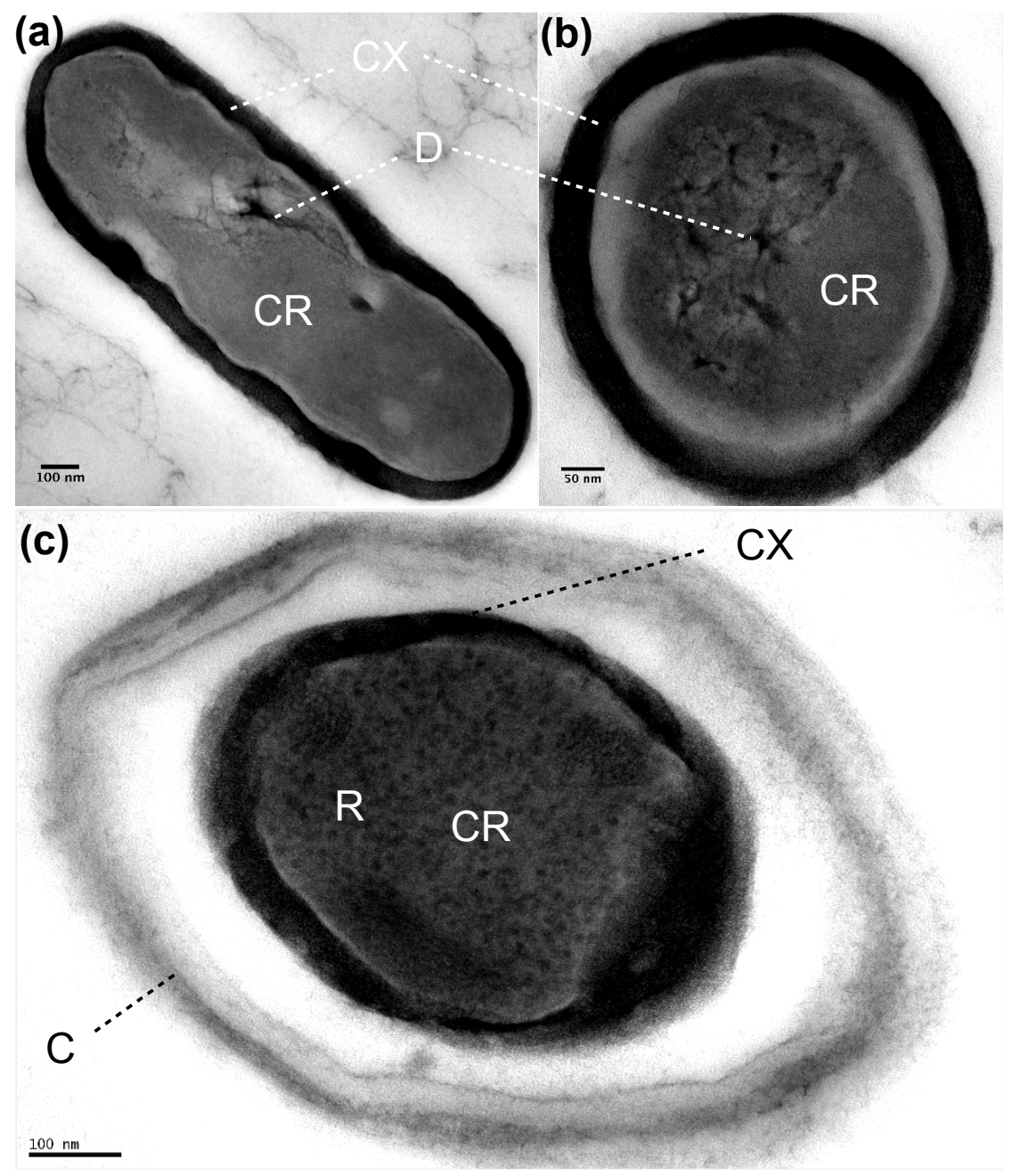

Figure 2.17: TEM images of endospores of Bacillus subtilis. (a), (b) show longitudinal and transversal sections, respectively. The cortex (CX) around the core (CR) is visible but the coat appears to be missing. The condensed material (D) in the core is supposedly DNA in an artificial aggregated state. The extent of major and minor axes of the endospore in (a) are about $1600 \mathrm{~nm}$ and $\sim 600 \mathrm{~nm}$, respectively. The diameter in (b) is in the range of $500-560 \mathrm{~nm}$. (c) presents a TEM image with an intact coat $(\mathrm{C})$. Further details are cortex $(\mathrm{CX})$ and the core $(\mathrm{CR})$ with ribosomes (R). The major and minor axes of the core including cortex extent to about $630 \mathrm{~nm}$ and $460 \mathrm{~nm}$, respectively. Taking also the coat into account yields about $1030 \mathrm{~nm}$ along the major axis and approx. $700 \mathrm{~nm}$ along the minor axis. 

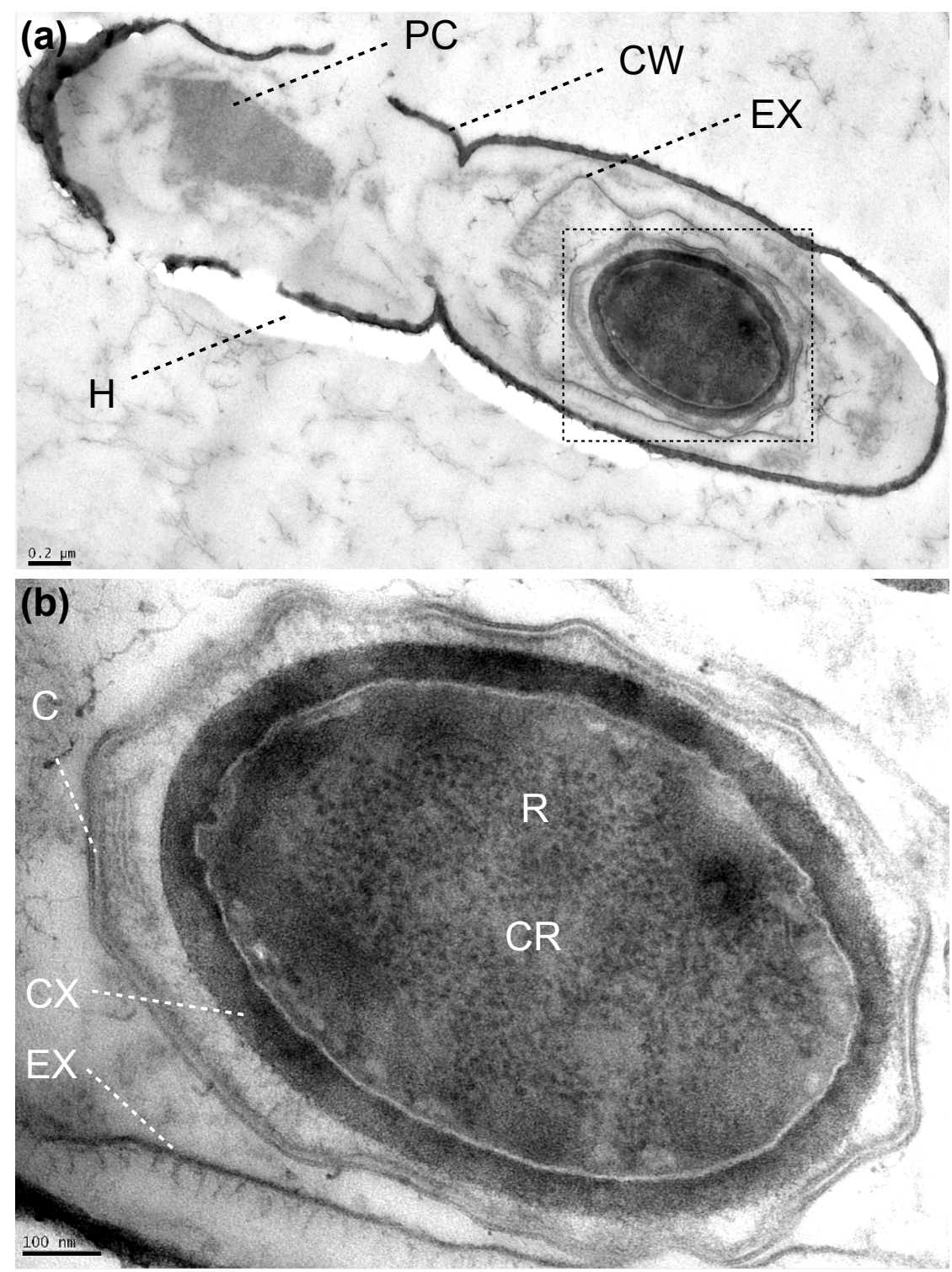

Figure 2.18: (a) shows a TEM image of two lysing Bacillus thuringiensis cells. The cell walls $(\mathrm{CW})$ are already fragmented. One cell contains the typical protein crystal (PC), whereas the other cell is occupied by an endospore (dashed frame), that is surrounded by the exosporium (EX). Holes $(\mathrm{H})$ in the TEM slice are visible. The protein crystal has dimensions of about $880 \mathrm{~nm}$ and $440 \mathrm{~nm}$ along the major and minor axes, respectively. The extent of the endospore including the coat is about $1190 \mathrm{~nm}$ along the major axis and $760 \mathrm{~nm}$ along the minor axis. (b) is a close-up of the endospore as indicated by a dashed frame in (a), revealing details of the exosporium $(\mathrm{EX})$, coat $(\mathrm{C})$, cortex $(\mathrm{CX})$ and the core (CR). This section of the core contains mainly ribosomes $(\mathrm{R})$. 


\subsection{Sample Preparations}

\subsubsection{Cell Culture of D. radiodurans}

The bacteria D. radiodurans R1 (DSM no. 20539, Leibniz-Institute DSMZ-German Collection of Microorganisms and Cell Cultures $\mathrm{GmbH}$ ) are cultivated on solid agar plates using Corynebacterium Agar (DSMZ medium 53, Tab. B.1, p. 192). Pure cell cultures from the DSMZ contain a dried cell pellet that needs to be dissolved in liquid medium. After an incubation of $30 \mathrm{~min}$ at room temperature, the cell suspension is homogeneously distributed on agar plates in amounts of about $200 \mu$ l. Cells have been incubated under slightly varying conditions such as different temperatures $\left(30^{\circ} \mathrm{C}-37^{\circ} \mathrm{C}\right)$. Incubation at lower temperatures slows down desiccation of the agar plates but higher temperatures may yield faster growth rates. The DSMZ recommends incubation at $30^{\circ} \mathrm{C} \mathrm{(cf.} \mathrm{Fig.} \mathrm{2.19).} \mathrm{Typically,} \mathrm{in-}$ cubation is stopped as soon as the whole surface of the plate is covered visibly with a thin layer of bacteria, which happens to be after $24-48 \mathrm{~h}$. Longer incubation times facilitate forming of a thick, sticky bacterial mat, which should be avoided. In order to reduce the ratio of dead to viable cells, a repetition of the inoculation and incubation step can be carried out by using cells from the actively growing culture. After incubation, the cells are gently washed from the culture plate by using $1-1.5 \mathrm{ml}$ phosphate buffered saline (PBS; Dulbecco's Phosphate Buffered Saline, Sigma-Aldrich). Next, the cell suspension is washed 1-2 times with PBS in $1.5 \mathrm{ml}$ tubes and by using comparably gentle centrifugation at $0.6 \mathrm{~g}$ for $60 \mathrm{~s}$. At the bottom of the tube a layer of about $50-80 \mu \mathrm{l}$ of red or pinkish cell pellet is obtained. Short time storage (over night) at $4-8{ }^{\circ} \mathrm{C}$ in a fridge follows before further processing of the cell material. 
(a)

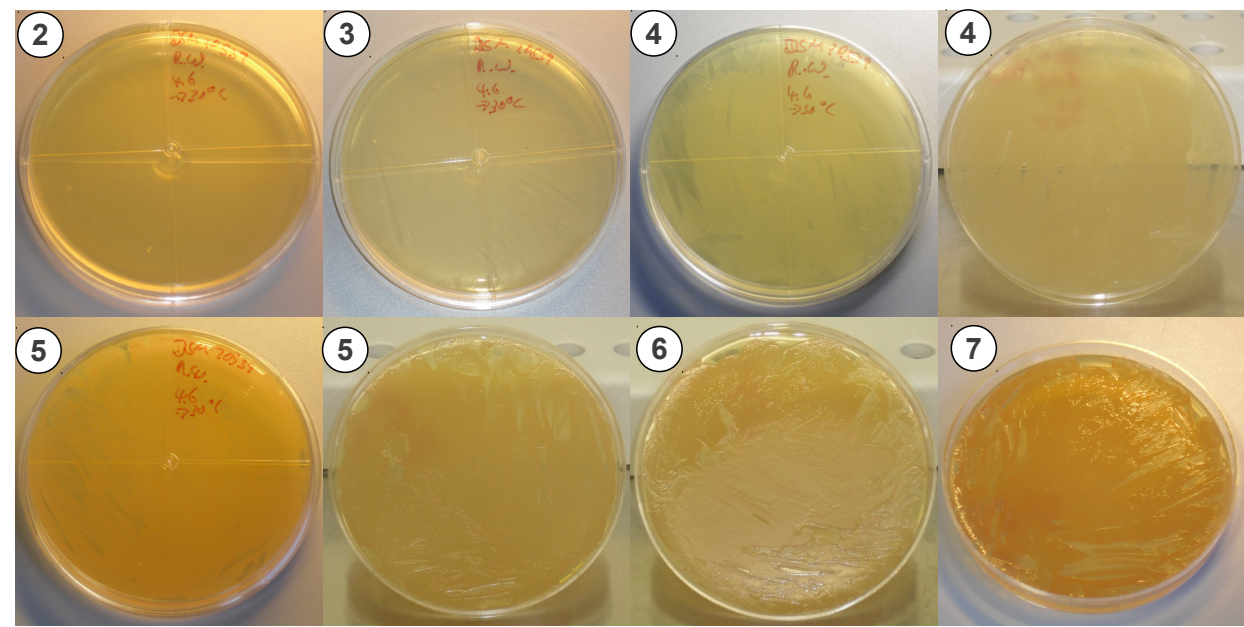

(b)

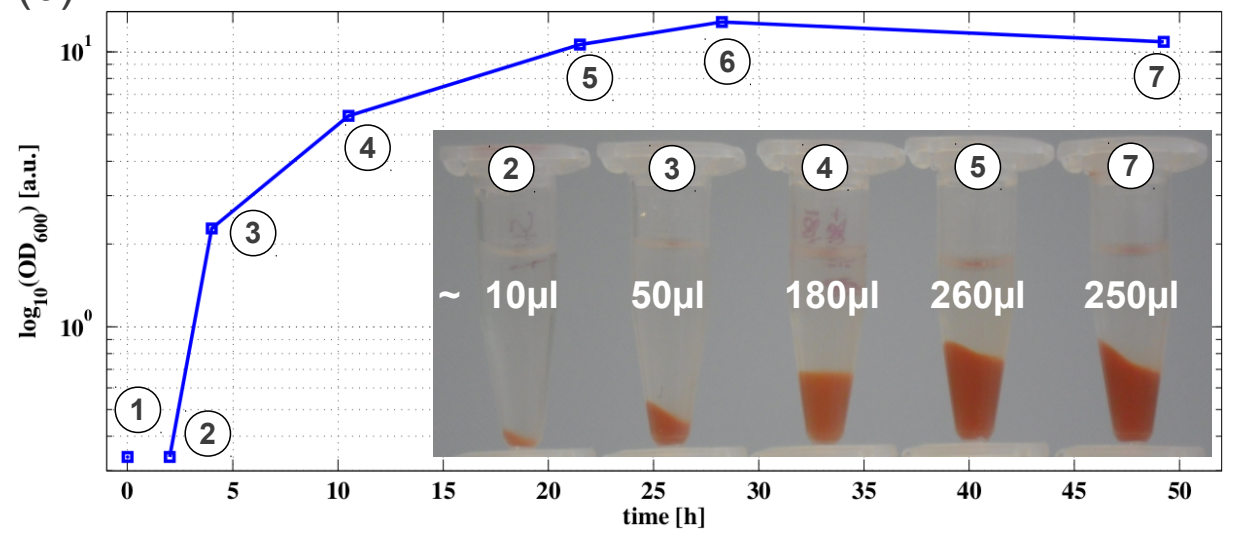

Figure 2.19: Growth of D. radiodurans cells on solid agar medium M53 at $T=$ $30^{\circ} \mathrm{C}$. Approx. $100 \mu \mathrm{l}$ of cells of an actively growing culture was incubated for $10 \mathrm{~min}$ in $1 \mathrm{ml}$ liquid M53 at room temperature. Thereafter, this cell suspensions was distributed in amounts of $100 \mu \mathrm{l}$ on fresh solid media plates, i.e. each agar plate was homogeneously covered with $10 \mu \mathrm{l}$ of cells. Next, the growth was monitored and quantified by inspecting single agar plates at different time intervals. (a) presents photographs taken on the agar plates before harvesting. The photos show the culture through the top of the petri-dish $(2,3)$, from below $(6,7)$ and from both sides $(4,5)$. Note the formation of a thick film at (4). (b) shows optical density measurements (Spectrophotometer Nano Drop ND-1000, Peqlab, Germany) of the harvested cell cultures (suspended in PBS) of (a) at different times (2-7). Point (1) indicates the begin of the incubation but it does not represent a true measurement; its value is assumed to be the same as (2) due to no measurable increase in cells. The inset indicates corresponding cell yields. 


\subsubsection{Cell Culture of B. subtilis \& B. thuringiensis}

The growth medium used for both Bacillus subspecies is Lysogeny broth (LB) (Tab. B.2, p. 192). It is a nutrient rich broth that can be used for many bacterial strains. Firstly, pure cell colonies of B. subtilis 168 ('Ehrenberg 1835') 'Cohn 1872' (DSM no. 23778, Leibniz-Institute DSMZ-German Collection of Microorganisms and Cell Cultures GmbH) ${ }^{14}$ and B. thuringiensis 1564 'Berliner 1915' (DSM no. $350)^{15}$ are incubated for 1 day in a shaking incubator (Innova 42, Eppendorf) at $30^{\circ}(200 \mathrm{rmp})$ in $20 \mathrm{ml} \mathrm{LB}$ medium in a $100 \mathrm{ml}$ Erlenmeyer flask defining the preculture. In case of B. subtilis, inoculation of $100 \mathrm{ml} \mathrm{LB}$ medium in a $500 \mathrm{ml}$ Erlenmeyer flask with about $10 \mu \mathrm{l}$ cell suspension is initiated on day 2. A transfer of $B$. thuringiensis to the same growth conditions has a negative influence on the spore and protein crystal yields. Best results have been obtained by keeping the combination of $20 \mathrm{ml}$ medium in a $100 \mathrm{ml}$ volume flask. Hence, on day 2 about $10 \mu \mathrm{l}$ cell suspension of $B$. thuringiensis is transferred to $20 \mathrm{ml}$ of fresh LB medium in a $100 \mathrm{ml}$ Erlenmeyer flask. The cell growth has been monitored via optical light microscopy (Fig. 2.20) and optical density measurements (Fig. 2.21), which suggests that sporulation starts already after approx. $9 \mathrm{~h}$ of incubation (the onset of the log-phase). However, in order to obtain significant yields of endosporulation both cell cultures are simply incubated for 6 days. Finally, endospores can be harvested on day 7 .

The material is purified by successive centrifugation and washing steps. Firstly, remnant medium is removed from the endospores suspension by $10 \mathrm{~min}$ of centrifugation at $2000-4000 \mathrm{~g}$, favourably at $4{ }^{\circ} \mathrm{C}$. Next, one or more washing steps with pure water are carried out using $1.5 \mathrm{ml}$ tubes and centrifugation for $10 \mathrm{~min}$ at $5000 \mathrm{~g}$. In case of B. subtilis, a pellet of two layers of material already becomes visible after this secondary centrifugation procedure. The upper, dark layer consists of endospores, whereas the lower, brighter layer is formed by remnant cell material. Consequently, the bright layer is discarded. After 6 days of incubation, there are significantly less free endospores in the culture of B. thuringiensis than in the one of B. subtilis. Hence, only one layer of mixed material remains in the tube. After these washing steps, the material can be stored for at least one day at $4-8{ }^{\circ} \mathrm{C}$ in a fridge. Long term storage is not advisable as spontaneous germination can be expected 119 . Of course, the purity of the B. subtilis endospore material undergoing long term storage can be controlled by centrifugation being followed by removing the bright layer.

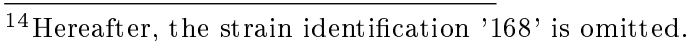

${ }^{15}$ Henceforth, the strain identification '1564' is omitted.
} 


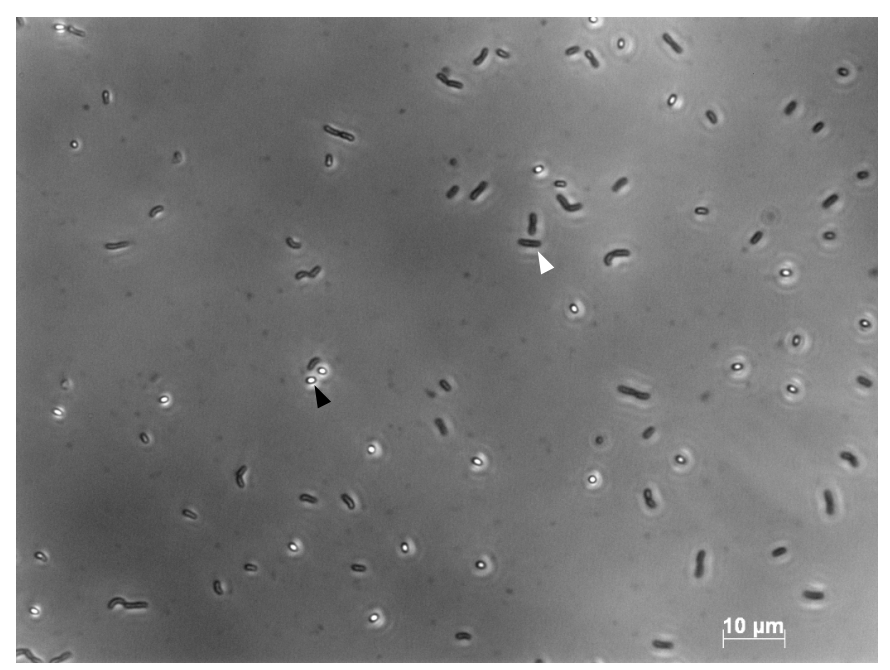

(a)

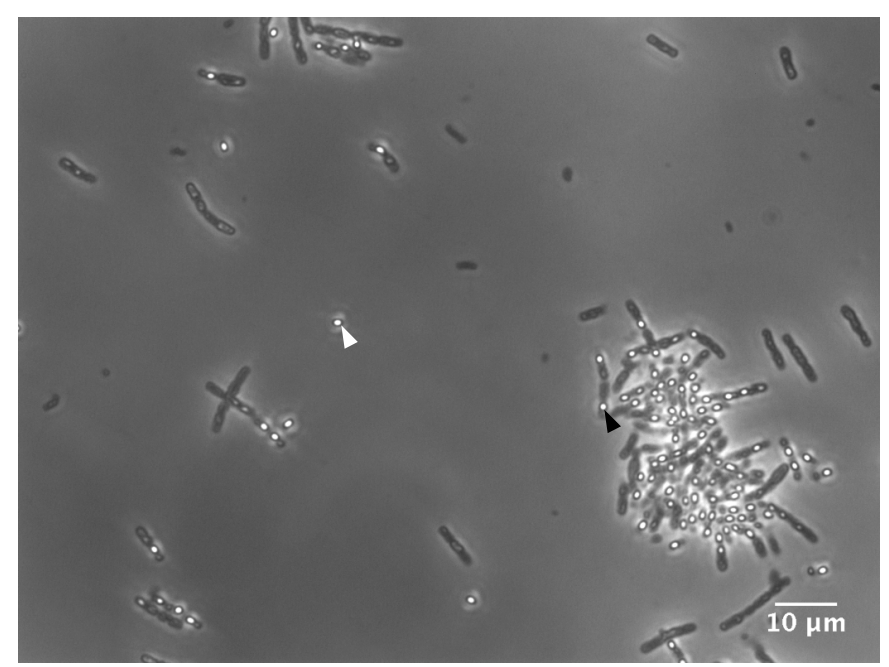

(b)

Figure 2.20: (a) cells of Bacillus subtilis in solution can be seen on the phase contrast optical light micrograph (40x, NA=0.75, Neofluar Zeiss; Zeiss Axioskop 40) as indicated by the white arrow head. Isolated endospores, which appear as bright spots due to their high refractive index, are typical for this cell culture after several days of incubation (e.g. black arrow head). (b) The image shows an optical light micrograph (phase contrast) of cells of Bacillus thuringiensis in solution after several days of incubation. Many of the cells have formed intracellular endospores (e.g. black arrow head). In addition, a few isolated endospores are visible as well (e.g. white arrow head). 


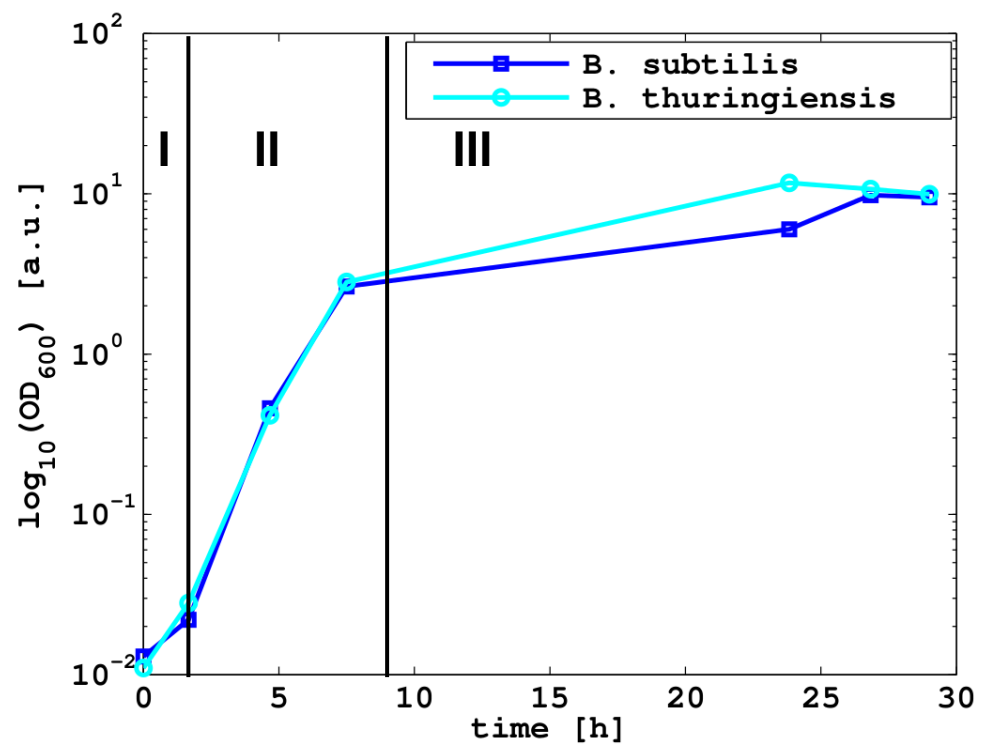

Figure 2.21: Optical density measurements (Biochrom WPA, Biochrom, UK) of Bacillus subtilis and Bacillus thuringiensis cell cultures indicate different growth stages: (I) lag-phase, (II) exponential growth and (III) stationary phase. Both cultures have been pre-incubated $24 \mathrm{~h}$ before measurements. At time zero growth was initiated in flasks with $100 \mathrm{ml}$ sterile LB medium. Incubation was carried out at $30^{\circ} \mathrm{C}$ using 200 RPM.

\subsubsection{Preparation of Ultra-thin Sections for Transmission Electron Mi- croscopy}

The preparation of ultra-thin sections (thickness approx. $80 \mathrm{~nm}$ ) of resin embedded, chemically fixed and stained samples is a standard procedure for electron microscopy [156. Here, a protocol from M. Hoppert is followed (see also 140 ). At first, the bacterial cells (in PBS) are chemically fixed with $2.5 \%(\mathrm{v} / \mathrm{v})$ glutaraldehyde. After an incubation of $90 \mathrm{~min}$ on ice, the material is washed at least once with PBS. Next, the buffer is almost entirely removed until the remnant material still allows to be resuspended. The cell material is then mixed with an equal amount of liquid 2\%(w/v) Bacto-agar (Agar Bacteriological, Oxoid LP0011, UK)/ PBS. The agar-PBS is kept in a liquid state at $50{ }^{\circ} \mathrm{C}$ before mixing it with the cells. Thereafter, the liquid is allowed to cool down and solidify. In the following step, the solidified agar/ cell suspension is cut with a razor blade into cubes with side length $1 \mathrm{~mm}$. The agar cubes are then incubated for $1-2 \mathrm{~h}$ in a $1 \%(\mathrm{w} / \mathrm{v})$ solution of osmium tetroxide. Afterwards, the aliquots are washed several times $(\geq 3)$ with pure water. Next, the samples are dehydrated via an ascending series of ethanol concentration followed by embedding with the resin LR White (Agar Scientific, UK;cf. Tab. C.1, p. 192). Single aliquots are then transferred into resin filled gelantine capsules. Finally, polymerisation is carried out by baking the 
capsules for at least $24 \mathrm{~h}$ at $50{ }^{\circ} \mathrm{C}$. Cutting ultra-thin sections from the resin-cell block requires appropriate pre-shaping by e.g. milling. The optimum shape is given by a pyramid with rectangular base and without the apex, i.e. a rectangular top (cf. Fig. 2.22). The top includes the block of bacterial material. Starting from the flat top, ultra-thin sections of the material along the normal axis of the top are obtained by cutting with a microtome (Ultracut E, Reichert-Jung). Only glass knives have been prepared and used in the context of this work. The ultra-thin sections are transferred onto TEM sample grids, which have been covered with a thin support film of formvar. Before TEM imaging, the sample sections are negatively stained by incubating them for $2 \mathrm{~min}$ on a droplet of $4 \%(\mathrm{w} / \mathrm{v})$ aqueous solution of uranyl acetate.

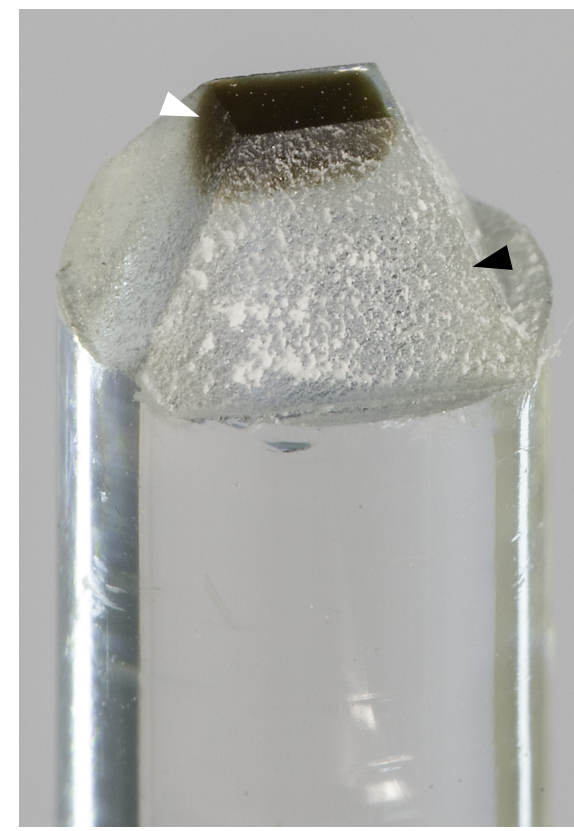

Figure 2.22: The image shows a resin embedded and chemically fixed sample of $D$. radiodurans cells. The trimmed top allows preparation of ultra-thin sections with a microtome. The white arrow indicates the position of the cellular material, which appears dark due to the treatment with osmium tetroxide. The black arrow points onto the polymerised pure resin part of the block.

\subsubsection{Vitrification}

The easiest way to control the metabolic state of the bacterial cells during an $\mathrm{X}$-ray experiment is to fix it beforehand. In the field of electron microscopy numerous different fixation protocols and methods have been developed to preserve the cellular structure as best as possible 140,192. For many applications chemical fixation is sufficient. However, it is also known that chemical fixation is not 
minimal invasive and prone to damaging the native ultrastructure $70,244,323$. Currently, the near optimum preservation of the native state is considered to only be achievable by vitrification (cf. $\mid 4,5,191]$ ), i.e. rapidly cooling the fully hydrated specimen below the glass transition temperature $\mathrm{T}_{g}$ of water of about $136 \mathrm{~K}[35]^{16}$ and thereby freezing the water in an amorphous state. The prevention of crystal formation (cubic or hexagonal ice) during the process of temperature lowering is advantageous as, for instance, the formation of crystalline ice affects the concentration of solutes within the cells (phase segregation) which in turn causes structural damage [192]. In addition, the increase in volume during crystallization can be hazardous for cellular structures 244. In practise, there are two vitrification techniques that are commonly applied: (i) plunge freezing and (ii) high pressure freezing. The advantage of the plunge freezing method is that a corresponding apparatus can be easily constructed from a few simple materials (cf. [95]), whereas the high pressure freezing allows vitrification of samples with a thickness of hundreds of microns 244. In case of the plunging method, which was used for the results in this thesis, the sample itself is injected with high speed $(\sim 1-2 \mathrm{~m} / \mathrm{s})$ into a liquid cryogen such as propane or ethane, which is kept shortly above its melting point $\left(\mathrm{T}_{\text {melt }}(\right.$ ethane $)=90 \mathrm{~K}, \mathrm{~T}_{\text {melt }}($ propane $\left.)=84 \mathrm{~K}[244]\right)$.

The complete (significant) vitrification of pure liquid water and dilute aqueous solutions has been primarily demonstrated in a reproducible manner by a slightly different technique: jet-freezing 27 . The experimental procedure by Brüggeler and Mayer was to jet emulsion droplets $(1-5 \mu \mathrm{m})$ of $n$-heptane containing the sample material into liquid ethane $(\sim 90 \mathrm{~K})$. Here, the necessary cooling rate was estimated to be between $10^{5} \mathrm{~K} / \mathrm{s}$ and $10^{6} \mathrm{~K} / \mathrm{s}$. Using the plunging method, it was shown that pure water can only be vitrified when its volume is smaller than droplets with a diameter of $1 \mu \mathrm{m}$ [191. Cellular material like bacterial cells contain about $60 \%$ to $80 \%$ water but it cannot be considered to be in a pure state due to the complexity of the cytoplasma. As a consequence, the conditions for successful vitrification can often be relaxed $[244$ and also thicker specimens $(<10 \mu \mathrm{m})$ may be vitrified by plunge freezing 192 . Here it should be kept in mind that due to the complexity of vitrification of cellular material the formation of small ice crystals can never be fully excluded but their size and occurrences are definitely minimised. In the ideal case, their size is below the experimental resolution. In addition, it should be noted that the cooling rates of $10^{5} \mathrm{~K} / \mathrm{s}$ allow fixing the state of the sample within a time window that is in the millisecond range. Therefore, processes happening on shorter time scales such as chemical reactions cannot be fixed with this method.

In this thesis, plunge freezing was carried out using a commercial grid plunging machine (Leica EM GP, Leica Microsystems; cf. [309, 322 for exemplifying applications) which allows preparing the sample grid under humidity- and temperaturecontrolled conditions. In addition, the grid plunger is able to monitor and control the temperature of the cryogen. Both ethane and a mixture of $63 \%$ propane and

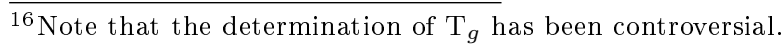


$37 \%$ ethane were used as cryogens. Their working temperatures were set to $90 \mathrm{~K}$ and $84 \mathrm{~K}$, respectively. The mixture was preferred as it yields a comparable quality of vitrification but exhibits a lower melting temperature [305]. This is particularly helpful to prevent the cryogen from freezing which simplifies the working conditions.

The plunging procedure is as follows (cf. also $[95,322]$ ):

1. Initiate the grid plunger with desired parameters (humidity, chamber temperature and cryogen temperature).

2. Prepare sample holders. It is best to hydrophilize the surface of $\mathrm{Si}_{3} \mathrm{~N}_{4}$ windows (Silson, Uk). During the blotting step this helps to keep a very thin water layer on the cells. In the ideal case, the cells are coated in a layer of about $100 \mathrm{~nm}$ of water before entering the cryogen. Hydrophillization was achieved by $5-10 \mathrm{~min}$ of plasma cleaning (Harrick Plasma, NY, USA).

3. Prepare cell suspension. There are two parameters which may be important for the experiment: (i) cell density and (ii) cell medium. For instance, a tomographic imaging experiment requires a comparably low cell density, whereas a high cell density may be more suitable for a scanning experiment. Finding the desired density involves repetitions of steps 3 and 4 , followed by an optical inspection. Typically, the cell medium is buffer $(\mathrm{pH}=7.2-7.3)$, which is fine if the X-ray experiment is carried out under cryo-conditions. However, if the sample is inspected with $\mathrm{X}$-rays in a dried state (involving lyophilisation after vitrification) buffer or growth media are not recommend as these can yield plenty of crystalline remnants (cf. Fig. 2.23). In case of endospores of B. subtilis and B. thuringiensis, good results can be obtained using deionized water.

4. Load the sample holder into the humidity chamber. Typically, the flat square side of the sample holder is aligned vertically, that is, along the plunging direction but it should be noted that horizontal alignment may yield better vitrification results 155 . A droplet of $1-1.5 \mu$ l of cell suspension is placed onto the centre of the $\mathrm{Si}_{3} \mathrm{~N}_{4}$ membrane. Although the grid plunger is equipped with an automated blotting routine it is often necessary to manually blot the excess suspension in order to achieve the thin layer of water on top of the cells. Here it should be noted that $1 \mu$ of liquid may extend up to about $40 \mu \mathrm{m}$ from a $5 \mathrm{~mm} \times 5 \mathrm{~mm}$ base (assuming a cuboid shape of the liquid on the sample holder).

5. Promptly start the plunging step.

6. Transfer the sample into a cryo vial and store under cryogenic conditions until further use. 


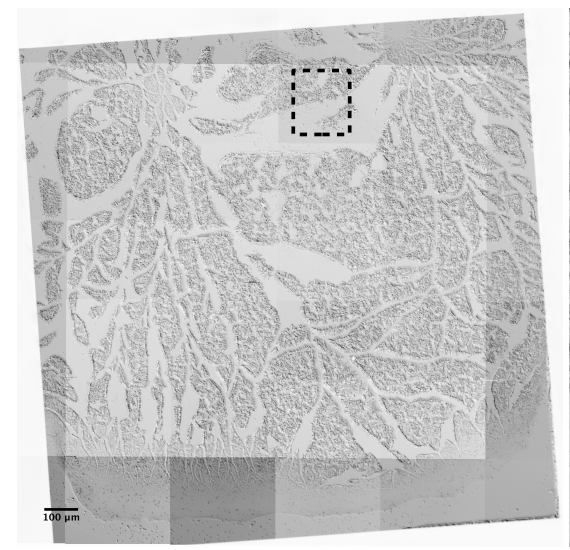

(a)

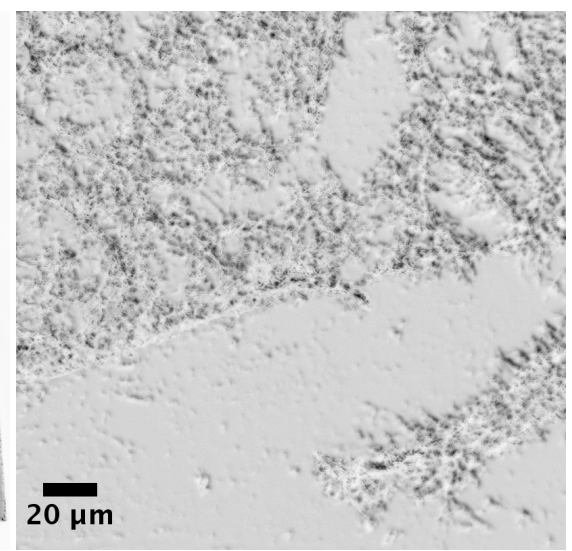

(b)

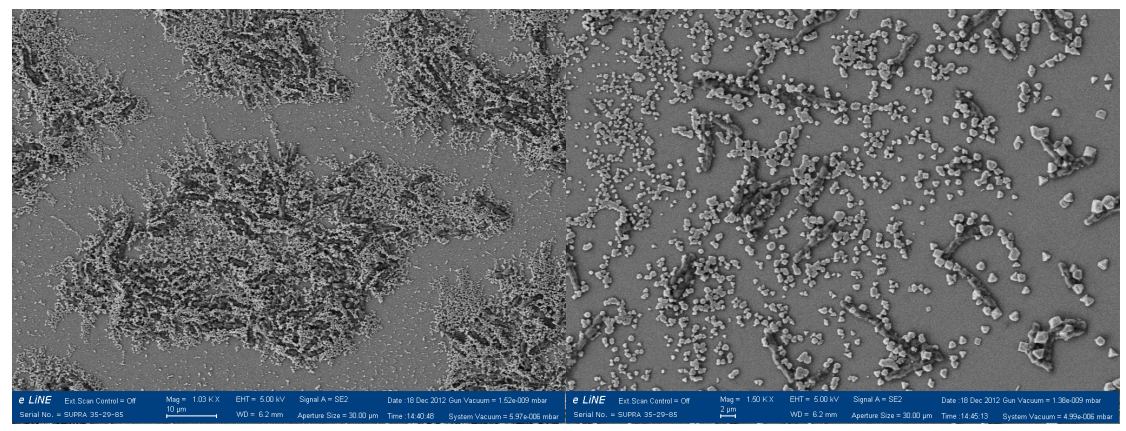

(c)

(d)

Figure 2.23: (a), (b) present images of a sample of freeze-dried endospores of $B$. subtilis that shows excessive remnants from buffer. (a) presents a stitched image of multiple optical DIC micrographs covering the whole $\mathrm{Si}_{3} \mathrm{~N}_{4}$ membrane. (b) shows a zooming-in of (a). The grey scales of the images are inverted for reasons of an optimised contrast and visibility. The issue of buffer remnants is illustrated by SEM images of air dried samples of $B$. thuringiensis on a silicon wafer in (c) and (d). Here, the rod-shaped bacteria appear comparably dark, and it can be seen that there is plenty of crystalline material surrounding the cells.

\subsubsection{Lyophilisation}

The success and the quality of lyophilisation crucially depends on maintaining the pressure below the saturation vapour pressure of (amorphous) ice while ensuring the temperature of the sample to be below the threshold for devitrification. In case of pure water, devitrification is expected around $T_{g}=136 \mathrm{~K}$, whereas according to 244 the recrystallization temperature is expected to be around $183-193 \mathrm{~K}$ for biological cells ( $233 \mathrm{~K}$ for cryoprotected cells). It is illustrative to look at the necessary sublimation times of hexagonal ice (cf. Tab. 2.6): the drying time 
of pure water being kept below the transition temperature for recrystallization appears to be unpractical. However, the saturation vapour pressure of amorphous ice should be 1-2 orders of magnitude higher $[175]$ reducing the necessary drying times to feasible values. For instance, $5 \mu \mathrm{m}$ thick vitrified biological samples may be dried in a time of about $1.5 \mathrm{~h}$ at $150 \mathrm{~K} \mid 175$. Using table 2.6 and the HertzKnudsen equation for the sublimation rate |150,175, 244, one obtains a drying time of about $0.08 \mathrm{~min}$ at $173 \mathrm{~K}$ for a sample of $1 \mu \mathrm{m}$ vitrified biological material indicating that lyophilisation without formation of cubic or hexagonal ice can be achieved for sufficiently thin samples such as bacteria. In particular, the HertzKnudsen equation is given by 150

$$
J_{s}\left[\mathrm{~g} / \mathrm{cm}^{2} \mathrm{~s}\right]=p_{t r} \sqrt{M /\left(2 \pi R T_{t r}\right)}
$$

where $M=18.0152 \mathrm{~g} / \mathrm{mol}$ is the molecular weight of evaporating water, $R=$ $8.314471 \cdot 10^{7} \mathrm{~J} /(\mathrm{K} \cdot \mathrm{mol})$ is the universal gas constant, $T_{t r}$ is the absolute temperature in kelvins, $\mathrm{K}$, at the interface and $p_{t r}$ is the saturation vapour pressure, in $10^{-1} \mathrm{~Pa}$, at $T_{t r}$.

Lyophilisation of the bacterial samples was carried out in a workshop-build freeze drying apparatus which is designed for reaching high vacuum (for details see [239). The forepump system consists of a rotary vane pump. At lower pressures (< $10^{-5}$ bar), the high vacuum is maintained by a cryopump. The low temperature reservoir is a massive $26 \mathrm{~kg}$ brass block that is initially cooled with liquid nitrogen to about $77.15 \mathrm{~K}\left(196^{\circ} \mathrm{C}\right)$. The brass block is formed with a small bassin that is loaded with the samples bathing in liquid nitrogen. To this end, it is possible to record automatically the raise in temperature of the block (cf. Fig. 2.24) but the pressure can only be monitored in an analogue fashion. Monitoring the pressure indicates that a high vacuum of $\sim 10^{-8}$ bar can be established after about 4 hours. Within this time the brass block heats up to approx. $143 \mathrm{~K}$ (cf. Fig. 2.24). Thereafter, it takes another 6 hours before reaching the critical temperature of $90 \mathrm{~K}$. Hence, it is reasonable to assume that there is sufficient time for freezedrying without devitrification of vitrified bacterial samples with a thickness of $\lesssim 5 \mu \mathrm{m}$.

Examples of samples that have been plunge-frozen and lyophilised are presented in figures $2.25,2.26$ and 2.27 .

Table 2.6: Drying times for hexagonal ice. $P_{s}$ is the saturation vapour pressure. Data taken from 244 .

\begin{tabular}{clc}
\hline $\mathrm{T}[\mathrm{K}]$ & $P_{s}[\mathrm{bar}]$ & Drying time $/ \mu m$ \\
\hline 123 & $6.7 \cdot 10^{-15}$ & 27 years \\
153 & $1.2 \cdot 10^{-10}$ & 14 hours \\
173 & $1.3 \cdot 10^{-8}$ & $8 \mathrm{~min}$ \\
183 & $9.3 \cdot 10^{-8}$ & $1 \mathrm{~min}$ \\
\hline
\end{tabular}




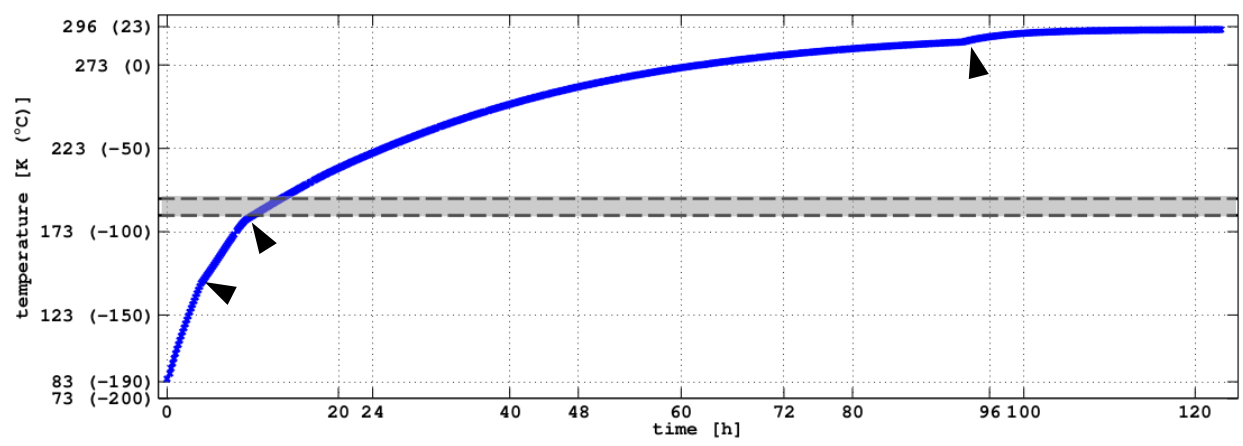

Figure 2.24: The graph shows an exemplifying curve of the rise in temperature during lyophilisation. The black arrow heads mark the points $\left(t[\mathrm{~h}], T\left[\mathrm{C}^{\circ}\right]\right)=$ $(3.98,-130.9),(9.15,-93.1),(92.98,13.9)$ which supposedly indicate switching of the pumping system to high vacuum $\left(\sim 10^{-8}\right.$ bar $)$, end of sublimation and opening of the vacuum chamber to room temperature, respectively. The grey zone indicates the temperature region between $183 \mathrm{~K}$ and $193 \mathrm{~K}$ where devitrification is expected. 


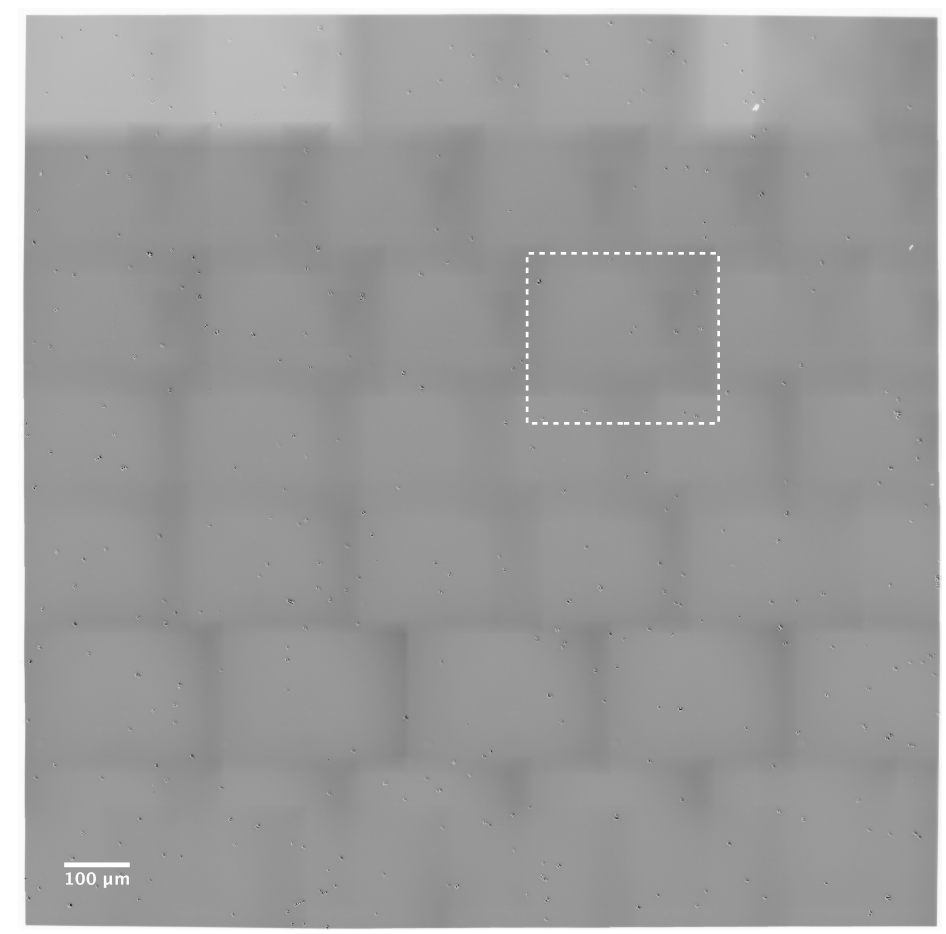

(a)

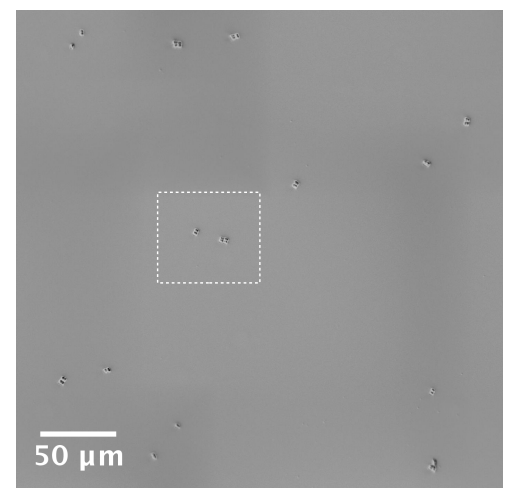

(b)

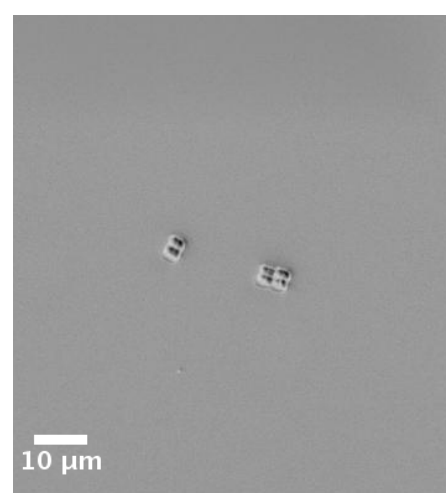

(c)

Figure 2.25: This figure shows images of the sample of freeze-dried $D$. radiodurans that have been analysed with a combination of ptychography and tomography (cf. section 3.1. $\mathrm{p}$.69). (a) presents a stitched image of multiple optical DIC micrographs covering the whole $\mathrm{Si}_{3} \mathrm{~N}_{4}$ membrane. (b) shows a zooming-in of (a) and (c) is a zooming-in of (b) as indicated by white, dashed frames. The grey scales of the images are inverted for reasons of an optimised contrast and visibility. The image in (a) was created by using the MosaicJ plugin of the open-source ImageJ software project which was originally programmed by Wayne Rasband $\mid$\begin{tabular}{l|l|l|l||}
237 & 261 & 262 & 292
\end{tabular} . 


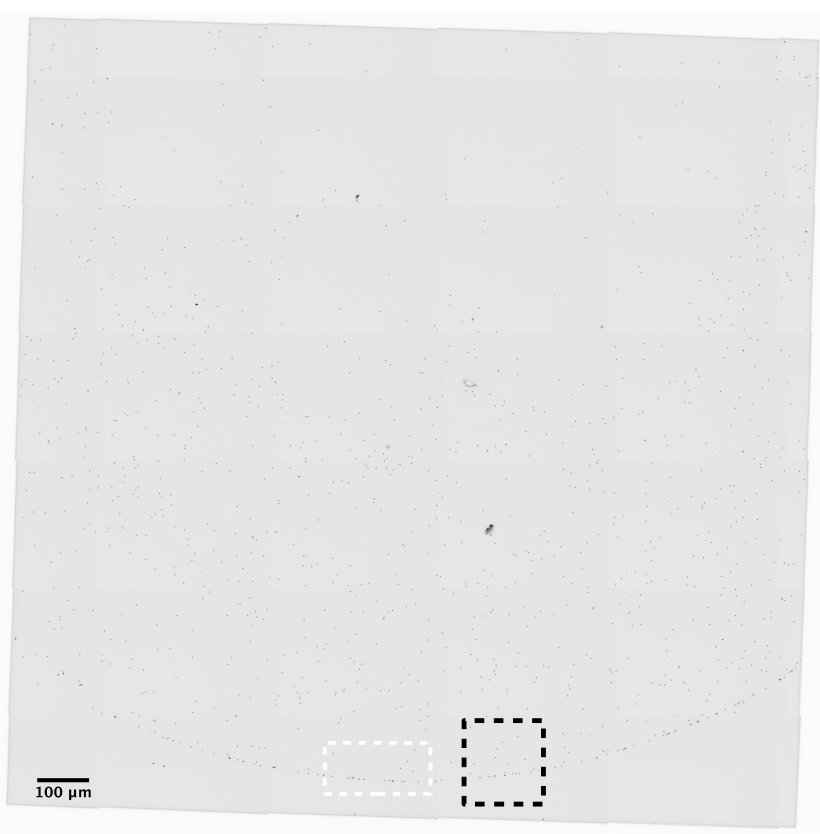

(a)

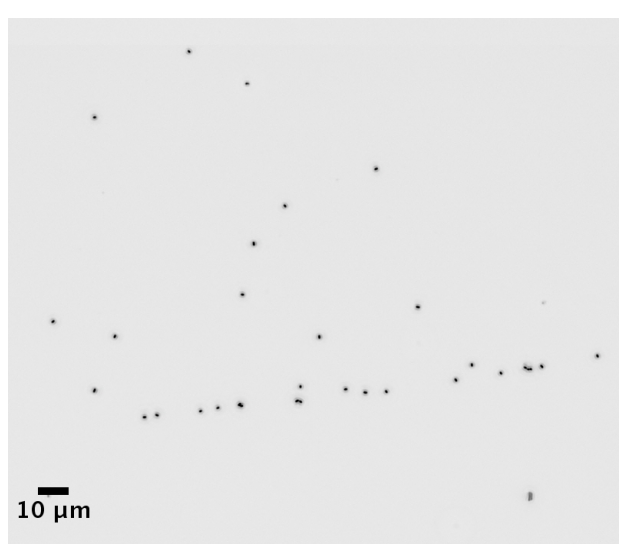

(b)

Figure 2.26: This figure shows images of the sample of freeze-dried endospores of B. subtilis on a $\mathrm{Si}_{3} \mathrm{~N}_{4}$ membrane (cf. section 3.5 p. 161). The dashed, white frame indicates the region where X-ray experiments were carried out. (a) presents a stitched image of multiple optical DIC micrographs covering the whole membrane. (b) shows a zooming-in of (a) as indicated by the black, dashed frame. The black spots are single endospores. The grey scales of the images are inverted for reasons of an optimised contrast and visibility. The image in (a) was created by using an automated scanning stage for the inverse Microscope Axio Observer Z1 in combination with the software AxioVision (Zeiss, Deutschland). 


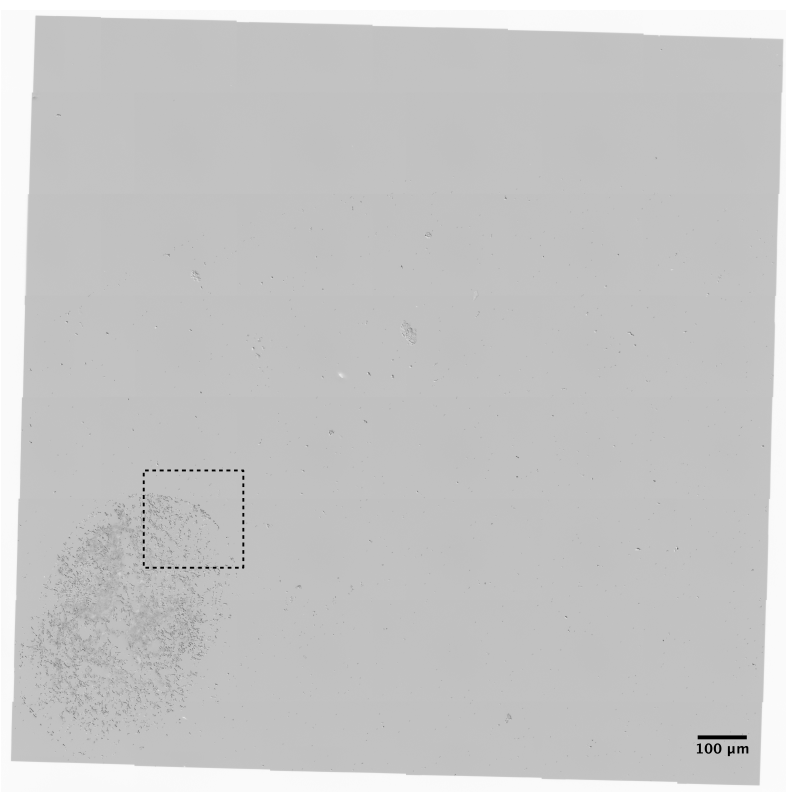

(a)

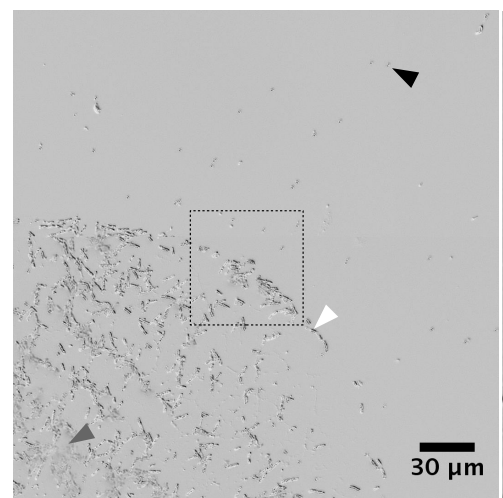

(b)

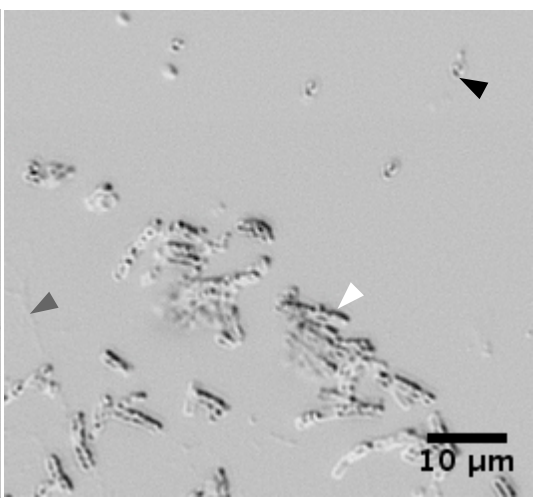

(c)

Figure 2.27: This figure shows images of the sample of freeze-dried B. thuringiensis cells and endospores on a $\mathrm{Si}_{3} \mathrm{~N}_{4}$ membrane (cf. section 3.5 p. 161). (a) presents a stitched image of multiple optical DIC micrographs covering the whole membrane. (b) and (c) show a zooming-in of (a) and (b) as indicated by the black, dashed frames, respectively. The black arrow heads indicate isolated endospores, whereas white arrow heads point onto cell material. The grey arrow heads identify regions with remainders from the medium and buffer. The grey scales of the images are inverted for reasons of an optimised contrast and visibility. The image in (a) was created by using an automated scanning stage for the inverse Microscope Axio Observer Z1 in combination with the software AxioVision (Zeiss, Deutschland). 


\section{Experiments}

\subsection{Ptychographic Imaging and Nano-Diffraction of $D$. $r a-$ diodurans}

Ptychographic coherent X-ray diffractive imaging (PCDI) has been combined with nano-focus X-ray diffraction to study the structure and density distribution of unstained and unsliced bacterial cells $D$. radiodurans using a Fresnel zone plate (FZP) nano-focus. In comparison to PCDI, which necessitates a high degree of coherence, overlap and oversampling, nano-diffraction can be carried out with relaxed experimental constraints. While PCDI provides images of the bacteria with quantitative contrast in real space with a resolution well below the beam size at the sample, spatially resolved small angle X-ray scattering using the same FZP (cellular nano-diffraction) provides structural information at highest resolution in reciprocal space up to $2 \mathrm{~nm}^{-1}$.

In principle, cellular nano-diffraction can be used to access many structural parameters such as inter-molecular spacings, short range correlations, lattice constants and form factors. In contrast to the seminal works by Fratzl and coworkers dealing with small angle X-ray scattering (SAXS) of biological materials $87,88,106,157,171,224,248,318$, where the average is taken over the area of a much larger beam, the nano-focus restricts the average over Fourier components to a much smaller volume. This may be particularly useful for studying biological cells, which exhibit a local ordering on small length-scales ${ }^{17}$. For obvious reasons, one aims at reducing unwanted scattering contributions from the surrounding cellular material. Importantly, finding these cellular structures or interpreting the results may be significantly simplified by a combination with a high resolution imaging technique such as ptychography. Here, it is shown how the real and reciprocal space approach can be used synergistically on the same sample and with the same set-up.

This section is partly based on a work published in $\mid 330$. Note that, the presentation here reflects the original intention for publication. The ptychographic imaging results of the bacterial cells, which are presented in section 3.1 .9 (p. 97), have not been considered to be significant enough to warrant publication in [330|. For this reason, the ptychographic imaging experiment was repeated and improved on a different cellular sample (section 3.1.10, p. 100). Additionally, some more technical details about the tomographic alignment are included. Furthermore, transmission electron microscopy was carried out on cells of $D$. radiodurans. An investigation of the influence of the spatial coherence on the ptychographic reconstructions is also included in section 3.1.8. The presentation here is intended to supply a more complete and coherent picture of the experiment. In case of the cellular nano-diffraction experiment, M. Priebe contributed significantly to the experimental part and he also prepared the corresponding bacterial sample which is gratefully acknowledged.

${ }^{17}$ For introductions to (standard) SAXS, the reader is referred to 99107 . 


\subsubsection{Experimental Set-up}

The experiment has been carried out at the coherent Small Angle X-ray Scattering beamline (cSAXS) of the Swiss Light Source (SLS) at the Paul Scherrer Institut in Villigen, Switzerland. Starting from the undulator source consisting of $N_{u}=96$ magnet periods with undulator period of $19 \mathrm{~mm}$, the beam was defined by the following optical elements, as indicated schematically in Fig. 3.28: (i) horizontal slits S0 at $12.1 \mathrm{~m}$ distance from source, (ii) horizontal and vertical slits $S 1$ at $26 \mathrm{~m}$, (iii) a $\mathrm{Si}(111)$ double crystal monochromator at $28.6 \mathrm{~m}$ (2nd crystal). The first crystal was $\mathrm{LN}_{2}$-cooled. The monochromator was set to a photon energy of $6.2 \mathrm{keV}$. Following (iv) further slit systems S2, S3, and S4 as well as the fast shutter system (FS) for triggered detector read-out, the beam impinges on a Fresnel zone plate (FZP) with preceding central stop (CS). A highly efficient FZP fabricated of Au on a $\mathrm{Si}_{3} \mathrm{~N}_{4}$ membrane using $100 \mathrm{keV}$ e-beam lithography and electroplating 102,103 with a diameter of $\varnothing=200 \mu \mathrm{m}$, zone height of $1 \mu \mathrm{m}$ and outermost zone width $\mathrm{dr}=100 \mathrm{~nm}$ was used here. The FZP was located at about $34 \mathrm{~m}$ from the source and at a distance of about $0.1 \mathrm{~m}$ to the sample. An order sorting aperture (OSA) with a diameter of $\varnothing=20 \mu \mathrm{m}$ was placed between FZP and sample. The sample was positioned by a high precision piezo stage (Physik Instrumente, Germany) mounted on an air bearing rotation stage (Micos UPR160F) on top of a hexapod (PI M-850).

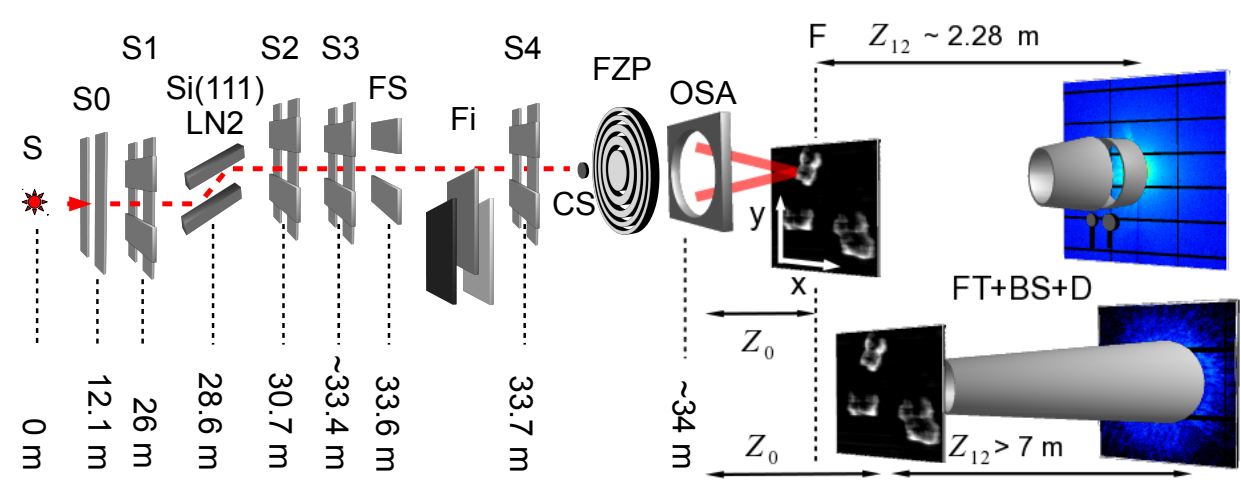

Figure 3.28: Beamline setup used for cellular diffraction and ptychography: X-ray undulator source $(\mathrm{S})$, horizontal slits (S0), horizontal and vertical slits (S1-S4), Si(111) monochromator ( $\mathrm{LN}_{2}$-cooled), fast shutter (FS), filter (Fi), central stop (CS), Fresnel zone plate (FZP), order sorting aperture (OSA) and focus (F) with bacterial specimen. Set-up for cellular nano-diffraction: sample in focus, short distance $z_{12}=2.28 \mathrm{~m}$ to detection plane (D), short flight tube (FT) and beam stops (BS). PCDI setup: sample slightly out of focus $z_{z 01}=1 \mathrm{~mm}$, long distance $z_{12}=7.22 \mathrm{~m}$ to detection plane (D) and long flight tube (FT).

The first part of the experiment was optimised for diffraction: a cryojet was used to cool the sample and the zero read-out noise photon counting detector PILATUS 2M (SLS detector group, [163] ) was placed $z_{12} \simeq 2.28 \mathrm{~m}$ behind the sample 
(according to a calibration using silver behenate), with $1475 \times 1679$ pixels of size $172 \mu \mathrm{m} \times 172 \mu \mathrm{m}$. A flight tube of length $2.063 \mathrm{~m}$ was used between sample and detector to reduce air-scattering of the diffracted X-ray beam. Beam damage of the detector was suppressed by using multiple beam stops between sample and detection plane and/ or by using silicon filters of appropriate thickness. The coherence properties of the beam were altered by changing the gap of the horizontal slits S0. Importantly, increasing the gap yields a beam with lower spatial coherence, but more flux.

The second part of the experiment was optimised for ptychography: The coherent slit setting of S0 (gap width $10 \mu \mathrm{m}$ ) was chosen. In addition, a long distance between sample and detection plane is favoured for sufficient sampling of the recorded diffraction pattern (fulfilment of the oversampling condition). Therefore, the PILATUS $2 \mathrm{M}$ was placed at the long working distance of $z_{12} \simeq 7.22 \mathrm{~m}$ behind the sample and a helium-filled flight tube of length $7 \mathrm{~m}$ was used. Ptychographic imaging results of $D$. radiodurans as presented in section 3.1 .10 (p. 100) have been obtained during a second experiment at the cSAXS beamline using the same experimental setup. However, the detector was placed at $z_{12} \simeq 7.65 \mathrm{~m}$ (see 217 for distance calibration) with an air path of $\simeq 0.4 \mathrm{~m}$ between the flight tube and the PILATUS. A cryojet, beam stops and filters were not used for ptychographic imaging.

\subsubsection{Sample Preparation}

Cells of Deinococcus radiodurans R1 were cultivated from freeze-dried cultures (DSM No. 20539 by the German Collection of Microorganisms and Cell Cultures) for one day at $37^{\circ} \mathrm{C}$ on petri dishes covered with nutrient medium Corynebacterium Agar (Table B.1, p. 192). Prior to preparation, the actively growing cells were washed off the culturing medium with about $1.5 \mathrm{ml}$ buffer solution $(2 \mathrm{~g} / \mathrm{l}$ $\mathrm{KH}_{2} \mathrm{PO}_{4}, 0.36 \mathrm{~g} / 1 \mathrm{Na}_{2} \mathrm{HPO}_{4} \cdot 2 \mathrm{H}_{2} \mathrm{O}, \mathrm{pH}=7.2$ ). After placing a droplet of cell suspension onto the substrate, a polyimide foil of $12.5 \mu \mathrm{m}$ thickness (MiTeGen, USA), the cells were allowed to adhere for 60 seconds. In case of the second ptychographic imaging experiment of section 3.1 .10 (p. 100), the cells were placed on a $\mathrm{Si}_{3} \mathrm{~N}_{4}$ membrane of $500 \mathrm{~nm}$ thickness (Silson, UK) and allowed to adhere for a few seconds. The remaining buffer was blotted, both types of foils were cryoplunged into liquid ethane to prevent crystallization and afterwards lyophilized in a home-built freeze-drier.

\subsubsection{Details and Terminology: Cellular Nano-Diffraction}

In a first step diffraction data of $D$. radiodurans cells was taken by translating the object in the XY-plane of the focal region using a coherent slit setting of S0 on the sample region A of Fig. 3.29 (see Table 3.7 for a summary on scan details). The performed mesh scans differ in dwell time $\Delta T$, step size $\Delta_{x y}$ between adjacent scan points and field of view (FOV). The dataset $A_{1}$ was recorded without a beam stop but with a Si filter of $50 \mu \mathrm{m}$ thickness. $A_{2}, A_{3}, A_{4}$ were carried out with a 
beam stop and no filters. The two diffraction patterns of $A_{4}$ were taken on a cell region and on an empty region of the polyimide foil, respectively.

Secondly, more diffraction data was taken with the same settings on the sample region B (cf. Fig. 3.29, Table 3.7). However, a rather incoherent beam was used by opening the slits S0 to a width of $\Delta=0.42 \mathrm{~mm}$. Two beam stops were used during recording of the datasets $B_{1}$ and $B_{3}$. Afterwards, the scan $B_{2}$ was performed in the coherent setting without any beam stop but with a Si filter of $50 \mu \mathrm{m}$ thickness.

For each mesh scan four contrast values can be calculated from every single diffraction pattern: the integral signal (transmission), the ratio of the integral signals from a region defining the central beam and from its counter set (darkfield) and the differential phase contrast in the $\mathrm{x}$ - and $\mathrm{y}$ - direction, DPC-x and DPC-y respectively (cf. section 1.3 .1 , p. 34). Note that by blocking a part of the primary beam using a beam stop, none of these contrasts reflect quantitative measurements any more. However, the tails of the primary beam can still be used for the analysis to obtain sufficient contrast. Here, only a subset of $194 \times 194$ pixels around the centre of the beam of each diffraction pattern has been used to obtain the different contrasts.

Next, the corresponding diffraction patterns were segmented according to the contrast (darkfield or DPC) into 'cell and sample holder', $\Sigma_{1}$, and 'sample holder', $\Sigma_{2}$, (cf. e.g. subgraphs in Fig. 3.30, 3.31). This was done either by thresholding the darkfield image or by thresholding a contrast image followed by further image processing. Diffraction curves $I(|\vec{q}|)$ have been calculated for both sets of diffraction patterns by averaging over the set $\langle\cdot\rangle_{\Sigma_{i=1,2}}$ and azimuthally averaging $\langle\cdot\rangle_{\phi}$ with respect to constant radii. The difference signal

$$
\Delta I(|\vec{q}|)=\left\langle\left\langle I_{j}(\vec{q})\right\rangle_{\phi}\right\rangle_{j \in \Sigma_{1}}-\left\langle\left\langle I_{j}(\vec{q})\right\rangle_{\phi}\right\rangle_{j \in \Sigma_{2}}
$$

is the basis for the analysis. Here, the momentum transfer $|\vec{q}|=2 k \sin \alpha / 2$ is used with $\alpha$ being the angle between optical axis and scattering vector, and $\mathrm{k}$ denoting the wavenumber. Due to spurious OSA scattering, some sectors of the diffraction patterns had to be excluded. Dead and hot pixels have been masked. The absence of correlations between the scattering signal from the sample holder and the scattering of the cellular specimen justifies background subtraction similar to conventional (incoherent) diffraction. The fact that both coherent and incoherent illumination settings yield consistent results can be regarded as a validation of this assessment. It is stressed that background subtraction based on a pixel by pixel analysis of the sample (sample pixels versus background pixels) was found to be significantly more robust than background subtraction based on a second empty sample.

\subsubsection{Results: Cellular Nano-Diffraction}

The darkfield and differential phase contrast of the scanned regions $\mathrm{A}$ and $\mathrm{B}$ clearly reveal the outline and some internal structure of the cells in the scanned FOV (see Fig. 3.29). The images of the sample regions A and B show typical shapes of $D$. radiodurans aggregates, as known from light and electron microscopy 
$[77,169,342$ and a previous ptychographic study [97]). Importantly, scanning diffraction with the four observables transmission, phase shift DPC-x, DPC-y and darkfield allows for fast, robust visualisation of biological cells without staining and with the additional benefit of a large FOV that can be easily adapted. The images have been recorded with samples kept in the cryojet, demonstrating that cryo samples can be made compatible with the vibration requirement needed for nano-beam scanning diffraction. In case of the coherent datasets ptychography yields an accurate picture of the actual illuminating wave-field. The reconstructed wave-field $P(\vec{x})$ (Fig. 3.33(a)) was propagated along the optical axis back to the position of this sample. Here, the advantage of combining scanning diffraction images with ptychographic phase retrieval is stressed, since the probe and hence the resolution can be exactly quantified, presenting a significant advantage over conventional knife edge measurements, which will be discussed in section 3.1.7 (p. 83). The probe at the sample is illustrated by the overlay in Fig. 3.29(d). The size of the beam at the sample matches the visual impression of the resolution.

One may be concerned about the effect which a highly convergent beam has on small-angle X-ray scattering, since SAXS measurements usually require collimated beams. By placing a sample in the planar wave-front of the focal plane, the farfield pattern of the sample structure is measured. More precisely, the measured far-field pattern corresponds to the Fourier transform of the sample convolved with the Fourier transform of the probe, which is a plane wave with for example a Gaussian envelope in the focal plane. Since for cellular nano-diffraction one is by definition interested in diffraction from structures, which are smaller than the beam size, one realizes that the convolution of the far-field pattern with the Fourier transform of the probe has only a negligible effect, if the structure size is much smaller than the beam size. This is the case in the present work. Furthermore, one may even argue that it will be easier to control the wave-front flatness of a coherent nano-beam by ptychography than the collimated (stochastic) wave-fronts of macroscopic SAXS measurements. For instance, the reconstructed probe can be used to precisely find the position of the focus in the beam.

Next the diffraction data in q-space is discussed, which can give complementary information to the ptychographic reconstruction, in particular since a larger qrange can be exploited. At the same time, the ptychographic reconstruction helps to 'locate' the source of the diffraction signal. Since each point of the images (differential phase contrast and darkfield) corresponds to a single diffraction pattern the image can be segmented into pixels with cells and without cells, representing the empty sample holder (see insets in Fig. 3.30, 3.31). By making use of the whole sets of collected diffraction data one is able to discriminate diffraction signal of cellular material from diffraction signal of the polyimide foil, representing the background signal. The two diffraction intensities (averaged over the number of corresponding pixels) are displayed as a function of $q$ after azimuthal averaging, together with the difference signal, which can be interpreted as the average background subtracted diffraction signal of the bacterial cells. These diffraction curves can be well described by a power-law behaviour $y=a \cdot q^{\nu}+c$ in the high q-region (Fig. 3.30, 3.31). All exponents $\nu$ lie between values of -3 and -4 . Importantly, 
this is also true for the diffraction curves that have been obtained at singular positions $\left(A_{4}, B_{3}\right.$, table 3.7). One might expect the diffraction signal to be quite different at a single position of the cell in comparison to the case where the diffraction signal is averaged over a whole cell or a bunch of cells. However, for the case of a bacterial cell, without the pronounced organelles typical for eukaryotic cells, the results show that this is not really the case. In other words, the structure leading to the observed power law decay originates at length scales much smaller than the beam size used here $(\sim 508 \mathrm{~nm} \times 538 \mathrm{~nm}$ FWHM values of probe intensity $\mathrm{h} \times \mathrm{v})$. Thus a single shot represents a spatial average very similar to that of the entire cells. The fact that the functional form of the diffraction signal follows a power law with an exponent $-4 \leq \nu \leq-3$ has been observed for the power spectral density of a ptychographic reconstruction of $D$. radiodurans $\mid 97$ and is indicative of a self-affine distribution of scattering length density. Note that structures composed of compact regions with sharp interfaces would exhibit a power law with $\nu=-4$, corresponding to the well known Porod law. Least-square fits are used as shown in Fig. 3.30 to determine the cut-off $q_{\dagger}$ where the diffraction signal reaches a constant white noise level. This cut-off can be defined as the resolution of the diffraction experiment. In the following it can be used to compare the influence of (a) counting time and (b) slit settings on $q_{\dagger}$. In explicit terms $q_{\dagger}$ is the intersection of the power-law decay $a \cdot q^{\nu}$ and the constant background $c$

$$
q_{\dagger}:=(c / a)^{1 / \nu}
$$

The upper graph in Fig. 3.30 shows the background corrected cellular diffraction signal as measured for $\Delta T=1 \mathrm{~s}$ and $\Delta T=10 \mathrm{~s}$, leading to $q_{\dagger}=0.75 \mathrm{~nm}^{-1}$ and $q_{\dagger}=0.95 \mathrm{~nm}^{-1}$, respectively. This is in agreement to experimental expectation. The $\Delta T=100 \mathrm{~s}$ dataset recorded at a single spot, displayed in the lower graph of Fig. 3.30 shows an even stronger signal, extending to $q_{\dagger}=1.88 \mathrm{~nm}^{-1}$. This demonstrates that cellular structure can be analysed by diffraction at resolutions (in rec. space) which are currently not accessible by X-ray microscopy, neither in the conventional form nor the more recent Coherent Diffractive Imaging (CDI) variants. The data shown in Fig. 3.30 also illustrates the difficulties associated with spurious scattering by the OSA, which is difficult to suppress due to thermal effects induced by the cryojet.

Finally, it is noted that at small $q$ the $A_{4}$ dataset shows a transition of the power law decay to a plateau (not fully reached due to the BS). This behaviour was captured by adapting the fitting function to $I=a \cdot q^{\nu}\left(1-\exp \left(-(d \cdot q / 2 \pi)^{2}\right)\right)+c$, with $d$ denoting the crossover. The resulting value of $d \approx 60 \mathrm{~nm}$ gives a typical length scale of the largest structure.

Next, the effect of opening the slit $S 0$ and the associated relaxation of coherence on the resolution is discussed. Note that the high flux does not necessarily have to result in higher $q_{\dagger}$ due to a possible decrease in signal to noise. However, the results shown in Fig. 3.31 of the $B_{1}$ dataset show $q_{\dagger}=2.3 \mathrm{~nm}^{-1}$ which is indeed the highest value obtained. Furthermore, it seems that dose fractionation over the entire aggregate of cells presents an advantage over a single point long 
measurement where a moderate increase in resolution (in $q$-space) can be observed, at least for detectors which are free of read-out noise.

The results of the dose estimations are presented in Fig. 3.38, as described in the method section (cf. Eq. $(3.120)$ ). Note that the dose estimations are spatially more precise than a simpler estimation based on an average fluence, which can be best seen in Fig. 3.3\&|(b). After all, a comparison of the attainable resolution in diffraction settings should always be accompanied by a corresponding comparison in dose in view of the important issue of radiation damage in biological specimens. In case of the sample region A (coherent setting) the average photon flux was measured with the PILATUS to be $\sim 7 \cdot 10^{8}$ photons $/ \mathrm{s}$. Assuming the same photon flux at each scan position, doses of $3.6 \cdot 10^{7} \mathrm{~Gy}, 2.2 \cdot 10^{7} \mathrm{~Gy}$ and $1.3 \cdot 10^{8}$ Gy for $A_{2}$, $A_{3}$ and $A_{4}$ are obtained, respectively. In case of the incoherent setting of sample region $B$, the increase in flux is determined to be in the range of a factor of $10-17$ due to the increased gap of the slit $S 0$. Therefore, assuming an average photon flux of $\sim 10^{10}$ photons/s yields applied doses of $5.5 \cdot 10^{7}$ Gy and $2 \cdot 10^{9}$ Gy in case of the incoherent datasets $B_{1}$ and $B_{3}$, respectively. The results are summarized in Table 3.7.

Table 3.7: Overview of diffraction datasets according to dwell time $\Delta T$, step size $\Delta_{x y}$ and number of scan points $N_{x} \times N_{y}$ within the rectangular mesh. In addition, Si filter thickness (BS denotes usage of beam stops instead of Si filter) and nominal gap of slits S0 are shown as well as the results from diffraction analysis for the exponent $\nu$ of the diffraction curves, highest scattering vector $q_{\dagger}$ and dose estimations $D$.

\begin{tabular}{lcccccccc} 
region $\mathrm{A}$ & $\Delta T[\mathrm{~s}]$ & $\Delta_{x y}[\mu \mathrm{m}]$ & $N_{x} \times N_{y}$ & $\mathrm{Si}[\mu \mathrm{m}]$ & $\mathrm{S} 0[\mu \mathrm{m}]$ & $\nu$ & $D[\mathrm{~Gy}]$ & $q_{\dagger}\left[\frac{1}{\mathrm{~nm}}\right]$ \\
\hline$A_{1}$ & 0.1 & 0.1 & $161 \times 191$ & 50 & 10 & - & - & - \\
$A_{2}$ & 1 & 0.2 & $41 \times 41$ & $\mathrm{BS}$ & 10 & -3.2 & $3.6 \cdot 10^{7}$ & 0.75 \\
$A_{3}$ & 10 & 0.8 & $11 \times 11$ & $\mathrm{BS}$ & 10 & -3.7 & $2.2 \cdot 10^{7}$ & 0.95 \\
$A_{4}$ & 100 & 200 & $2 \times 1$ & $\mathrm{BS}$ & 10 & -3.6 & $1.3 \cdot 10^{8}$ & 1.88 \\
region B & & & & & & & & \\
\hline$B_{1}$ & 0.1 & 0.2 & $61 \times 61$ & $\mathrm{BS}$ & 420 & -3.5 & $5.5 \cdot 10^{7}$ & 2.30 \\
$B_{2}$ & 0.1 & 0.2 & $61 \times 61$ & 50 & 10 & - & - & - \\
$B_{3}$ & 100 & 200 & $2 \times 1$ & $\mathrm{BS}$ & 420 & -3.8 & $2 \cdot 10^{9}$ & 1.89 \\
\hline
\end{tabular}




\section{Region A}
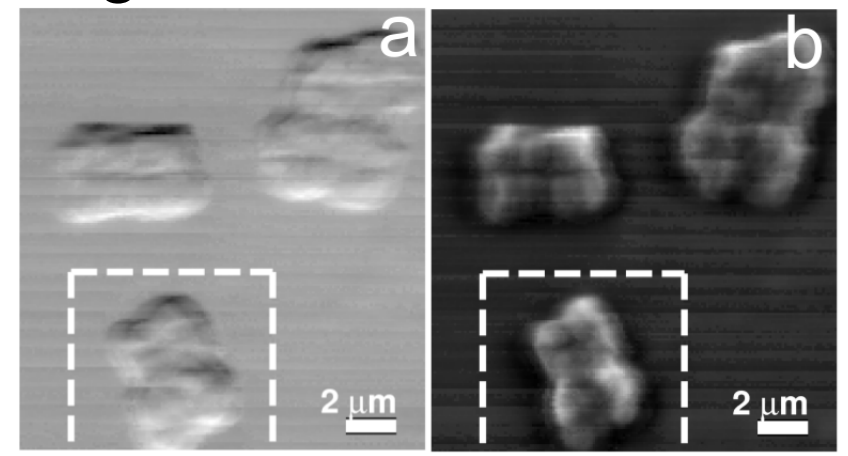

\section{Region B}
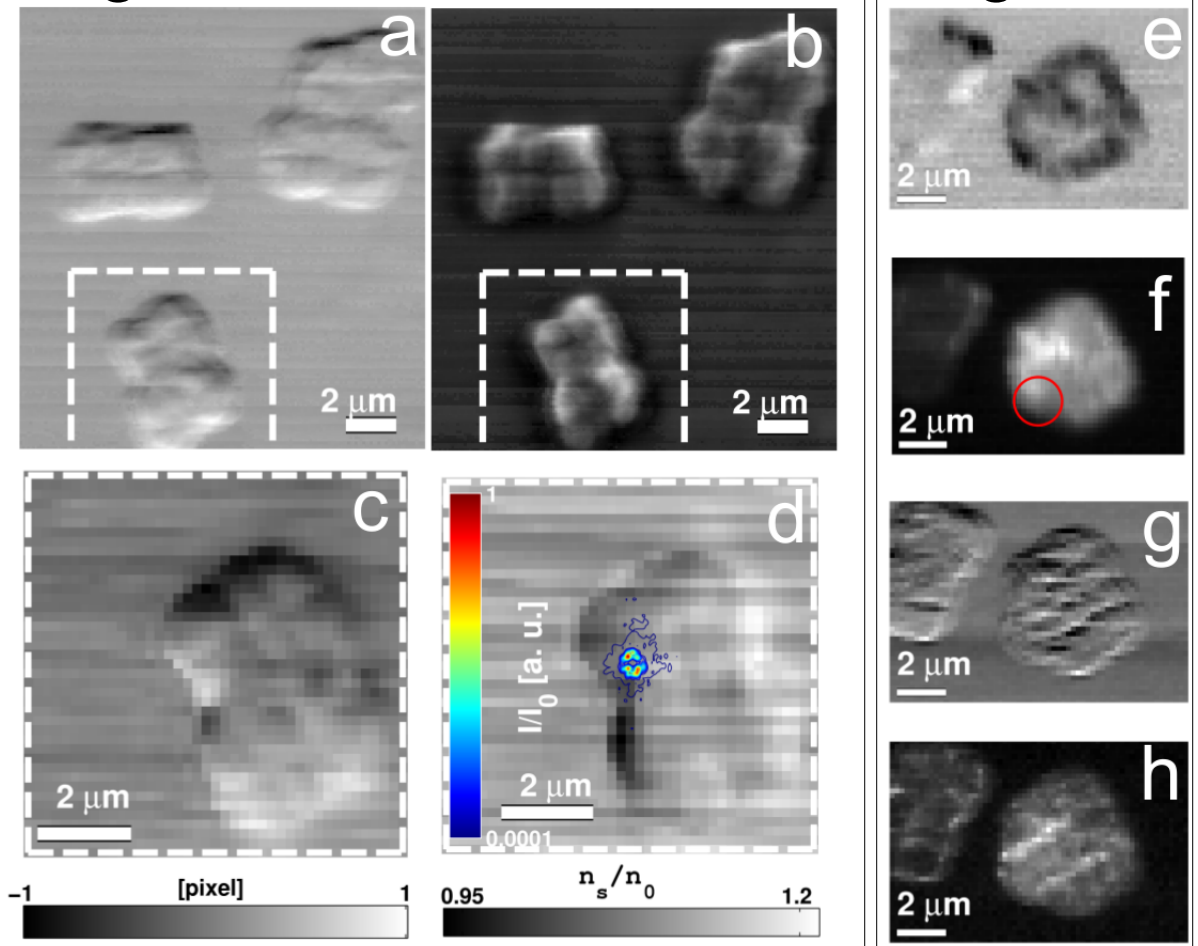

Figure 3.29: Sample region A - coherent setting: differential phase contrast (a), (c) and darkfield (b), (d) of cells are shown for the mesh scans $A_{1}$ (top) and $A_{2}$ (bottom) - (cf. Table 3.7). The colourbar in (c) denotes the movement of the first moment whereas in (d) the grey scale denotes the ratio of scattered to unscattered photons, $n_{s}$ and $n_{0}$ respectively. The intensity of the beam from the ptychographic reconstruction of the Siemens star (cf. Fig. 3.33) after back propagation of $1 \mathrm{~mm}$ into its focus is visualized on top of the darkfield (d). Note, that the propagation of $1 \mathrm{~mm}$ corresponds to the lateral displacement of the sample between the two different measurements $( \pm 100 \mu \mathrm{m}$ due to an uncertainty of the position on the sample holder with respect to the curvature of the polyimide foil). The centre of the reconstructed beam is chosen to be at the cell-position of $A_{4}$ (cf. Fig. 3.30). Sample region B - incoherent setting: a section of the scan $B_{1}$ is shown as darkfield (f) and differential phase contrast (e). The position of the cell diffraction of $B_{3}$ is indicated on top of the incoherent darkfield as red circle (f). Sample region $\mathbf{B}$ - coherent setting: darkfield $(\mathrm{h})$ and phase contrast $(\mathrm{g})$ of a corresponding coherent mesh scan $B_{2}$ are shown. Numerical simulations C: synthetic cell sample (top) and calculated differential phase contrast images (below) according to Eq. (3.109) using the back propagated probe (cf. Fig. 3.33) at different positions on the optical axis. The distance of propagation is displayed and the corresponding intensity of the probe is visualised as an overlay using the same colourbar as in (d). 

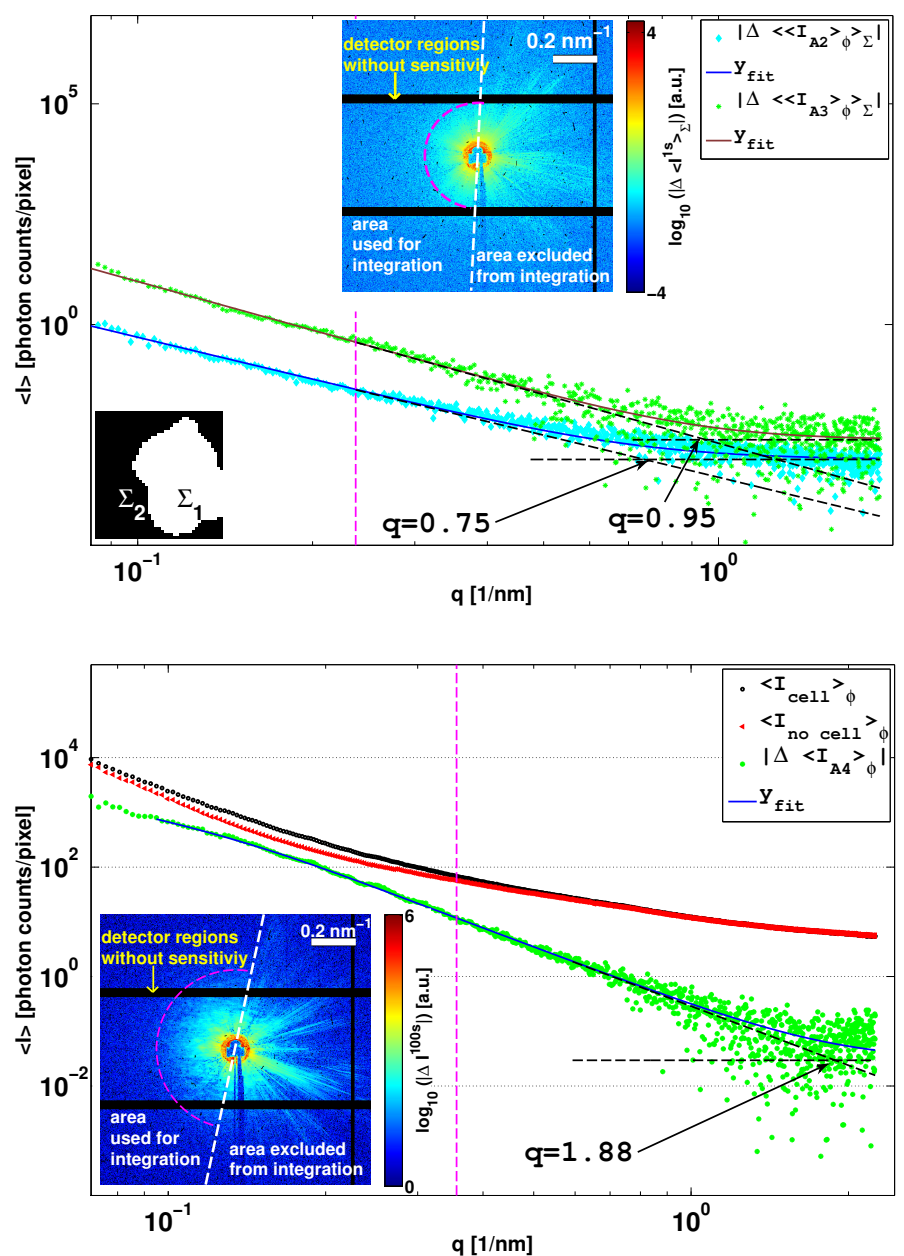

Figure 3.30: Sample region A - coherent setting: (top) Diffraction data of $A_{2}$ and $A_{3}$ (cf. Table 3.7). The sets of diffraction patterns were segmented into areas with cell signal, $\Sigma_{1}$, and without cell signal, $\Sigma_{2}$, using the differential phase contrast image of $A_{2}$ (see inset). The difference between the two classes of diffraction sets averaged according to their set size is depicted next to the legend in the top right corner for $A_{2}$. Diffraction signals were obtained by azimuthally averaging each diffraction pattern of a scan and thereafter averaging according to their segmentation. The segmented diffraction curves of $A_{2}$ are shown and the difference signals are presented for both scans in combination with their power-law fits. Exponents of $\nu=-3.2$ and $\nu=-3.7$ were obtained for $A_{2}$ and $A_{3}$, respectively. (Bottom) presents the results of $A_{4}$ (cf. Fig. 3.29 (d)). The diffraction signals obtained after azimuthally averaging are shown in the graph. The difference between the diffraction data of the cell material and of the sample holder is depicted in the lower left corner. A slightly modified power-law yields an exponent of $\nu=-3.6$. In both graphs the points $q=q_{\dagger} \equiv(c / a)^{1 / \nu}$ are marked. 


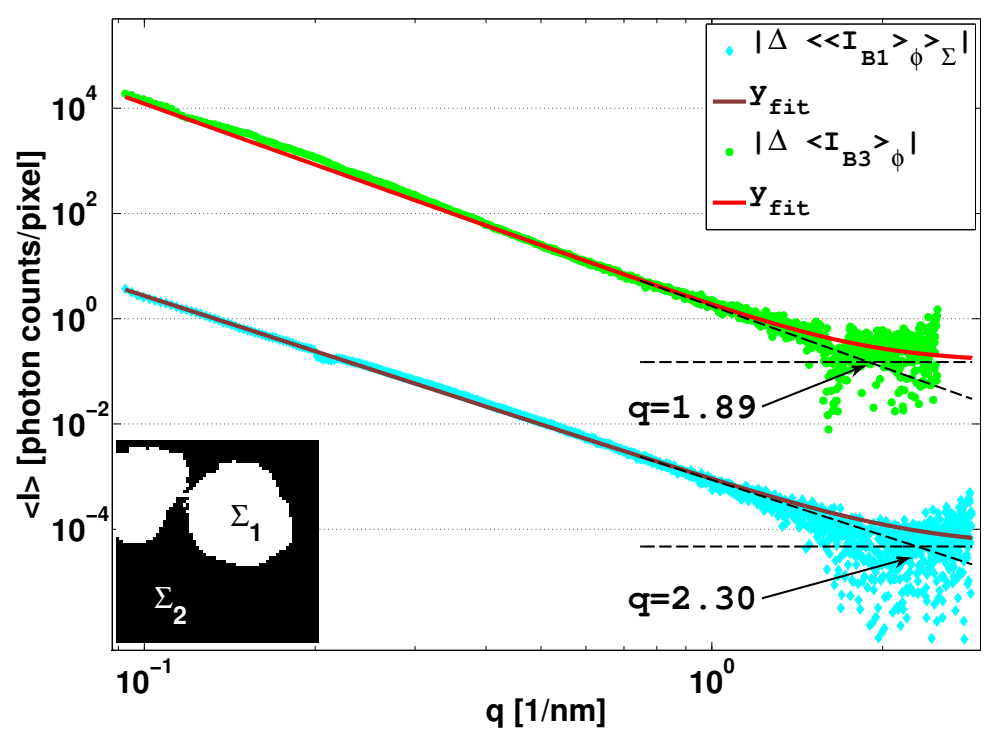

Figure 3.31: Sample region $\mathbf{B}$ - incoherent setting: incoherent diffraction curves were obtained from data $B_{1}$ and $B_{3}$ (cf. Table 3.7 ). The image segmentation of $B_{1}$ according to areas with cell signal, $\Sigma_{1}$, and without cell signal, $\Sigma_{2}$, is shown as an inset. Power-law fits yield exponents of $\nu=-3.5$ and $\nu=-3.8$ for $B_{1}$ and $B_{3}$, respectively. The points $q=q_{\dagger} \equiv(c / a)^{1 / \nu}$ are marked for both curves. 


\subsubsection{Details and Theory: Ptychographic Imaging}

First, the necessary nomenclature being used here is outlined briefly. Assuming that the exit surface wave $\Psi_{j}(\vec{\rho})$ (ESW) directly behind the sample can be formulated as a product of the illuminating wave-field $P\left(\vec{\rho}-\vec{\rho}_{j}\right)$ and the complex transmission of the specimen $O(\vec{\rho})$, a Fourier transform relation holds between the ESW and the intensity $I_{j}$ at a Fraunhofer or farfield detection plane

$$
I_{j}=\left|\mathcal{F}\left\{\Psi_{j}(\vec{\rho})\right\}\right|^{2}=\left|\mathcal{F}\left\{P\left(\vec{\rho}-\vec{\rho}_{j}\right) O(\vec{\rho})\right\}\right|^{2} .
$$

Ptychographic algorithms can be used to solve for $O(\vec{\rho})$ and $P(\vec{\rho})$ (cf. section 1.3.6, p. 40). In this experiment, a set of $j=1, \ldots, J$ diffraction patterns was collected by scanning the sample along concentric circles in a $X Y$-plane (cf. 62], Fig. 3.33(a)) allowing adjacent illuminated spots to overlap (influence of the overlap parameter see $[30 \mid)$. Usually, further issues have to be considered in the reconstruction scheme for Eq. (3.109) to hold: These are experimental uncertainties such as positioning errors due to the limited experimental accuracy, drifts and vibrations, noise in the recorded data, unusable areas of the detector, a limited stationarity of the probe and partial spatial coherence. In particular, phasing of weakly scattering specimens such as bacterial cells is a challenging task 62,97 .

Next, details about the algorithmic and experimental parameters are listed. Here, two different ptychographic algorithms were used: 'DM' (difference-map) 294 (and references therein) and 'ePIE' 183 (see also section 1.3.6, p. 40). Following the strategy of 62, a Siemens star resolution test chart (model ATN/ XRESO$50 \mathrm{HC}$, NTT-AT, Japan) was used in order to get a good estimate of the probe, which could be used as an initial guess for the phasing of the bacterial specimens. The sample was placed into a defocus position of $\sim 1 \mathrm{~mm} .323$ diffraction patterns have been measured using a scan pattern with scan points on concentric circles (cf. Fig. $3.33(\mathrm{a})$ ), a dwell time of $0.2 \mathrm{~s}$ or equivalently, an exposure time of $\simeq 1 \mathrm{~min}$ in total. The distance between adjacent concentric circles was $0.5 \mu \mathrm{m}$ in comparison to $\sim 0.75 \mu \mathrm{m} \times 1.5 \mu \mathrm{m}$ FWHM values of probe intensity, $\mathrm{h} \times \mathrm{v}$ respectively. A subset of $800 \times 800$ pixels of the recorded whole diffraction patterns (cf. Fig. $3.33(\mathrm{~b}))$ was used for phasing ( $\sim 10 \mathrm{~nm}$ real space pixel size). Dead or hot pixels of the detector were masked and their intensity values were defined by the current iterate in the reconstruction scheme. Moreover, the ptychographic reconstruction algorithm ('ePIE' algorithm) was initiated using the complex field of a $1 \mathrm{~mm}$ forward propagated, circular aperture with uniform amplitude and constant phase as probe function and uniform amplitude with constant phase as object guess. Additionally, the amplitude of the object was clipped onto the interval $[0.7,1]$ during each iteration. Note that the interval has been chosen close to the theoretical expectation. For a thickness of $T=500 \mathrm{~nm}$ tantalum (mass density $16.65 \mathrm{~g} / \mathrm{cm}^{3}$ ) one finds according to $[125]$ an amplitude attenuation of $\exp (-k \beta T)=0.8795$ and a phase shift of $\Delta \phi=-k \delta T=-1.078 \mathrm{rad}$ (cf. equation 1.69, p. 26). The final reconstruction (cf. Fig. 3.33(a)) was obtained after 320 iterations by averaging over the last 200 iterations with an average interval of 2 . Superior results are obtained by using the 'ePIE' algorithm with parameters $\alpha=\beta=1 / 2$ (cf. Eq. (6), 
(7) in $[183 \mid)$. The error curve of the reconstruction is presented in Fig. $3.32(\mathrm{a})$, In case of the ptychographic datasets that have been used to analyse the influence of the coherence defining slit gap S0 on the reconstructions (section 3.1.8), best results were obtained using the DM algorithm. Six datasets were collected, each consisting of 258 scan points using an exposure time of $0.2 \mathrm{~s}$ and a grid spacing of $0.28 \mu \mathrm{m}$ between adjacent concentric circles. The DM algorithm was run for 300 iterations and the final results were obtained as an average over every fifth iterate of the last 150 iterations. During each iteration the object was clipped onto the interval $[0.7,1]$. The probe was initialised with a reconstruction that was obtained at lower resolution beforehand.

The phase reconstructions of cells of $D$. radiodurans on the same sample, which was studied before by cellular nano-diffraction (cf. Fig. 3.42), were obtained using the 'DM' algorithm for the simple reason that it can be parallelised. Here, both algorithms yield reconstructions of comparable quality, as judged from single projections. The sample being mounted on a thin polyimide foil was moved to a defocus position of approximately $z_{01} \approx 1 \mathrm{~mm}$. 41 ptychographic projections were performed. The angular increment between successive projections is $\Delta \Phi=3.5^{\circ}$, leaving a missing wedge of $\pm 20^{\circ}$. Each ptychographic projection corresponds to 323 diffraction patterns, which have been taken on a grid of concentric circles using a dwell time of $0.2 \mathrm{~s}$. Including movements of motors, the task was completed after about $100 \mathrm{~min}$, i.e. $\sim 2.5 \mathrm{~min}$ per projection. A subset of $194 \times 194$ pixels of each PILATUS image has been chosen for the reconstructions, which corresponds to a real space pixel size of about $43 \mathrm{~nm}$. Importantly, the ptychographic reconstructions were initiated with the reconstructed illuminating function from the Siemens star as a probe guess and a uniform amplitude, constant phase object guess. During each iteration the amplitude of the object was clipped onto the interval $[0.9,1]$. The final objects (and probes) were obtained as an average over every second iterate of the last 100 of 200 iterations.

The phase reconstructions of $D$. radiodurans (cf. Fig. 3.43) being placed on a $\mathrm{Si}_{3} \mathrm{~N}_{4}$ membrane were obtained similarly as before. Two different regions with bacteria have been studied, Fig. $3.43(\mathrm{a})$ and Fig. $3.43(\mathrm{~b})-3.43(\mathrm{~d})$ respectively. The samples were measured in the focal region (probe size: $0.5 \mu \mathrm{m} \times 0.4 \mu \mathrm{m}$ full width at half maximum (FWHM) values of probe intensity, $\mathrm{h} \times \mathrm{v}$ respectively; see table 3.8 for scan details). 77 ptychographic datasets were recorded for the tomographic reconstruction of the cellular sample seen in Fig. 3.43(b). The angular increment between successive projections is $\Delta \Phi=2^{\circ}$, leaving a missing-wedge of $\pm 13^{\circ}$. In each projection a dwell time of $0.2 \mathrm{~s}$ was used. The grid spacing between adjacent concentric circles was $0.35 \mu \mathrm{m}$. Three projections could not be reconstructed and have been omitted for the tomographic reconstruction. To quantify the errors resulting from the missing data, the reconstruction was simulated based on a test phantom with parameters comparable to the experimental situation (cf. section D. p. 193). An upper limit of $10 \%$ error in the reconstructed density values was found. These errors were dominated by the missing-wedge, while the three missing projections were found to have a negligible effect. A subset of $192 \times 192$ pixels of each PILATUS image corresponding to a real space pixel size of $46 \mathrm{~nm}$ has 
been chosen for all reconstructions. Importantly, the ptychographic reconstructions were initiated with the reconstructed illuminating function from a Siemens star dataset (not shown) as a probe guess and a uniform amplitude, constant phase object guess.

In addition, another constraint was enforced onto the data of bacterial specimens. A rectangular support $\chi$ of the visible cell cluster was defined and during each iteration the phase outside the support was set close to zero using the following phase constraint:

$$
\mathcal{P}(O(\vec{x})):=\left\{\begin{array}{ll}
|O(\vec{x})| \cdot \exp (i \min (\operatorname{Arg}(O(\vec{x})), 0)) & \forall \vec{x} \in \chi \\
|O(\vec{x})| \cdot \exp (i \gamma \cdot \operatorname{Arg}(O(\vec{x}))) & \forall \vec{x} \notin \chi
\end{array},\right.
$$

where $\gamma \in \mathbb{R}$ was chosen close to zero. It should be noted that $\mathcal{P}(O(\vec{x}))$ enforces a negative phase shift on the object transmission. However, this represents no restriction since one usually is interested in the relative phase shift between different materials. Moreover, the object transmission can be easily adapted to cover the full phase range by multiplying $\mathcal{P}(O(\vec{x}))$ by $\exp (i \pi)$. The final results were obtained by averaging over 500 iterations during the so-called steady state of the 'DM' algorithm (cf. Fig. 3.32).

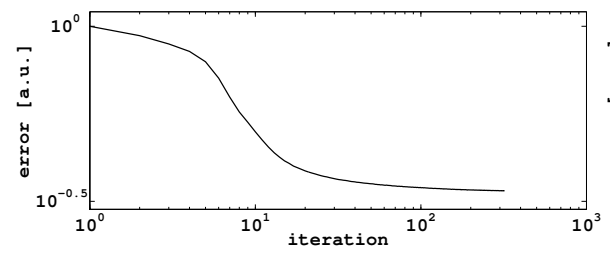

(a)

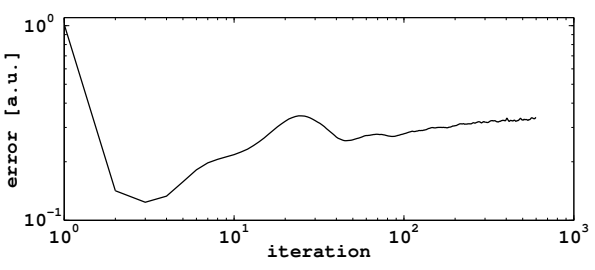

(b)

Figure 3.32: The figure shows different calculated error curves for ptychographic reconstructions. (a) presents the nomalized error curve according to equation 1.100 (p. 42). Note that the error quickly descends within 30 iterations. Afterwards the decrease of the error is slower. (b) presents the (normalized) 'DM-error' |294], which measures the deviation between the current exit surface waves $\Psi_{j}$ and the exit surface waves after application of the modulus constraint. After about 20 iterations, the 'DM' algorithm reaches a steady state where the error remains approximately constant.

For the interpretation of the ptychographic reconstructions, the 'phase retrieval transfer function' (PRTF) was used, which indicates the goodness of the reconstruction in comparison to the measured intensities (cf. [39, 97, 274], Fig. 3.34). Best results were obtained through the averaging procedure over many iterations and thereby cancelling randomly fluctuating phase contributions. Therefore, the closest solution for the measured intensities will be that corresponding to the exit surface waves $\left\langle\Psi_{j}(\vec{x})\right\rangle_{\text {it }}$ that have been averaged over many iterations. An overall estimate of the full ptychographic reconstruction can be achieved by averaging over all probe positions (corresponding to measured diffraction patterns $j$ ). In ad- 
dition, an azimuthal average was performed. The PRTF was thus used as defined by:

$$
\operatorname{PRTF}(|\vec{q}|)=\frac{\left\langle\left\langle\sqrt{I_{j}^{\mathrm{PCDI}}}\right\rangle_{j}\right\rangle_{\varphi}}{\left\langle\left\langle\sqrt{I_{j}^{\text {meas }}}\right\rangle_{j}\right\rangle_{\varphi}}=\frac{\left\langle\left\langle\left|\mathcal{F}\left\{\left\langle\Psi_{j}\right\rangle_{i t}\right\}\right|\right\rangle_{j}\right\rangle_{\varphi}}{\left\langle\left\langle\sqrt{I_{j}^{\text {meas }}}\right\rangle_{j}\right\rangle_{\varphi}}
$$

Additionally, it is convenient to analyse the resolution in terms of spatial frequencies by calculating the power spectral density (PSD), namely the modulus squared of the Fourier transform of any function. In order to be able to analyse the true power spectrum, it is often necessary to be careful with the numerical calculation of the Fourier transform, i.e. it involves the use of a window function. In case of the weak contrast images of phase reconstructions of D. radiodurans cells, the Kaiser-Bessel window has proven very helpful [95] (see also section 1.1.2, p. 12). The Kaiser-Bessel window (equation 1.21, p. 14) was also used here with parameters in the range $\beta \in[8,12]$. Results from different ptychographic phase reconstructions are collectively presented in figure 3.34 .

\subsubsection{Details: Tomographic Reconstruction}

At first, the ptychographically reconstructed projections (PCDI parameters for 2D reconstructions as above) have been aligned with respect to the axis of rotation. This can be done by calculating the cross-correlation between adjacent projections 113, which, however, may need manual corrections. Equal results have been obtained more quickly by using a centre of mass approach 270 in combination with the fact that the specimen here is isolated and that its support $\chi(x, y, z)$ can be determined from single projections. It should be recalled, that the tomographic reconstruction is carried out independently for different $\mathrm{z}$-slices (z: vertical direction) using the '2d'-Radon transform of a $3 \mathrm{~d}$ density function $f(x, y, z)$

$$
P_{\theta}(t, z)=\iiint_{\chi} f\left(x, y, z^{\prime}\right) \delta(x \cos \theta+y \sin \theta-t) \delta\left(z^{\prime}-z\right) d x d y d z^{\prime} .
$$

Now consider the centre of mass coordinates of the projections $M_{t, \theta}$ and $M_{z}$ :

$$
\begin{aligned}
M_{z} & :=\iint_{\chi} z P_{\theta}(t, z) d t d z \\
& =\int_{\chi} z f\left(x, y, z^{\prime}\right) \delta(x \cos \theta+y \sin \theta-t) \delta\left(z-z^{\prime}\right) d x d y d z^{\prime} d t d z \\
& =\iiint_{\chi} z f(x, y, z) d x d y d z \equiv R_{z},
\end{aligned}
$$




$$
\begin{aligned}
M_{t, \theta} & :=\iint_{\chi} t P_{\theta}(t, z) d t d z \\
& =\int_{\chi} t f\left(x, y, z^{\prime}\right) \delta(x \cos \theta+y \sin \theta-t) \delta\left(z-z^{\prime}\right) d x d y d z^{\prime} d t d z \\
& =\int_{\chi}(x \cos \theta+y \sin \theta) f(x, y, z) d x d y d z \\
& =\cos \theta R_{x}+\sin \theta R_{y},
\end{aligned}
$$

where $R_{x}, R_{y}, R_{z}$ denote the usual center of mass coordinates. In both calculations the delta functions have been eliminated by integration. Equation 3.113 suggests a natural alignment in the vertical direction by using the centre of mass coordinate in the vertical direction for every projection. In addition, there is also the possibility to set $M_{t, \theta}=0, \forall \theta$. According to equation 3.114 , this can only be fulfilled iff $R_{x}=R_{y}=0$. Hence, an alignment in the other directions is also achieved. In case of the reconstructed $2 \mathrm{~d}$ phase maps of bacterial cells it should be noted that the data is not ideally suited for a centre of mass based alignment due to phase offsets and possibly radiation induced phase deterioration. For this reason, it was found that the support $\chi$ of each projection yields better results than the phase map itself. Afterwards, the 3D tomographic reconstruction was done by applying the Filtered Backprojection using the standard 'Ram-Lak' or ramp filter 151. Visualisation was done with the 'Avizo'-Software (Avizo Fire 7.0.1, Visualization Science group, USA).

\subsubsection{Results: Ptychographic Imaging of the Resolution Chart and Wave-Field Analysis}

The ptychographic reconstruction of the $500 \mathrm{~nm}$ thick layer of tantalum suitably matches the corresponding theoretical phase shift of $\Delta \Phi(\mathrm{E}=6.2 \mathrm{keV})=-0.34 \pi$ and transmission of $I / I_{0}=0.77$ (cf. Fig. E.1, p. 195). Its innermost part consisting of $50 \mathrm{~nm}$ structures is clearly resolved (cf. Fig. 3.33). The 'overall'PRTF (cf. Eq. (3.111)) in Fig. 3.34(a) (green curve) shows signal at higher frequencies beyond the black dash-dotted line of $\nu=34 \mu \mathrm{m}^{-1}$ or equivalently of $\mathrm{dx}=15 \mathrm{~nm}$ half-period length. In addition, the PRTF is calculated according to an average over a single scan point (red curve). Remarkably, both curves do not drop below the typical cut-off of 0.5 . Note that the contribution of the probe to the diffraction patterns is confined to a small circular area up to $\nu_{\max } \approx 5 \mu \mathrm{m}^{-1}$. The disc-like image of the FZP and OSA can be clearly distinguished from the contribution of the Siemens star at high frequencies. The resolution can thus be attributed to the sample and not the probe.

Briefly, the imaging regime of the diffraction patterns is discussed. Using a Gaussian beam model, the radius of curvature of the probe $R(z)=z\left(1+\left(z / z_{0}\right)^{2}\right), z_{0}$ being the Rayleigh range, can be approximated by $R(z) \approx z$ at a distance of ca. $1 \mathrm{~mm}$ to the focus. Estimating the probe diameter to be $1-3 \mu \mathrm{m}$ one thus obtains Fresnel numbers $F$ in the range of $4 \leq F \leq 45$. Note that in this regime one may rather speak of ptychographic Fresnel coherent diffractive imaging (cf. $|316,334|)$. 
The relationship between the resolution and the highest angle at which signal is recorded may thus not be applicable due to the phase curvature of the illuminating probe (cf. Fig. 3.33(a)). As an alternative, it is made use of the power spectral density (PSD) and line scans at sharp edges to determine the resolution. The phase of the line sections is drawn in the graphs next to and above the $2 \mathrm{~d}$ phase reconstruction in Fig. 3.33(a). A fit of the vertical and horizontal edges to an error function yields line-spread functions with FWHM-values of $16 \mathrm{~nm}(\mathrm{v})$ and $17 \mathrm{~nm}(\mathrm{~h})$, respectively. In addition, the PSD of the reconstructed phase (cf. Fig. $3.34(\mathrm{~b}))$ supports the conclusion that features in the range corresponding to $10-15 \mathrm{~nm}$ half-period length are resolved.

A ptychographic reconstruction with respect to an object in a specific plane gives access to the entire beam by means of propagation. The reconstruction of the probe can thus be used to analyse the complex field of the focus $160,265,287$. Consider the following operation on the complex field of the probe $P$ ('sharpness', cf. 108 )

$$
\Upsilon(\Delta z):=\int_{\mathbb{R}^{2}} d S\left|\mathfrak{F}_{\mathfrak{R}}\{P\}(\vec{\rho}, \Delta z)\right|^{4},
$$

where $\mathfrak{F}_{\mathfrak{R}}\{\cdot\}(\cdot, \Delta z)$ denotes the free space back propagation by $\Delta z$ (cf. section 1.1 p. 7). Here, one determines the points of highest focusing by locating two maxima of $\Upsilon(\Delta z)$. The numerical back propagation of the complex wave-field thus reveals an astigmatism in the focal region ${ }^{18}$. The difference of propagation distances yields a distance of $\Delta F_{\mathrm{hv}} \simeq 0.7 \mathrm{~mm}$ between the two orthogonal directions of highest focusing. The field along the optical axis is displayed as planes of constant $\mathrm{x}$ and $\mathrm{y}$ through the centre (cf. Fig. 3.33(a)). A Gaussian fit to the regions of highest focusing yields FWHM-values of $89 \mathrm{~nm}$ in the vertical direction and $93 \mathrm{~nm}$ in the horizontal direction.

The following comparison between conventional knife-edge scans and the availability of reconstructed probe field was developed during the review process of 330 . It is also included here for completeness. Since beam properties can also be derived from measurements of caustics or multiple knife-edge scans, a brief comparison between the ptychographic approach and the conventional knife-edge approach shall be addressed here. To this end, it is assumed that the pixel size of the probe intensities and the step size of knife-edge scans is equal to the pixel size of the ptychographically reconstructed probe of $d x \approx 10 \mathrm{~nm}$. In other words, the practical implementation of knife-edge scans at the $10 \mathrm{~nm}$ range is not questioned. In mathematical terms a knife-edge scan along $x$ in a plane perpendicular to the optical axis spaced $\Delta z$ from a reference plane (e.g. focus plane) is defined as

$$
K(x, \Delta z)=\int_{-\infty}^{x} d x^{\prime} \int_{-\infty}^{\infty} d y\left|\mathfrak{F}_{\mathfrak{R}}\{P\}\left(x^{\prime}, y, \Delta z\right)\right|^{2},
$$

where $\mathfrak{F}_{\mathfrak{R}}\{P\}(x, y, \Delta z)$ denotes the free-space propagation of the probe $P$ by $\Delta z$. Beforehand, it is important to note that a reconstruction of the full probe inten-

\footnotetext{
${ }^{18} \mathrm{~A}$ video in AVI format of the propagated field can be downloaded from http://www. opticsinfobase.org/oe/viewmedia.cfm?uri=oe-20-17-19232\&seq=1
} 


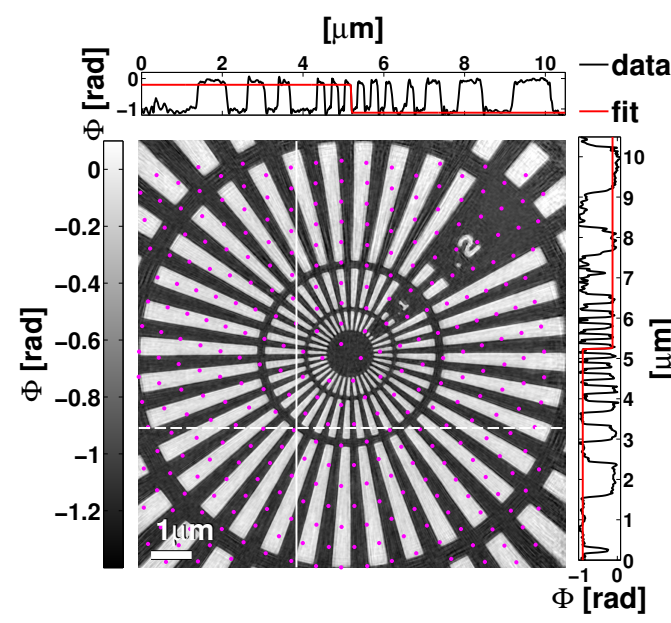

(a)

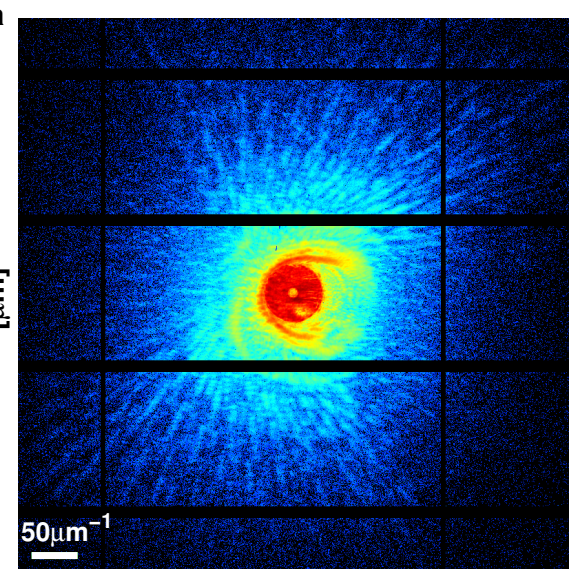

(b)

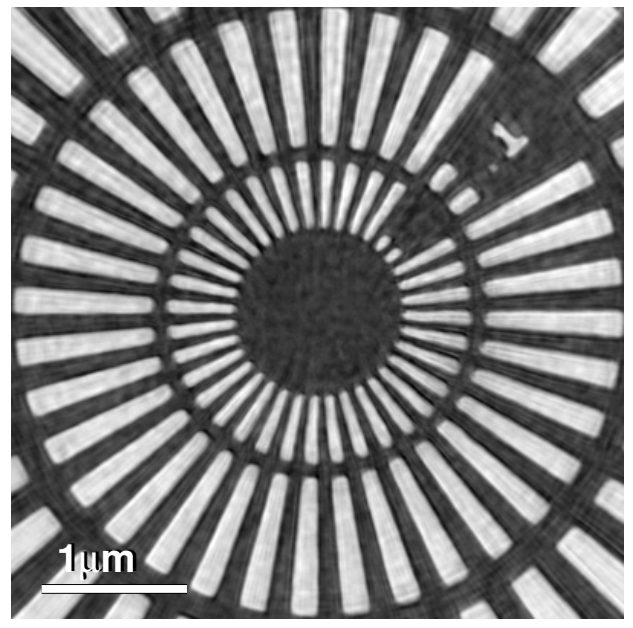

(c)

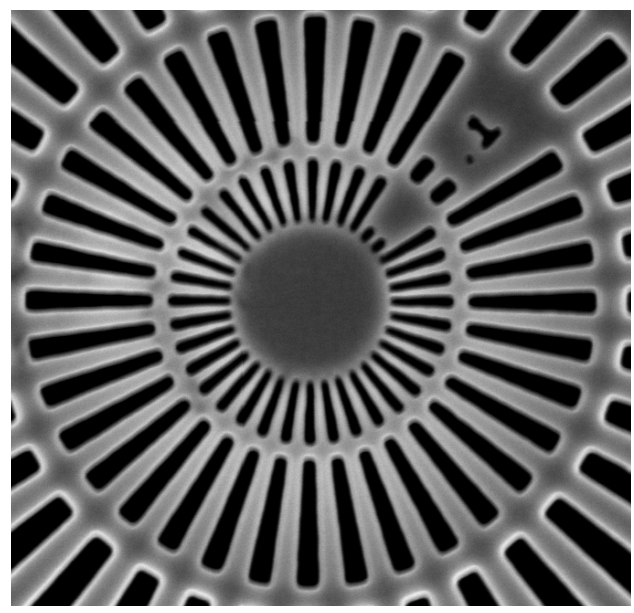

(d)

Figure 3.33: (a) Phase from 'ePIE' reconstruction of the tantalum test structure. Scan points are indicated as magenta points. A fit of the error function to one of the edges of the line scans yields a line-spread function with FWHM-values of $16 \mathrm{~nm}$ in the vertical direction and $17 \mathrm{~nm}$ in the horizontal direction. (b) A typical diffraction pattern of the PCDI dataset (log-scale) is shown. The scale bar corresponds to qvector. The inner part of (a) is shown in (c), (d) SEM image of the part of the X-ray resolution chart as presented in (c) (the SEM image is by courtesy of Mike Kanbach). 


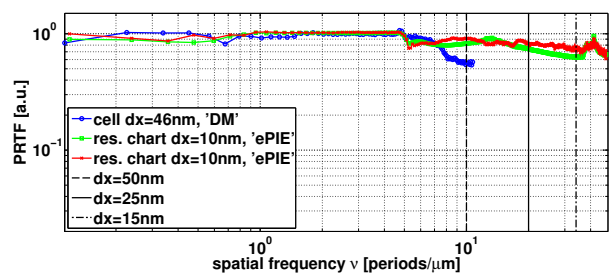

(a)

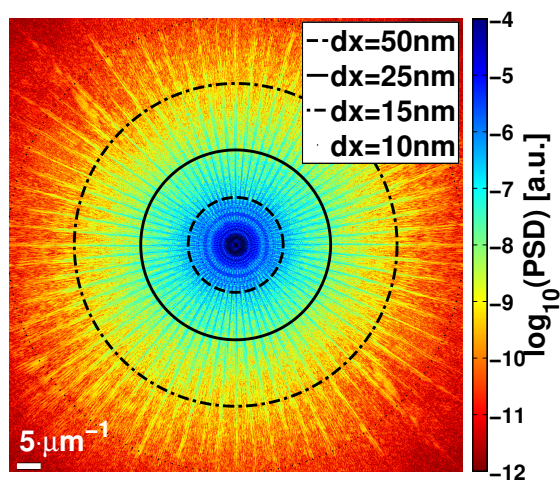

(c)

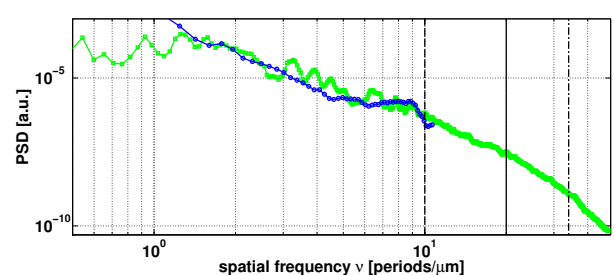

(b)

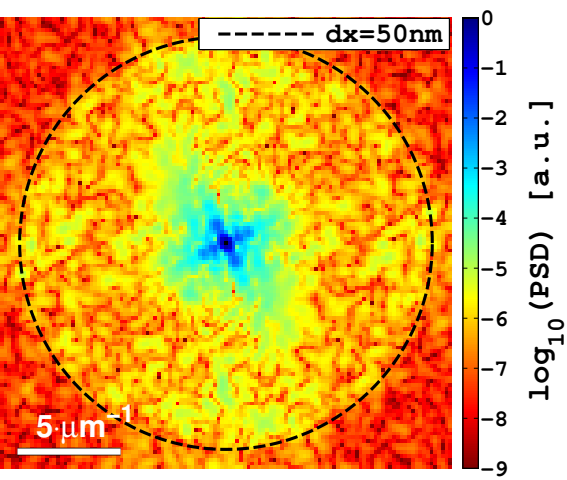

(d)

Figure 3.34: The PRTFs of the ptychographic reconstruction of the resolution test chart and of a single projection of cells (Fig. 3.43(b)) are depicted in (a) green curve and blue curve respectively. In addition, the PRTF of the test sample according to an average over a single scan point is shown as red curve (cf. Eq. (3.111)). The high frequency part of the spectrum of the azimuthally averaged power spectral densities (PSD) of the reconstructed phase distributions are displayed in (b). Real space half-periods of $50 \mathrm{~nm}, 25 \mathrm{~nm}$ and $15 \mathrm{~nm}$ are indicated as dashed, solid and dasheddotted black lines, respectively. Legend of (b) is legend in (a). (c) 2D PSD of 'ePIE' reconstruction of Siemens star test pattern. Note that the Siemens star structure can be clearly seen in the radial direction. (d) 2D PSD of ptychographic ('DM') reconstruction of $D$. radiodurans in Fig. 3.43(b) Scale bars correspond to spatial frequencies. 


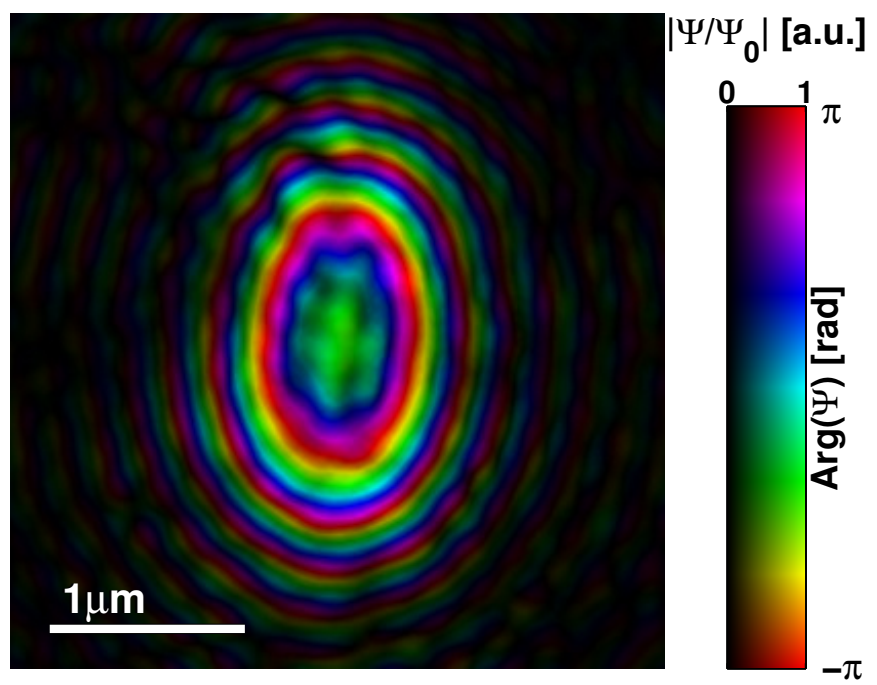

(a)
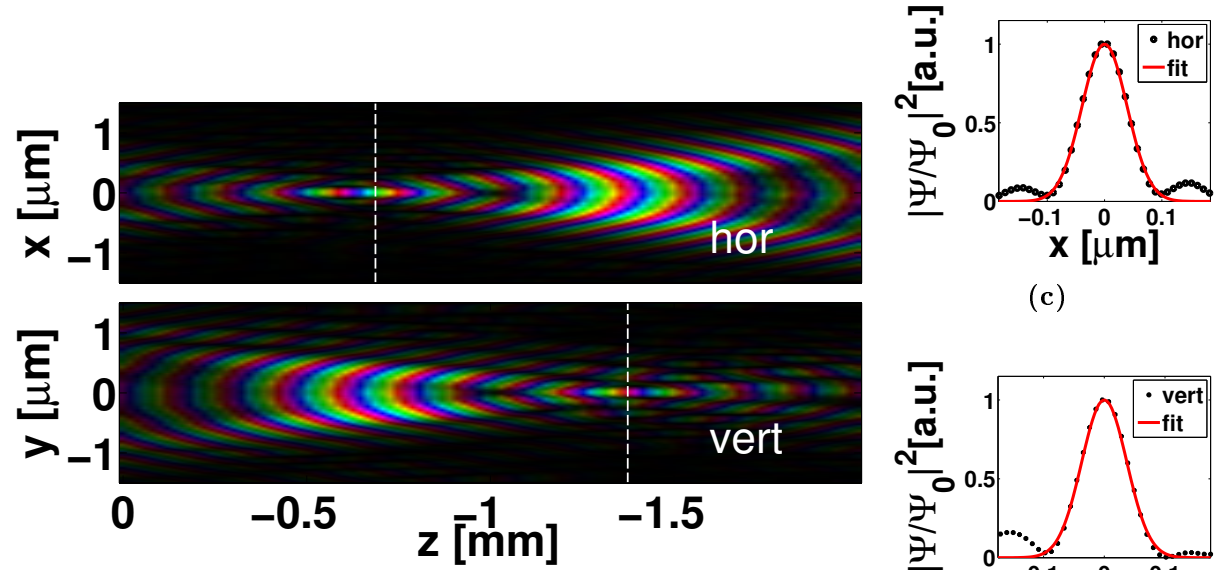

(b)

(c)

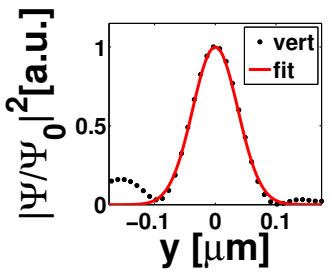

(d)

Figure 3.35: (a) Complex representation of reconstructed probe at the plane of the resolution chart sample (cf. Fig. 3.33). (b) horizontal and vertical line scans through the centre of the back-propagated probe (cf. footnote on page 84). The white dashed lines highlight the positions of different focal positions. (c) (d) Gaussian fits (solid red lines) to the peaks of the line scans of the probe intentsity are depicted together with the data from the line scans (black dots). FWHM-values of $87 \mathrm{~nm}$ in the vertical direction and $93 \mathrm{~nm}$ in the horizontal direction are obtained, respectively. 
sity $\left|\mathfrak{F}_{\mathfrak{R}}\{P\}\left(x^{\prime}, y, \Delta z\right)\right|^{2}$ or any function $f(x, y)$ from $K(x)$ is a analogous to $2 \mathrm{D}$ tomography. The derivative of $K(x)$ with respect to $x$ yields the line integral $\mathfrak{P}_{\alpha=0}(x)$ for a constant angle (here $\alpha=0$ ), which is known as Radon transform ${ }^{19}$ (see also [324])

$$
\partial_{x} K(x)=\int_{-\infty}^{\infty}\left|\mathfrak{F}_{\mathfrak{R}}\{P\}(x, y, \Delta z)\right|^{2} d y \equiv \mathfrak{P}_{\alpha=0}(x) .
$$

$\left|\mathfrak{F}_{\mathfrak{R}}\{P\}(x, y, \Delta z)\right|^{2}$ or any function $f(x, y) \in \mathbb{R}$ can thus be reconstructed if the knife-edge scan is carried out for a sufficient number of orientations of the knifeedge. Since this is obviously not possible for practical reasons, conventional knifeedge scans in two orthogonal directions is based on the assumption that the probe intensity is separable in the $x$ - and $y$-direction. With the popular assumption of a normal intensity distribution, the knife-edge integral is expressed in terms of the error function

$$
\begin{aligned}
\left|\mathfrak{F}_{\mathfrak{R}}\{P\}\left(x^{\prime}, y, \Delta z\right)\right|^{2} & =\exp \left(-(x-\mu)^{2} /\left(2 \sigma^{2}\right)\right) /(\sigma \sqrt{2 \pi}) \cdot f(y) \\
\Rightarrow K(x, \Delta z) & =\frac{C_{0}}{2}\left(1+\operatorname{erf}\left(\frac{x-\mu}{2 \sigma}\right)\right)
\end{aligned}
$$

where $C_{0}$ denotes a constant that arises from the integration along the $y$-direction. In this case a fit of $K(x, \Delta z)$ to the measured data yields the width of the beam, namely $\mathrm{FWHM}_{x}=2 \sigma \sqrt{2 \log 2}$.

At first, a simulated 'dumbbell-shaped' probe intensity (cf. Fig. 3.36(a)) is considered, which violates the assumption of factorization. Certainly, this modelprobe may have pronounced and somewhat exaggerated side-lobes. However, the reader may note that the probe reconstructed experimentally in this work also exhibits side-lobes at least in the plane shown in Fig. 3.36(d). For this choice of probe, the fit of the error function to the calculated knife-edge scan along the horizontal direction (cf. Fig. 3.36(b)) yields FWHM $=0.635 \mu \mathrm{m}$ in comparison to the theoretical value of FWHM $=0.1 \mu \mathrm{m}$ at the centre (cf. Fig. 3.36(c)).

Next, the experimental case of the reconstructed probe field intensity (ptychographic reconstruction of the Siemens star resolution chart, cf. Fig. (3.33) is considered. After a calculation of the probe at several planes behind the sample, 'numerical' knife-edge scans along the horizontal and vertical direction in a few hundred planes is carried out to obtain the beam-width along the optical axis. The horizontal and vertical beam-widths as a function of the distance to the sample plane are shown in Fig. 3.36(f) as red and blue curves, respectively. Note, that the minimal beam-widths do not exactly coincide with the maxima of $\Upsilon(\Delta z)$ (Eq. (3.115)), indicating that the horizontal and vertical axes do not coincide with the minor and major axes of the beam. The discrepancy increases even further if the probe is rotated. In Fig. 3.36(f) the magenta and green curves show the FWHM values in the horizontal and vertical direction determined by knife-edge scans of the probe after a rotation by $45^{\circ}$, respectively. In this case, the knife-edge

\footnotetext{
${ }^{19} \mathrm{~A}$ definition of the Radon transform is given in equation $1.101, \mathrm{p} .43$.
} 
scans are not sensitive to the astigmatism of the beam. As is known from knifeedge scanning of astigmatic Gaussian beams 23 , it is not generally sufficient to perform knife-edge scans along the horizontal and vertical axes. Contrarily, one needs to scan at several angles to determine the major and minor axes in one plane. Furthermore, it becomes clear that features such as the double-peak and donut-shape (at certain positions of the optical axis, cf. Fig. 3.29 (C)), will be extremely difficult to infer from a set of knife-edge scans along two orthogonal axes.

Finally, the intensity of the probe at $\Delta z=-0.686 \mathrm{~mm}$ (cf. Fig. $3.36(\mathrm{~d})$ ) in front of the plane of the sample is considered. In order to get an FWHM estimate that becomes close to the theoretical value of FWHM $=0.093 \mu \mathrm{m}$ (cf. Fig. 3.33) the fit of the error function to the knife-edge scan needs to be restricted to values close to the centre yielding an FWHM $=0.104 \mu \mathrm{m}$ (cf. Fig. 3.36(e)). The outcome of the FWHM value of the fit could also be improved by manually weighting the data points near the centre. However, without a two-dimensional representation of the probe intensity, justification for both means would not be granted. It can be concluded that even if the information on the phase front of the beam is not needed, reconstruction of the intensity distribution from selected knifeedge scans is problematic and does not stand up to the full probe reconstruction by ptychography. Therefore, it is recommend that ptychography should replace knife-edge scans whenever possible, i.e. if the beam is sufficiently coherent.

The complex-valued illumination field (probe) at the sample position is also useful to design, analyse and to control nano-diffraction experiments. As in many other optical applications, beam properties (focus position, size, depth of focus, aberrations) need to be known in order to perform controlled diffraction experiments, or more specifically nano-beam small-angle scattering experiments. Moreover, the knowledge of the probe can be used to simulate experimental images and to design an experiment (defocus, step sizes, convolution effects and dose expectations) as shown in Fig. 3.37, where the differential phase contrast of a model cell sample has been simulated for different positions along the optical axis using the experimental probe function. Here, the effect of a double peak in the illumination function on the differential phase contrast can be seen. That is, the sample appears twice in the image as can be seen in Fig. 3.37 (b).

The reconstructed probes $P$ from the ptychographic reconstructions were also used to investigate the applied surface doses $D$ of the different scanning experiments. For the case of dose sensitive biological material, which at the same time necessitates high doses of $\mathrm{X}$-ray radiation, it is quite important to determine the applied dose as best as possible in order to assess radiation damage effects. In a first step, the $2 \mathrm{D}$-photon density $\rho_{\gamma}(\vec{x})$ was calculated by scaling the normalized intensity of the probe at each scan position $j$ to the actual photon number $N_{j}$ : $\rho_{\gamma}(\vec{x})=\sum_{j} N_{j}\left|P\left(\vec{x}-\vec{x}_{j}\right)\right|^{2} / \int_{\mathbb{R}^{2}} d S|P(\vec{x})|^{2}$. Here, the reconstructed probe field gives access to the photon distribution on the scale of the resolution of the reconstruction. The results of different scans are presented in Fig. 3.38. The images reveal that in most cases the applied surface photon density is approximately constant, which justifies to characterise the applied dose by a single value. However, 


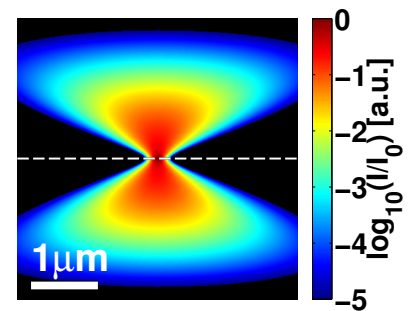

(a)

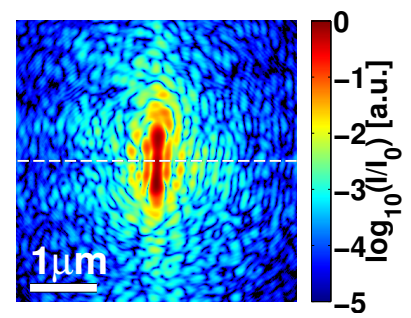

(d)

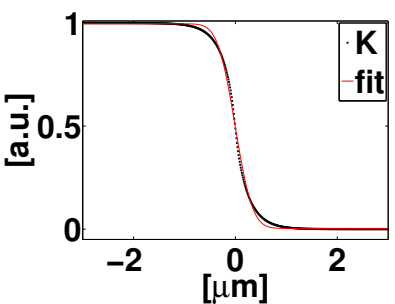

(b)

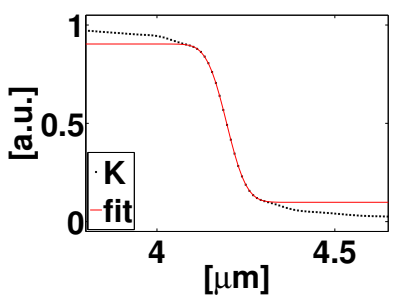

(e)

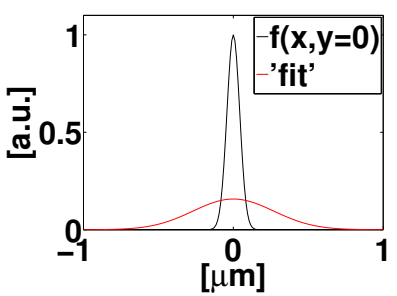

(c)

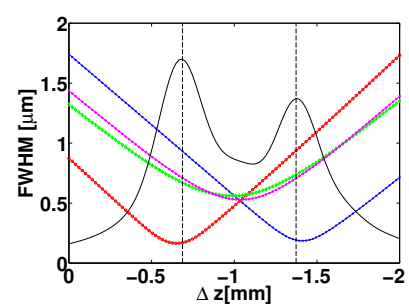

(f)

Figure 3.36: (a) Intensity of a model-probe exhibiting a dumbbell-shape. The calculated knife-edge scan along the horizontal direction is shown in (b) The white, dashed line in (a) indicates the region of highest focusing which is shown in (c) (solid, black line). The resulting Gaussian shape as estimated from the knife-edge scan is depicted as solid, red curve. (d) shows the intensity of the reconstructed probe at $\Delta z=-0.686 \mathrm{~mm}$ behind the plane of the sample. A white, dashed line indicates the part which has been used for estimating the beam width. The corresponding result of the calculated knife-edge scan is shown in (e). The FWHM values from calculated knife-edge scans are shown as function of distance to the sample plane in (f) The red and the blue curve denote horizontal and vertical beam-width, respectively. The magenta and green curves show the FWHM values in the horizontal and vertical direction determined by knife-edge scans of the probe after a rotation by $45^{\circ}$, respectively. In addition, $\Upsilon(\Delta z)$ is shown as solid, black curve which has been scaled arbitrarily for reasons of visualisation. 
(a)

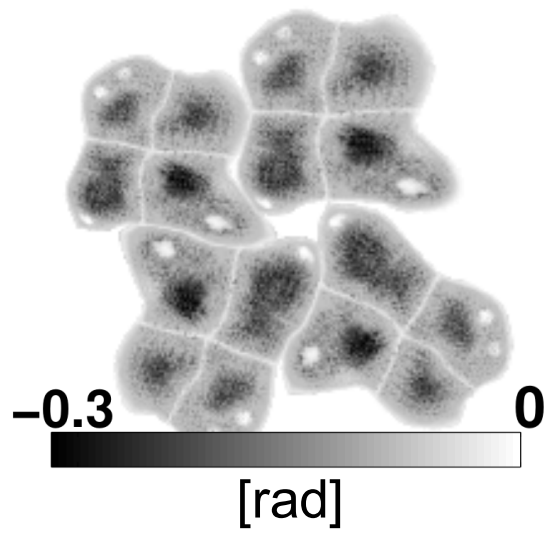

(c)

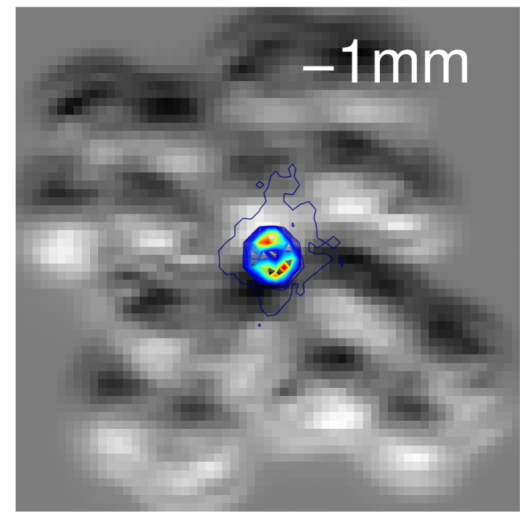

(b)

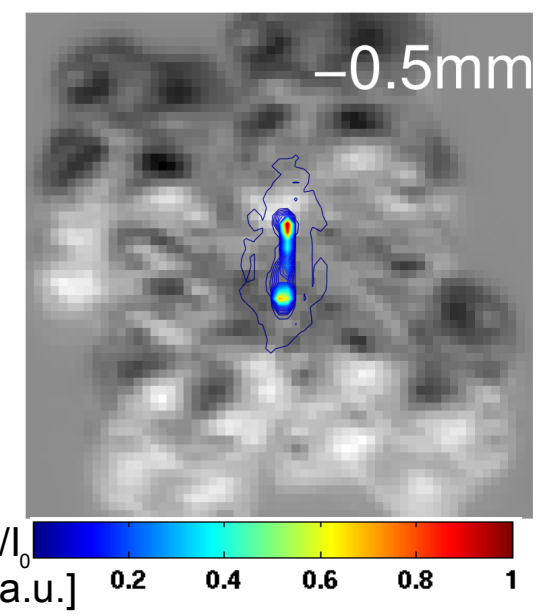

(d)

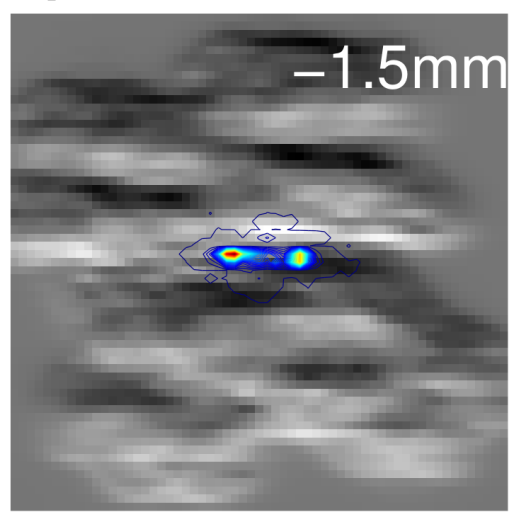

Figure 3.37: Numerical simulations: (a) synthetic cell sample and calculated differential phase contrast images (b)-(d) according to Eq. (3.109) using the back propagated probe of the Siemens star dataset (cf. Fig. 3.35) at different positions on the optical axis. For simplicity, only the vertical component of the differential phase contrast is shown. The distance of propagation with respect to the plane of the Siemens star sample is displayed and the corresponding intensity of the probe is visualised as an overlay. The colourcode of the probe intensities is according to the colourbar in (b). 
an inhomogeneous substructure becomes apparent if there is little or no overlap between adjacent illuminated spots (cf. Fig. 3.38(b)). Given the characteristic function $\chi(\vec{x})$ of a certain part of the cell area of the scan, the photon energy $E$, the mass density of the cell material $\rho_{c}$ and the absorption coefficient $\mu$, one obtains the applied surface dose $D[141$ of this region:

$$
D=\frac{\mu E}{\rho_{c}} \cdot \frac{\int_{\mathbb{R}^{2}} d S \cdot \chi(\vec{x}) \cdot \rho_{\gamma}(\vec{x})}{\int_{\mathbb{R}^{2}} d S \cdot \chi(\vec{x})} .
$$

All dose calculations are based on the assumption that the main composition of the cell material is protein of the empirical formula $\mathrm{H}_{50} \mathrm{C}_{30} \mathrm{~N}_{9} \mathrm{O}_{10} \mathrm{~S}_{1}$ of density $\rho_{c}=1.35 \mathrm{~g} / \mathrm{cm}^{3} \mid 141$. Results of the dose calculations are summarized in table 3.7 (p. 75) and table 3.8 (p. 101). 


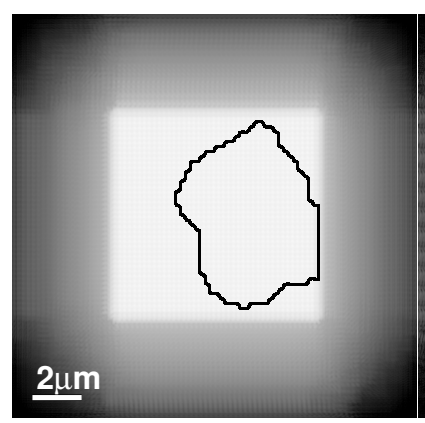

(a)

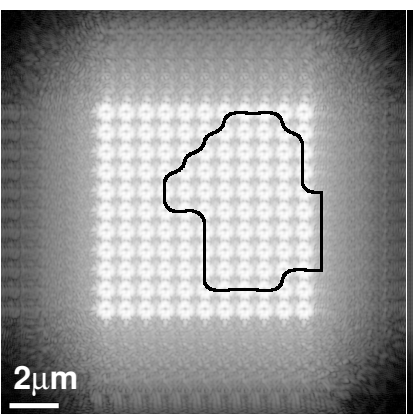

(b)

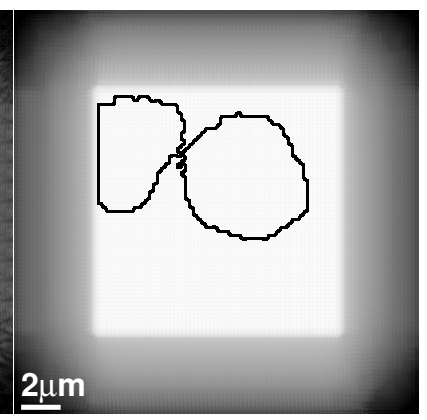

(c)

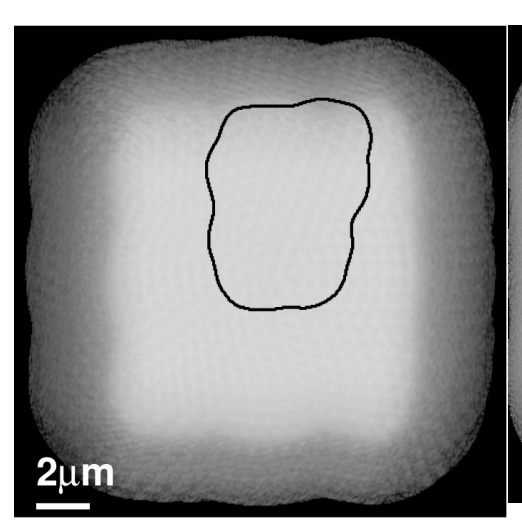

(d)

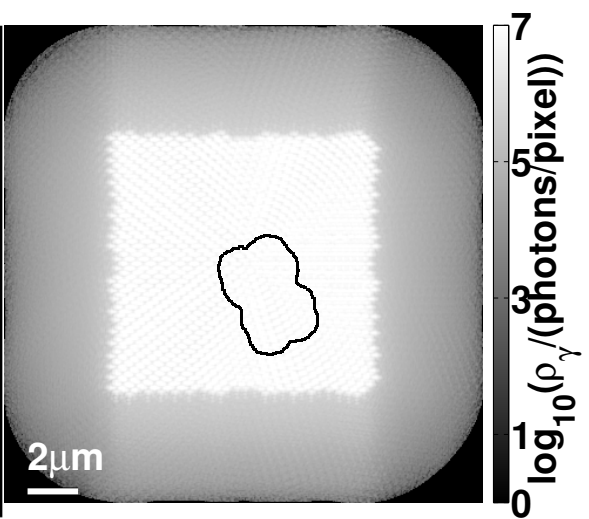

(e)

Figure 3.38: Photon surface densities $\rho_{\gamma}$ of diffraction datasets $A_{2}$ (a) $A_{3}$ (b) $B_{1}$ (c) and ptychographic datasets (d) (Fig. 3.41) and (e) (Fig. 3.43(a)). The location of bacterial cells is indicated by black lines. The central area which was scanned by the beam can be identified by a significantly higher photon surface density in all images. It can also be seen, that the tails of the beam illuminate a certain area outside this central region. Note that the difference in applied photon surface densities between the two ptychographic datasets, i.e. the fluence (dose), is lower in (d) than in (e) (a). (d) same colourbar as in (e) 


\subsubsection{Resolution vs. Coherence}

The coherence properties of the beam are primarily altered by the slit $\mathrm{S} 0$ in the horizontal direction. For instance in section 1.1.3 (p. 15), the coherence length of the beam at the slit S0 was estimated to be $6 \mu \mathrm{m}$ (cf. Tab 1.1, p. 19). In this section the influence of the gap width of the slit S0 on the ptychographic reconstructions is analysed. For this reason, a sequence of ptychographic datasets were recorded using the same parameters but with increasing slit gaps $\Delta x$. Starting from a nominal gap width of about $30 \mu \mathrm{m}$, it was increased in steps of $12 \mu \mathrm{m}$. Figure 3.39a shows ptychographic phase reconstructions of the Siemens star test pattern. As can be seen on the images (1)-(6) of figure 3.39a, the consequence of increasing the gap (decreasing coherence properties of the beam) is a decrease in resolution in the horizontal direction from about $100 \mathrm{~nm}$ (1) to above $200 \mathrm{~nm}$ (cf. white arrow heads). At the same time the photon flux increases linearly from $\sim 4 \cdot 10^{8}$ phot $/ \mathrm{s}$ to about $10^{9}$ phot $/ \mathrm{s}$ (Fig. 3.39b). Notably, the phase contrast appears to be in agreement to the resolution, i.e. the phase contrast also seems to remain quantitative. The reconstructions of the probe function (illuminating wave-field) are presented in Fig. 3.40a. At a first glance, the intensity distributions look remarkably similar at all gap positions. Fitting a Gaussian to the central line-cuts of the intensity distribution reveals a change of the beam size. In the vertical direction a moderate change from $280 \mathrm{~nm}$ (FWHM) to $226 \mathrm{~nm}$ (FWHM) is determined (cf. Fig. 3.40b). The decrease in size in the horizontal direction is determined to be more signficant. That is, increasing the gap width by $60 \mu \mathrm{m}$ yields a decrease in size from $470 \mathrm{~nm}$ (FWHM) to $370 \mathrm{~nm}$ (FWHM). The slit S0 acts as the beam defining slit. It determines the numerical aperture of the Fresnel zone plate. Hence, a decrease of the focal size in the horizontal direction is in agreement to the expectation.

In conclusion, the ptychographic reconstructions appear to be remarkably robust with respect to the considered gap widths which are quite large in comparison to the theoretical optimum value of about $6 \mu \mathrm{m}$. Nevertheless, it is shown how the maximum achievable resolution of the reconstruction can be increased by using less photons which are spatially more coherent. 


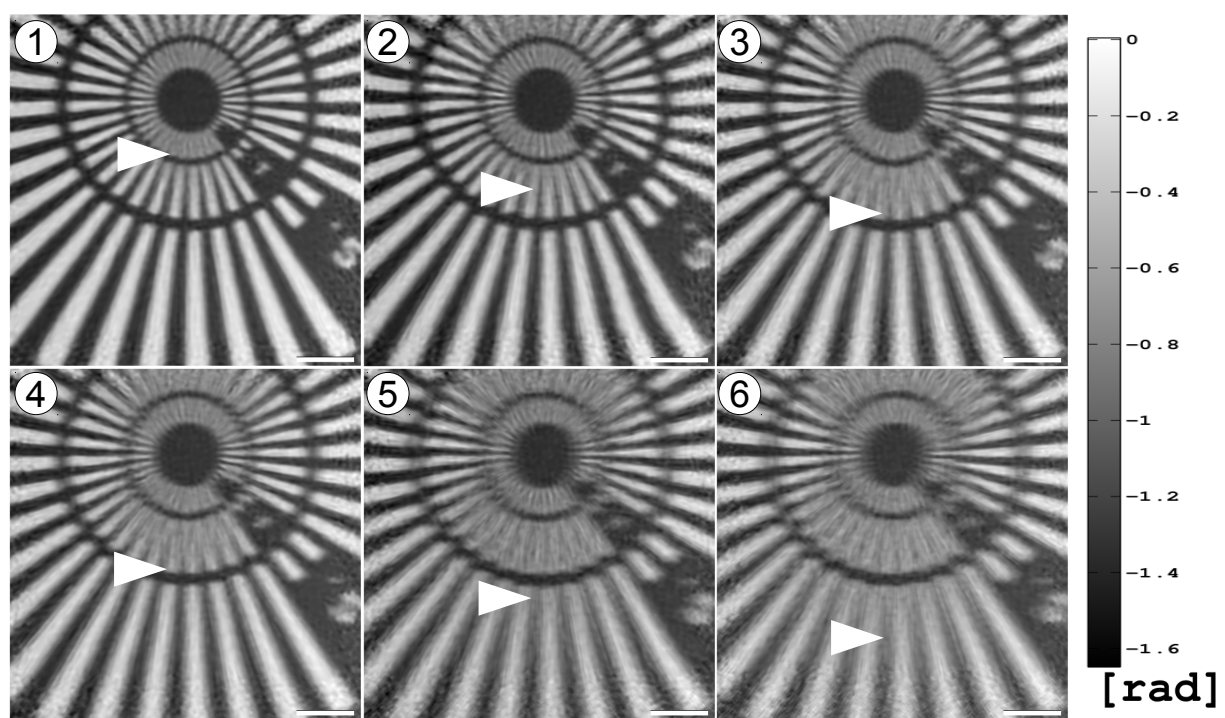

(a)

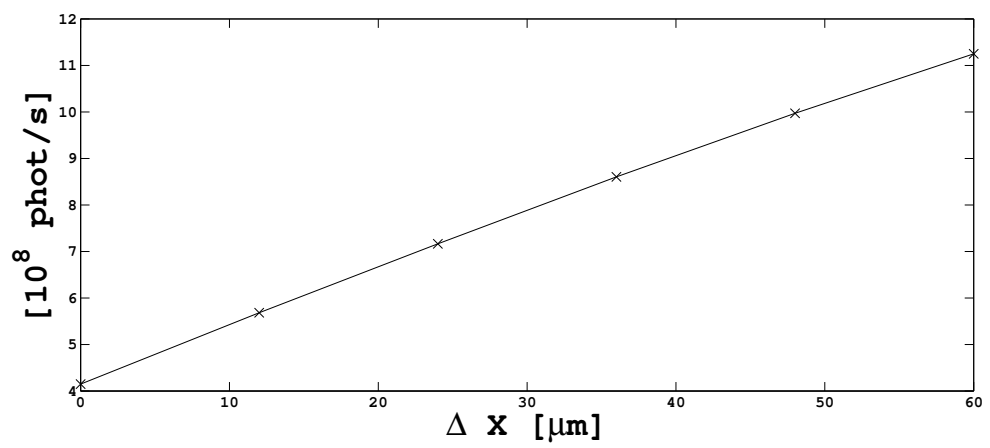

(b)

Figure 3.39: (a) shows the phases from ptychographic reconstructions of the X-ray resolution chart taken at different slit S0 gaps. The gap width $\Delta x$ is increased in steps of $12 \mu \mathrm{m}$ from reconstruction (1) to (6). The white arrow heads indicate the decrease in resolution in the horizontal direction. (b) illustrates the increase in photon flux that accompanies the increase in the gap width at the six positions. The color code is the same for all reconstructions in (a). The scale bars denote $1 \mu \mathrm{m}$. 


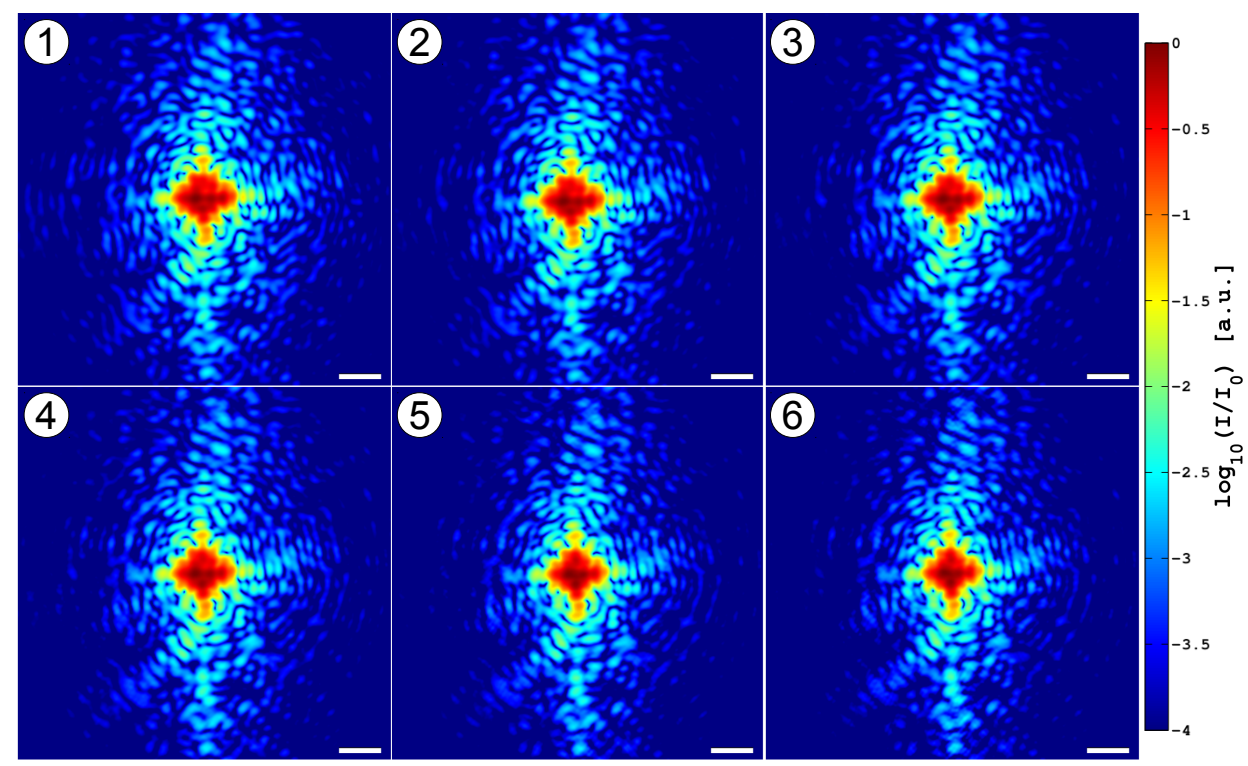

(a)

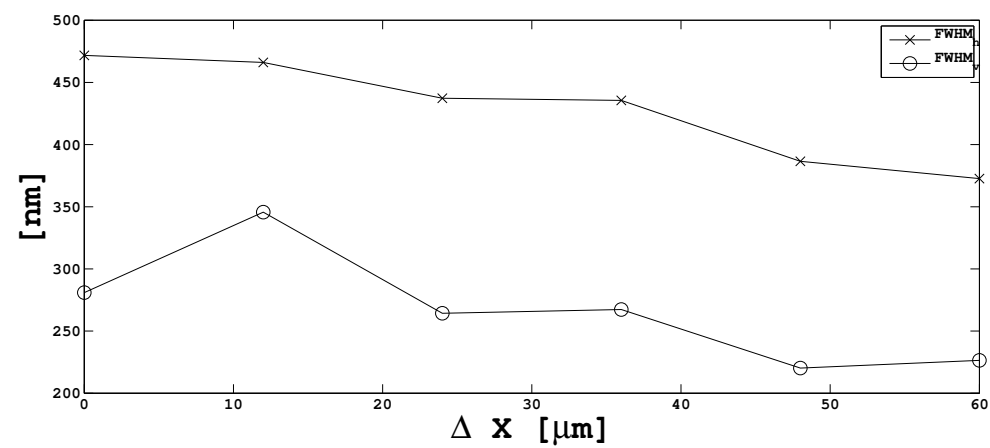

(b)

Figure 3.40: (a) presents the reconstructed probe intensities corresponding to the reconstructions shown in Fig. 3.39a. (b) shows fit results of a Gaussian distribution to the beam width in the horizontal and vertical directions. The results are given in terms of FWHM values as a function of the increase in the slit gap $\Delta x$ of the slit S0. A decrease in size of the probe at the sample is observed in both directions but the horizontal direction is affected more strongly. Here, the decrease in size is by about $100 \mathrm{~nm}$. 


\subsubsection{Results: Ptychographic Imaging Towards a 3D Representation of D. radiodurans Cells}

The first ptychographic imaging experiment on freeze-dried, unstained bacterial cells $D$. radiodurans of the same sample that has been analysed by cellular nanodiffraction reveals an image of a large cluster of bacterial cells in a single projection (Fig. 3.41). From the fact that the cells form an aggregate of many cells, one may deduce that these cells could have been in a metabolically active state of growth before plunge-freezing. A prominent feature of the cells is visible as electron density depleted regions (negligible phase shift), which appear as a granular structure with a broad size distribution. As will become clear, when discussing the full set of projections below, this feature is likely an artefact from radiation damage. Nevertheless, the cell structure may be still well preserved in parts of the sample. For instance, some of the $D$. radiodurans cells occur in their famous tetrad form. The location of two tetrads is indicated by a dashed, grey frame. Here, the granular structure is less dominant and the cells can be clearly identified. Notably, the resolution over the whole sample appears to be high enough to resolve possibly the cell walls which are indicated by a black arrowhead. With respect to the full sample, one finds features that are in the range of the pixel dimension indicating that the resolution is in the same range $(43 \mathrm{~nm})$. An average fluence of about $3.1 \cdot 10^{8}$ photons $/ \mu \mathrm{m}^{2}$ (cf. Fig. 3.38) yields an applied surface dose of about $6.7 \cdot 10^{5} \mathrm{~Gy}$.

The angular tilt series of ptychographic reconstructions with an angular spacing of $3.5^{\circ}$ is presented in figure $3.42(\mathrm{a})$. The images give a good impression of the overall 3d structure of the cellular aggregate. The reconstruction quality of the projections no. 32 and 33 is slightly reduced because the sample was drifting out of the scanned area. At the border of the illuminated area the sample was illuminated with a reduced fluence. A reduction in contrast is the consequence. Following the time series from projection 1 to 41, it can be deduced that the granular 'holes' must be induced by radiation damage as they partly grow with time, i.e. dose, or appear and disappear as indicated by the black arrows. The origin of these features can not be fully explained but it may be an artefact due to the removal of the cryojet, that was present during the diffraction experiment, in order to achieve the stability requirements for ptychographic phasing. An increase in humidity of the sample may have led to bubble formation during exposure to the intense synchrotron beam, which represents a challenge for experiments on hydrated samples (cf. $[322$ ). Surprisingly, these projections are still sufficient to yield an approximate $3 \mathrm{~d}$ representation of the sample as can be seen in figure 3.42 (b). On the image, the cells are visualised by showing three identical tomographic reconstructions from three different angular perspectives. Besides the overall shape, some cell compartments can be clearly identified. For instance, this is best seen in the region of the tetrads at the top of the sample. 


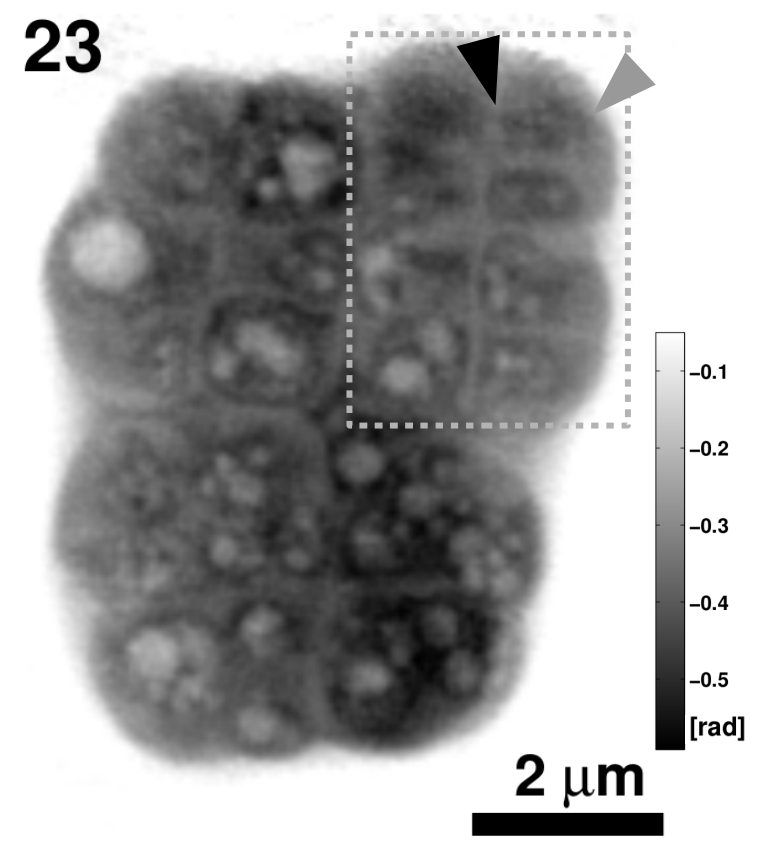

Figure 3.41: Phase from 'DM' reconstruction of single projection of $D$. radiodurans cells on a polyimide foil. The dashed grey frame indicates the region of two tedrads of cells. The grey arrowhead indicates inner cellular material whereas the black arrow points onto a cell wall. The number represents a link to the projections in Fig. 3.42 (a). 


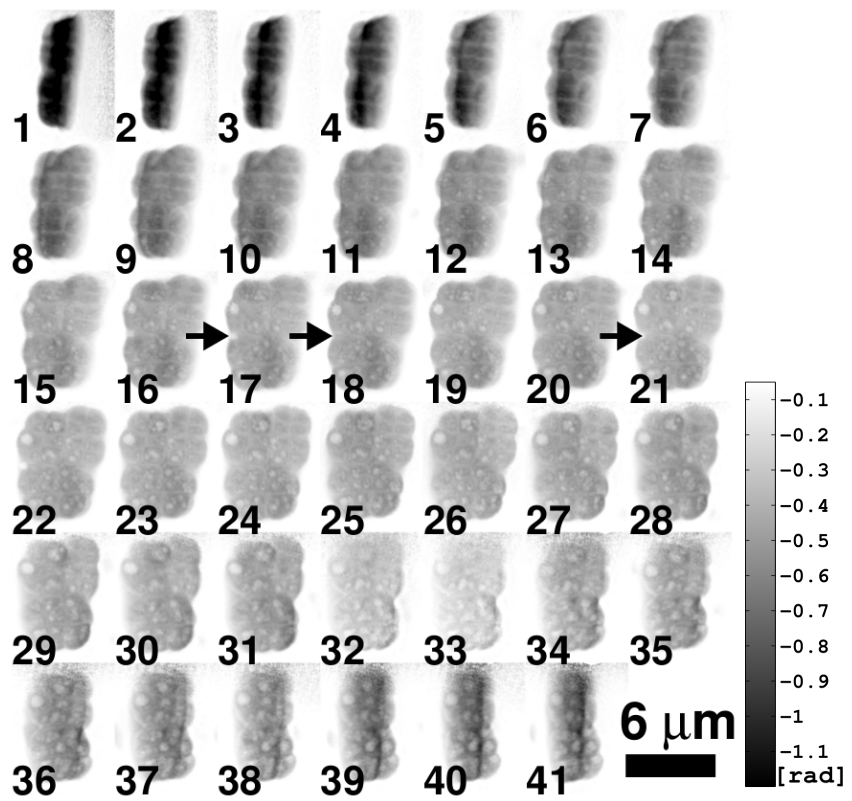

(a)

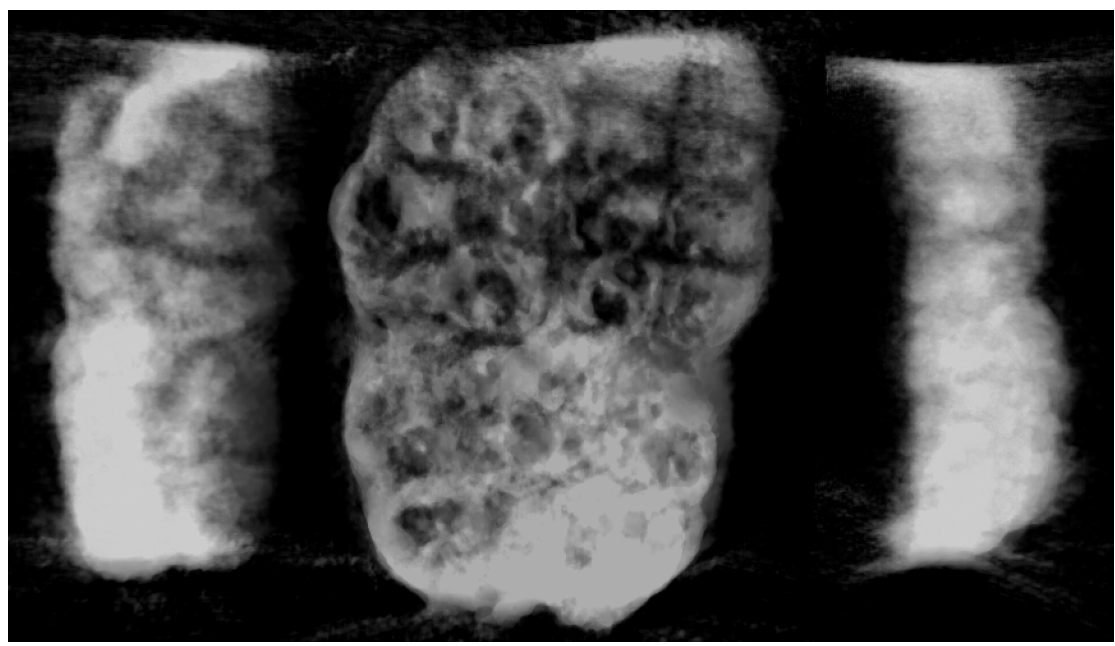

(b)

Figure 3.42: (a) shows phase reconstructions of $D$. radiodurans cells from different projection angles. The angular increment is $\Delta \Phi=3.5^{\circ}$ between successive projections. The arrows indicate the position of appearing and disappearing 'bubbles' (electron density depleted region). The numbers in (a) enumerate the projections. (b) presents an image of the $3 \mathrm{D}$ visualisation of the tomographic reconstruction of the set of projection images in (a) The visualisation shows the agglomeration of bacterial cells from three different angles. 


\subsubsection{Results: Tomo-Ptychographic Imaging of $D$. radiodurans cells}

This section is dedicated to the final (improved) three-dimensional reconstruction of unsliced, unstained, freeze-dried cells of D. radiodurans as presented in 330 . The results of the ptychographic reconstructions are presented in Fig. 3.43 . In Fig. $3.43(\mathrm{a})$ the typical tetrad form of $D$. radiodurans cells can be seen. Extending previous results [97], one can clearly identify disc-like regions exhibiting a large phase shift of up to $0.4 \mathrm{rad}$.

There are features in the reconstructions visible within sizes of one to two pixels indicating a half period length resolution in the range of the pixel dimension of $d x=46 \mathrm{~nm}$. The PRTF reflects a good reconstruction up to the highest frequencies. In comparison to the PRTF of the Siemens star (cf. Fig. 3.34(a)), one observes a lower trend in the high frequency region of the reconstruction of Fig. $3.42(\mathrm{~b})$. The PSD (blue curve, Fig. 3.34(b)) of the reconstruction in Fig. 3.43(b) clearly shows structures up to $d x=50 \mathrm{~nm}$ (half period length). The cut-off is very small and can be better seen in the 2D PSD of Fig. 3.34(d) indicating a resolution in the range of $d x=50-55 \mathrm{~nm}$.

The tomographic reconstruction (Fig. $3.43(\mathrm{~d})$ ) reveals the 3D structure of bacterial cells seen in Fig. 3.43(b), A video has been published on the internet, which shows a $3 \mathrm{D}$ visualisation of the cells ${ }^{20}$. One can identify the $3 \mathrm{D}$ distribution of the high-density regions (red). Assuming the ratio of the mass number and the atomic number on average over a voxel to be nearly constant, the 3D phase distribution $\phi(\vec{r})$ can be used to estimate the mass density of the cells ${ }^{21}[97$

$$
\rho_{c}(\vec{r}) \approx-\frac{2 u}{\lambda r_{0}} \phi(\vec{r}),
$$

where $u$ denotes atomic mass unit, $r_{0}$ the classical electron radius and $\lambda$ the wavelength. For cell material of $\mathrm{H}_{50} \mathrm{C}_{30} \mathrm{~N}_{9} \mathrm{O}_{10} \mathrm{~S}_{1}$ the error is less than $10 \%, 97$. The tomographic reconstruction is used to estimate the mass density of the highdensity regions. With the help of software tools (Avizo Fire 7.0.1, Visualization Science group, USA) for analysing tomographic data, a sub-volume was defined which can be seen as a magenta label in the lower part of the tomogram (frontview, Fig. 3.43(d)). The average over the magenta-coloured sub-volume yields $<\rho_{c}>\approx 1.6 \mathrm{~g} / \mathrm{cm}^{3}$. The $2 \mathrm{~d}$ projection of Fig. $3.42(\mathrm{a})$ was also used to obtain absolute mass values for the high density regions by integrating over their area. The results are shown in the histogram of Fig. 3.44(a), where the absolute values were normalized to the mean of $0.11 \mathrm{pg} \pm 0.04 \mathrm{pg}$ of the first group of bins. Notably, an approximate grouping around integer values is visible. Possible implications of this finding are discussed in section 3.1.12. Using the model of a sphere with diameter $2 r=500 \mathrm{~nm}$, one also obtains a total mass of $0.1 \mathrm{pg}$ when using the mass density of $1.6 \mathrm{~g} / \mathrm{cm}^{3}$. The integral mass of the cell cluster is $43.75 \mathrm{pg}$. Assuming the total mass belongs to 16 single cells, one obtains a value of $2.73 \mathrm{pg}$ of dry weight per single cell.

\footnotetext{
${ }^{20}$ The video in MPG format can be downloaded from http://www.opticsinfobase.org/oe/ viewmedia. cfm?uri=oe-20-17-19232\&seq=2

${ }^{21}$ See also equation $\overline{1.59}$, p. 23
} 
In conclusion, the results show that ptychographic tomography is a suitable approach not only for strongly scattering samples (e.g. mineralized tissue $[61]$ and stained bacteria [109]) but also for weakly scattering specimens such as freeze-dried cells. Note that ptychography from unmineralized tissues and in particular cells is limited by the signal to noise ratio. Thus weakly scattering samples are much more difficult to reconstruct than for example mineralized tissues such as bone or more generally solid state samples.

In comparison to the reconstruction of the single projection in Fig. 3.43 (b) which has been taken before the tomographic dataset, the quality of the tomographic reconstruction is affected by the overall consistence of all ptychographic reconstructions. The inner structure slightly deviates from the first phase map indicating a moderate radiation induced change of the inner structure. The applied dose of the tomographic dataset was estimated to be $\sim 2.4 \cdot 10^{8}$ Gy. Another tomographic dataset has been taken after the first one in order to achieve a smaller angular increment of $\Delta \Phi=1^{\circ}$. However, the combined tomogram (not shown) shows less contrast in the high-density regions and was thus discarded. The image in Fig. 3.43(c) has been recorded after an integral dose of $>5 \cdot 10^{8}$ Gy and clearly shows radiation induced structure changes in comparison to its initial state in Fig. 3.43 (b) Note, that the structural changes may also have an effect on $\left\langle\rho_{c}\right\rangle$. As the mass density obviously decreases, $\left\langle\rho_{c}\right\rangle$ can thus only be considered as a lower bound. The dose estimations are summarized in Table 3.8 (see also Fig. 3.38). It is thus concluded that in contrast to single projections, medium and high resolution tomography will necessitate cryogenic sample conditions.

Table 3.8: Overview of PCDI datasets according to dwell time $\Delta T$, grid spacing between adjacent rings $\Delta_{r}$ of a scan of one projection, fluence $F$ of a single projection and dose estimations $D$ of the whole reconstruction.

\begin{tabular}{lcccc} 
reconstruction & $\Delta T[\mathrm{~s}]$ & $\Delta_{r}[\mu \mathrm{m}]$ & $F\left[\right.$ photons $\left./ \mu \mathrm{m}^{2}\right]$ & $D[\mathrm{~Gy}]$ \\
\hline Fig. 3.41 & 0.2 & 0.5 & $3.1 \cdot 10^{8}$ & $6.7 \cdot 10^{5}$ \\
Fig. 3.43(a) & 0.2 & 0.28 & $2.3 \cdot 10^{9}$ & $4.9 \cdot 10^{6}$ \\
Fig. 3.43(b) & 0.4 & 0.28 & $4.7 \cdot 10^{9}$ & $9.9 \cdot 10^{6}$ \\
Fig. 3.43(c) & 0.4 & 0.25 & $5.9 \cdot 10^{9}$ & $1.3 \cdot 10^{7}$ \\
Fig. 3.43(d) & 0.2 & 0.35 & $1.5 \cdot 10^{9}$ & $2.4 \cdot 10^{8}$ \\
\hline
\end{tabular}




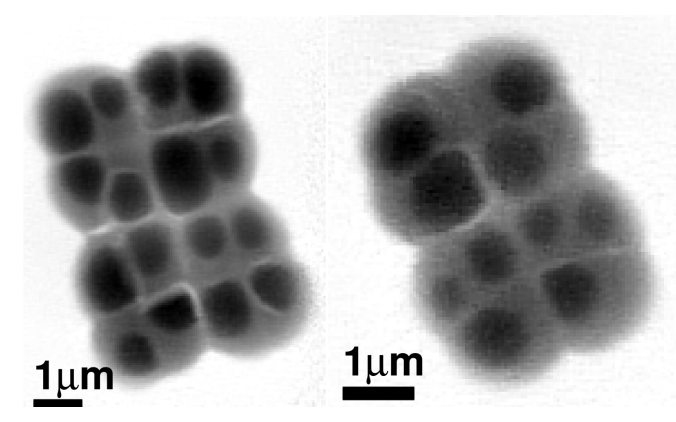

(a)

(d)

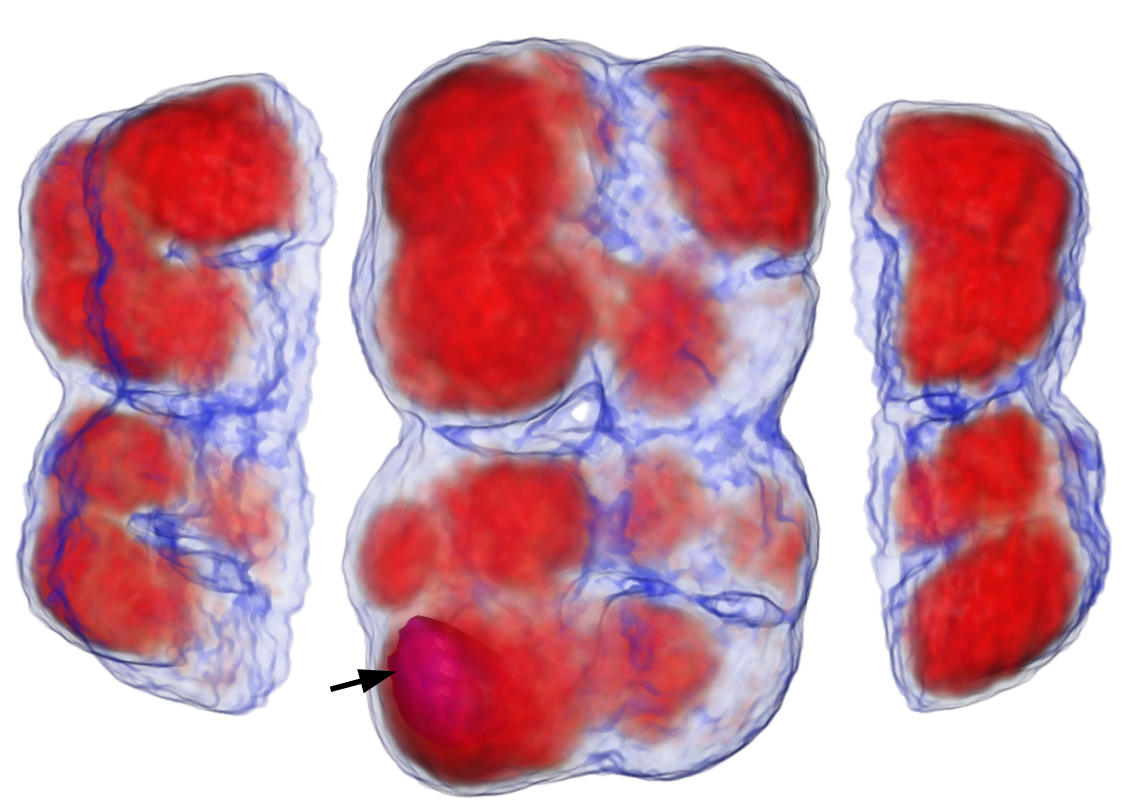

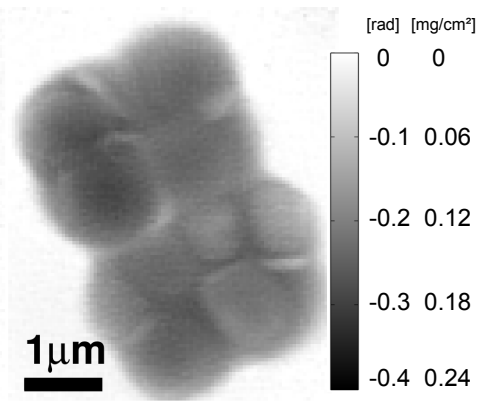

(c)

Figure 3.43: (a) -(c) Phase reconstructions of D. radiodurans ('DM' - algorithm) showing electron dense (dark) regions. (d) 3D visualisation of the tomographic reconstruction of the set of projection images of the cells in (b) (for a video cf. footnote on page 100 ). The tomographic reconstruction is shown from three different angles. A magenta label in the tomogram (black arrow, front-view) depicts voxel-regions which have been used to estimate the mass density $\rho_{c}$ in the high-density regions of the bacterial specimen (red). $\left\langle\rho_{c}\right\rangle \approx 1.6 \mathrm{~g} / \mathrm{cm}^{3}$ is obtained. (c) shows the cells of the tomogram (b) (d) shows the cells after taking an additional tomographic dataset revealing mass diffusion due to radiation damage. (a) (b) same colourbar as in (c). 


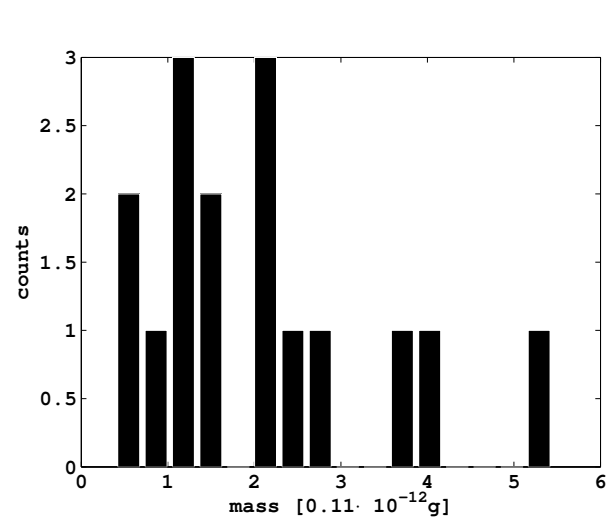

(a)

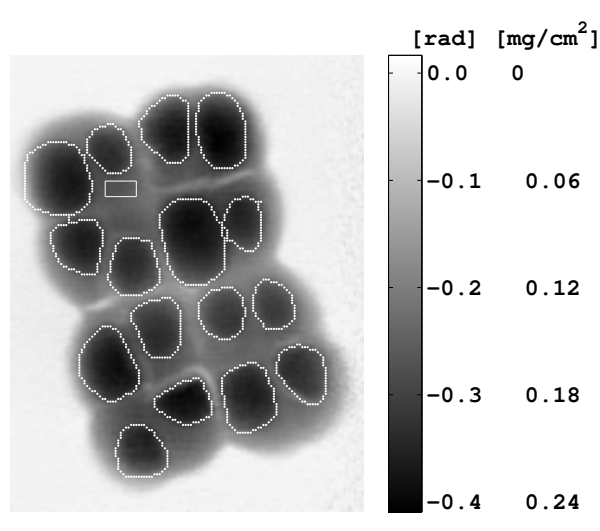

(b)

Figure 3.44: (a) shows a histogram of the mass values that were obtained by integrating (electron) dense regions in (b). The masses are normalized to a value of $0.11 \mathrm{pg}$ which corresponds to the mean value of the first group of bins in (a). The regions used for integration are indicated in (b) by white dots. Values are obtained after background subtraction according to the mean value of $0.159 \mathrm{mg} / \mathrm{cm}^{2}$ of the area within the white rectangle. Thereby, it is implicitly assumed that the dense regions are surrounded by a layer of material that contributes $0.159 \mathrm{mg} / \mathrm{cm}^{2}$ to the full projection.

\subsubsection{Results: TEM Analysis of D. radiodurans}

In order to better understand the cellular interior of D. radiodurans, ultra-thin sections of resin-embedded, chemically fixed and stained cells were prepared and analysed with Transmission Electron Microscopy (JEM-1011 Transmission Electron Microscope; Jeol, Japan). For this reason, cells were cultured similarly (incubation for about $36 \mathrm{~h}$ at $32^{\circ}$ ) as described above (see also section 1.3.10, p. 47). Note that the preparation for TEM images neither includes plunge-freezing nor freeze-drying (cf. section 2.3.3, p.59). The contrast of cellular features in TEM images crucially depends on the used fixation and staining methods. For this reason, the sample preparation was performed once with the use of osmium tetroxide $\mathrm{OsO}_{4}$ and once without, Fig. 3.46 and Fig. 3.45 respectively.

First, the images without osmium tetroxide treatement are discussed. The cells of $D$. radiodurans can be clearly identified in figure 3.45 . The cell morphology was not found to be homogeneous. For instance, the typical tetrads can be observed (Figs. 3.45(b), (c)) but more complex cellular structures, where e.g. a beginning cell division can be seen (Fig. 3.45(a)), are found as well. Many cellular details can be well resolved as already discussed in section 1.3.10. For instance, the cell wall including the holey-layer and possibly the cytoplasmic-membrane can be identified. In addition, ribosomes are widely distributed throughout the cell interior and the bacterial nucleoid can be identified, too. The DNA appears as ovoid discs of different diameters. It appears slightly brighter than the surrounding 
proteinaceous material. The maximum diameter seems to be in the range of about $600 \mathrm{~nm}$. The toroidal form can be seen, too (Fig. 3.45(d)). Other more undefined structures are also present.

Next, the preparation including osmium tetroxide will be addressed. The main difference in contrast in comparison to the images before arises in the cell wall and the nucleoid. In particular, the peptidoglycan containing holey-layer appears with strong contrast. The regular fine structure can be well resolved, e.g. Fig. 3.46(b) In addition, the contrast between proteinaceous material and the region containing DNA is higher. There are plenty regions appearing empty or white in the regions containing mostly DNA. Similarly, there are tetrads but also other types of cell formation. The image in figure $3.46(\mathrm{c})$ nicely illustrates the toroidal shape of the DNA in singular sections but other forms are also possible. For instance, in the upper, left cells of Fig. 3.46(d) the nucleoid can be seen before cell division (see also Fig. F.1, p. 196). One feature of the cell interior, that was also found in some sections of cells, are electron dense polyphosphate granules. One can be observed in Fig. 3.46(d), Its diameter is less than $500 \mathrm{~nm}$.

In conclusion, the state of the bacteria grown on agar plates under these conditions appears well preserved but one cannot deduce for example that all the cells are in the stationary phase. The state of these cells is rather diverse in this sense. 


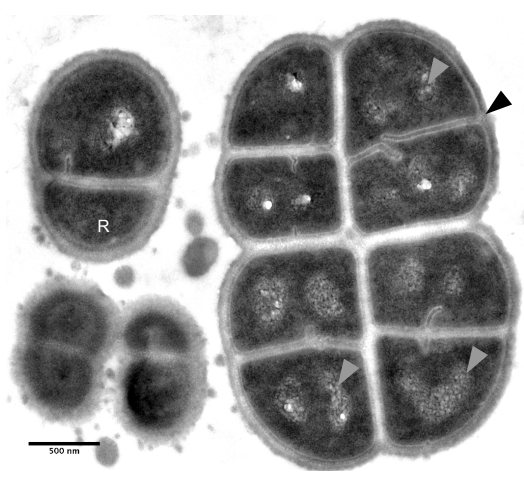

(a)

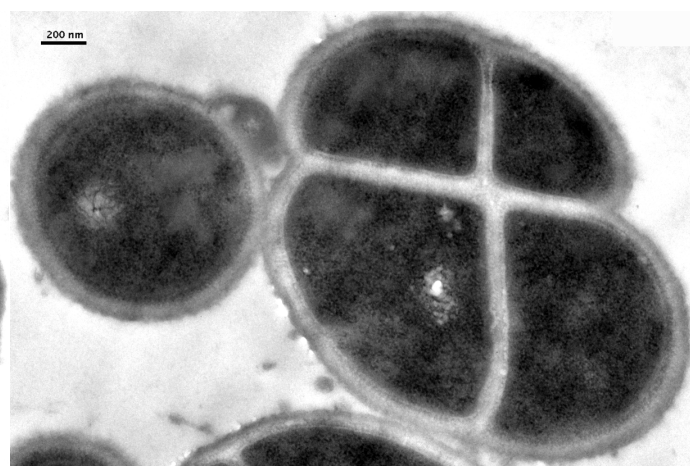

(b)

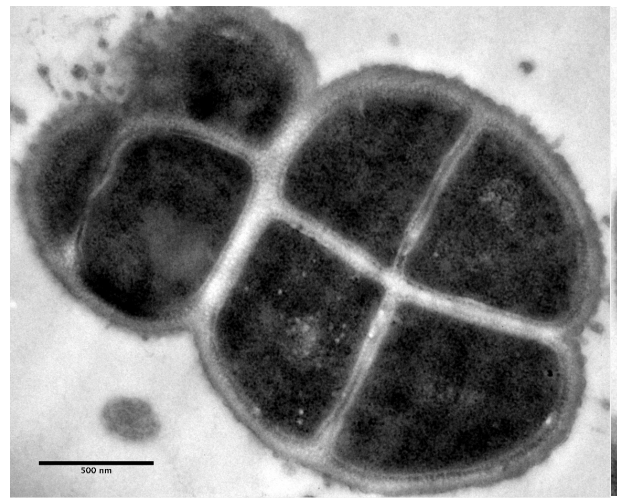

(c)

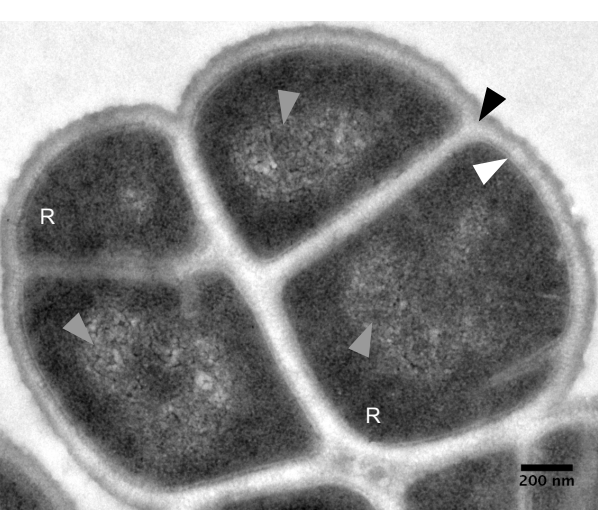

(d)

Figure 3.45: The figure presents TEM images of ultra-thin sections of $D$. radiodurans cells that have been chemically fixed, embedded and post-stained with uranyl acetate. In (a) an early stage of cell division can be seen. (b) and (c) show tetrads. Cell components such as ribosomes (indicated by the letter 'R'), DNA (grey arrowheads), the cell wall (black arrowheads) and possibly the cytoplasmic membrane (white arrowhead) can be identified. 


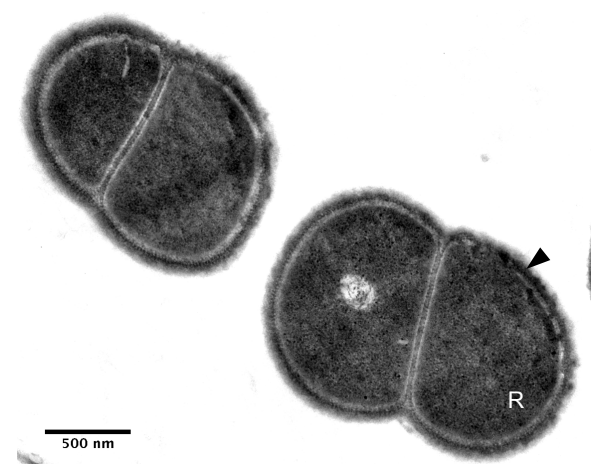

(a)

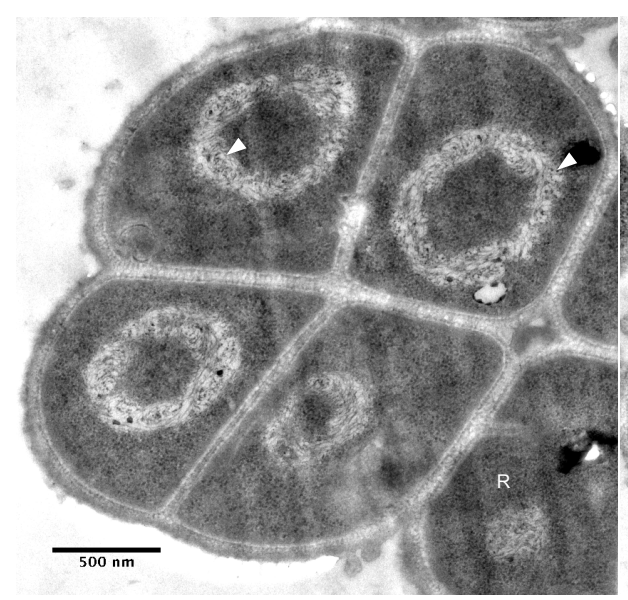

(c)

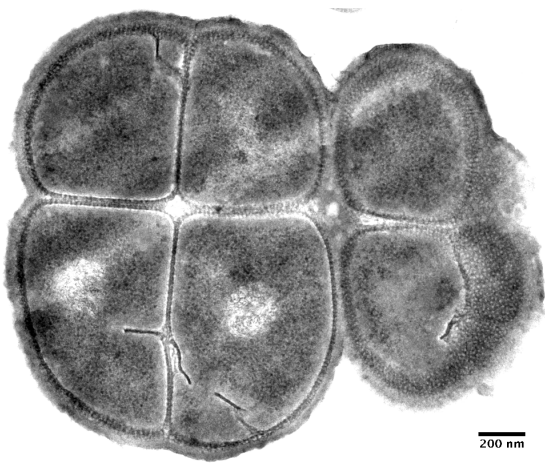

(b)

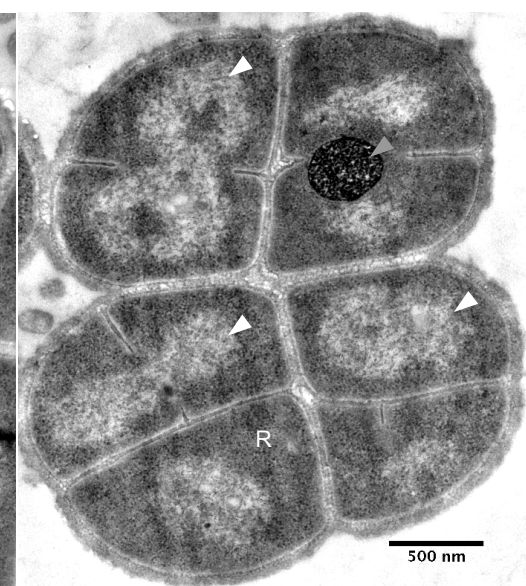

(d)

Figure 3.46: The figure presents TEM images of ultra-thin section of $D$. radiodurans cells that have been chemically fixed and stained (and fixed) with $\mathrm{OsO}_{4}$ prior to embedding and post-staining with uranyl acetate. (a) could be diplococci but more likely cross-sections through the upper or lower cells of a tetrad. In (b) the peptidoglycan containing holey layer within the cell wall can be seen. The DNA of the cells in (c) exhibits toroidal shape. The nucleoid in the cells in (d) occurs in different forms. Cell components such as ribosomes (indicated by the letter 'R'), DNA (white arrowhead), the cell wall (black arrowheads) and a polyphosphate granule (grey arrowhead) can be identified. 


\subsubsection{Discussion \& Conclusion}

In summary, an X-ray structural analysis on bacterial cells of D. radiodurans was carried out, combining coherent imaging and cellular nano-diffraction. For this study, the bacterial cells were vitrified by plunge freezing followed by freeze-drying. However, in order to minimize radiation damage, samples were at least for experiments with highest flux (incoherent setting) kept at cryogenic temperatures by the use of a cryogenic $N_{2}$ jet. This shows that the experimental scheme is compatible with future extension to frozen hydrated samples with near optimum structure preservation.

Primarily, it is shown how the cellular diffraction signal can be extracted and analysed which can be seen as a pioneering basis for follow-up works on cellular nano-diffraction such as $|323|$. Furthermore, the cellular diffraction curves were compared as a function of momentum transfer after azimuthal averaging, for different parameters of the illuminating beam, notably flux and coherence. The observed curves could all be described by a power law decay with an exponent $\nu$ in the range $-4 \leq \nu \leq-3$. Importantly, the cellular diffraction experiments open the analysis to maximum scattering vectors, up to a momentum transfer of $q_{\dagger} \approx 2 \mathrm{~nm}^{-1}$, which is to date unachievable by CDI. No indications for ordered structures associated with DNA compactification were observed in this range. Note, however, that the absence of peaks or modulations in the diffraction curves does not preclude ordered structures with signals below the dominating power-law decay.

In addition, it is shown how the ptychographically reconstructed probe can be directly used for enhanced control of the nano-diffraction experiment. Most importantly, the illuminated region of the sample can be well characterised. In particular, the size and shape of the beam does not have to be measured by knife-edge scans with the associated inaccuracies. Moreover, the exact complex-valued wave front is accessible, from which all beam parameters, that are desired to better control the nano-diffraction experiment, can be derived, such as wavefront curvature for example. Furthermore, the scanning X-ray microscopy images of differential phase contrast and darkfield contrast can be analysed with the full probe information at hand. Finally, it is also shown how dose estimations become more precise by taking into account the exact beam profile, as well as the overlap between serial exposures during scanning.

Next, the ptychographic results are summarized. The reconstruction yielded the projected phase (2D image) at a resolution in the range of $10 \mathrm{~nm}$ for the tantalum test pattern. In the future, reducing the distance between focus and sample, as well as switching to Fresnel zone plates with higher resolution will maximize the fluence on the sample and improve the sampling in the detection plane. The resolution of the phase maps of unstained, unsliced and freeze-dried cells of $D$. radiodurans is in the range of $50 \mathrm{~nm}$. Comparing the two ptychographic imaging experiments on the cells, a somewhat surprising result is obtained. Obviously, the fluence has been increased by about one order of magnitude but against the expectation an improvement in resolution is not clearly visible. In fact, the resolution may even be slightly lower. One reason seems to be a spatially slightly more incoherent beam 
in the second experiment due to a not optimum setting of the slit S0 as discussed in section 3.1.8. In addition, a proof-of-principle of tomographic ptychography of unstained and unsliced biological cells was presented. Imposing an additional phase constraint into the reconstruction scheme was found to be helpful as it removes unphysical phase offsets between different projections. For the case of isolated specimens, this facilitates a consistent tomographic reconstruction. No additional phase unwrapping was necessary, due to the low phase shift of unstained cellular specimens. However, the second tomogram (Fig. 3.43) shows that globular dense regions of ca. $1.6 \mathrm{~g} / \mathrm{cm}^{3}$ exist in the bacteria with approx. isotropic shape, which may be linked to the bacterial nucleoid and DNA. The TEM images of ultra-thin sections of chemically fixed, stained and resin embedded D. radiodurans cells give a good overview of the cellular fine-structure. However, an interpretation with respect to the projected or $3 \mathrm{~d}$ phase reconstruction is not straight forward as only single slices of different cells were obtained. Nevertheless, the DNA can be observed in different arrangements. Considering the size of DNA containing regions within these sections, one notices that it is often well below a diameter of $1 \mu \mathrm{m}$. This is smaller than the size of the dense regions that are visible in the ptychographic reconstructions, where this pronounced feature observed in density contrast appears to be sometimes larger than $1 \mu \mathrm{m}$ in the projection. On the other hand, the only other feature that was observed in the TEM sections are electron dense polyphosphate granules, which appear to be much smaller in size. A striking characteristic of the dark phase globules is that they seem to appear once per cell as one would expect from the nucleoid. The broad size distribution between different cells may be due to the diverse state of the cells, i.e. there may be multiple copies of the genome present within the cells during cell division. A model based determination of the total dry weight of the dense regions from a $2 \mathrm{~d}$ projection indicates that their masses may be approximately grouped in integer multiples of $0.11 \mathrm{pg}$, which supports the hypothesis of multiple genome copies being present in a single cell. The electron dense regions of the ptychographic phase reconstructions may thus be tentatively linked to the bacterial nucleoid and DNA but more data is needed to further support this hypothesis. In the future, improvements towards a more defined state of the cell culture in combination with a stack of TEM images of the same cells may be helpful. A combination with visible light fluorescence microscopy seems also appealing. In case of freeze-dried samples, DNA staining with DAPI was performed but the fluorescence appeared to be not preserved after freeze-drying, thus hydrated specimens may be necessary. An increase in resolution of the ptychographic phase maps will also facilitate further interpretation. In contrast to the $2 \mathrm{D}$ case, the resolution of the ptychographic tomogram was limited due to radiation induced damage in both experiments. Here it is stressed that the radiation damage in the first experimental approach is more severe. The second tomo-ptychographic dataset does not suffer from a degradation on the same level. Instead, only a slow effect of mass diffusion can be observed over the timescale of about 70 to 140 projections. In any case, the next step must thus be ptychographic imaging of frozen-hydrated samples both in $2 \mathrm{D}$ and $3 \mathrm{D}$ as was exemplified by Lima and colleagues $[172]$ in $2 \mathrm{~d}$. 
However, one must be aware of the limitations in the presented approach of a combination of information from both real and reciprocal space. While the resolution can be extended by analysis in reciprocal space, this resolution applies to the averaged structure. The real space resolution of this averaging process as given by the focal spot is clearly lower in cellular nano-diffraction than in ptychographic imaging or more generally in CDI. At the same time, the overhead for detector read-out during scanning of three degrees of freedom needed for tomography limits the reasonable field of view, and hence in most cases the size of the object. The combination of high resolution imaging techniques such as ptychography with cellular nano-diffraction techniques, that give access to an even higher resolution in reciprocal space, is of utmost importance. 


\subsection{Scanning Hard X-ray Microscopy Imaging Modalities for Geobiological Samples}

Modern Scanning X-ray Microscopy can help to unravel the spatial context between biotic and abiotic compounds of geobiological assemblies with the aim to finally link chemical pathways to biological activities at the nanometre scale. The small-scale visualisation of the spatial context between prokaryotic and/or eukaryotic organisms, extra- and intracellular compounds and minerals is crucial for the attempt to ultimately unravel a large-scale functioning of geomicrobiological consortia. To date, X-ray microscopes offer a variety of probing techniques that, for instance, have applications in the material, environmental and life sciences making use of either full-field or scanning set-ups that are illuminated by synchrotron, compact laboratory or X-ray free electron laser sources (for reviews see [161,256]). Small wavelengths, thus large penetration depth and a high spatial resolution combined with elemental speciation and potentially information about the chemical state turn X-rays also into an ideal tool for addressing geobiological questions via qualitative and quantitative imaging applications. Due to the manifold of possible X-ray microscopy techniques, a careful combination of different imaging modalities is of utmost importance and exemplifying work is needed when new techniques arise. Some applications of soft X-ray microscopy modalities can be found in the works of $100,188,271,300,301]$ and the reviews $[50,161,167,219,256,290$ and references therein. Recent applications with a geobiological context are also given in the works of $22,131,204,218$ to name a few examples.

Here the presented experiments use the multi-modal imaging techniques provided by hard X-ray microscopes at synchrotron radiation sources to address analytical needs in geobiological research. Using the examples of 1) micron sized $\mathrm{CaCO}_{3}$ spherulites as basal elements of the aragonitic skeleton of the coralline demosponge Astrosclera willeyana (e.g. [145, 173,338]) and 2) a subsurface consortium of filamentous sulfur-oxidizing bacteria (SOX), the potential of Scanning X-ray Fluorescence and Scanning Transmission X-ray Microscopy (STXM, 'STXM' includes darkfield and differential phase contrast) and the quantitative approach of Ptychographic Coherent Diffractive Imaging (PCDI) at single cell level is illustrated. In particular, the latter sample was scanned for extra- and intracellular sulfur compounds (e.g. [185, 276]) and subjected to the modern X-ray phase contrast method ptychography as a kind of feasibility study with the aim to obtain quantitative phase contrast (mass density) images of single cells.

This section is partly based on a manuscript that has been accepted for publication 331 . The Astrosclera willeyana data has been recorded in the context of another work. However, the data was not fully analysed and interpreted in 328 . The new results of the full analysis are thus presented in the context of this work. Complementary to the presentation in |331|, more details about the ptychographic reconstruction of the SOX sample are included in section 3.2.3. In addition, a SEM-EDX spectroscopy has been carried out on the same sample after the ptychographic imaging experiment in order to search for bacterial sulfur inclusions. The preparation of the SOX sample by Nadia Quéric is gratefully acknowledged. 


\subsubsection{Sample Origin \& Sample Preparations}

Primary aragonitic skeletal elements were prepared from the outermost zone of the coralline demosponge $A$. willeyana (e.g. [145, 173, 338]). Sponge tissue, including the proximal ectosome, was dissolved in sodium hypochlorite until only parts of the calcified skeleton remained. After air drying, the larger fragments were removed through successive sieving using mesh sizes of $125 \mu \mathrm{m}$ and $25 \mu \mathrm{m}$ (RETSCH). The obtained powder containing many isolated spherulites was then scattered on a $200 \mathrm{~nm}$ thick $\mathrm{Si}_{3} \mathrm{~N}_{4}$ foil (SILSON, TEM standard) and fixed with nitrocellulose lacquer (see also 328 ).

Bacterial aggregations dominated by sulfur-oxidizing communities were collected in May 2009 during the monitoring sampling campaign within the project 'Microbial biomineralization, biogeochemistry and biodiversity of chemolithotrophic microorganisms in the tunnel of Äspö (Sweden)' (FOR571, Deutsche Forschungsgemeinschaft), at the Äspö Hard Rock Laboratory. A feather-shaped sample of predominantly filamentous bacteria of more than $100 \mu \mathrm{m}$ length was collected at station 1310B (cf. Quéric et al. [242]). The sample was chemically fixed by glutardialdehyde ( $4 \%$ in $1 \mathrm{x}$ PBS) and stored at $4{ }^{\circ} \mathrm{C}$ until further processing. In the next step, a subsample was first washed with $1 \mathrm{x}$ PBS and then thoroughly spread out onto a $1.5 \mathrm{~mm} \times 1.5 \mathrm{~mm}, 500 \mathrm{~nm}$ thick $\mathrm{Si}_{3} \mathrm{~N}_{4}$ membrane (SILSON). The sample dehydration by rising ethanol concentrations of $15 \%, 30 \%, 50 \%$ and $70 \%$ was carried out directly on the membrane for 30 min each, followed by $90 \%$ and $99 \%$ for $60 \mathrm{~min}$ both. A last dehydration step was carried out chemically via HMDS (hexamethyldisilazane).

\subsubsection{Details: Scanning X-Ray Fluorescence and Scanning Transmis- sion X-Ray Experiments}

The experiments on $A$. willeyana were carried out at the hard X-ray nanoprobe microscope at the ID13 beamline of the European Synchrotron Radiation Facility (ESRF) in Grenoble, France, using monochromatized (Si(111) channel-cut) undulator radiation of $15.25 \mathrm{keV}$ photon energy. The beam was focused in both the horizontal and vertical direction by a pair of nanofocusing refractive X-ray lenses (Schroer et al. 2005) located at $96 \mathrm{~m}$ behind the undulator source (cf. Fig. 3.47 , p. 112). The beam size was measured in the focal plane along horizontal and vertical profiles to be $78 \mathrm{~nm} \times 86 \mathrm{~nm}(\mathrm{FWHM} \mathrm{h} \times \mathrm{v})$ with the ptychographic method (Schropp et al. 2010). A platinum pinhole was placed behind the lenses to shield the sample from unfocused radiation. The samples were mounted on top of a high precision piezo stage (Physik Instrumente, Germany) a few millimetres behind the lenses. An energy dispersive detector (Vortex-EM, SII Nanotechnology USA) with an active area of $\sim 50 \mathrm{~mm}^{2}$ approached the sample from the side at an angle of approx. $45^{\circ}$ with respect to the optical axis. The distance between the sample and the fluorescence detector was around $1-2 \mathrm{~cm}$. The single photon counting detection device MAXIPIX |176, 234,235| was placed on the optical axis at a distance of $1.926 \mathrm{~m}$ from the focus. The active area of this detector covers $256 \times 256$ pixels with a size of $55 \mu \mathrm{m}$. The MAXIPIX records the (coherent) small 
angle X-ray scattering (diffraction) signal and can be used simultaneously with the fluorescence detector. The photon flux was measured with the MAXIPIX to be approx. $2 \times 10^{7}$ phot/s. A helium filled flight tube between sample and diffraction detector was used to minimise scattering of X-rays in air.

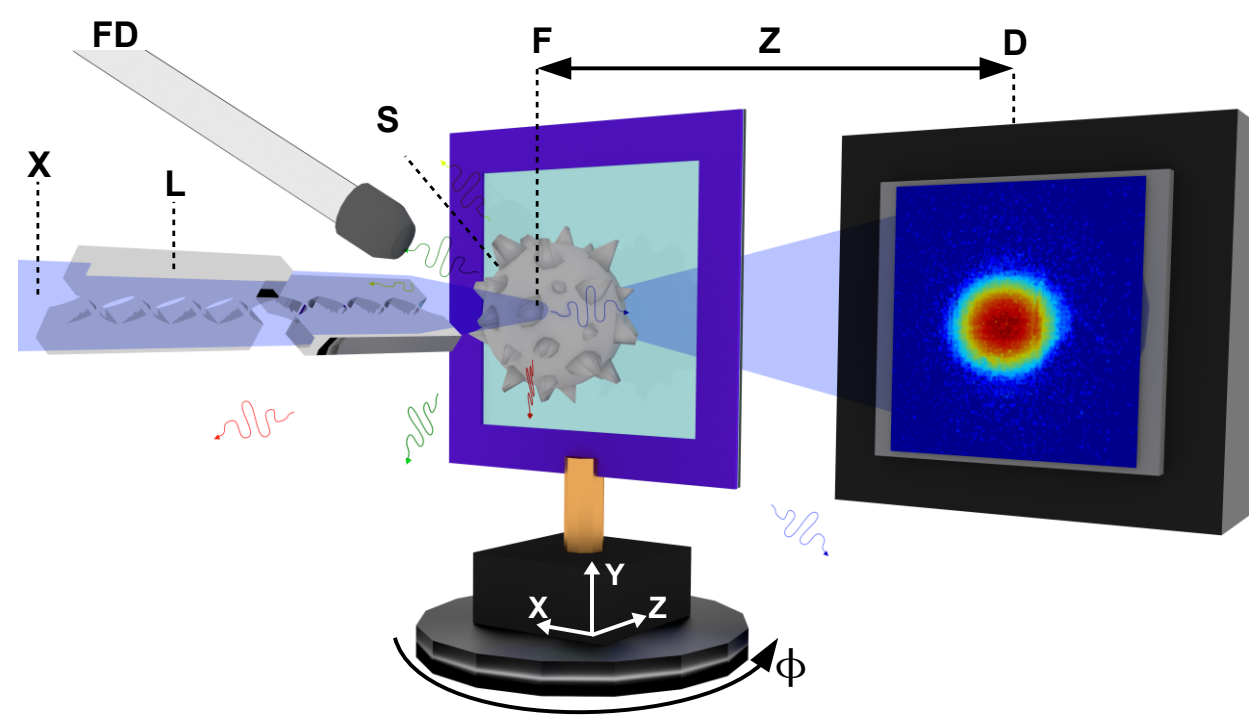

Figure 3.47: Schematic set-up of an X-ray Scanning Microscope: (X) incoming X-ray beam, (L) X-ray lenses, (F) X-ray focus, (S) sample on top of a rotation stage $(\Phi)$ for tomographic applications, (FD) X-ray fluorescence detector (close to the sample) and (D) pixelated diffraction detector located at a distance $Z$ of about several metres behind the focus. The flight tube being typically used between sample and diffraction detector is not shown.

The first scan over an entire spherulite of $A$. willeyana (S1) was recorded with $61 \times 61$ scan points on a rectilinear grid with step sizes of $500 \mathrm{~nm}$ in the horizontal and vertical direction. The exposure time was $0.1 \mathrm{~s}$ at each point. The beam size was adapted to the comparably large step size by measuring $0.5 \mathrm{~mm}$ out of focus. The follow-up scan (S2) covered a spike-shaped calcified extension at the outer part of the spherulite and was carried out $250 \mu \mathrm{m}$ close to the focal plane with a step size of $125 \mathrm{~nm}$ and $41 \times 41$ points. The exposure time was $0.1 \mathrm{~s}$ at each point. The scan S3 was taken on a subregion of S2. It consists of $31 \times 31$ scan points. The step size was set to $50 \mathrm{~nm}$ in both directions and the exposure time was $5 \mathrm{~s}$ at each point.

The fluorescence data were analysed using the PyMCA software 280. The summed spectrum of a region of interest (ROI) can be corrected to an otherwise defined background (BG) by subtracting at each scan point of the ROI an average spectrum of the BG. Here, BG-corrected spectra have been calculated for the scans S1-S3. Presented element specific fluorescence maps correspond to a summation of 
the fluorescence signal around $\pm 200 \mathrm{eV}$ of the $\mathrm{K}_{\alpha}$ emission line. STXM images, i.e. transmission, darkfield and differential phase contrast, have been calculated from the recorded diffracted intensities according to existing formulae [207,208,295] (cf. section 1.3.1, p. 34).

\subsubsection{Details: Scanning Transmission X-Ray and X-Ray Ptychography Experiment}

The imaging experiments on the sample of the consortium of filamentous SOX bacteria were performed at the coherent Small Angle X-ray Scattering (cSAXS) beamline of the Swiss Light Source (SLS) at the Paul Scherrer Institut in Villigen (Switzerland) under conditions similar to 330 as described in section 3.1 (p. 69). The sample was scanned by a high precision piezo stage (Physik Instrumente, Germany). The PILATUS 2M (SLS detector group, |163) single photon counting detector with zero-readout noise was used to record the diffraction data at a distance of $7.22 \mathrm{~m}$ behind the sample. A helium filled flight tube of length $7 \mathrm{~m}$ was installed between sample and detector for an optimized signal-to-noise ratio on the detector.

The STXM scan (Fig. 3.55 (b)-(e)) was taken on the sample using a rectangular mesh of $121 \times 121$ scan points with a step size of $0.5 \mu \mathrm{m}$. The sample was scanned in continuous mode along the vertical direction with an exposure time of $0.03 \mathrm{~s}$ allowing rapid data collection. The ptychographic images (Fig. 3.56) correspond to 7 single ptychographic scans taken along the sample that have been reconstructed unitedly. Each single scan covers an area of about $10 \mu \mathrm{m} \times 10 \mu \mathrm{m}$ and consists of more or less 323 scan points which are homogeneously distributed along concentric circles. Reconstructions of the single datasets are illustrated in Fig. 3.48. The exposure time was $0.2 \mathrm{~s}$ at each scan point. The photon flux on the sample was measured with PILATUS to be $\sim 4 \times 10^{8} \mathrm{phot} / \mathrm{s}$. The ptychographic reconstruction procedure of single scans using the same experimental set-up was already outlined in $\mid 330$ (cf. section 3.1, p. 69). Briefly, the reconstructions of the singular scans were initiated with a unit amplitude, constant phase object and a probe reconstruction from a Siemens star test pattern. The 'ePIE' algorithm [183 was run for 320 iterations using the algorithmic parameters $\alpha=\beta=0.5$. During the iteration the amplitude of the object was constantly clipped onto the interval $[0.7,1]$. The final results are the average that was taken over the last 200 iterations (every second iterate).

Here, the 'ePIE' ptychographic reconstruction algorithm was adapted to allow for reconstruction of multiple overlapping single scans. The idea to combine different ptychographic datasets was originally presented at a conference 60 . The first challenge, however, are positional errors that arise over the comparably long time being necessary for recording the data of all the scans. A reconstruction without refinement of the scan positions yields unsatisfactory results as can be seen in Fig. 3.49(a). Different algorithms and strategies have been combined to allow for positional refinement. Algorithms working on the level of single scan points $[20,182,307$ have not been considered due to the large overhead in compu- 


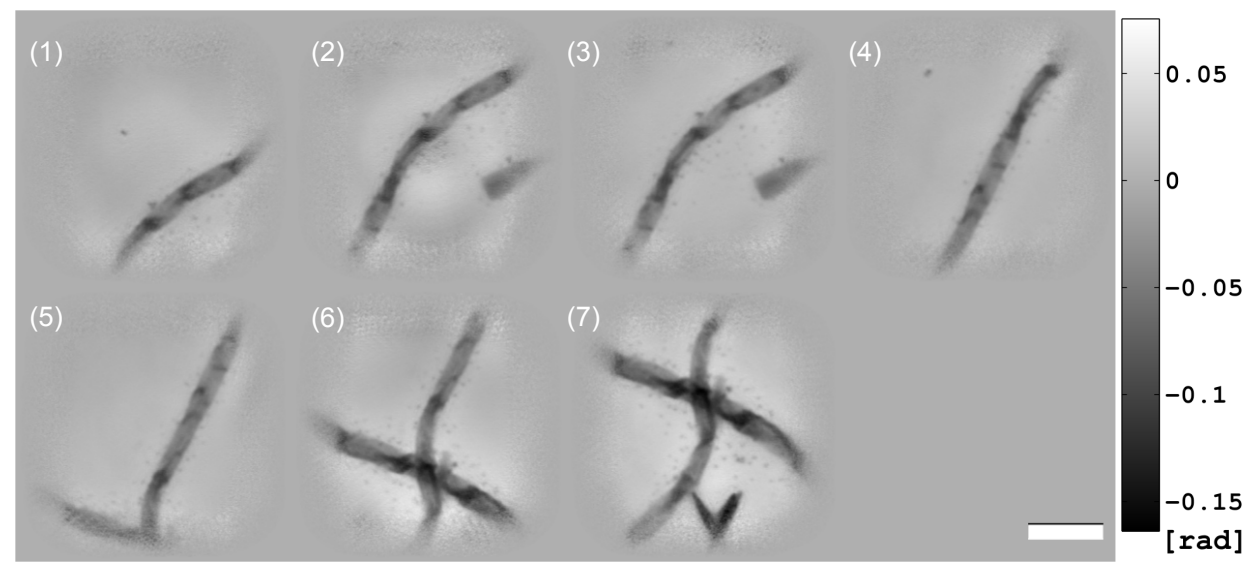

Figure 3.48: The image presents ptychographic phase reconstructions of single scans (1)-(7). Each sample area corresponds to an individual dataset and reconstructed with individual probe. The scale bar denotes $5 \mu \mathrm{m}$.

tation time. Instead, the good quality of the phase reconstructions of the single scans suggests a cross-correlation based method between the different reconstructions. For instance, the algorithm in $\left[113 \mid\right.$ is easy to implement ${ }^{22}$ but was found difficult to use on the data. Therefore, the MosaicJ plugin of the open-source ImageJ software project $[237,261,262,292]$ was used to create an image covering the full area of all the scans. Importantly, the grey values of the output image do not represent quantitative phase values anymore. Nevertheless, the image could be used as a target image for finding the positional errors with the help of the algorithm in 113 and the reconstructions of the single scans. The resulting corrections are presented in Fig. $3.49(\mathrm{c})$.

Simply using these corrections is not sufficient to obtain a high quality reconstruction as can be seen in figure 3.49 (b). The reason for unsatisfactory reconstruction results are due to a small change of the illuminating wave-field over the time of all the scans (about $35 \mathrm{~min}$ ). Hence, an improvement can be obtained by allowing for a reconstruction of multiple probes within the whole combined dataset. Here, the single probes correspond to the positions of the original single scans. That is, the final reconstruction yields one large object and 7 probes. The probes of the final object of figure 3.56 are presented in Fig. 3.50 in amplitude and phase. Here it should be noted that the change between the probes can hardly be deduced by eye-inspection. Comparing the amplitudes (Fig. 3.50(a)) reveals that the two upper maxima merge from (5) to (6). The changes were also quantified by calculating the difference between the complex probe field $P_{i}$ of scan $(i)$ and of scan $(i-1)$ and between scan $(i)$ and scan $(1)$. The same measure was calculated

\footnotetext{
${ }^{22}$ The source code can be obtained from http://www.mathworks.com/matlabcentral/ fileexchange/18401-efficient-subpixel-image-registration-by-cross-correlation (14/08/2014).
} 
seperately for amplitude and phase, too. The results are presented in figure 3.51 Calculations were obtained by using the following error metric

$$
\chi^{2}(A, B)=\frac{\sum|A-B|^{2}}{\sum|B|^{2}},
$$

where the summation is over all pixels of the fields $A, B$. The graph of figure $3.51 \mathrm{a}$ shows that there is a moderate increase of the error from (1) to (7) (squares) which mainly arises from differences in the phases (circles). The error remains about at the same level between neighbouring probes (cf. Fig. 3.51b).

The final reconstruction result has been obtained similarly as for the case of the single datasets. The 'ePIE' algorithm was run for 500 iterations on the full object region using the probes from the single reconstructions as initial guesses. The clipping of the object was carried out during each iteration, too. The average was taken over every second iterate during the last 200 iterations. Finally, the phase map of the full object region (Fig. 3.56) was converted into projected effective mass density values as outlined by Giewekemeyer and co-workers [97] (see also eqn. 3.121 , p. 100 and section 1.2 , p. 20). Here the following notation is used

$$
\sigma_{m}(x, y)=-\frac{2 u}{\lambda r_{0}} \phi(x, y),
$$

where $\phi(x, y)=\arg (O(x, y))$ is the two-dimensional phase of the reconstructed object function $O(x, y) . u=1.661 \cdot 10^{-24} \mathrm{~g}, r_{0}=2.82 \mathrm{fm}$ and $\lambda=0.2 \mathrm{~nm}$ denote the atomic mass unit, the classical electron radius and the wavelength. In addition, the projected attenuation is defined as

$$
\sigma_{\mu}(x, y)=-2 \log (|O(x, y)|)
$$




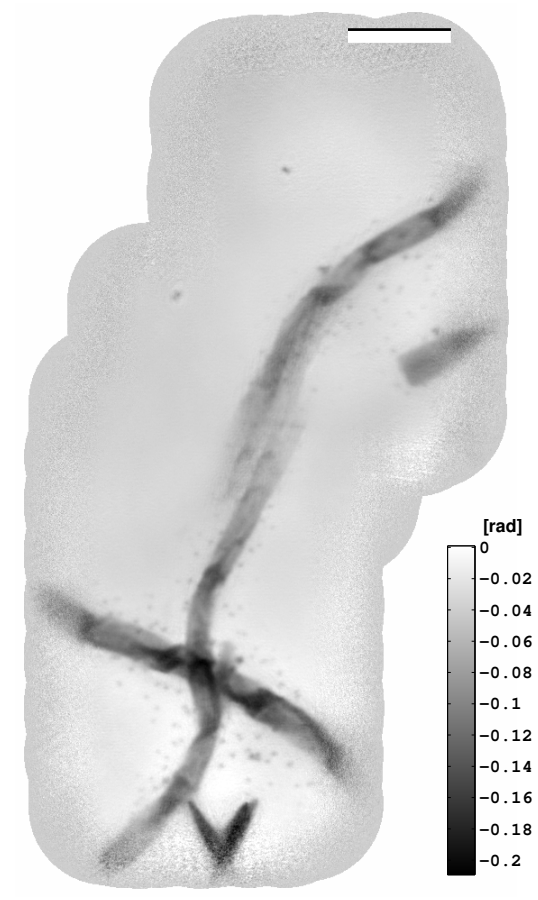

(a)

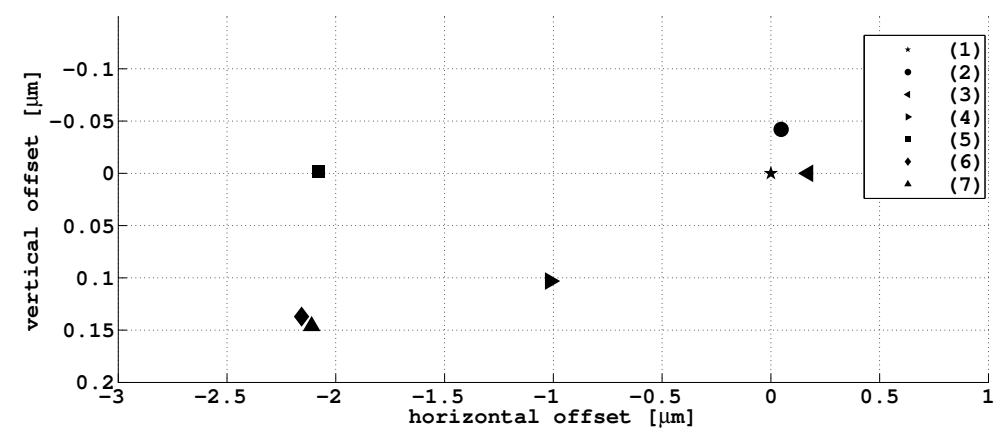

(c)

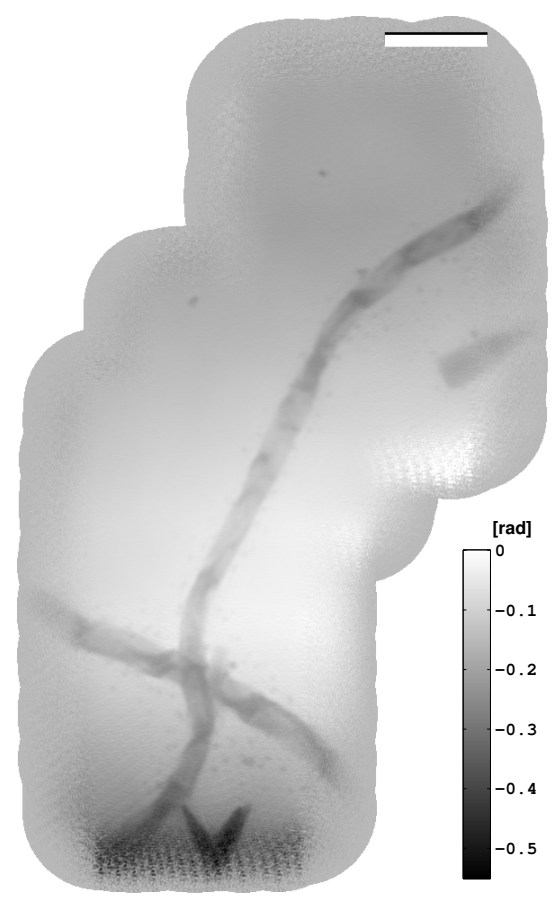

(b)

$$
\text { . }
$$

Figure 3.49: The figure illustrates two different problems that can spoil the combined reconstruction. (a) presents a combined reconstruction using the original positions and different probes of the subsets (1)-(7) (cf. Fig. 3.48). The positional discrepancy is clearly visible at the region of the subsets (3)-(5). (b) shows the combined reconstruction using refined positions but a single probe for all the subsets. A considerable phase background that changes along with the different scans can be seen. (c) shows the positions refinement for the scans with respect to measured motor positions, along the horizontal and vertical positions respectively. The scale bars in (a) (b) denote 5 $\mu \mathrm{m}$. 

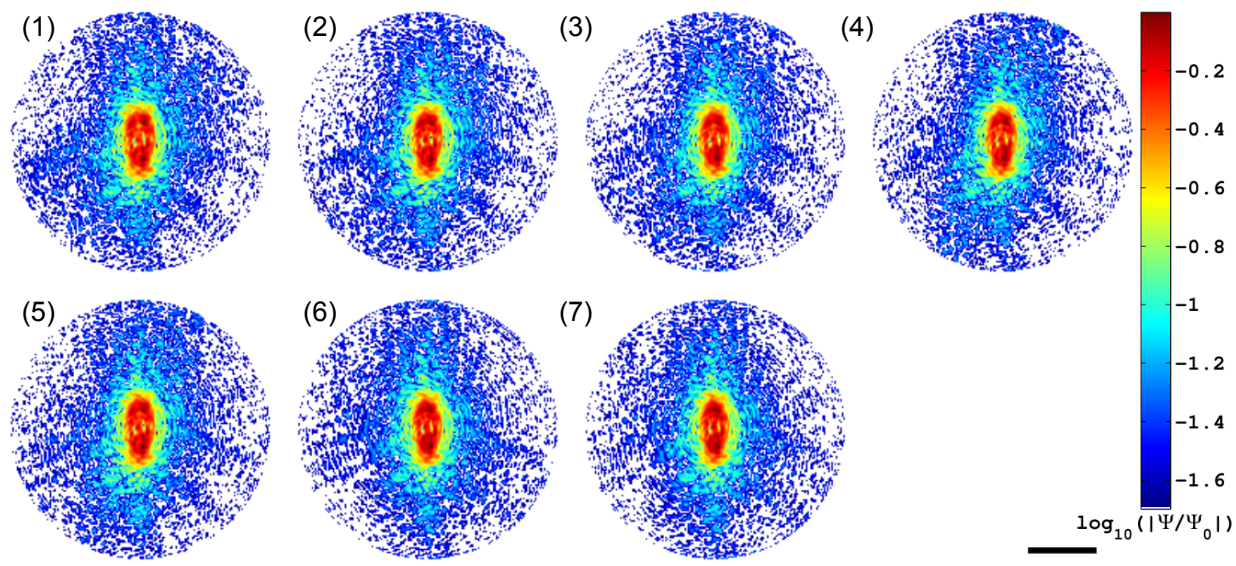

(a)
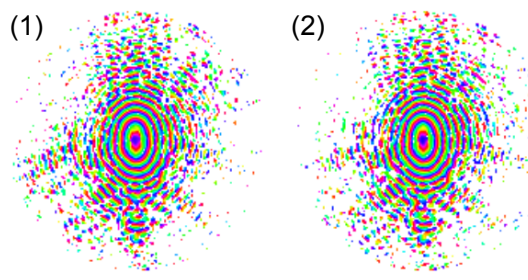

(3)

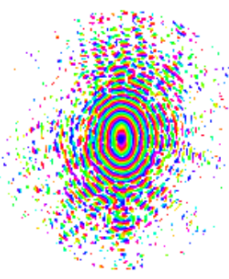

(5)

(6)

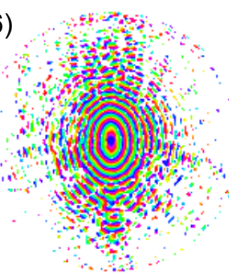

(7)
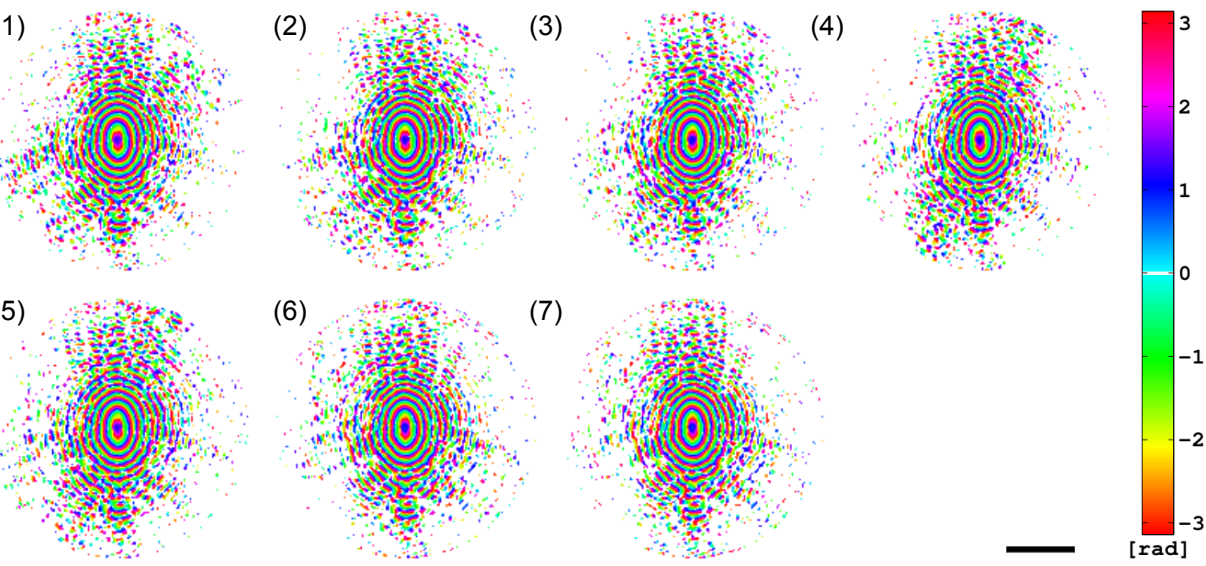

(b)

Figure 3.50: The figure presents probe reconstructions corresponding to the positions of the single scans: (a) amplitude and (b) phase. The amplitude is thresholded and the phases are only shown for amplitude values $>10^{-1.5}$. The scale bars in (a) (b) denote $2 \mu \mathrm{m}$. 


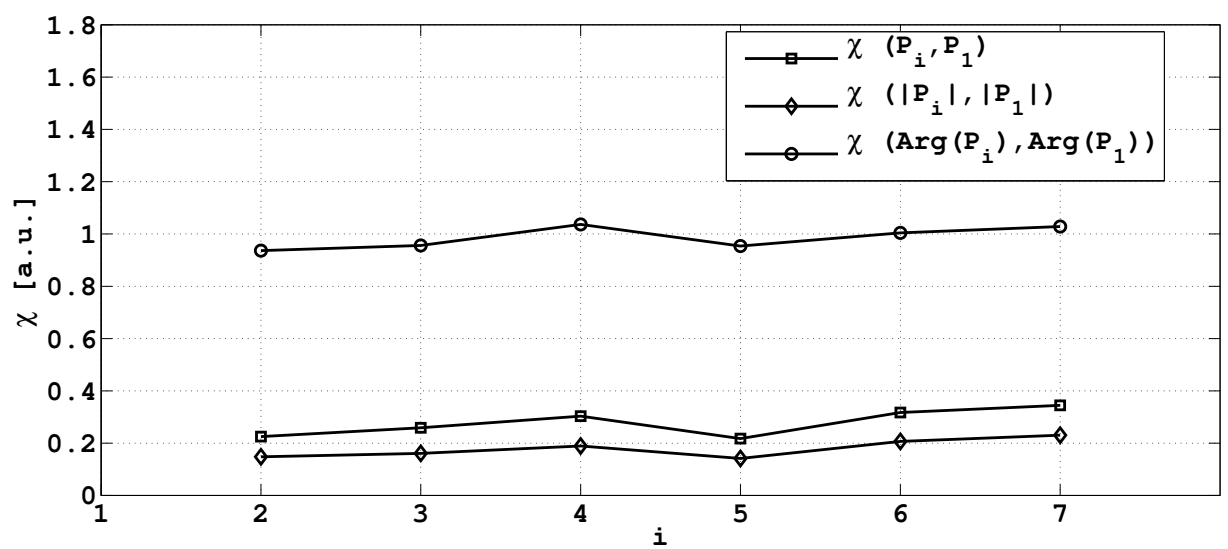

(a)

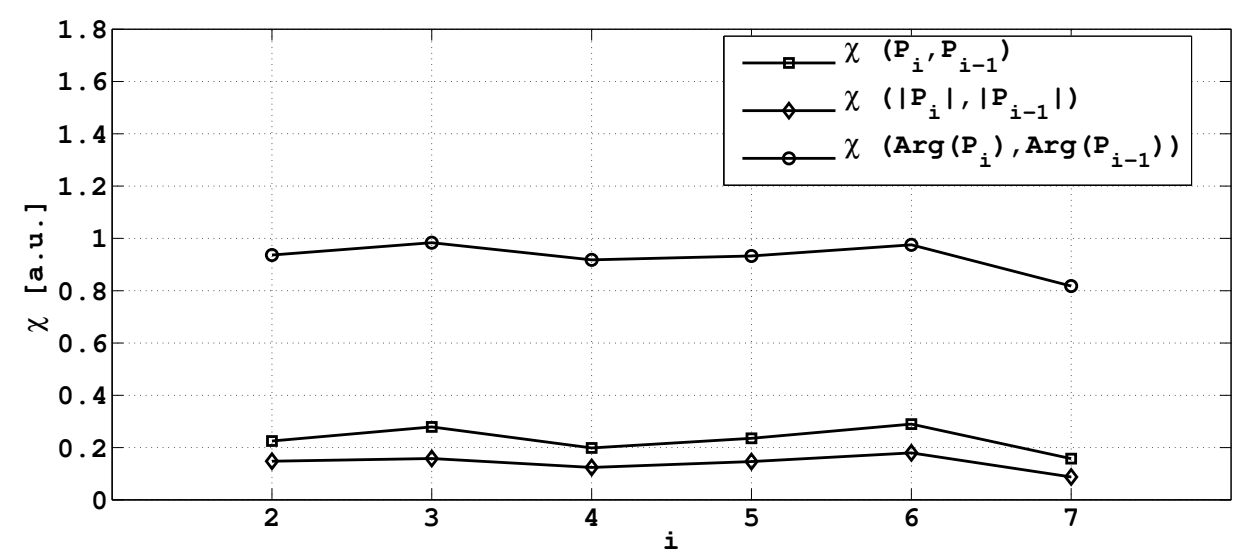

(b)

Figure 3.51: The figure shows the calculated error (a) between the probe $(i)$ and the probe (1) and (b) between the probe $i$ and the probe $(i-1)$ for the complex field, the amplitude and the phase. 


\subsubsection{Results}

The aragonitic spherulite of $A$. willeyana yields a dominating calcium signal in the summed fluorescence spectra of all scans S1-S3 (cf. Figs. 3.52 (a), 3.53 (a) and 3.54 (a)). The calcium map of S1 (Fig. 3.52 (c)) from the $\mathrm{Ca}\left(\mathrm{K}_{\alpha}\right) \mathrm{X}$-ray emission line at $3.69 \mathrm{keV}$ shows a clear projection of the spherulite. It can be seen that this sample consists of a central core of about $20 \mu \mathrm{m}$ diameter and tooth-formed extensions of $3-4 \mu \mathrm{m}$ length originating from the surface. Besides the calcium signal, small concentrations of chromium, manganese, iron, copper, zinc and bromine are well fitted to the spectrum. From these elements, the small contributions from the bromine peak at $11.92 \mathrm{keV}$ can still be visibly correlated to the sample (cf. Fig. 3.52 (d)). From the background-corrected spectrum (Fig. 3.52 (b)), one deduces that also the peaks from manganese, iron, copper and zinc can be attributed to the spherulite. However, the contribution is very low as can be seen in the fluorescence maps of manganese and zinc, Fig. 3.52 (e) and (f) respectively. Besides, it should be noted that the shown elemental maps correspond to the full signal, including the background. In case of the zinc data, the comparably small signal of the spherulite is difficult to distinguish from the background but occurrences of counts appear denser in the region of the spherulite.

In addition, the spherulite can be observed in the STXM images (Figs. 3.52 (g)(i)). The measured absorption is very low yielding insufficient imaging contrast (not shown). In contrast, the differential phase contrast in the horizontal (Fig. $3.52(\mathrm{~g})$ ) and vertical (Fig. $3.52(\mathrm{~h})$ ) directions yield a very good image of the sample. A composite image of the differential phase contrast and the fluorescence channels of $\mathrm{Br}$ (green), Mn (blue) and Zn (red) is presented in Fig. 3.52 (i). The composite allows for locating these elements on the spherulite.

The scan S2, over a single spike (Fig. 3.52 (g), arrow) with higher spatial sampling, is presented in figure 3.53 . The summed fluorescence spectrum (Fig. 3.53 (a)) is very similar to the one shown in figure 3.52 (a). The sample can be best visualised on the calcium map (Fig. 3.53 (c)) and the calcium peaks are clearly visible in the background-corrected spectrum (Fig. 3.53 (b)). In addition, there are weak contributions from elements such as zinc and bromine. For instance, the bromine map (Fig. 3.53 (d)) appears to be correlated to the spike shape while the zinc channel (Fig. 3.53 (e)) shows three peaks clearly located off the spike. In contrast to the scan S1, the differential phase contrast images of a single spike (Fig. 3.53 (f), (g)) show more surface details and a higher sensitivity to supposedly soft biological tissue that can be seen e.g. at the spike base. The composite consisting of the differential phase contrast and the fluorescence channels elucidates the distinct arrangement of $\mathrm{Br}$ (green), Ca (blue) and Zn (red) (Fig. 3.53 (h)).

Scan S3 that was carried out with highest spatial sampling and longest exposure times is presented in figure 3.54 (spike detail from Fig. 3.53 (f), rectangle with dashed white lines). Notably, a zinc peak can be clearly observed in the background-corrected summed spectrum (Fig. 3.54 (b)), originating from a single spot (cf. Fig. 3.54 (d)) on the completely calcified spike surface (Fig. 3.54 (c)). 
The STXM images of the sulfur-oxidizing communities (SOX) show a ROI of about $60 \mu \mathrm{m} \times 60 \mu \mathrm{m}$, which includes two intersecting filaments of SOX bacteria (Figs. 3.55, 3.56). The transmission map (Fig. 3.55 (b)) shows a high contrast between dense crystalline structures and cellular filaments. In contrast to thin cellular tissue, the crystalline material exhibits a strong scattering signal at the edges that can be well observed in the darkfield image (Fig. 3.55 (c)). Enhanced visibility of the cellular, filamentous structures is provided by horizontal and vertical differential phase contrast (Figs. 3.55 (d)-(e)).

Such a long, single filament was chosen to perform the ptychographic method, as shown by a reconstructed density map (Fig. 3.56 (a)) of two crossing SOX strands. The color code is already adapted to the projected electron density $\sigma_{e}$ and projected mass density $\sigma_{m}$. The visibly improved resolution reveals dense regions that are only barely visible in the STXM images and optical light micrograph. Importantly, the contrast yields a quantitative measure of the projected local mass density. For instance, the average over a homogeneous region of the filament yields $\left.<\sigma_{m}\right\rangle=0.033 \mathrm{mg} / \mathrm{cm}^{2}$ after background subtraction (region of background and SOX are indicated by white rectangles and black arrows in Fig. 3.56 (a)). In addition, two line regions were extracted from the density map (dashed red lines in Fig. 3.56 (a)) and are drawn in Figs. 3.56 (b), (c). The first line scan (b) includes a dense region right at the beginning of about $\sigma_{m} \approx 0.065 \mathrm{mg} / \mathrm{cm}^{2}$ after subtracting a background of $0.02 \mathrm{mg} / \mathrm{cm}^{2}$. The material corresponding to the line scan (c) appears with $0.08 \mathrm{mg} / \mathrm{cm}^{2}$ (after background correction) slightly denser. For completeness, the reconstructed projected absorption $\sigma_{\mu}$ is shown in Fig. 3.56 (d). In terms of resolution, it is clearly not as good as the density map of Fig. 3.56 (a), but clearly better than the transmission map in Fig. 3.55 (b). Dense regions such as occurring in (b) can be seen and show a comparably high absorption. 
a

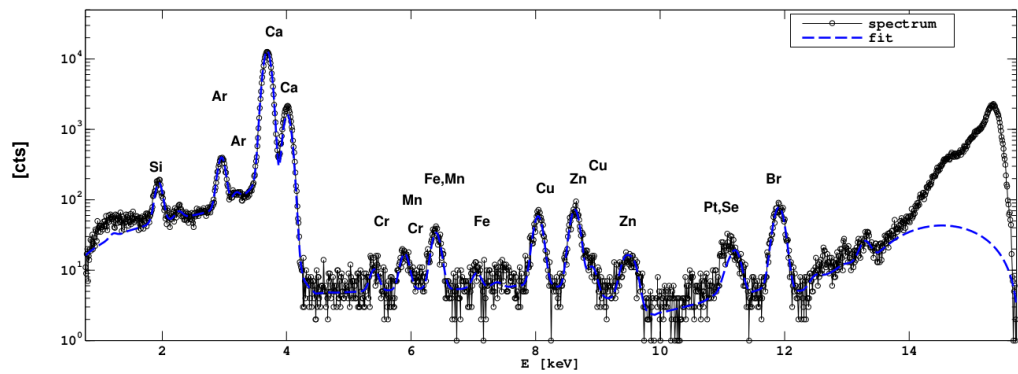

b
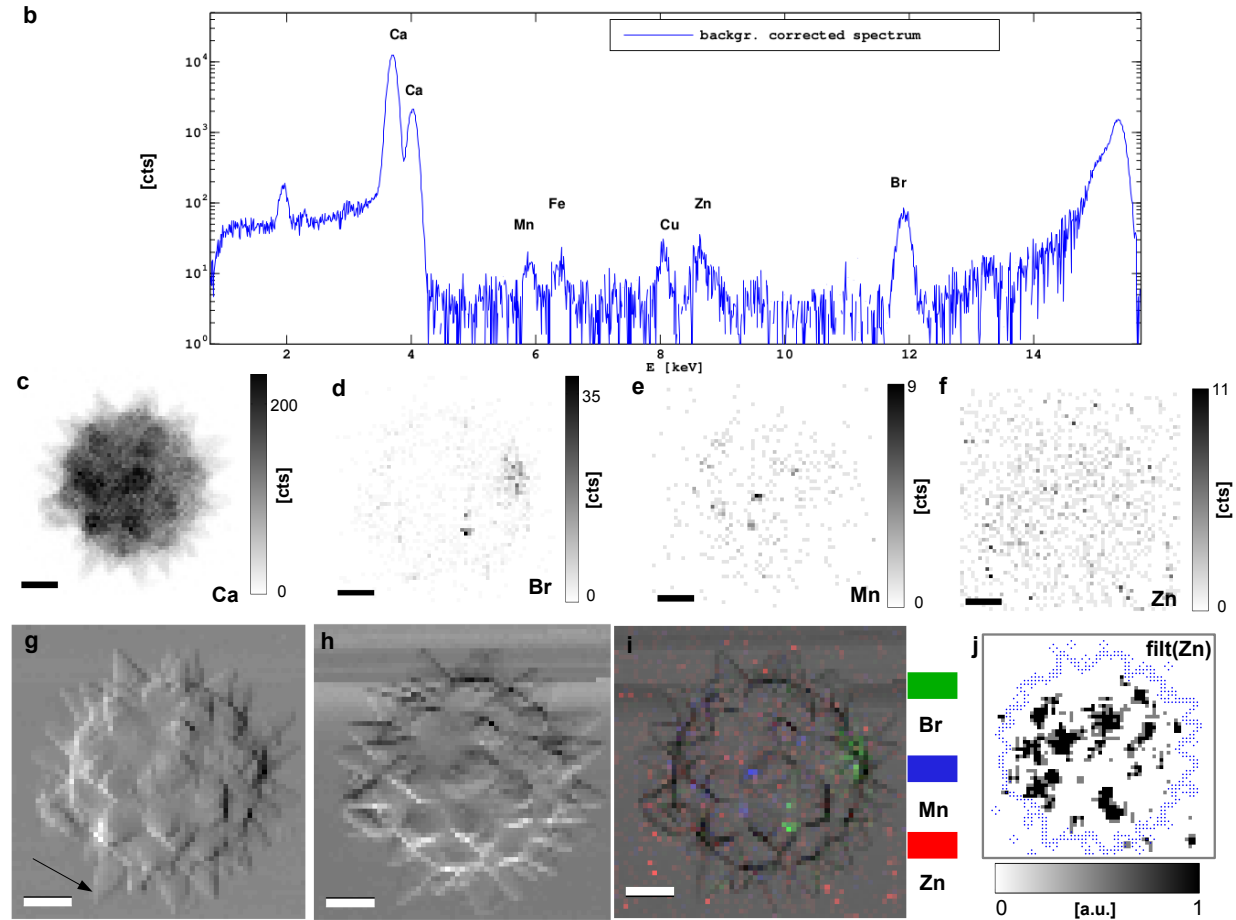

Figure 3.52: (a) summed spectrum of the scan $\mathrm{S} 1$ on $\mathrm{CaCO}_{3}$ fractions of the basal skeleton of $A$. willeyana presented in (c)-(i). The fit used to identify elements in the data is shown as dashed blue line. (b) spectrum as in (a) but background-subtracted. (c)-(f) fluorescence maps of the calcium, bromine, manganese, and zinc emission lines, respectively. (g), (h) differential phase contrast in the horizontal and vertical direction. The arrow in $(\mathrm{g})$ indicates the subregion with a single spike, which was scanned at higher resolution (cf. Fig. 3.53). (i) overlay image created by a combined differential phase contrast image of $(\mathrm{g})$ and $(\mathrm{h})$ (radial direction) and fluorescence channels (d)-(f). (j) shows the median filtered $\mathrm{Zn}$ distribution of (f). The blue dots indicate the shape of the spherulite. Scale bars in (c)-(i) denote $5 \mu \mathrm{m}$. 


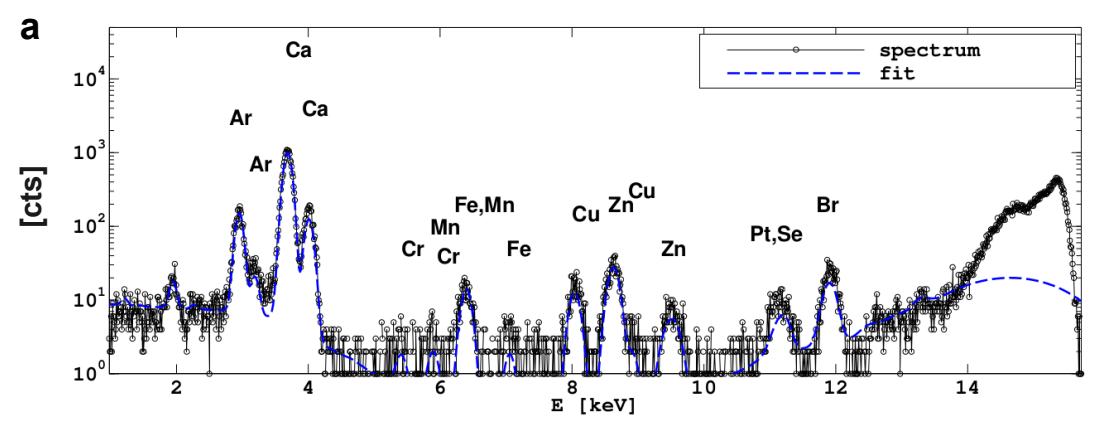

b
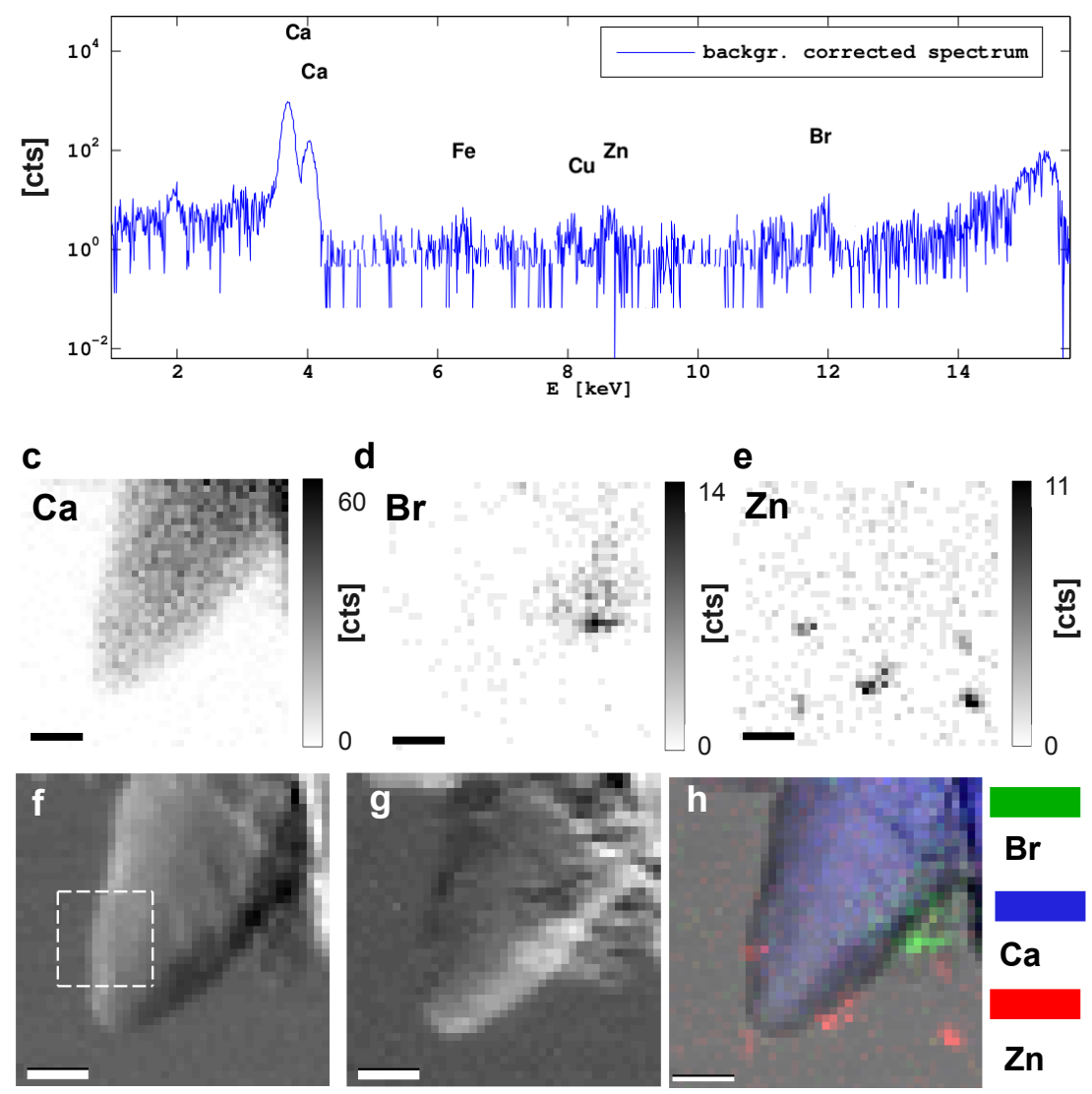

Figure 3.53: Scan S2 covering an area that is indicated by a black arrow in figure 3.52 (g). (a) summed fluorescence spectrum. (b) spectrum as in (a) but backgroundsubtracted. (c) calcium, (d) bromine and (e) zinc channel. (f) and (g) differential phase contrast in the horizontal and vertical direction, respectively. The dashed white frame in (f) indicates scan S3 as presented in figure 3.54 (h) overlay image created by a combined differential phase contrast image of $(\mathrm{f})$ and $(\mathrm{g})$ (radial direction) and fluorescence channels (c)-(e). Scale bars in (c) - (h) denote $1 \mu \mathrm{m}$. 

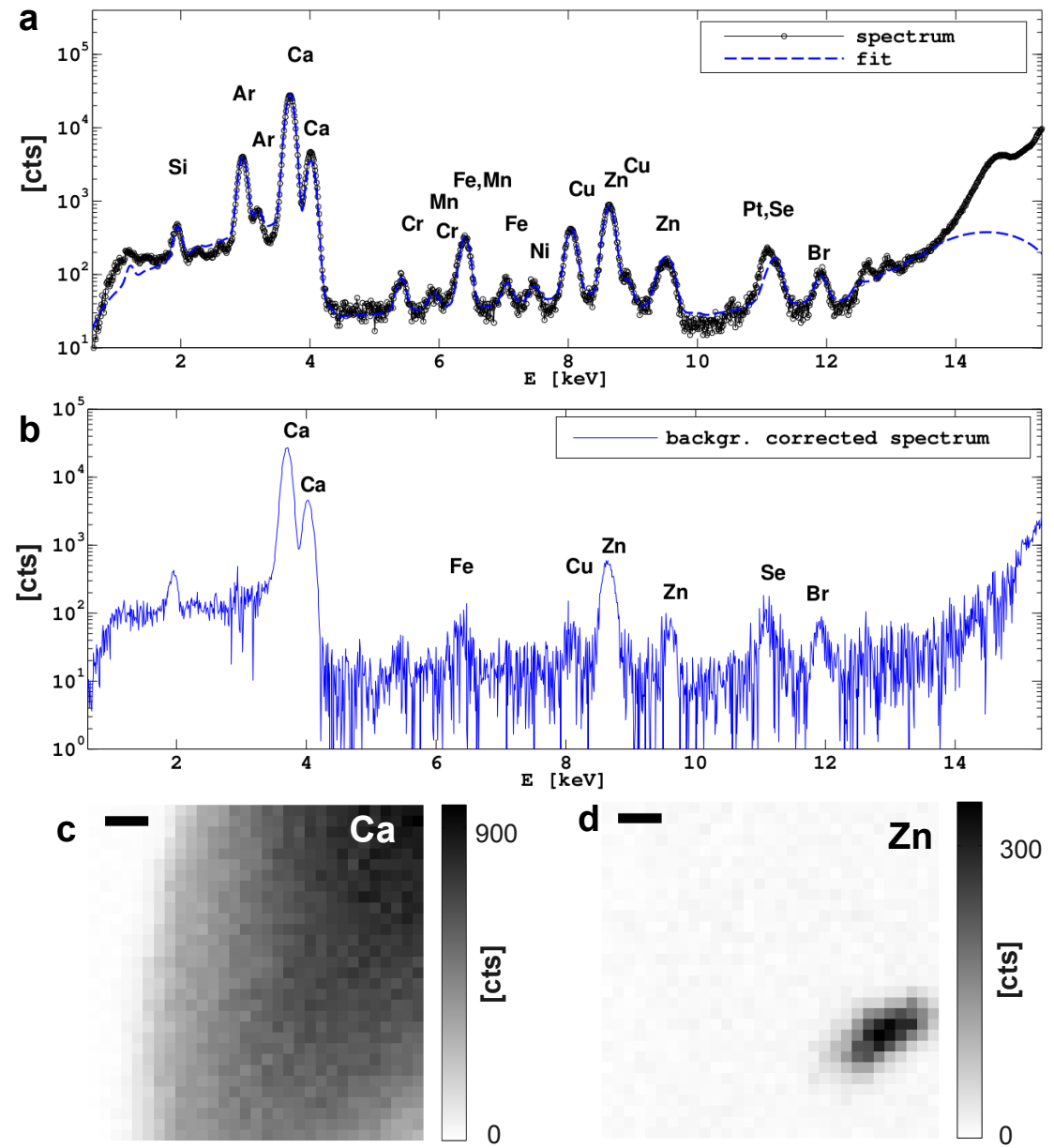

Figure 3.54: Scan S3 (cf. dashed white frame in Fig. 3.53 (f)). (a) summed fluorescence spectrum corresponding to the scan shown in (c), (d). (b) spectrum as in (a) but background-subtracted. In addition to the calcium peaks, zinc peaks can be observed. (c) calcium channel. (d) zinc channel. Scale bars in (c) - (d) denote $200 \mathrm{~nm}$. 

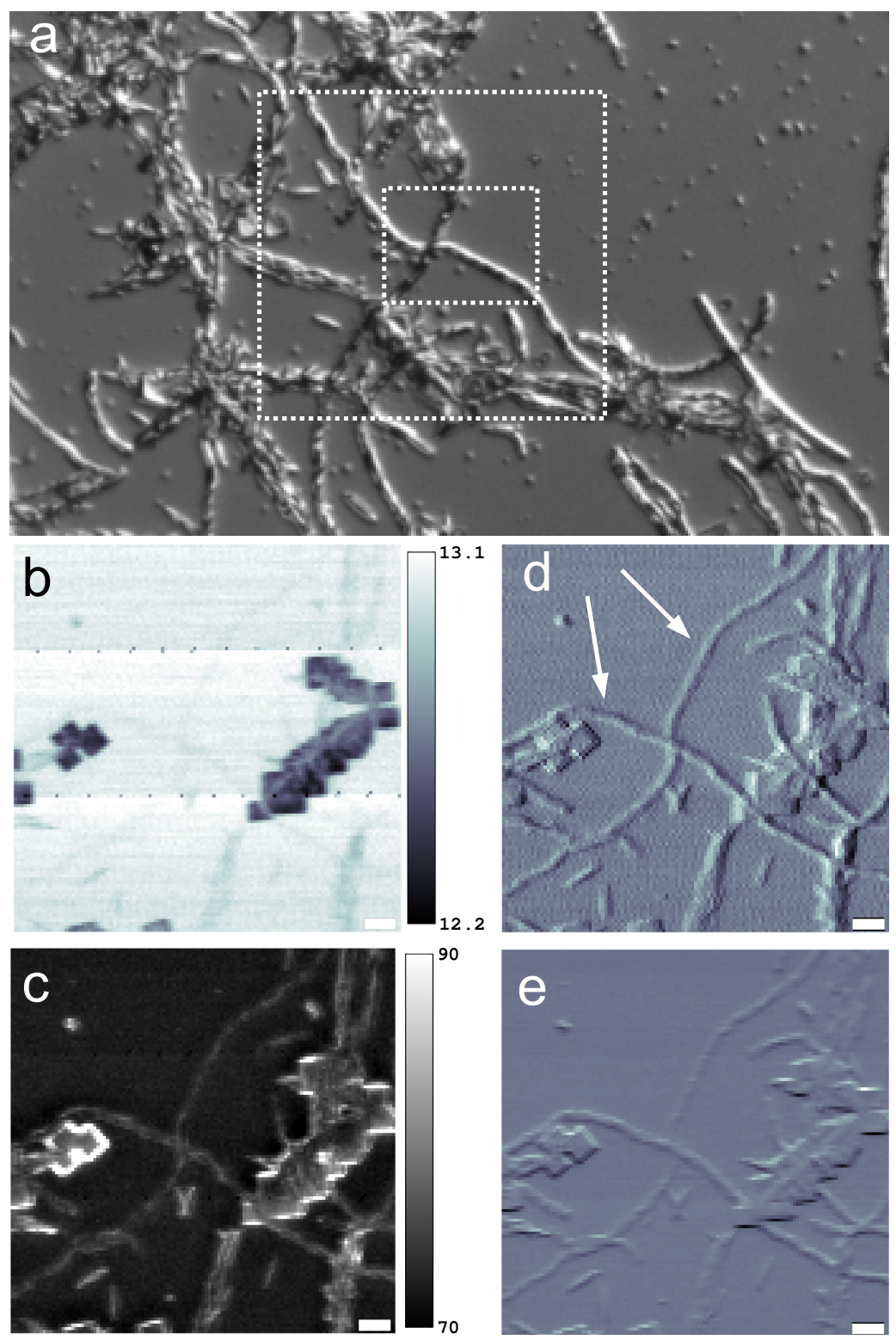

Figure 3.55: (a) shows an optical light micrograph (DIC) of the sample of SOX bacteria that is shown in (b)-(e) and figure 3.56 (rotated clockwise by $90^{\circ}$ ). The scanned area in (b)-(e) is indicated by the outermost dashed white lines. The inner dashed white lines sketch the area that was scanned with the ptychographic method (Fig. 3.56). (b)-(e) STXM contrasts of SOX bacteria: (b) transmission quantifies the transmitted photons at each point (colorbar in units of $10^{6}$ photons), (c) darkfield quantifies the ratio of scattered to unscattered photons (colorbar in units of $10^{3}$ ), (d) differential phase contrast in horizontal direction and (e) differential phase contrast in vertical direction. White arrows in (d) indicate bacterial filaments. Scale bars denote $5 \mu \mathrm{m}$ in all images. 

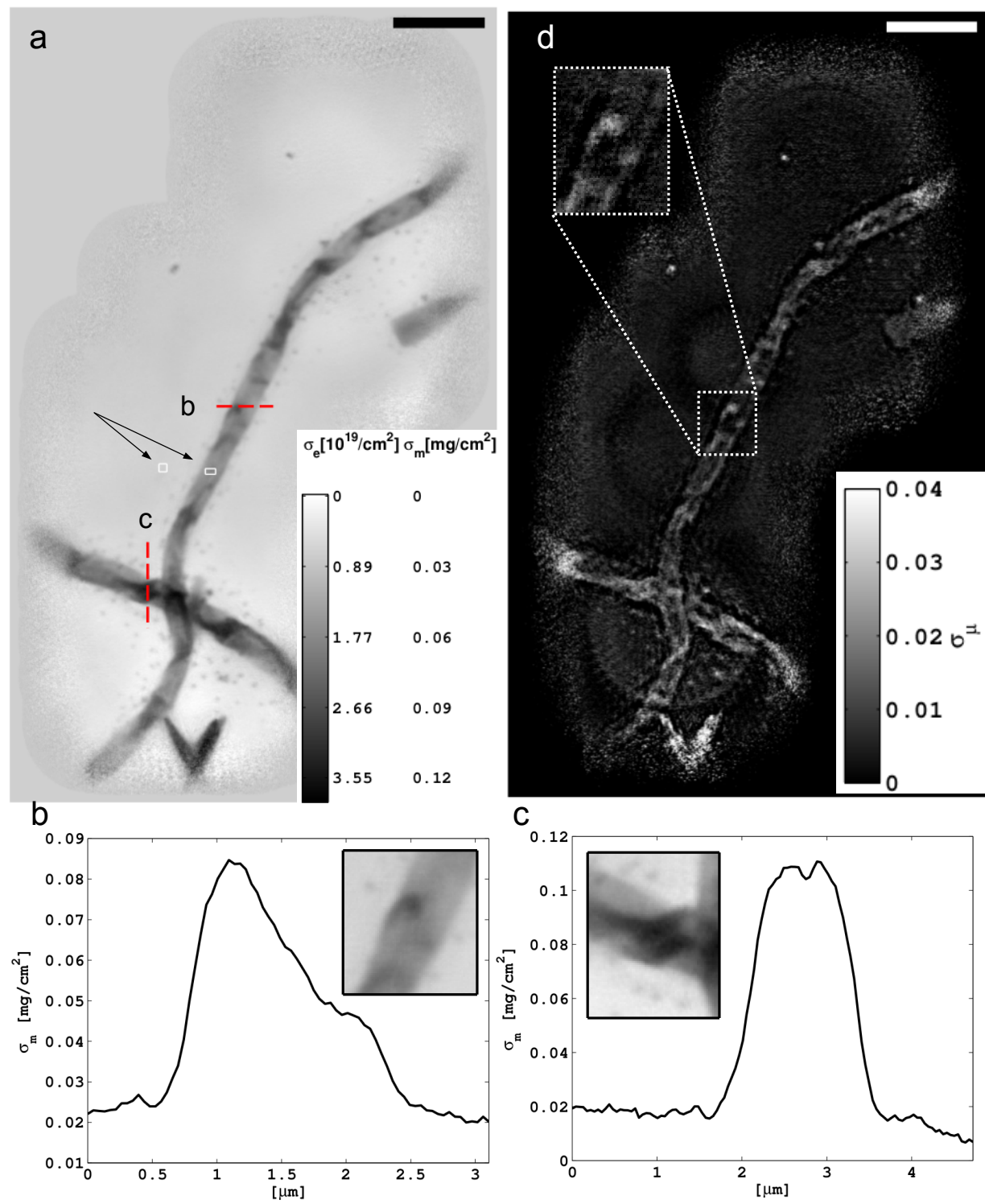

Figure 3.56: (a) The image presents the ptychograpic phase which quantifies the projected electron density $\sigma_{e}$ and projected mass density $\sigma_{m}$ of the scanned area showing two bacterial filaments of SOX. A homogeneous region of the cells yields a projected mass density of $\left\langle\sigma_{m}\right\rangle=0.033 \mathrm{mg} / \mathrm{cm}^{2}$ after subtracting the background (averaged regions are indicated by black arrows and white rectangles). (b) and (c) show lines through dense cellular material as indicated by dashed red lines on the density map in (a). Insets show magnified images of the regions of interest. (d) presents the projected absorption map $\sigma_{\mu}$ that corresponds to the density map (a). Inset shows magnified image of the region corresponding to (b). Scale bars denote $5 \mu \mathrm{m}$. 


\subsubsection{Details \& Results: SEM-EDX Analysis}

The Energy Dispersive X-ray spectroscopy (EDX) was carried out on the sample of SOX bacteria using the scanning electron microscope NovaNano SEM 650 (FEI, USA) after the X-ray analysis ${ }^{23}$. The $\mathrm{X}$-ray spectra were recorded with the silicon drift detector X-Max (Oxford, Uk; $80 \mathrm{~mm}^{2}$ active area). In order to make the sample compatible with SEM imaging, it was sputtered with a thin layer of carbon (nominal thickness $\sim 15 \mathrm{~nm}$ ). Although, carbon yields comparably low contrast and is prone to radiation damage it is well suited for this EDX analysis where the radiation from the sulfur edges needs to be distinguished from the background.

SEM images have been obtained from the same region which has been ptychographically analysed. For instance, the ptychographic reconstruction has been manually aligned with the SEM image as can be seen on the inset of Fig. 3.57 The agreement between both data is reasonable, given the contrast of the SEM image.

The EDX analysis was carried out at 10 different positions using a counting time of $60 \mathrm{~s}$ and an electron voltage of $15 \mathrm{kV}$. A SEM image taken afterwards clearly reveals measurement regions on the sample (cf. lower image in figure 3.58). Parts of the spectra are presented in the upper part of figure 3.58. The analysis with the Inca Software (Oxford, UK) shows a small sulfur signal that can be tentatively linked to the cells (8) or material from the cells close-by (1)-(3) as indicated by white arrow heads.

\footnotetext{
${ }^{23}$ I thank Matthias Hahn for helping me with the SEM-EDX analysis
} 


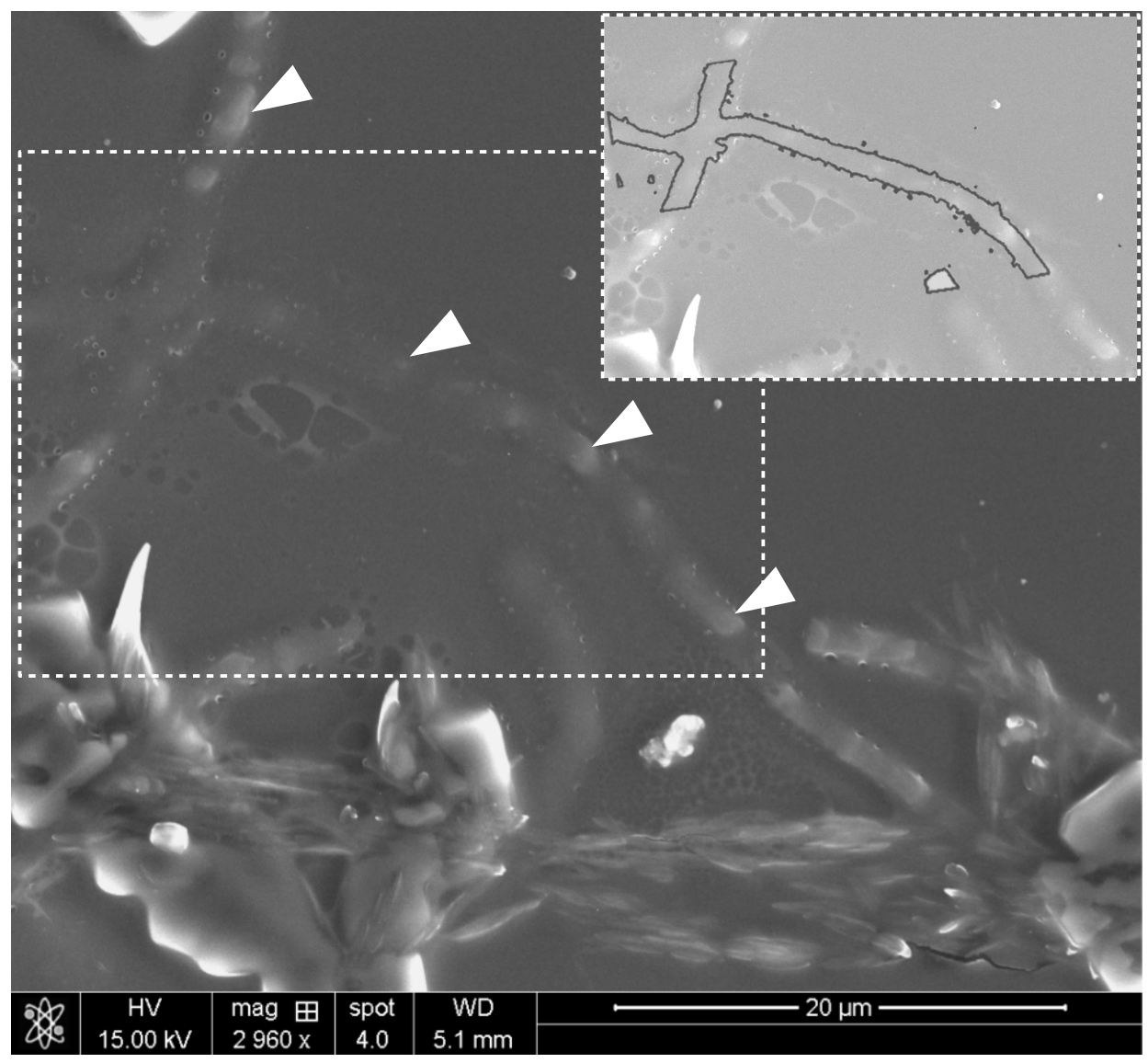

Figure 3.57: SEM image of the region of the sample that has been analysed with ptychography. The white arrow heads indicate cellular inclusions appearing in the SEM image. The inset illustrates the region that has been ptychographically reconstructed (dashed, white frame). The cell boundaries (black lines) from the reconstruction of Fig. 3.56 (a) are overlayed on top of a part of the SEM image. The SEM image has been taken after the X-ray experiment. 
(1)

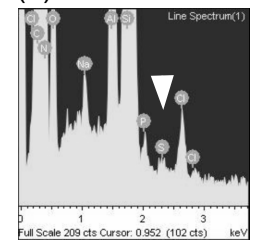

(6)

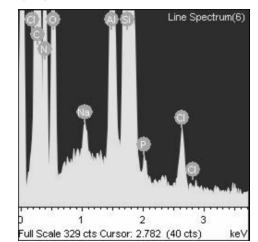

(2)

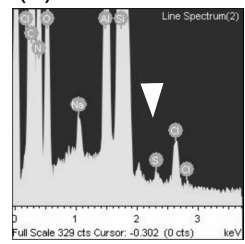

(7)

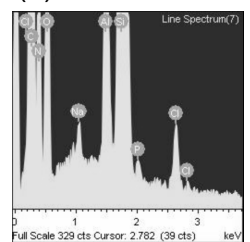

(3)

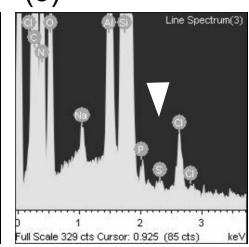

(8)

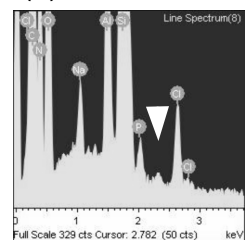

(4)

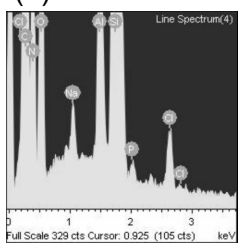

(9)

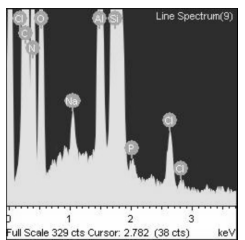

(5)

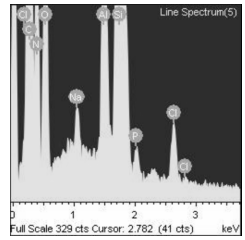

(10)

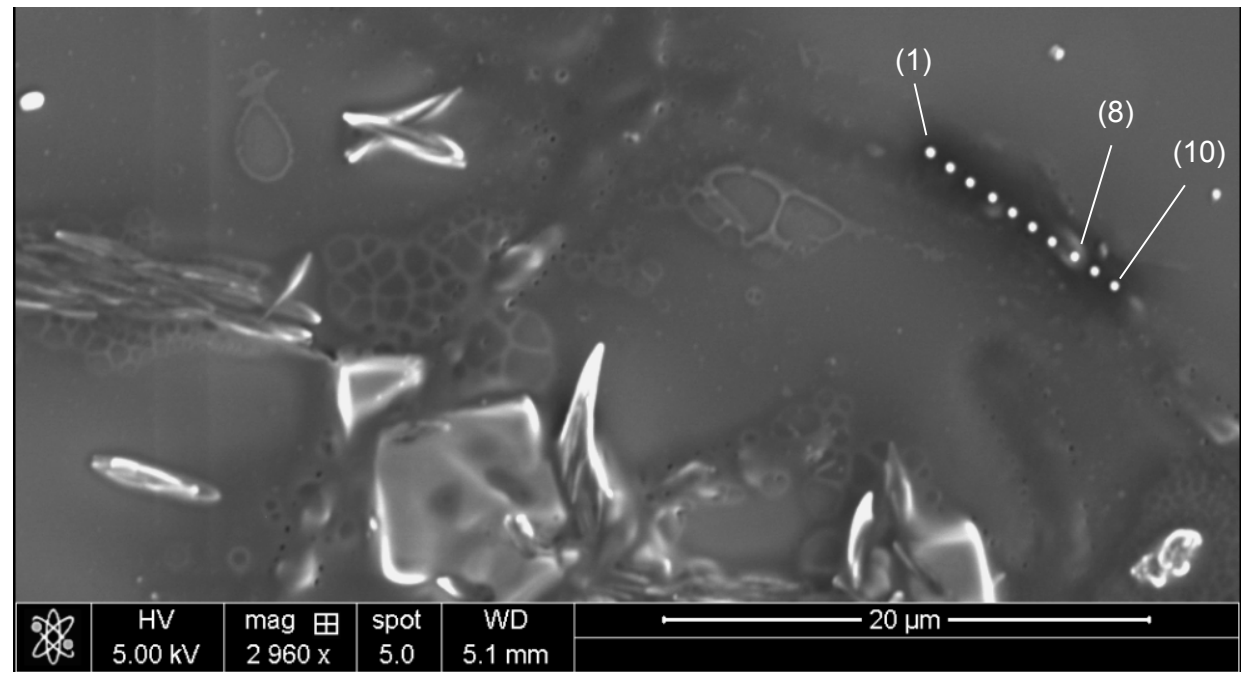

Figure 3.58: (1)-(10) EDX analysis $(15 \mathrm{kV})$ at single points of the sample with SOX bacteria. Locations (1)-(3), close to the cellular filament, clearly show a small sulfur contribution as indicated (white arrow heads). The sulfur signal cannot be well discriminated from the background at the other points but there seems to be still some signal as indicated in (8) by a white arrow head. The SEM image below which was taken after the analysis reveals the measurement points due to damage from the electron beam. Note that (8) lies on top of a cellular feature appearing bright in the SEM image and dark in the phase reconstruction (Fig. 3.56 (a)). The ordering of the EDX spectra is linear according to the labels. 


\subsubsection{Discussion \& Conclusion}

The solid aragonite skeleton of the coralline demosponge Astrosclera willeyana is steadily enlarged by large vesicle cells through the deposition of micron-sized primary aragonitic skeletal elements (spherulites) [145,338. Before their inclusion into the skeleton, spherulites such as presented in figure 3.52 , gradually increase in size and finally, fuse with the growing skeleton $[80,247,338$. The study of these intracellularly formed $\mathrm{CaCO}_{3}$ spherulites shows that the combination of fluorescence and STXM data can be used to locate minimal elemental concentrations of zinc, bromine and manganese. The best visualisation of the sample was achieved by differential phase contrast (darkfield and transmission are not presented). This is in good agreement with the expectation because the theoretical absorption length of $\sim 530 \mu \mathrm{m}$ of $\mathrm{CaCO}_{3}$ for $15.25 \mathrm{keV}$ photons [125] predicts a low absorption contrast. The fluorescence analysis on a single spike shows that bromine forms a thin layer close to the surface of the spherulite. Besides calcium and bromine, predominant elements located near and on a spherulite of $A$. willeyana were zinc, iron and copper (Figs. 3.53 (b), 3.54 (b)). Zinc, for instance, is bound within the metalloenzyme carbonic anhydrase (CA), catalysing the reversible reaction between carbon dioxide and water and bicarbonate and protons being important to e.g. the process of biocalcification [127]. In A. willeyana $\alpha$-CAs such as Astrosclerin-1, 2 and 3 are responsible for catalysing the chemical reaction $\mathrm{CO}_{2}+\mathrm{H}_{2} \mathrm{O} \leftrightarrows \mathrm{HCO}_{3}^{-}+\mathrm{H}^{+}$which represents the source of $\mathrm{HCO}_{3}^{-}$necessary for building a $\mathrm{CaCO}_{3}$ skeleton |145. Hence, the zinc signal in the data is likely due to remainders of the CAs in the sample material. Furthermore, the results indicate that part of the zinc signal originates from the spherulite or its surface which coincides well with the growth process of the spherulites and the expected occurrences of CAs. According to figure 3.53 (h), fluorescence analyses revealed a zinc signal more or less bound to the top of a spherulite spike, suggesting any remains of the metalloenzyme. At the same time, a clear bromine signal which is known to have some inhibiting effects to Astrosclerin 220 is detected at the base of the spike. The simultaneous, but spatially separated occurrence of catalysing and inhibiting factors may be indicative of the terminal stage of spherulite calcification.

The accessible fluorescence lines in all scans are limited by the X-ray energy of $15.25 \mathrm{keV}$, excluding e.g. fluorescence emission of strontium (K-shell electron binding energy of $16.11 \mathrm{keV}$ ), which is typically observed in calcified samples. In addition, at the high energy end all considered spectra are dominated by Compton and Rayleigh scattering, and in the low energy region two argon peaks arise due to the scattering of X-rays in air. X-ray fluorescence lines below the first argon peak such as $\mathrm{S}\left(\mathrm{K}_{\alpha}\right)$ are suppressed due to absorption from air. In the future, improvements for the detection of fluorescence lines could be achieved by slightly increasing the X-ray photon energy and an optimisation of the set-up of the fluorescence detector. In order to further support the outlined arguments for the linkage between metalloenzymes and fluorescence signals future data on reference samples could be helpful. 
The STXM images (Figs. 3.55 (b)-(d)) of the sample of sulfur-oxidizing communities show filamentous strands of bacteria and crystalline material that is supposedly a remainder of phosphate buffered saline from initial sample treatments. The ptychographic imaging method was successfully applied on filamentous sulfuroxidizing communities by scanning along a single bacterial filament. The projected mass density map which corresponds to phase contrast yields the best imaging quality. The result agrees well with the expectation as absorption contrast from a $1-2 \mu \mathrm{m}$ thick layer of biological material containing mostly proteins is almost negligible. One advantage of the ptychographic method is its capability of yielding super-resolution, i.e. resolution below the beam size. Here, the resolution of the ptychographic reconstruction is around $50 \mathrm{~nm}$ [330 whereas the STXM images (Fig. 3.55) are limited by the beam size of $\sim 500 \mathrm{~nm}$. It is remarkable that the improvement in resolution does not limit the field of view here. In this experiment ptychographic imaging is achieved over an area of about $20 \mu \mathrm{m} \times 40 \mu \mathrm{m}$ of the sample. Experimentally limiting factors such as changes of the illuminating beam and positional uncertainties have been effectively overcome. The potential of this ptychographical scanning scheme is also illustrated in a very recent work using a similar approach 112 . In comparison to the other datasets, the scan with the highest possible resolution of about $80 \mathrm{~nm}$ (the beam size) is S3, which only covers an area of about $2 \mu \mathrm{m} \times 2 \mu \mathrm{m}$. Importantly, the ptychographic data could be used to quantify the mass density of the cellular material and cellular features on the level of single, unsliced and unstained cells. Furthermore, the map of the projected mass density reveals dense regions that may be attributed to cellular inclusions. For instance, the dense region in the line scan (b) (Fig. 3.56) yields $\sigma_{m} \approx 0.032 \mathrm{mg} / \mathrm{cm}^{2}$ after subtracting the measured density $<\sigma_{m}>0.033 \mathrm{mg} / \mathrm{cm}^{2}$ of supposedly pure cellular material. Attributing this feature to sulfur and taking literature values for the mass density $\left(\rho_{S}=2.07 \mathrm{~g} / \mathrm{cm}^{3}\right)$ one obtains a reasonable thickness of about $155 \mathrm{~nm}$. Hence, the dense region may be attributed to such intracellular sulfur inclusions, which are transiently formed in the process of oxidizing reduced sulfur compounds like sulfides and thio-sulfate into sulfate 54,185 . The SEM-EDX analysis revealed a small sulfur signal which may be tentatively linked to the sample. The signal could be detected next to and on a bacterial filament. The former signal may arise from the small particles of sample material that can be seen in the ptychographic reconstruction next to the filaments. The latter signal may arise from a large cellular inclusion (position (8)). A SOX bacterium similar to the filamentous morphotypes presented here is Thiothrix sp., where the intracellular storage of sulfur depends on the presence of thiosulphate, nitrate and oxygen. Such filaments may loose sulfur globules (produced under aerobic conditions from thiosulphate) when exposed to anaerobic conditions 215 . An appealing application is the combination of ptychography with the simultaneous recording of fluorescence data ${ }^{24}$, which opens the possibility to combine the measurements of mass density with elemental mapping 265 . The ptychographically obtained information about the illuminating wave-field can also be used by

\footnotetext{
${ }^{24}$ The used set-up at the cSAXS simply did not include a fluorescence detector.
} 
deconvolution methods to increase the resolution of the fluorescence data [315]. Moreover, one way to obtain more reliable estimates of the full 3D electron and mass density distribution of this sample would be to combine the ptychographic imaging scheme with tomography (e.g. $[61,330 \mid$ ). A tomographic dataset would also be more suitable for analysing inclusions.

In conclusion, X-ray experiments on two different geobiological sample systems at two different X-ray scanning microscopes have been performed. In particular, elements such as calcium and zinc that take part directly or are involved indirectly through metalloenzymes in the underlying relevant chemical reactions could be identified and mapped by combining different X-ray imaging modes. In future, the ptychographic method may become relevant as a high resolution, large field of view imaging technique providing quantitative contrast. The results will be helpful to better understand the studied sample systems and processes such as biocalcification. 


\subsection{Semi-Transparent Central Stop in High-Resolution X- Ray Ptychography Using Kirkpatrick-Baez Focusing}

A Ptychographic Coherent X-ray Diffractive Imaging (PCDI) experiment using $7.9 \mathrm{keV}$ X-rays and Kirkpatrick-Baez focusing mirrors (KB) was carried out. In order to achieve high resolution images one has to invest sufficiently high doses [141]. In PCDI, the challenge is to simultaneously record the very intense primary beam and the quickly decaying scattering signal $\left(\propto q^{-\alpha}\right.$, with $\left.\alpha \approx 4\right)$. By introducing a Semi-Transparent Central Stop (STCS) in front of the detector the dynamic range on the detector is increased by $\sim 4$ orders of magnitude. The feasibility of this experimental scheme for PCDI applications with a resolution below $10 \mathrm{~nm}$ is demonstrated. The results are compared with reference data and an increase of resolution by a factor of two is obtained while the deviation of the reconstructed phase map from the reference is below $1 \%$.

In view of the dynamic range problem, PCDI in combination with focused (divergent) beams is most suitable for high resolution imaging of extended specimen: The fluence on the sample is maximised in the focus while the full intensity is spread over a comparably large area of the detector due to the divergence of the beam. First results using divergent beams have been reported, including nanofocusing refractive X-ray lenses $[134,265 \mid 266$, Fresnel zone plates $\mid 143,312,330$ and Kirkpatrick-Baez (KB) mirrors $[98,143,286,287$. KB mirrors are attractive focusing devices due to their high efficiency at sub $100 \mathrm{~nm}$ focusing while providing long working distances of hundreds of millimetres $130,189,199-201$. However, efficient KB mirror optics in combination with the high coherent flux provided by modern synchrotrons such as PETRA III result in data that cannot be processed by current photon counting detector technologies. Recently, Liu 174 suggested to overcome the high dynamic range problem by using a fully absorbing beamstop. Their numerical simulations show that the missing data problem can be partly solved by PCDI. In addition, the problem has previously been approached by patching data recorded with a fully absorbing beamstop with data taken where the central part has been attenuated $|286,287|$. Here, the high dynamic range problem is approached by the use of a highly attenuating single Semi-Transparent Central Stop without the need of patching two diffraction patterns and the additional recording at each scan point. The reliability of this scheme is addressed, guidelines for designing the STCS are given and it is outlined how it is best introduced by making use of a KB set-up. The STCS data is compared with data taken without STCS in order to validate the quantitativeness of the ptychographic reconstructions. This section closes by an analysis about the increase in resolution and a brief discussion about future improvements in STCS fabrications.

The section here is partly based on results published in 332 . The design and fabrication by Malte Vaßholz of the W-STCS being used here is gratefully acknowledged. In his Bachelor thesis other approaches to the re-scaling problem of the STCS data were analysed $\mid 310$. 


\subsubsection{Experimental Set-up}

The ptychographic imaging experiment was carried out at the coherent nanofocus endstation GINIX (Göttingen Instrument for Nano-Imaging with X-rays) of the P10 coherence beamline of the PETRA III storage ring located at DESY, Hamburg. The photon energy was defined by a Si(111) monochromator to be $7.9 \mathrm{keV}$. The focusing set-up consists of two pairs of beam-defining slits and Siattenuation foils in front of two elliptical X-ray mirrors in the Kirkpatrick-Baez (KB) geometry (for a schematic see Fig. 3.59 (a)). The Pd coated vertical mirror (WinlightX) and the horizontal mirror (JTEC) were designed for $4.05 \mathrm{mrad}$ inc. angle (centre) and focal distances of $302 \mathrm{~mm}$ and $200 \mathrm{~mm}$, respectively (for details see $[152-154$ ). A $5 \mu \mathrm{m}$ diameter W-pinhole (foil thickness $40 \mu \mathrm{m}$ ) was placed at ca. $1 \mathrm{~mm}$ in front of the $\mathrm{KB}$ focus in order to guarantee the oversampling condition for ptychographic phasing 195 by effectively absorbing tails of the KB beam [98]. In the standard mode of ptychography the sample is scanned along a rectangular grid of points in a plane of the $\mathrm{KB}$ focus and the resulting diffraction patterns are collected by the photon counting pixel detector (pixel size $172 \mu \mathrm{m} \times$ $172 \mu \mathrm{m}$ ) PILATUS 300K (DETCRIS) placed about $5.1 \mathrm{~m}$ behind the focal plane. Absorption and background originating from air scattering are reduced by an evacuated flight-tube between the sample and the detector. Note that the beam usually needs to be strongly attenuated in order to prevent damage of the detection device. The maximum count rate of the PILATUS of about $2 \times 10^{6}$ phot $/ \mathrm{s} \mid 306$ is exceeded by the available full coherent flux of approx. $10^{11} \mathrm{phot} / \mathrm{s}[258]$. The set-up is thus expanded by introducing the Semi-Transparent Central Stop in the flight-tube at ca. $15 \mathrm{~cm}$ in front of the photon detector ((7) in Fig. 3.59 (a)). The STCS consists of a rectangular $25 \mu \mathrm{m}$ thick W-foil (ChemPur) cut to a size of $2.2 \mathrm{~mm} \times 2.0 \mathrm{~mm}$ by wire-cut electric discharge machining. The STCS is fixed on top of a polymide-foil and can be moved in and out of the beam in front of the detector. The STCSs' theoretical transmission is $0.24602 \times 10^{-3}$ at $7.9 \mathrm{keV}$ photon energy 125 .

From the variety of available materials that can be used for STCS applications tungsten is favoured for several reasons. Firstly, tungsten yields a comparably high attenuation in comparison to other materials such as silicon. This is advantageous as thick layers of material are likely to produce a varying attenuation at the boundaries due to a slight tilt with respect to the optical axis. Secondly, the main X-ray fluorescence emission lines are well above or below the energy threshold of the PILATUS 300K at $7.9 \mathrm{keV}$ photon energy. Moreover, tungsten foils are commercially available in the favoured dimensions of $\mathrm{mm} \times \mathrm{mm} \times \mu \mathrm{m}$ and can be easily processed. This is particularly helpful in synchrotron applications, where the beam-shape of the experiment cannot always be anticipated with an accuracy in the range of the pixel dimension of the detector before the experiment. The problem of designing the right dimensions for the STCS can thus be circumvented by fabricating a set of different sizes. Importantly, the STCS allows to increase the photon flux on the sample by two orders of magnitude without saturating the photon counting device. 

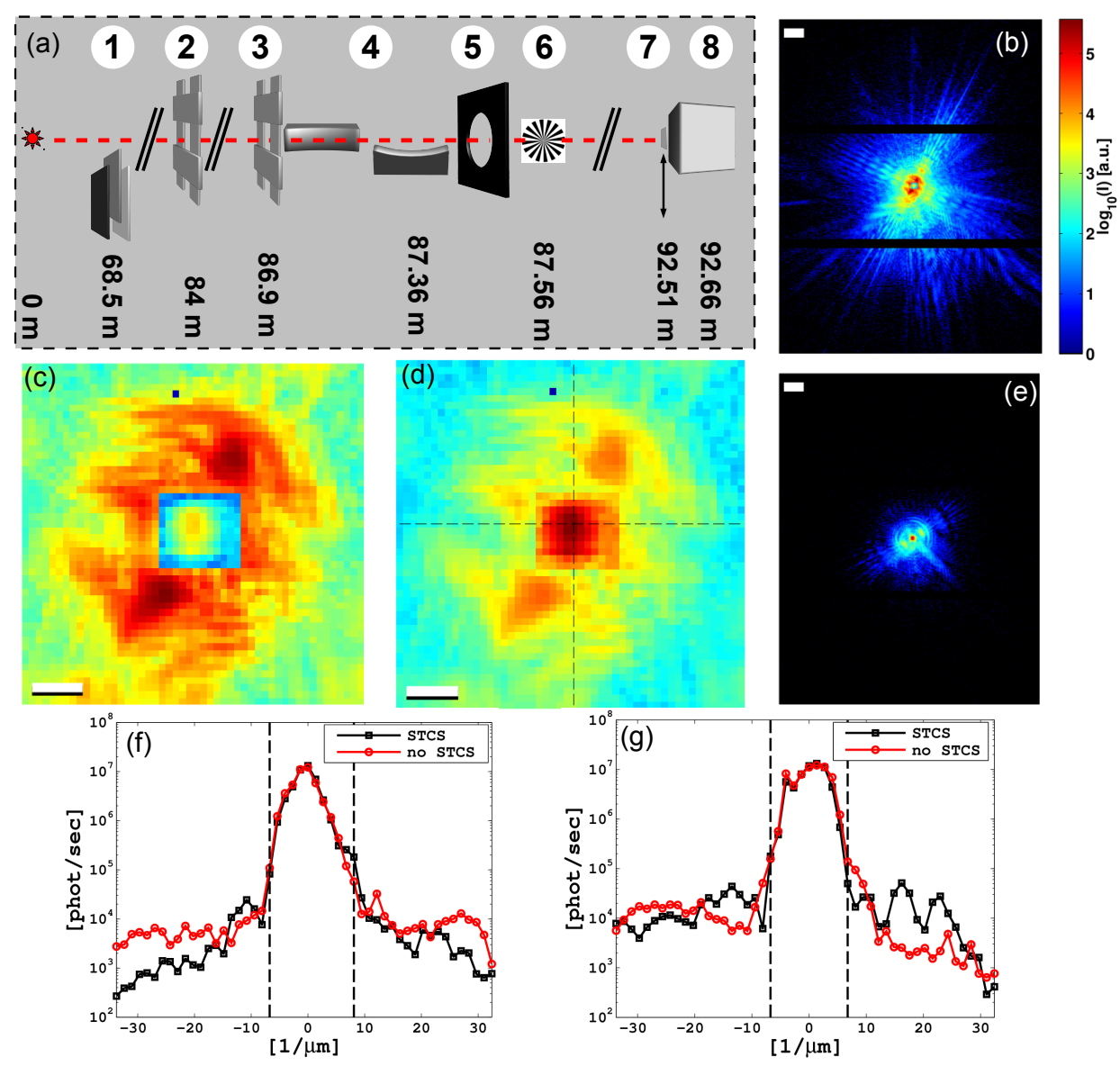

Figure 3.59: (a) Schematic of the set-up: (1) Si-attenuation foils, (2) slits S1, (3) slits S2, (4) focusing optics (Kirkpatrick-Baez mirrors), (5) pinhole, (6) focal region with the resolution chart sample, (7) semi-transparent central stop (STCS), (8) photon counting detector. (b) shows a diffraction pattern taken with STCS and $166.8 \mu \mathrm{m} \mathrm{Si}-$ attenuation foils (1). (c), (d) show inner part from (b) without and with rescaling with respect of the area attenuated by the STCS (7), respectively. (e) shows diffraction pattern taken without STCS but with $444.8 \mu \mathrm{m}$ Si-attenuation foils (1). (f), (g) show line profiles through the rescaled diffraction pattern shown in (d) (indicated by black, dashed lines in (d)) in the horizontal and vertical direction (solid black lines), respectively. Up-scaled line profiles (scaling factor $=8.3 \cdot 10^{1} / 1.3=63.85$ ) through a diffraction pattern taken without the STCS are shown as solid, red lines. The STCS region is indicated by black dashed lines in (f) and $(\mathrm{g})$. Scale bars denote $50 \mu \mathrm{m}^{-1}$ and $10 \mu \mathrm{m}^{-1}$ in (b), (e) and (c), (d), respectively. The exposure time is $1 \mathrm{~s}$ in (b) and (e). The colorbar in (e) is the same as in (b). 
Table 3.9: The table summarizes information on used slit settings.

\begin{tabular}{lcc}
\hline slits & horizontal gap $[\mu \mathrm{m}]$ & vertical gap $[\mu \mathrm{m}]$ \\
\hline S1 & 40 & 100 \\
S2 & 100 & 100 \\
\hline
\end{tabular}

Table 3.10: The table summarizes information on used Si-attenuation foils and attenuation with respect to the W-STCS in front of the detector. The measured photon flux on the sample is shown, too.

\begin{tabular}{lccc}
\hline Dataset & $\begin{array}{c}\text { Transmission } \\
\text { of Si-foils }\end{array}$ & $\begin{array}{c}\text { Transmission } \\
\text { of W-STCS }\end{array}$ & $\begin{array}{c}\text { photon flux } \\
\text { [phot/s] }\end{array}$ \\
\hline 1 & $8.3 \cdot 10^{-2}$ & $4.0068 \cdot 10^{-4}$ & $2.7 \cdot 10^{8}$ \\
2 & $1.3 \cdot 10^{-3}$ & 1 & $4.4 \cdot 10^{6}$ \\
\hline
\end{tabular}

A first ptychographic dataset (dataset 1) was collected on a Siemens star X-ray resolution chart $(500 \mathrm{~nm}$ thick layer of tantalum with finest feature size of $50 \mathrm{~nm}$; model ATN/XRESO-50HC, NTT-AT, Japan) using the W-STCS in front of the detector. An important feature of the GINIX versatility arises from the slits in front of the $\mathrm{KB}$ optics which can be used to change the numerical aperture of the mirrors. The slits can thus be used to change the focal size, the divergence of the beam and consequently, its imprint on the detector [98, 189]. An optimum match between the imprint of the beam and the area covered by the STCS was found by choosing a position of the slits where they slightly cut into the beam (cf. Tab. 3.9). At $7.9 \mathrm{keV}$ the STCS attenuates the central beam by ca. 4 orders of magnitude. An additional attenuation of the X-ray beam by approx. 1 order of magnitude (cf. Tab. 3.10) was necessary to avoid saturation of the detector. From the optimum scaling factor $\gamma$ of the STCS for the attenuated region of the detector, a photon flux of $2.7 \times 10^{8} \mathrm{phot} / \mathrm{s}$ was inferred (cf. Tab. 3.10). The ptychographic data consist of 441 scan points taken on a rectangular mesh with an uniform grid size of $150 \mathrm{~nm} \times 150 \mathrm{~nm}$, and it covers a scan-region of $3 \mu \mathrm{m} \times 3 \mu \mathrm{m}$. The exposure time of each grid point is one second. The total measurement time including movements of the sample was $\sim 9 \mathrm{~min}$.

Secondly, ptychographic data (dataset 2) were collected on the same specimen without attenuating the central beam in front of the detector necessitating a higher global attenuation of the full X-ray beam. The missing STCS attenuation was compensated by an attenuation of $\sim 10^{-3}$ through Si-attenuation foils in front of the KB optics (cf. Tab. 3.10). Thereby, the total photon flux available for ptychography was reduced to $4 \cdot 10^{6}$ phot/s. The scanned area was $4 \mu \mathrm{m} \times 4 \mu \mathrm{m}$ with a grid size of $160 \mathrm{~nm} \times 160 \mathrm{~nm}$. In total, 676 diffraction patterns were recorded using an exposure time of $1 \mathrm{~s}$ per scan point. The total measurement time including movements for scanning the sample was $\sim 15 \mathrm{~min}$. 
Table 3.11: The table shows the average phase shift (Ta versus no Ta) from the Siemens star test pattern as obtained through ptychographic phasing. For the case of using an STCS the dependence of different STCS scaling factors on the phase shift $\langle\Delta \Phi\rangle$ is listed.

\begin{tabular}{llc}
\hline Dataset & $\begin{array}{l}\text { STCS scaling } \\
\text { factor } \gamma\end{array}$ & $<\Delta \Phi>[\mathrm{rad}]$ \\
\hline 2 & 1 & -0.757 \\
1 & $5 \cdot 10^{2}$ & -1.341 \\
1 & $1 \cdot 10^{3}$ & -1.032 \\
1 & $2.496 \cdot 10^{3}$ & -0.755 \\
1 & $4.054 \cdot 10^{3}$ & -0.621 \\
\hline
\end{tabular}

\subsubsection{Details: Analysis}

The data were analysed using the 'ePIE' algorithm 183. The output of the algorithm are estimations of both the complex probe $\left.\overrightarrow{P(\vec{x}}-\vec{x}_{i}\right)$ and the object $O(\vec{x})$ being illuminated by the probe at positions $\vec{x}_{i}$. All phase reconstructions were obtained after 400 iterations as an average over the last 100 iterations and by using $\alpha=\beta=0.5$ (cf. $|183|)$. The sample plane was initialised as uniform amplitude with constant phase, whereas the probe was initiated as circular aperture of diameter $600 \mathrm{~nm}$ with constant amplitude and phase. A region of $460 \times 600$ pixels $(h \times v)$ around the central peak of the recorded diffraction patterns was used yielding a real space pixel size of $10.1 \mathrm{~nm} \times 7.8 \mathrm{~nm}$ in the horizontal and vertical direction, respectively. The insensitive horizontal stripes between adjacent detection modules of the PILATUS (cf. Fig. 3.59 (b)) were treated as zero intensity regions during the iterative reconstructions.

At first, the influence of different scaling factors for the attenuated central intensity of dataset 1 on the reconstruction was studied. Phase reconstructions corresponding to varying scaling factors $\gamma$ in the range of $10^{2}$ to $10^{7}$ are presented in Fig. 3.60. The theoretical scaling factor $\gamma=4.054 \times 10^{3}$ corresponding to the theoretical value of $25 \mu \mathrm{m}$ tungsten was also included. The phase reconstructions have been compared by calculating the relative phase shift of the Siemens star resolution chart (cf. Fig. 3.61). As a criterion for reconstruction quality the deviation of the phase from the average of the uniform regions, with and without tantalum, was evaluated. A phase histograms such as can be seen in Fig. 3.61 (a) was used to define the areas with and without Ta within a chosen rectangular region of the reconstructed phase maps by fitting a sum of two Gaussians to the histogram. It was found that the binary structure of the Siemens star is well reproduced if all phases lying in a $2 \mathrm{FWHM} \approx 4.7 \sigma$ interval (full width at half maximum) were included (cf. Fig. 3.61 (a)). The results of the calculated phase shifts $\langle\Delta \Phi\rangle$ are shown in Figs. 3.61 (b), (c) and Tab. 3.11. 


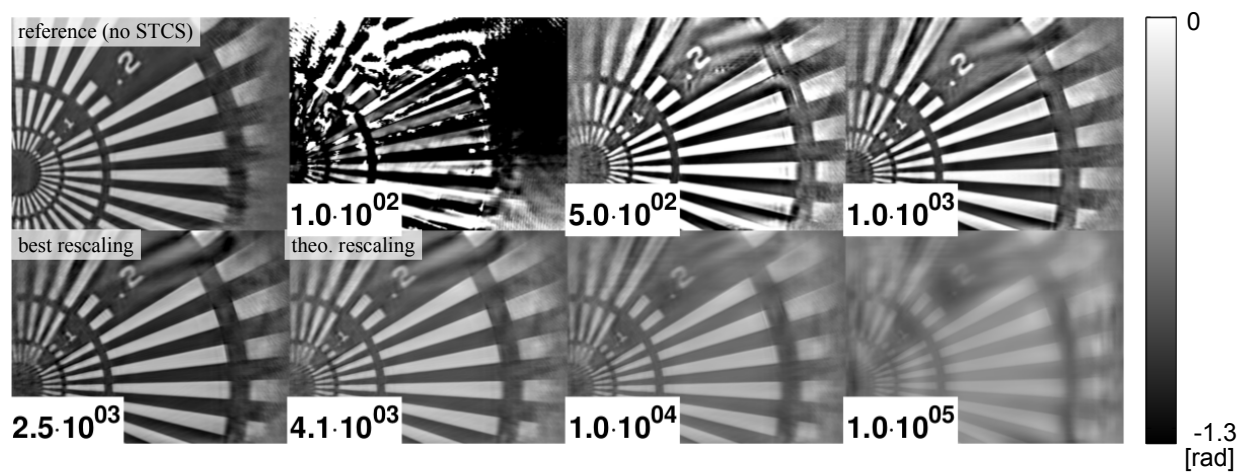

Figure 3.60: Phase reconstructions of dataset 1 assuming different STCS transmission values in comparison to the reference phase reconstruction of dataset 2 (without attenuation of the central beam) which is shown at the top left position. Corresponding scaling factors $\gamma(1 /$ transmission $)$ are shown as insets.
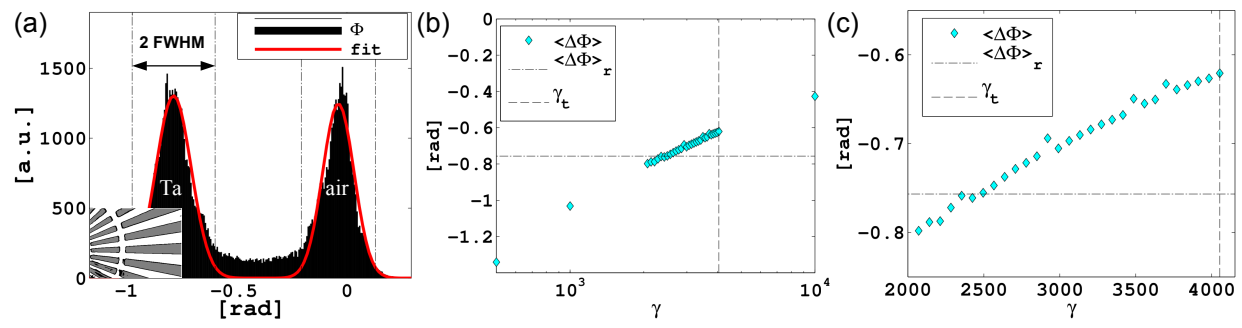

Figure 3.61: (a) shows phase histogram corresponding to dataset 2. The histogram contains only phases within the rectangular area depicted in the inset. A fit of a sum of two Gaussians to the histogram is drawn in red. The phase of Ta (or no Ta) is estimated as an average over one 2 FWHM interval (dash-dotted, black lines). The intervals correspond to the white and grey regions in the graph of the inset. (b) presents estimations of $\langle\Delta \Phi\rangle$ corresponding to dataset 1 for different values of $\gamma$ using 2 FWHM intervals of the histograms. The reference $\langle\Delta \Phi\rangle_{r}$ as estimated from the inset shown in (a) is indicated as dash-dotted, black line. The theoretical value of $25 \mu \mathrm{m} \mathrm{W}$ is indicated as dashed, black line. (c) shows a close-up of (b). Here it can be seen that $\gamma=2.496 \times 10^{3}$ is in good agreement with the reference. 
Finally, a quantification of the achieved resolution on the reconstructed phase maps of the reference dataset 2 and the STCS dataset 1, where the scaling factor $\gamma=2.496 \times 10^{3}$ yields the best agreement with the reference, is presented. Reconstructed phase maps corresponding to dataset 1 and 2, which are already shown in Fig. 3.60 are presented in Figs. 3.62 (a) and (b), respectively. The reconstructed probe fields are presented in Figs. 3.63 (a) and (b). The power spectral densities (PSD) of both reconstructed phase maps were analysed. The 2D PSDs were calculated by using a Kaiser-window (cf. [95, 330 and section 1.1.2, p. 12) and are presented in Figs. 3.62 (c), (d). In order to better compare the frequency content of the reconstructions, the 2D PSDs have been azimuthally averaged and are depicted in Fig. 3.62 (e).

Although the PSD gives a proper measure of the frequency content of the reconstructed phase map the resolution achieved by ptychographic phasing needs to be carefully justified. For this reason the phase retrieval transfer function (PRTF), which compares the measured amplitudes $I^{\text {meas }}$ to the reconstructed ones $I^{\mathrm{P}}$ (cf. 97, 274, 274 and equation 3.111, p. 82), was also calculated. The PRTFs of both datasets are shown in Fig. 3.62 (f).

\subsubsection{Results \& Discussion}

A comparison of line profiles through the centre of diffraction patterns from dataset 1 and 2 (Figs. 3.59 (f), (g)) shows a good agreement of the rescaling in the central region of the diffraction pattern of dataset 1 with the one corresponding to dataset 2 .

Next, the influence of different scaling factors $\gamma=1 /\left(I / I_{0}\right)=\exp (\mu d)$ for the STCS region of dataset 1 (cf. Fig. 3.60) was analysed. By comparison to the reconstruction obtained without the STCS (dataset 2) one can read off an optimum scaling factor of $\sim 2.496 \times 10^{3}$, which deviates by a factor of 0.62 from the theoretical value. The comparably large relative uncertainty $\Delta \gamma / \gamma=\left|\gamma-\gamma_{\text {theo }}\right| / \gamma=0.62$ can be explained by the Beer-Lambert law. Applying the Beer-Lambert law $I=I_{0} \exp (-\mu d)$ yields the dependence of the relative uncertainty of the thickness of the used STCS foil $\Delta d / d$ on the relative uncertainty of the scaling factor:

$$
\begin{aligned}
\Delta I & =-\mu I \Delta d=-\log (\exp (\mu d)) I \Delta d / d \\
\Rightarrow \Delta \gamma & =-\frac{I_{0}}{I^{2}} \Delta I=\log (\gamma) \gamma \Delta d / d
\end{aligned}
$$

which yields the final result

$$
\Delta \gamma / \gamma=\log (\gamma) \Delta d / d
$$

The explicit occurrence of the scaling factor implies an accurate knowledge of the STCS thickness. For instance, using $\Delta d / d=0.1$ and $\gamma=4.054 \times 10^{3}$, which correspond to the experimental parameters, yields $\Delta \gamma / \gamma=0.83$, which is in good agreement with the experimental result.

For the optimum scaling factor $\gamma \approx 2.496 \times 10^{3}$ the reconstructed phase shift of $<\Delta \Phi>=-0.755 \mathrm{rad}$ (Ta vs. no Ta) deviates less than $1 \%$ from the reference 
(a)
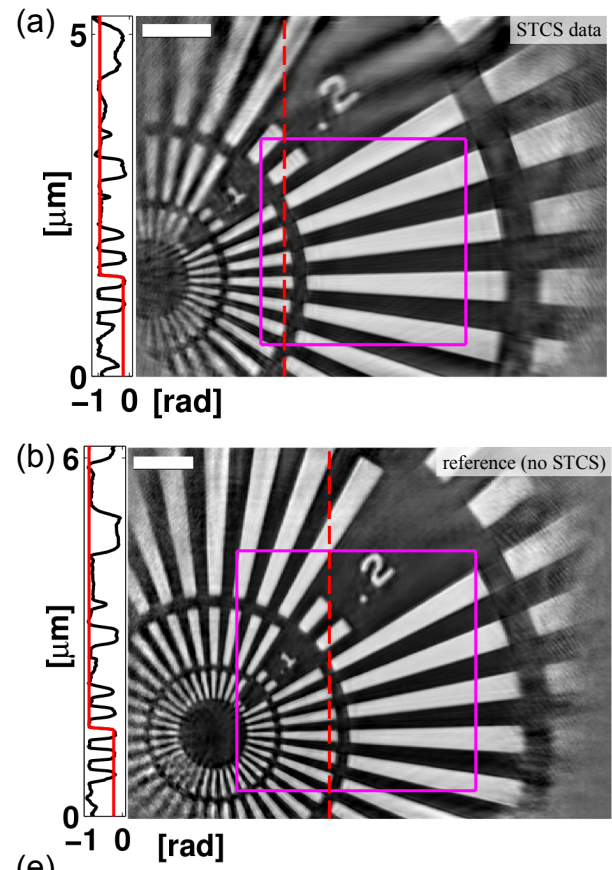

(e)

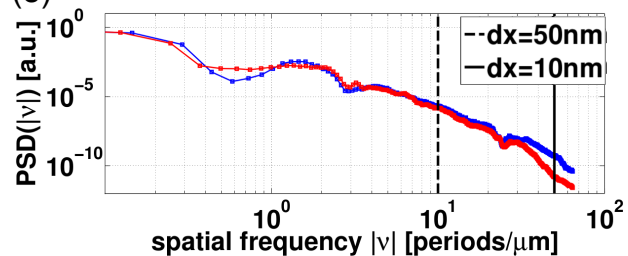

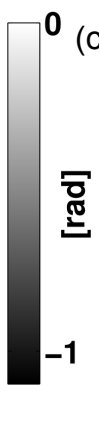
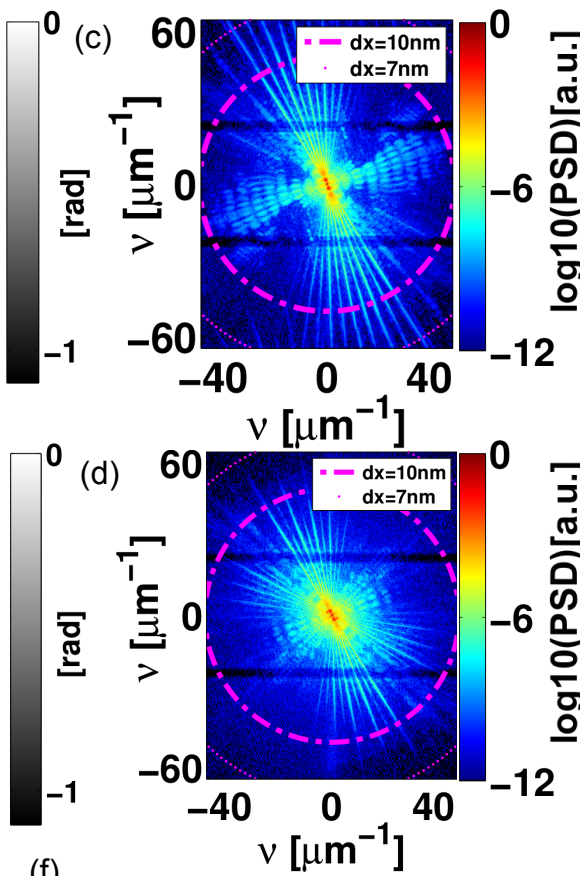

(d)

(f)

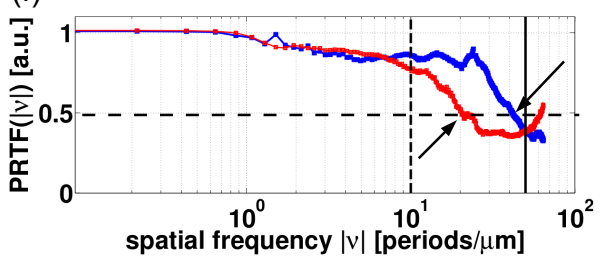

Figure 3.62: (a), (b) present phase reconstructions of the resolution chart sample as obtained from data taken with a photon flux of $\sim 2 \times 10^{8}$ phot/s and STCS and with a photon flux of $\sim 4 \times 10^{6} \mathrm{phot} / \mathrm{s}$ and no STCS, dataset 1 and 2 respectively. In both cases the sample was scanned over a rectangular area as indicated by magenta lines. Fits of the error function to the edges of the line scans (dashed, red lines) yields line-spread functions with FWHM-values of $23 \mathrm{~nm}$ and $26 \mathrm{~nm}$, in (a) and (b) respectively. (c) and (d) show the calculated 2D power-spectral densities (PSD) of the reconstructed phases depicted in (a) and (b), respectively. Frequencies corresponding to a real space pixel size of $10 \mathrm{~nm}$ and $7 \mathrm{~nm}$ are indicated as magenta circles. (e) and (f) show PSDs and PRTFs from both datasets after azimuthal averaging. Dataset 1 is represented in blue whereas dataset 2 is represented in red. The arrows in (f) indicate the PRTF cut-off at about 0.5 yielding a half-period resolution of $12 \mathrm{~nm}$ and $25 \mathrm{~nm}$ for dataset 1 and 2, respectively. Frequencies corresponding to real space pixel sizes of $50 \mathrm{~nm}$ and $10 \mathrm{~nm}$ are further emphasized in (e) and (f) by vertical lines. Scale bars denote $1 \mu \mathrm{m}$ in (a) and (b). 

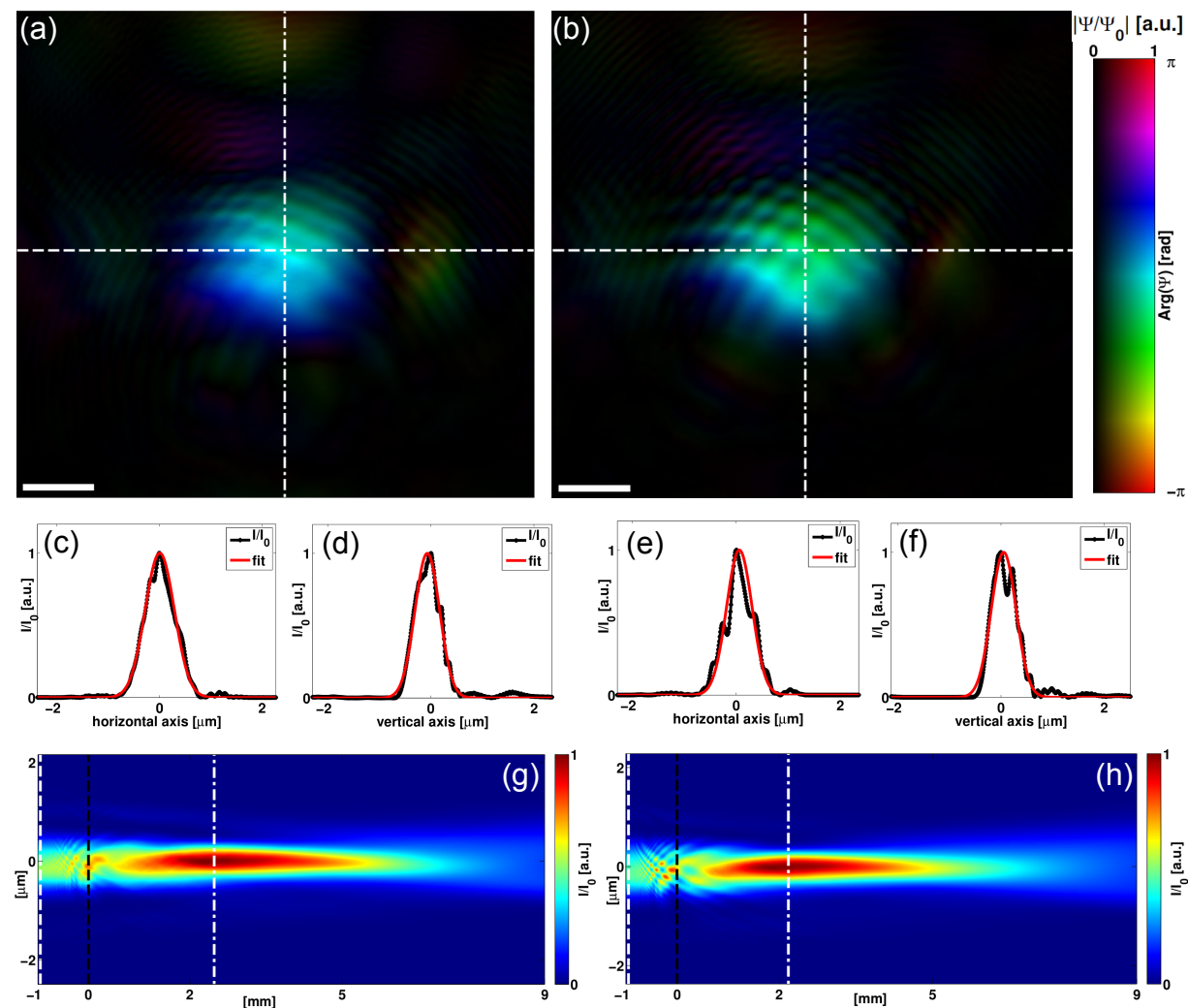

Figure 3.63: (a), (b) visualisations of the central part of the reconstructed complex probe fields of dataset 1 and dataset 2, respectively. (c) intensity of horizontal slice through probe at the sample (dashed, white line in (a)). (d) intensity of vertical slice through probe at the sample (dash-dotted, white line in (a)). Gaussian fits (red lines) yield FWHM values of $673 \mathrm{~nm} \times 564 \mathrm{~nm}(\mathrm{~h} \times \mathrm{v})$. (e) intensity of horizontal slice through probe at the sample (dashed, white line in (b)). (f) intensity of vertical slice through probe at the sample (dash-dotted, white line in (b)). Gaussian fits (red lines) yield FWHM values of $580 \mathrm{~nm} \times 566 \mathrm{~nm}(\mathrm{~h} \times \mathrm{v})$. $(\mathbf{g}),(\mathbf{h})$ intensity of numerically propagated probe fields as slice through the optical axis and the horizontal axis (indicated as dashed, white line in (a),(b)) of the focus (at $z \gtrsim 2 \mathrm{~mm}$ ) of dataset 1 and 2, respectively. Dashed white lines, dashed black lines and dash-dotted white lines indicate plane of the pinhole, position of the sample and position of the focal plane, respectively. Scale bars denote $0.5 \mu \mathrm{m}$ in (a) and (b). 
value (cf. Tab. 3.11). Interestingly, a lower than optimum scaling factor results in an increased phase contrast of the object. The tolerance for the reconstruction is surprisingly large, spanning roughly one order of magnitude between the lowest tolerable value and the optimum. Towards higher values than optimum, the tolerance is even larger: although loosing more and more phase contrast the object can still be clearly identified at an over-scaling of 3 orders of magnitude. The reason for this result may be explained as follows: assuming that the STCS region contains only low frequency information about the probe, the intensity scaling acts as a filter that shifts the relative contributions between object and probe. If the scaling is too low (in the limit of becoming a fully absorbing beam stop) the information about the probe is highly suppressed in the data causing a violation of the probe assumptions in the algorithm. With an increasing scaling factor $\gamma$, the relative weight of frequency contributions from the object is decreased.

The visual impression of the high resolution phase maps (Figs. 3.62 (a), (b)) is very good. The innermost Siemens star ring structure ending at a width of $50 \mathrm{~nm}$ can be clearly resolved by both datasets. In the case of dataset 1 this is already a hint for the high resolution obtained as the innermost structure does not fall into the scanned area marked by a magenta rectangle. The part outside this area is only illuminated by the tails of the illuminating wave-field and is thus illuminated by considerably less photons. The resolution can be further analysed by fitting an error function to one of the edges occurring in the image. Line-spread functions with a full width at half maximum (FWHM) of $23 \mathrm{~nm}$ and $26 \mathrm{~nm}$ at singular positions in dataset 1 and dataset 2 (Figs. 3.62 (a), (b)) were obtained, respectively. The calculated 2D PSDs yield a resolution estimation of the whole phase maps (Figs. 3.62 (c), (d)). Note that there are two horizontal stripes in the 2D PSDs which contain less information about the sample due to the insensitive regions of the detector between the modules. Besides, one can clearly see the frequency contributions arising from the star form of the object. There are frequency contributions corresponding to pixel sizes in the range from $10 \mathrm{~nm}$ down to $7 \mathrm{~nm}$ for dataset 1 , whereas the frequency content of dataset 2 seems to end at $10 \mathrm{~nm}$. The azimuthal average of the PSDs as shown in the subfigure (e) confirms this result. In the high frequency part of the spectrum the curve of dataset 1 (blue curve) lies well above the one of dataset 2 (red curve). The difference in resolution is seen more drastically by comparing the PRTFs (Fig. 3.62 (f)). Now the resolution is judged by the frequency where the PRTF drops below the cut-off of 0.5. A half-period cut-off corresponding to a pixel size of $12 \mathrm{~nm}$ and $25 \mathrm{~nm}$ for dataset 1 and 2 is obtained, respectively. Here, the cut-off likely yields an upper limit and the actual resolution is better.

Next, the question how good can the probe of the rescaled STCS data be recovered is addressed. The probes, as reconstructed in the plane of the sample, are thus presented in Figs. 3.63 (a) and (b). It is found that the probe reconstruction from the STCS data is in good agreement with the probe reconstruction without STCS (reference). Both probes exhibit a similar shape and fringe pattern which arises from a non-optimum alignment of the pinhole. The small differences in the fringe pattern are likely due to a drift of the pinhole during the time delay between both 
dataset 1 and 2 have been recorded. The reliability of the reconstruction is further supported by the similarity of the propagated probe fields along the optical axis (Figs. $3.63(\mathrm{~g}),(\mathrm{h})$ ). Here it is pointed out again that the comparably large probe size with FWHM values of $673 \mathrm{~nm} \times 564 \mathrm{~nm}(\mathrm{~h} \times \mathrm{v}$; dataset 1 ; Figs. 3.63 (c), (d) $)$ and $580 \mathrm{~nm} \times 566 \mathrm{~nm}(\mathrm{~h} \times \mathrm{v}$; dataset 2; Figs. $3.63(\mathrm{e})$, (f) $)$ arises from the particularly small slit setting, which has been chosen to match the beam size on the detector to the size of the STCS. The quality of the obtained reconstructions shows that this beam size does not represent a limitation. On the contrary, the high resolution and relatively large beam are an ideal combination in view of imaging a large field of view and tomography.

\subsubsection{Summary \& Conclusion}

In summary, a study on the use of Semi-Transparent Central Stops (STCS) for hard X-ray ptychographic imaging was perfomed. By using the STCS, the central beam on the detector is attenuated by ca. four orders of magnitude and thereby the usable photon flux for ptychography could be increased by two orders of magnitude. The fact that the usable flux could not be increased further e.g. by further reduction of the global attenuation factor was due to the specific nature of the test pattern. It turns out that low frequency components contribute to a high scattering intensity very close to the central beam (cf. Figs. 3.59 (c), (d)). Hence, the efficiency of the STCS also depends on the object being imaged. For instance, a biological specimen with uniform scattering distribution may be studied with a lower global beam attenuation.

The STCS data has been compared with reference data taken without the STCS. It was found that the phase deviation for optimum scaling factors between both reconstructions is less than $1 \%$. Here, the point may be stressed that usual error specifications for tungsten foils of $25 \mu \mathrm{m}$ thickness are in the $10 \%$ range, which already has a great impact on the actual transmission. Hence, one may be tempted to invest more into the quality of the material used for STCS application. This might not be necessary for all applications because a relaxation of the requirement on quantitativeness of the phase of the object relaxes the necessary absolute value used for rescaling of the attenuated region on the detector.

It was found that the reconstruction process is quite robust when changing the scaling of the STCS region by multiple orders of magnitude. In addition, the increase in resolution was determined to be up to a factor of two, depending on the criterion used, when using the STCS. The resolution achieved is $12 \mathrm{~nm}$ over large parts of the sample and may be as good as $7 \mathrm{~nm}$ in particular regions.

The STCS technique at the Göttingen Instrument for Nano-Imaging with X-rays (GINIX) can still be improved, as the optimum fully available coherent photon flux of $\sim 10^{11} \mathrm{phot} / \mathrm{s}$ is still almost three orders of magnitude away from the current experimental setting. In future, the feasibility of higher attenuation factors as well as an STCS with compound attenuation values, i.e. a more complex attenuation profile, will thus be studied. One possible design that may be particularly suited for high resolution ptychographic imaging of the Siemens star pattern using the 
same experimental parameters is presented in Fig. 3.64 (see also section G. p. 197). Here, the concept of using two different attenuation strengths is realised: The direct beam is highly attenuated whereas the low q-diffraction contributions are gently attenuated. The prototype in Fig. 3.64 was fabricated from two pieces of Si wafer. Notably, the thickness description for commercially available Si wafer products is likely very accurate which may facilitate the determination of the rescaling factor. However, the practicability of such compound attenuation profiles has not been tested yet.

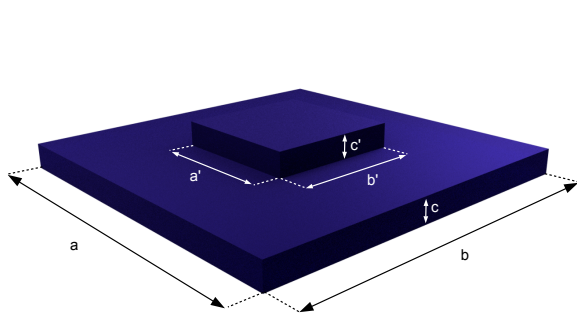

(a)

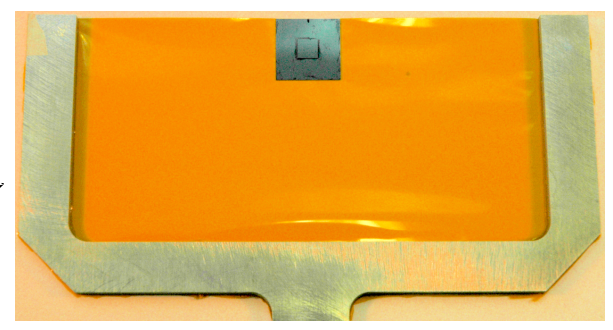

(b)

Figure 3.64: The figure illustrates the design of an STCS with two attenuation regions. (a) indicates dimensions. (b) presents a prototype made of two pieces of Si wafer (thickness $c=c^{\prime}=200 \mu \mathrm{m}$ ) with lateral dimensions $a=b=16 \mathrm{~mm}$ and $a^{\prime}=b^{\prime}=$ $5 \mathrm{~mm}$. The theoretical transmission values for $200 \mu \mathrm{m}$ and $400 \mu \mathrm{m}$ Si are $5.04 \cdot 10^{-2}$ and $2.54 \cdot 10^{-3}$ at $7.9 \mathrm{keV}$ photon energy $\left(\rho_{\mathrm{Si}}=2.363 \mathrm{~g} / \mathrm{cm}^{3}\right) \mid 125$, respectively. Note that the thickness of $400 \mu \mathrm{m}$ in the centre almost perfectly compensates the overall attenuation of beam for the case of dataset 2 where $444 \mu \mathrm{m}$ Si attenuation was used. The cutting of the wafer pieces was carried out using a dicing saw Disco dad 321 (Disco, Japan; cf. section 3.4 p. 144).

An important future application may arise from ptychographic imaging of biological specimens or other weakly scattering objects. The STCS application is not restricted to ptychographic imaging. In Coherent Diffractive Imaging (CDI) applications the central beam is typically fully blocked by a suitably dimensioned central stop and the desired image can still be reconstructed. The introduction of an STCS in CDI may relax the demands on choosing the right dimensions while simultaneously providing access to the low q-information about the direct beam without any change of the set-up. For instance, a slight change of the beam centre could be observed while taking data. Furthermore, the STCS will find useful application in classical diffraction analysis, where one also looses low q-information through the use of a fully absorbing beam stop. 


\subsection{High Flux Ptychographic Imaging Using the New $55 \mu \mathrm{m}-$ Pixel Detector 'Lambda' Based on the Medipix3 Read- out Chip: an STCS Application}

Suitable detection systems that are capable to record high photon count rates with single photon detection are instrumental for coherent X-ray imaging. Here, the new single photon counting pixel detector 'Lambda' was tested in a ptychographic imaging experiment on solar cell nanowires using KB-focused $13.8 \mathrm{keV}$ X-rays. Taking advantage of the high count rate of the Lambda and dynamic range expansion by the semi-transparent central stop, a high dynamic range diffraction signal covering more than 7 orders of magnitude has been recorded which corresponds to a photon flux density of about $10^{5} \mathrm{phot} / \mathrm{nm}^{2} / \mathrm{s}$ or a flux of $\sim 10^{10} \mathrm{phot} / \mathrm{s}$ on the sample. By a comparison with data taken without the semi-transparent central stop, an increase in resolution by a factor $3-4$ is determined: from about $125 \mathrm{~nm}$ to about $38 \mathrm{~nm}$ for the nanowire and from about $83 \mathrm{~nm}$ to about $21 \mathrm{~nm}$ for the illuminating wave-field.

Coherent Diffractive Imaging $\mid 41,197,297]$ and, in particular, its variant ptychography $81,249,296$ needs to cope with diffration patterns spanning orders of magnitude which can be problematic for current photon counting detectors. In case of ptychography, this high dynamic range problem is more accentuated because both the strong signal from the primary beam and the weak diffracted signal from the probed object need to be recorded. Intended as high resolution X-ray imaging techniques, the potential resolution is considered to be limited by the invested dose 141. Consequently, a high primary beam intensity is needed to achieve a high resolution. Numerical phasing of the diffraction data thus crucially depends on modern detectors which provide a high dynamic range. An alleviation of the high dynamic range problem can be achieved by giving up strict photon counting. For instance, the MM-PAD 289,311 achieved a count rate of $10^{8} \mathrm{phot} / \mathrm{pixel} / \mathrm{s}$ at $8 \mathrm{keV} \mathrm{X}$-rays in a recent ptychography study [96]. Another important development in this direction is the Adaptive Gain Integrating Pixel Detector 236. In addition to the high dynamic range, fast readout, small pixels and low noise are also important. Single photon counting pixel detectors such as Pilatus [163] and Maxipix 176 have the advantage of effectively zero readout noise and can reach maximum count rates in the range of $10^{5}-10^{7} \mathrm{phot} / \mathrm{pixel} / \mathrm{s}$ 306 308]. The new Large Area Medipix-Based Detector Array (Lambda) is being developed 225 227 in order to better meet experimental demands. Based on the Medipix3 chip |11], the Lambda is constructed with two counters for deadtime-free readout. In the standard mode it can operate at 2000 frames/s. In combination with its small pixel size and the increased maximum photon count rate of about $3 \cdot 10^{5} \mathrm{phot} / \mathrm{s} / \mathrm{pixel}[10$ in comparison to its predecessor with the Medipix 2 chip, the Lambda becomes thus a valuable device in synchrotron applications such as ptychography. Another important detector development, albeit with a larger pixel area (by a factor of 1.86), for single-photon counting while framing at $\mathrm{kHz}$ rates is the $75 \mu \mathrm{m}$-pixel detector 'Eiger' 63, 149 which, very recently, was successfully operated in a ptychographic imaging experiment 112 . 
Nevertheless, achieving an optimum resolution in ptychographic imaging remains a challenge. On the algorithmic side, the results can be improved by e.g. correcting

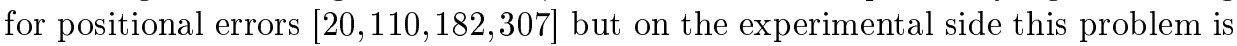
currently mainly approached by increasing the fluence, e.g. \begin{tabular}{|l|l|l|l|}
111 & 133 & 266 & 286 \\
287
\end{tabular} 332 .

In this section, the performance of the Lambda detector in a ptychographic imaging experiment on solar cell nanowires using $13.8 \mathrm{keV}$ photons at the high brilliance synchrotron radiation source PETRAIII is investigated. In order to make use of the full, highly coherent flux of the KB-focus the Lambda is tested in combination with the Semi-Transparent Central Stop (STCS) 332 . The results within this section can also be considered as a further application to preceding STCS experiments (cf. section 3.3, p. 132) but here a step towards real applications was made with the nanowire sample. In addition, the ptychographic probe reconstruction is used to monitor the systematic variation of the beam size due to a change of the numerical aperture in front of the Kirkpatrick-Baez (KB) mirrors. The fluence on the sample can be accurately determined which in turn is used to achieve a fluence optimised setting. The section closes with a short discussion of the results in view of the Lambda detector.

This section is based on results that have been published in 333 . J. Wallentin's contributions of the nanowire sample and corresponding SEM images are gratefully acknowledged.

\subsubsection{Experimental Set-up}

The experiments with the 'Lambda' detector were performed at the P10 coherence beamline of the PETRA III synchrotron located at DESY, Hamburg in Germany. A monochromatic X-ray beam with an energy of $13.8 \mathrm{keV}$ was selected by using a channel-cut monochromator $(\mathrm{Si}(111))$ [343. After passing a set of beam defining slits, the beam was focused by a pair of X-ray mirrors in the Kirkpatrick-Baez geometry (cf. Fig. 3.65 (a)). The elliptically shaped mirrors (Pd coating) were used at $4.05 \mathrm{mrad}$ inc. angle (centre). The focal distances are $302 \mathrm{~mm}$ and $200 \mathrm{~mm}$ of the vertical mirror (WinlightX) and horizontal mirror (JTEC), respectively (for details see $[152-154)$. A soft-edge, rectangular aperture was placed $12 \mathrm{~mm}$ upstream of the nominal focal plane to suppress side-lobes of the X-ray beam [286]. The scanning of the sample through the X-ray beam was carried out by a highprecision piezo-electric stage (PI, Germany). Both the KB X-ray mirror system and the used sample positioning stages are part of the Göttingen Instrument for Nano-Imaging with X-rays (GINIX). An evacuated flight-tube of $5 \mathrm{~m}$ length was installed behind the sample stage to minimize scattering effects of X-rays in air. A positionable semi-transparent central stop was installed in the flight-tube. The Lambda detector was placed a few centimetres behind the exit window of the flight-tube. The distance between focal plane and Lambda was measured to be $5.07 \mathrm{~m}$. 

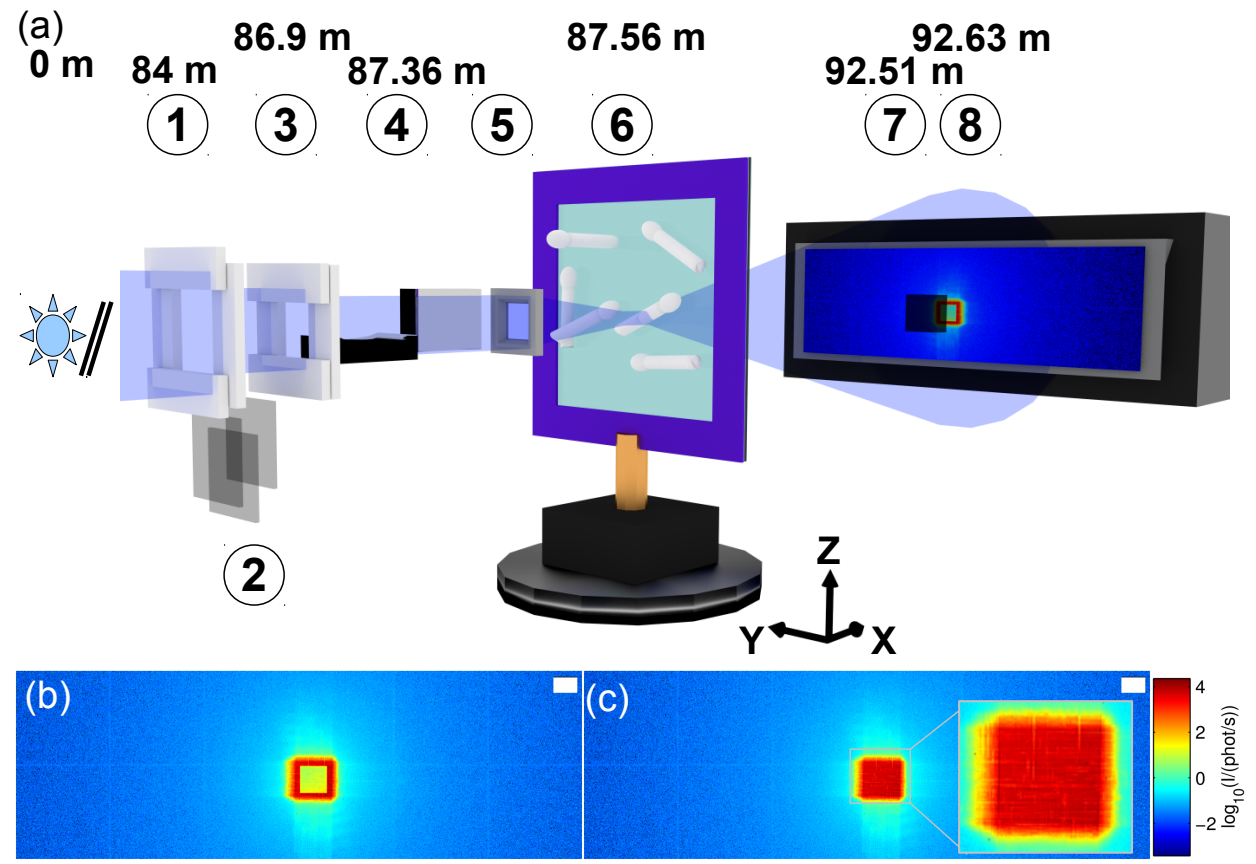

Figure 3.65: (a) Schematic of the GINIX set-up: downstream of the undulator source and the monochromator (not shown) (1) slits S1, (2) attenuation foils made of Mo, (3) slits S2, (4) KB-mirror system (position of the horizontal mirror ), (5) soft-edge aperture ( $\sim 12 \mathrm{~mm}$ in front of the focus), (6) nanowire sample in the focal plane, $(7)$ STCS (Ge) and (8) Lambda detector. The flight-tube between sample and detection device is not shown in the graph. (b) shows intensity measurement recorded with the Lambda and the STCS (7). (c) Same intensity pattern as in (b) but rescaled according to measured STCS-attenuation distribution. Scale bars in (b) and (c) denote $q=50 \mu \mathrm{m}^{-1}$. Note that scaling of (b), (c) differs from presentation in 333 . 


\subsubsection{Details: STCS Preparations}

The STCS is a piece of germanium with lateral dimensions of about $4 \mathrm{~mm} \times 4 \mathrm{~mm}$, which was cut from a wafer using a dicing saw Disco dad 321 (Disco, Japan). Additionaly, the Ge STCS was grinded down to an average nominal thickness of $100 \mu \mathrm{m}$ using $\mathrm{SiC}$ grinding paper with successive grain sizes 2000 and 4000 on a grinding machine LaboPol-21 (Struers).

The attenuation profile of the STCS was measured with the Lambda by using an open slit setting of $\mathrm{S} 1=\mathrm{S} 2=300 \mu \mathrm{m} \times 300 \mu \mathrm{m}$ and sufficient beam attenuation (Fig. 3.65(b)). The rescaling of STCS-attenuated diffraction patterns is exemplified in figure 3.65 (c) whereas the measured thickness distribution (attenuation profile) and a photo of the STCS are presented in figure 3.66 .

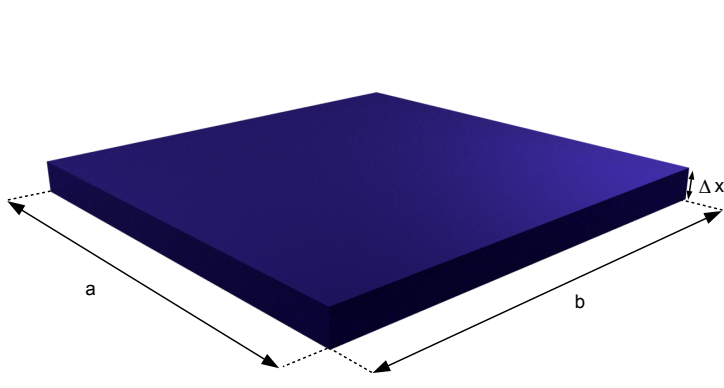

(a)

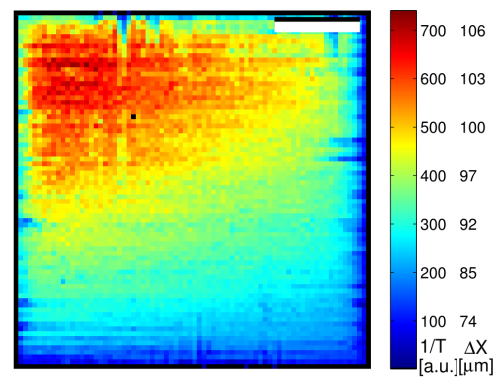

(b)

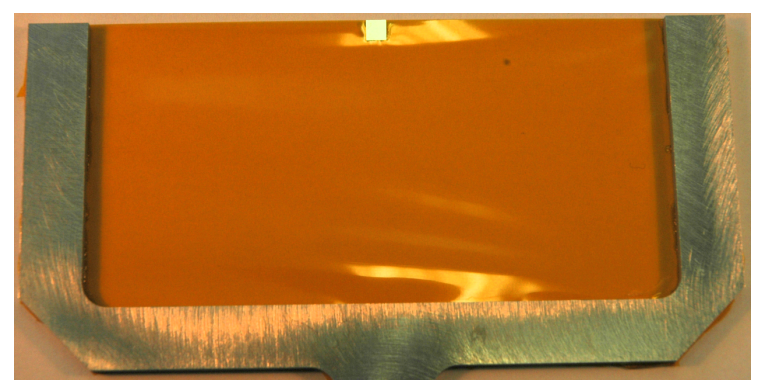

(c)

Figure 3.66: (a) illustrates dimensions of the Ge STCS ( $a=b \cong 4 \mathrm{~mm}, \Delta x \approx 100 \mu \mathrm{m})$. (b) STCS thickness $\Delta X$ determined from measured transmission $T$ and tabulated linear absorption coefficient $\left(\mathrm{Ge}, \rho=5.323 \mathrm{~g} / \mathrm{cm}^{3}\right)[125]$. (c) shows the a photo of the holder of the Ge STCS. Here, the STCS is glued on top of $25 \mu \mathrm{m}$ polymide foil (Kapton). The scale bar in (b) denotes $1 \mathrm{~mm}$.

\subsubsection{Samples}

The nanowires in this work (Fig. 3.67) were grown and processed as described in 319,320 . Briefly, (i) gold seed particles were formed on an InP substrate by 
nanoimprint lithography 179 , (ii) InP nanowires were grown with vapor phase epitaxy, (iii) the gold was removed by wet etching, (iv) $\mathrm{SiO}_{2}$ deposition (insulator) by atomic layer deposition and ( $\mathrm{v}$ ) sputter deposition of an optically transparent and conducting layer of indium tin oxide (ITO). After fabrication, the nanowires were cut off the substrate by gently wiping the surface with a soft tissue. Nanowires on the tissue were then allowed to adhere on a $\mathrm{Si}_{3} \mathrm{~N}_{4}$ membrane ( $1 \mu \mathrm{m}$ thickness) (Silson, UK). This process yields randomly distributed nanowires (Fig. 3.67 (b)), which occasionally are oriented perpendicular to the membrane (Fig. 3.67 (c)). After the X-ray experiment the sample was sputtered with a $5 \mathrm{~nm}$ gold layer for SEM imaging.
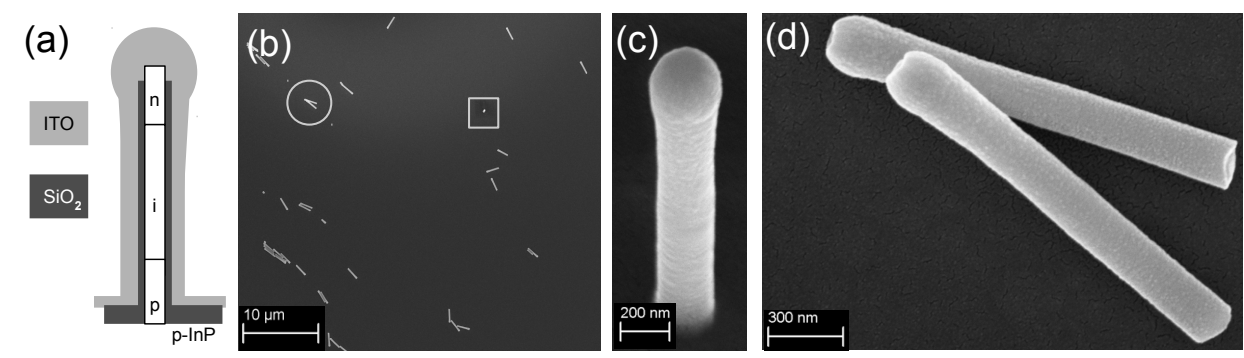

Figure 3.67: (a) schematic of a nanowire solar cell consisting of InP substrate (p-type at the bottom and n-type on top), an insulating $\mathrm{SiO}_{2}$ layer and an ITO cover. (b) shows a SEM image of the nanowires deposited on the $\mathrm{Si}_{3} \mathrm{~N}_{4}$ membrane after the X-ray experiment. (c) is a SEM image of the standing nanowire in (b) which is highlighted by a grey rectangle. The view is at an angle of $45^{\circ}$. (d) shows two lying nanowires of the sample (cf. circle in (b)). Here, the diameter was measured to be about $300 \mathrm{~nm}$ at the body and $360 \mathrm{~nm}$ at the head. The length is about $2.6 \mu \mathrm{m}$.

\subsubsection{Details: Data Recording \& Data Treatment}

The first ptychographic scan (dataset 1) covers an area of $3 \mu \mathrm{m} \times 3 \mu \mathrm{m}$ around the nanowire that was oriented perpendicular to the membrane or 'standing' (Fig. 3.67 (c)). Here, $31 \times 31$ scan points were taken on a rectangular grid with step sizes of $100 \mathrm{~nm}$. At each point the exposure time was $1 \mathrm{~s}$. Including movements of motors and readout, the total scanning time was about $29 \mathrm{~min}$. The scan of dataset 2 was recorded on a nanowire being oriented parallel to the membrane ('lying') with same parameters as dataset 1 , except only $26 \times 26$ scan points were distributed equidistantly over a square of $2.5 \mu \mathrm{m} \times 2.5 \mu \mathrm{m}$. For both datasets a small beam defining slit gap S1 of $50 \mu \mathrm{m}$ was chosen and the photon flux impinging on the sample was reduced by molybdenum foils to about $10^{7}$ phot/s (cf. Tab. 3.12). The ptychographic dataset 3 was taken on the same nanowire with identical scan parameters as dataset 2 . The total scanning time was about $20 \mathrm{~min}$ in both cases. In contrast, the beam defining slit gap S1 was opened to $100 \mu \mathrm{m}$, which corresponds to an increase in flux by a factor of about 4 . Importantly, no beam attenuation in front of the sample was used and the higher photon flux (increase by a factor 
of $\left.\sim 10^{3} / 5.3\right)$ of the KB beam on the detector was compensated by inserting the STCS in front of the Lambda detector.

Table 3.12: The table summarizes information on used slit settings.

\begin{tabular}{lllll}
\hline \multirow{2}{*}{ Dataset } & slits & $\begin{array}{l}\text { Horizontal } \\
\text { gap }[\mu \mathrm{m}]\end{array}$ & $\begin{array}{l}\text { Vertical } \\
\text { gap }[\mu \mathrm{m}]\end{array}$ & $\begin{array}{l}\text { Photon } \\
\text { flux }[\mathrm{phot} / \mathrm{s}]\end{array}$ \\
\hline \multirow{2}{*}{1} & $\mathrm{~S} 1$ & 50 & 50 & $1.06 \cdot 10^{7}$ \\
& $\mathrm{~S} 2$ & 200 & 200 & \\
2 & $\mathrm{~S} 1$ & 50 & 50 & $1.10 \cdot 10^{7}$ \\
& $\mathrm{~S} 2$ & 200 & 200 & \\
3 & $\mathrm{~S} 1$ & 100 & 100 & $9.8 \cdot 10^{9}$ \\
& $\mathrm{~S} 2$ & 200 & 200 & \\
\hline
\end{tabular}

The design of the Lambda detector here consists of 12 Medipix 3 chips which at the corners consist of $255 \times 255$ pixels. In between they are of a size of $254 \times 255$ pixels. The gaps between the chips are filled with pixels larger than the chip pixels $(55 \mu \mathrm{m} \times 55 \mu \mathrm{m})$. There are $2 \times 2$ pixels in the intersections of horizontal and vertical gaps with a size of $165 \mu \mathrm{m} \times 165 \mu \mathrm{m}$. The remaining pixels in the gaps are $55 \mu \mathrm{m} \times 165 \mu \mathrm{m}(\mathrm{h} \times \mathrm{v})$ and $165 \mu \mathrm{m} \times 55 \mu \mathrm{m}(\mathrm{h} \times \mathrm{v})$ in the horizontal and the vertical gaps, respectively. The data in the gap pixels were rebinned to the size of the chip pixels.

The ptychographic reconstruction for both object and probe function was carried out using the 'ePIE' algorithm (using $\alpha=\beta=0.5$; cf. [183]). The algorithmic settings are the same for all three datasets. The reconstruction procedure was initialised using a Gaussian (FWHM $=200 \mathrm{~nm}$ ) amplitude distribution as probe guess and an unity, pure amplitude plane as object. The number of ptychographic iterations was 600 . The final results of the probe and object were obtained by averaging the current estimate over the last 200 iterations.

\subsubsection{Results: Ptychographic Reconstruction of KB Wave-Fields}

The results of the ptychographically reconstructed complex wave-field incident on the sample of dataset 1 (S1 gap $50 \mu \mathrm{m})$ are presented in figure 3.68. In a first step, the reconstructed probe was numerically propagated along the optical axis by $\pm 8 \mathrm{~mm}$. The focal plane was determined to be $240 \mu \mathrm{m}$ upstream of the sample by using the 'sharpness' criterion (e.g. $[108$ and see equation 3.115, p. 84).

The complex wave-field of the focal plane is shown in figure 3.68 (a). It can be seen that side lobes are more pronounced in the vertical direction. The phase representation in the image reveals an effectively flat phase of the central peak. Line cuts through the maximum of the peak can be seen in figures 3.68 (b), (c). Here, the difference in side lobe formation between the horizontal and vertical 
(a)

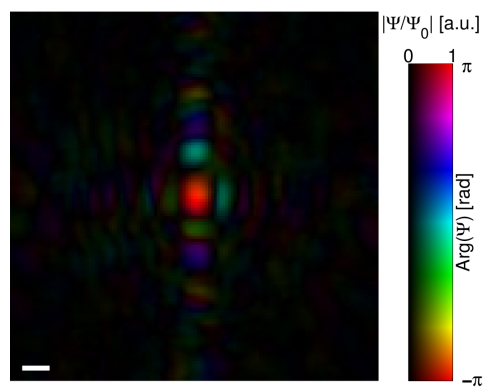

(c)
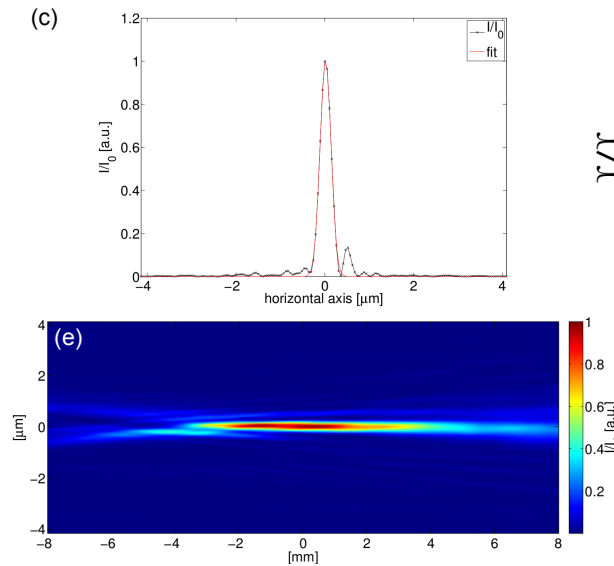

(b)

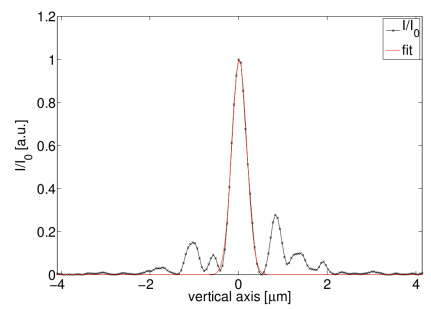

(d)
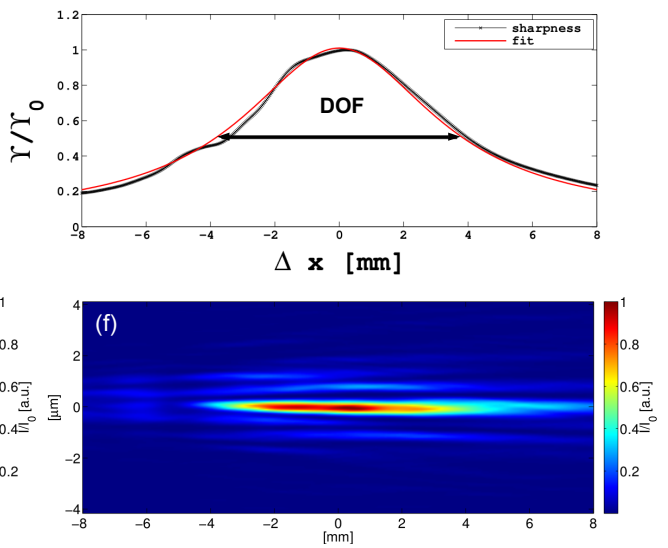

Figure 3.68: (a) complex field of the probe of the low photon flux dataset 1 (cf. Tab. 3.12 and Fig. 3.70) propagated to the focal plane. Amplitude and phase are drawn according to the colourbar next to the image. In (b) and (c) line-cuts through the vertical and horizontal direction of the probe intensity in the focus (a) are drawn, respectively. Gaussian fits to the curves (red lines) yield FWHM $=393 \mathrm{~nm}$ and FWHM $=292 \mathrm{~nm}$ in (b) and (c), respectively. (d) sharpness and fit for estimation of the DOF. (e) shows the horizontal slice of the probe intensity through the focus. (f) presents vertical slice. Scale bar in (a) denotes $500 \mathrm{~nm}$. 
direction is visible, too. Using a Gaussian fit to determine the size of the focus yields $\mathrm{FWHM}=292 \mathrm{~nm}$ in the horizontal direction. For the vertical direction FWHM $=393 \mathrm{~nm}$ is obtained. A horizontal and a vertical slice through the focus and along the optical axis is shown in figures 3.68 (e) and (f), respectively. The depth of focus (DOF) extends visibly over a few millimetres. The 'sharpness' can also be used to quantify the DOF. In case of a Gaussian intensity distribution $I(\rho, x)=\left(I_{0} /\left(1+\left(x / x_{0}\right)^{2}\right)\right) \exp \left(-2 \rho^{2} /\left(w_{0}^{2}\left(1+\left(x / x_{0}\right)^{2}\right)\right)\right)$ along the radial direction $\rho=\sqrt{y^{2}+z^{2}}$ with Rayleigh length $x_{0}$ and beam waist $w_{0}$, the sharpness can be analytically expressed:

$$
\Upsilon_{G}(x)=(\pi / 4)\left(I_{0} w_{0}\right)^{2} /\left(1+\left(x / x_{0}\right)^{2}\right) .
$$

Therefore, the normalized sharpness $\Upsilon_{G}(x) / \Upsilon_{G}(0)$ is a measure of the Rayleigh length $x_{0}$ of the beam, which is equal to half of the DOF. A fit of $\Upsilon_{G}(x)$ to the sharpness of the reconstructed probe field yields DOF $=2 x_{0}=7.32 \mathrm{~mm}$ (cf. Fig. $3.68(\mathrm{~d}))$.

The reconstructed wave-field of dataset $3(\mathrm{~S} 1$ gap $100 \mu \mathrm{m})$ is analysed in the same way. In this case, the focal plane almost coincides with the plane of the sample. The distance between sample plane and focal plane is determined to be $16 \mu \mathrm{m}$. The intensity distribution of the focal plane reveals a smaller peak with reduced side lobes (cf. Fig. 3.69 (a)). In the line cuts through the central peak it can be seen that the intensity of the side lobes are reduced by a factor of about 2 in comparison to the other probe field. Gaussian fits to the curves yield a beam width of FWHM $=217 \mathrm{~nm}$ and FWHM $=136 \mathrm{~nm}$ in the vertical (Fig. 3.69 (b)) and horizontal (Fig. 3.69 (c)) direction, respectively. Hence, the decrease in size is by a factor of $1 / 2.15$ in the horizontal direction and by a factor of $1 / 1.81$ in the vertical direction. Moreover, the decrease in size of the beam can also be observed in the slices through the focus and along the optical axis as a decrease in the depth of focus (cf. Fig. 3.69 (e), (f)). A fit of $\Upsilon_{G}(x)$ to the sharpness of the reconstructed probe field yields $\mathrm{DOF}=2 x_{0}=1.52 \mathrm{~mm}$ (cf. Fig. 3.69 (d)). 
(a)

(c)
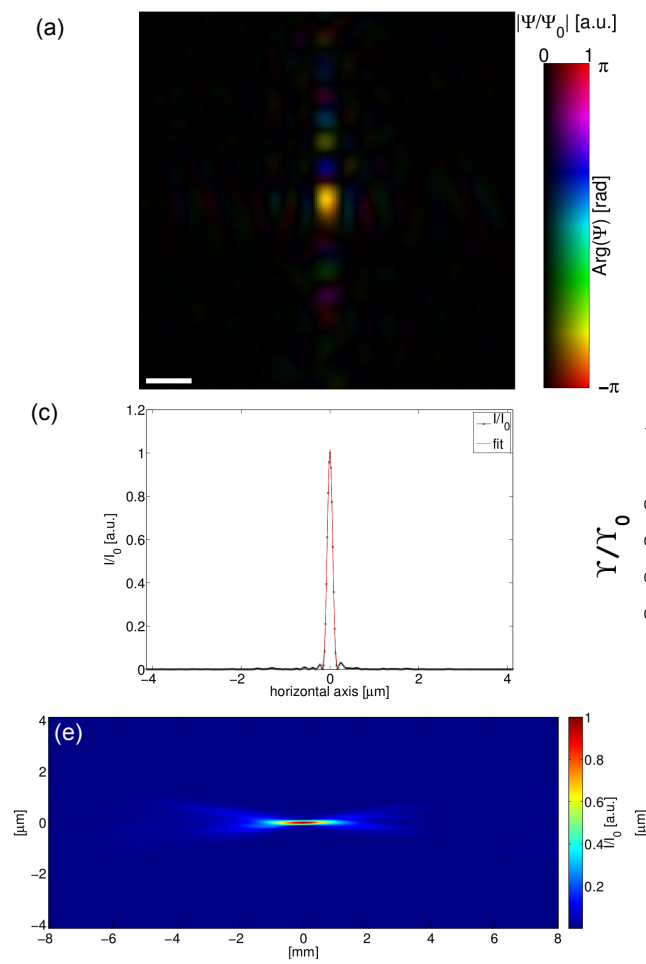

(b)

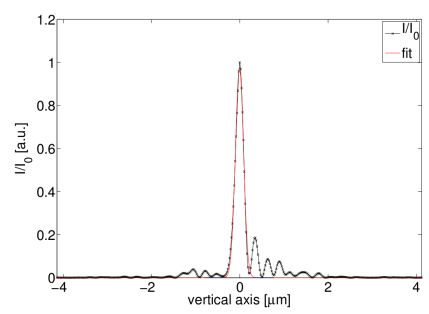

(d)
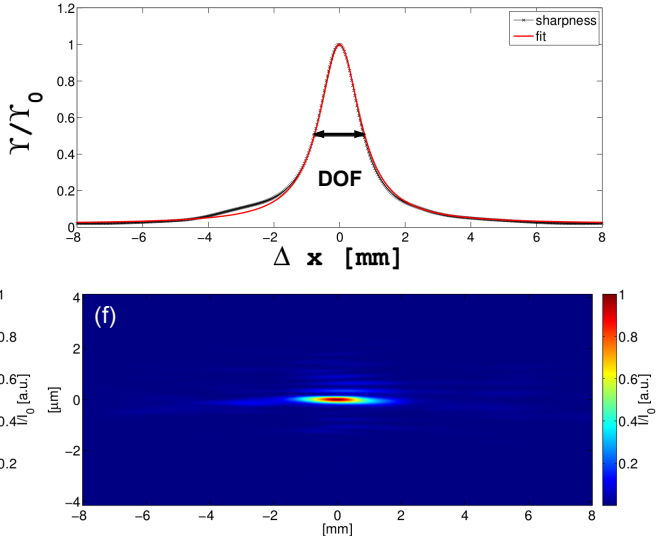

Figure 3.69: (a) complex field of the probe of the high photon flux dataset 3 (cf. Tab. 3.12 and Fig. 3.71) propagated to the focal plane. Amplitude and phase are drawn according to the colourbar next to the image. In (b) and (c) line-cuts through the vertical and horizontal direction of the probe intensity in the focus (a) are drawn, respectively. Gaussian fits to the curves (red lines) yield FWHM $=217 \mathrm{~nm}$ and FWHM $=136 \mathrm{~nm}$ in (b) and (c), respectively. (d) sharpness and fit for estimation of the DOF. (e) shows the horizontal slice of the probe intensity through the focus. (f) presents vertical slice. Scale bar in (a) denotes $500 \mathrm{~nm}$. 


\subsubsection{Results: Ptychographic Imaging of Nanowires}

At first, the results of the ptychographic reconstructions using a (coherent) photon flux of about $10^{7}$ phot/s and a small beam-defining slit gap S1 of $50 \mu \mathrm{m}$ are listed (Fig. 3.70). The image of the reconstructed phase of dataset 1 (Fig. 3.70 (a)) shows the phase shift of the standing nanowire (oriented perpendicular with the membrane, Fig. 3.67 (c)). A line cut through the phase reconstruction of the nanostructure is drawn in figure 3.70 (c). It is indicated in the original figure as dashed, white line. The maximum phase shift of the nanostructure in this graph is about $-0.24 \mathrm{rad}$ (after background subtraction). A Gaussian fit to the curve yields a width estimate of $305 \mathrm{~nm}$ (FWHM). A typical diffraction pattern of the KB beam imprint on the detector of this dataset is shown in figure 3.70 (b). The maximum count rate in the diffraction pattern is 91442 phot/s per pixel. It should be noted that the count rate is still below the maximum count rate of $3 \cdot 10^{5}$ phot/s per pixel of the Lambda detector. In figure 3.70 (d) the phase of the ptychographic reconstruction of a lying nanowire (oriented parallel to the membrane) can be clearly seen. The length is about $2.6 \mu \mathrm{m}$. The extend of the nanowire along the minor axis is in the range of $400-500 \mathrm{~nm}$. The phase shift was estimated from the reconstruction by averaging the phase map over small regions. An offset phase (black frame) was subtracted. On the body of the nanowire the average phase shift yields $\left\langle\Delta \Phi_{\text {body }}\right\rangle=(-0.034 \pm 0.001) \mathrm{rad}$ (white frame) and on the central part of the head the phase shift is increased in magnitude to $\left\langle\Delta \Phi_{\text {head }}\right\rangle=(-0.058 \pm 0.001) \mathrm{rad}$. The pixel size of both reconstructions is $52 \mathrm{~nm}$.

Next, the result of the ptychographic reconstructions using a (coherent) photon flux of $9.8 \cdot 10^{9} \mathrm{phot} / \mathrm{s}$ and a beam-defining slit gap S1 of $100 \mu \mathrm{m}$ is considered (figure 3.71). A representative diffraction pattern (after correction for the STCS attenuation) of dataset 3 is shown in figure 3.71 (a). In this setting the Lambda detector can record the diffracted intensity over more than 7 orders of magnitude. The KB imprint is obviously larger in comparison to the small S1 gap setting (cf. Fig. $3.70(b))$. It can be seen that borders of the central part of the beam have been placed close to the intermediate regions between neighbouring chips of the Lambda. Although rescaled, the regions between the chips can be identified in regions of low count rates. However, a smooth rescaling can be obtained if the count rate is sufficiently high. For instance, this can be seen where the tails of the KB beam extend over two chips (cf. Fig. 3.71 (b)). In addition, the high dynamic range of the signal reveals the fine features of the tails of the KB beam whose signal lies three to seven orders of magnitude below the central beam signal. Small oscillations in the range of a few pixels are characteristic and are highlighted by an enlarged representation (cf. Fig. 3.71 (b)).

The phase reconstruction of dataset 3 shows the lying nanowire of dataset 2 (Fig. 3.71 (c)). Here, the pixel size is $21 \mathrm{~nm}$. In comparison to the reconstruction of dataset 2 , the nanowire appears more smooth with less background. The resolution is visibly improved. In particluar, the shape of the head of the nanowire resembles more the shape that is observed in the SEM images (Fig. 3.67). A quantification 


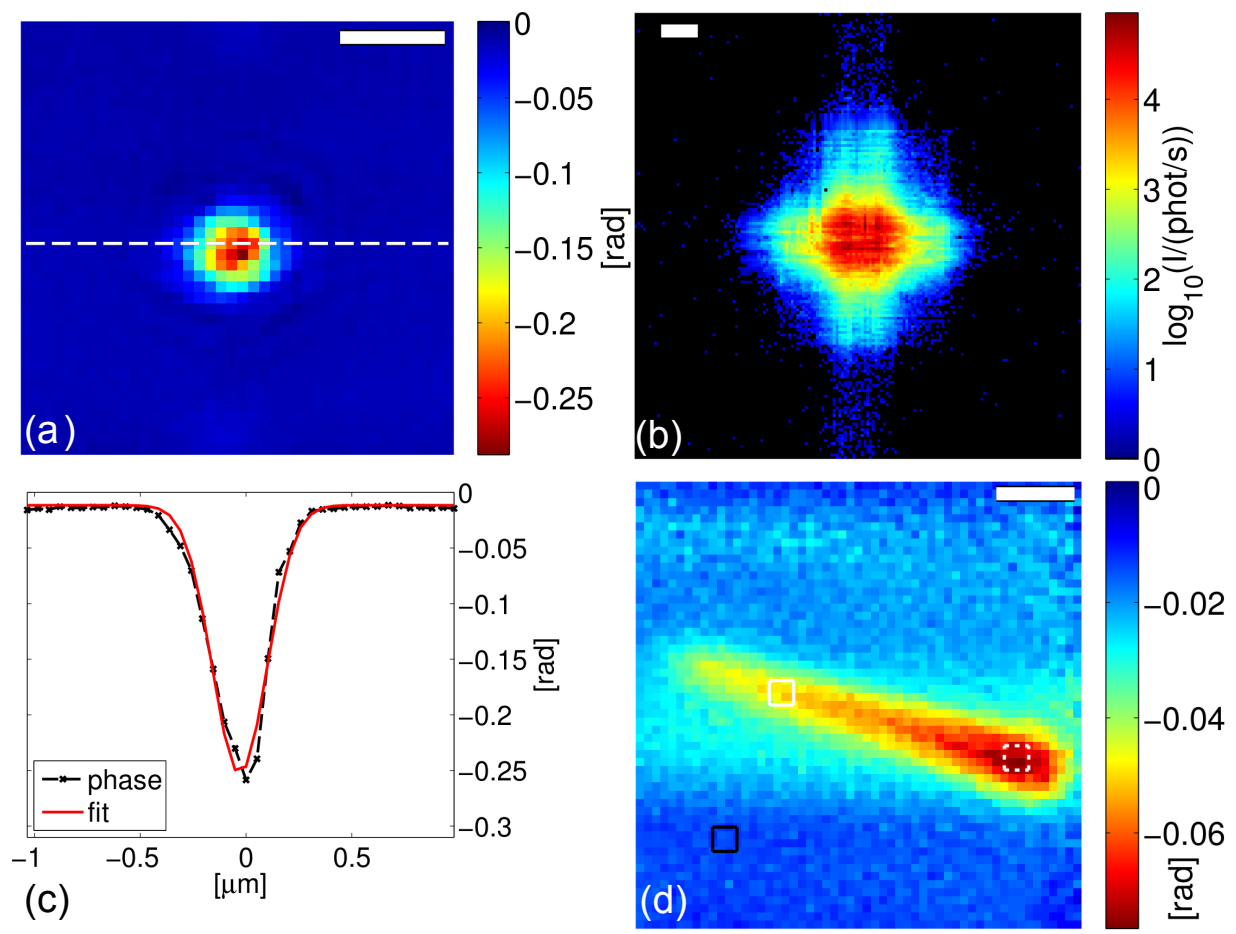

Figure 3.70: (a) phase reconstruction of the standing nanowire (dataset 1, Fig. 3.67 (c)). (b) shows a diffraction pattern of dataset 1. (c) presents a line cut through the phase reconstruction shown in (a) (black, dashed line). A fit of a Gaussian function (red line) to the phase profile of the nanowire yields an FWHM value of $305 \mathrm{~nm}$. (d) presents the phase reconstruction of a lying nanowire (dataset 2). The thicker top of the nanowire (cf. Fig. 3.67 (a), (c)) can be clearly identified by a larger phase shift which appears red in the image. The nanowire yields an average phase shift of $<\Delta \Phi_{\text {body }}>=(-0.034 \pm 0.001)$ rad on the body (white frame) and $\left\langle\Delta \Phi_{\text {head }}\right\rangle=$ $(-0.058 \pm 0.001) \mathrm{rad}$ on the head (dashed, white frame) after background subtraction (black frame). Scale bars in (a) and (d) denote $500 \mathrm{~nm}$, whereas the scale bar in (b) depicts $10 \mu \mathrm{m}^{-1}$. 
of the resolution follows in the next section. The measured phase shift on parts of the head (dashed, white frame) and the body (white frame) are $<\Delta \Phi_{\text {body }}>=$ $(-0.0350 \pm 0.0006) \mathrm{rad}$ and $\left\langle\Delta \Phi_{\text {head }}\right\rangle=(-0.0636 \pm 0.0006) \mathrm{rad}$, respectively. $\mathrm{A}$ line profile along the minor axis indicates the thickness of the nanowire (Fig. 3.71 (d)). The shape is well fitted by a Gaussian function with an FWHM $=247 \mathrm{~nm}$.

\subsubsection{Discussion, Conclusion \& Outlook}

Firstly, the ptychographic reconstructions of the wave-fields are discussed. The probe reconstruction allows to compare the $\mathrm{KB}$ wave-field between two different optical settings. A change of the slit gap setting causes a change of the size of the KB focus $[189]$. Here, the increase of the gap width of the beam defining slit by a factor of 2 could be shown to result in a decrease in size of the focus approximately by a factor of about 2 . The result is in agreement to Fourier optics where the focus size decreases linearly with an increase of the numerical aperture indicating full or a high degree of spatial coherence of the beam. In comparison to preceding experiments $\mid 96,98,332$, where typically a pinhole was used in front of the focal plane, the effectively pure KB beam, which is only slightly cleaned by the soft edge aperture, can be shown here. Moreover, the application of the STCS makes it possible to reconstruct the probe from high dynamic range intensity distributions, which cover about 7 orders of magnitude. The photon flux of $9.8 \cdot 10^{9} \mathrm{phot} / \mathrm{s}$ is still below the maximum coherent flux of about $10^{11}$ phot/s for larger slit gaps at the GINIX (cf. 258]) and thus limited by the geometric dimensions of the STCS which define the maximum slit gaps (cf. Fig. 3.65 (b)).

Next, the ptychographic reconstructions of the nanowires need to be considered. The phase shift of the lying nanowire should be comparable in both datasets 2 and 3 (Fig. 3.70 (d), 3.71 (c)). Indeed, a good agreement of the overall phase shift between both datasets is found. Moreover, the average phase shift over small parts of the body deviates less than $4 \%$ between both datasets. The slight deviation in the head region can be explained by the fact that the phase shift of a rod or cylindrically shaped object is not homogeneous which makes it difficult to define an average phase shift. In brief, the reconstructions are very consistent. The agreement in phase shift between the datasets holds also for the projection of the standing nanowire. From the SEM images a length of about $2.1 \mu \mathrm{m}$ and a thickness of about $0.3 \mu \mathrm{m}$ are inferred. Using the phase shift as determined from the ptychographic reconstruction of dataset 3 , an expected phase shift of $\Delta \Phi \approx(-0.0351 \cdot 4 / 5-0.0650 \cdot 1 / 5) \cdot(2.1 / 0.3) \mathrm{rad}=-0.29 \mathrm{rad}$ is obtained for a model nanowire that consists of $4 / 5$ of body and $1 / 5$ of head (both of thickness $0.3 \mu \mathrm{m}$ ) which is in good agreement to the reconstruction (Fig. 3.70 (a)). However, a discrepancy in comparison to tabulated values is noted. For instance, the typical width of the InP core of the nanowires shown here is not below $150 \mathrm{~nm}$, which already yields a phase shift of $-0.048 \mathrm{rad}\left(\rho_{\mathrm{InP}}=4.79 \mathrm{~g} / \mathrm{cm}^{3}\right)$ at $13.8 \mathrm{keV} \mid 125$. It is known that a wrong rescaling of the STCS can induce errors in the phase shift 332 (cf. section 3.3 p. 132). On the other hand, the consistency of the two phase maps (with and without STCS) is a good quality control of the STCS 

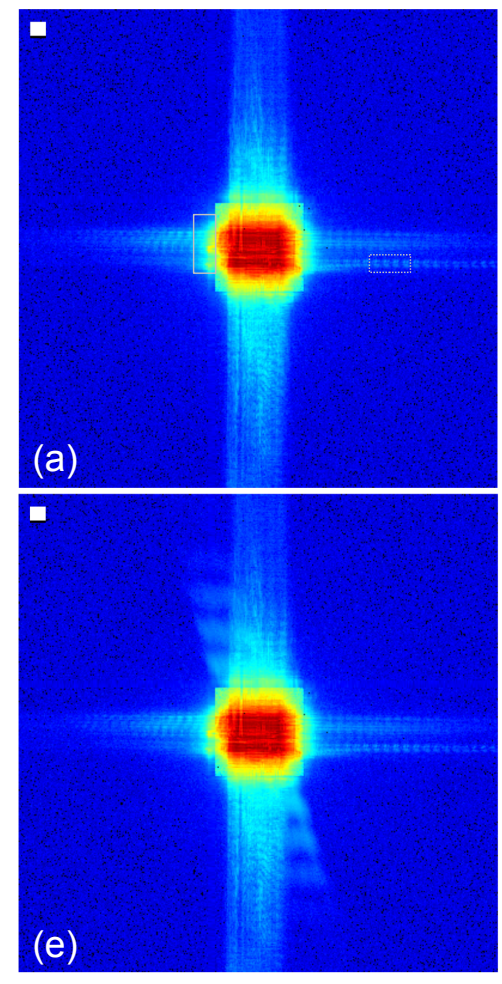

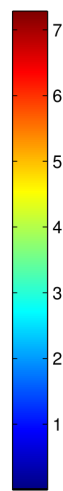

$\widehat{\widehat{m}}(\mathrm{~b})$
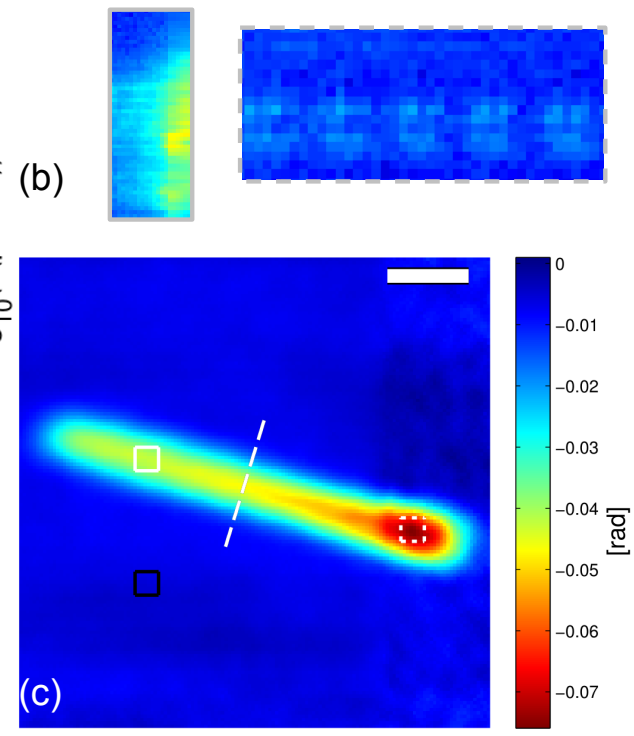

(d)

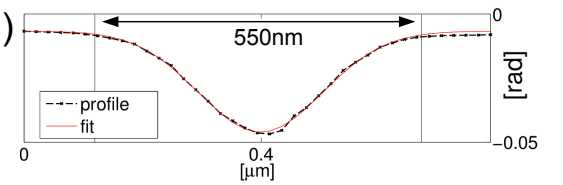

Figure 3.71: (a) shows a diffraction pattern of dataset 3 . The central part of the diffraction pattern has already been rescaled by the attenuation mask of the STCS. (b) shows enlarged parts of the diffraction pattern in (a). The intermediate region between adjacent chips where the remapping of the recorded intensity from larger to smaller pixels yields good results due to high photon count rates can be seen on the region with solid, grey frame. The other region highlights the high frequency oscillations of the KB farfield on the detector (dashed, grey frame). (c) presents recontructed phase of the lying nanowire corresponding to the data recording using the STCS (dataset 3). The nanowire is the same as in dataset 2 (Fig. 3.70 (d)). It yields an average phase shift of $\left\langle\Delta \Phi_{\text {body }}\right\rangle=(-0.0350 \pm 0.0006)$ rad on the body (white frame) and $<\Delta \Phi_{\text {head }}>=(-0.0636 \pm 0.0006)$ rad on the head (dashed, white frame) after background subtraction (black frame). (d) shows the line profile in (c) as indicated by a dashed, white line. A Gaussian (FWHM $=247 \mathrm{~nm})$ is well fitted to the profile. (e) shows a diffraction pattern with scattering contributions from the nanowire. Scale bars in (a), (e) and (c) denote $10 \mu \mathrm{m}^{-1}$ and $500 \mathrm{~nm}$, respectively. 
rescaling. Therefore, one can be confident about the quantitativeness of the ptychographic reconstructions with respect to the STCS rescaling. Consequently, the measurement of the attenuation profile can be an alternative to determine the optimum rescaling factor for the STCS attenuated region. In this context, it should also be noted that a single factor as was used in $[332$ may not be applicable here because the determined profile is not homogeneous (cf. Fig. 3.66).

Finally, the improvement of resolution due to the gain in photon flux from dataset 2 to 3 needs to be addressed. Here a visible improvement of the reconstruction in terms of resolution and background could be achieved. However, it is also noted that the diameter of the nanowire in the reconstruction is larger than expected from SEM images (Fig. 3.67), that give about $300-350 \mathrm{~nm}$. A comparison of the $2 \mathrm{~d}$ power spectral densities (PSD) of the reconstructed phase maps (cf. Fig. 3.72) reveals that most information of the nanowire (the shape) is contained within a frequency ring that corresponds to a resolution of about $125 \mathrm{~nm}$. In case of dataset 3 , the inner frequency part appears to be slightly larger but there are also contributions for structure sizes of $38 \mathrm{~nm}$ (cf. white arrow in Fig. 3.72 (b)). Another estimate of the resolution that also estimates the correctness of the phasing of the algorithm can be obtained by calculating the 'phase-retrieval transfer function' (PRTF) (cf. $|97| 274,274$ and equation 3.111, p. 82). The PRTFs of dataset 1 (red) and 2 (green) are very similar. Both curves drop below 0.5 at about $\nu=6 \mathrm{\mu m}^{-1}$ (half-period) indicating that the resolution of these datasets is not better than $\mathrm{dx}=1 / 2 \nu=83 \mathrm{~nm}$. In contrast, the PRTF of dataset 3 (blue) remains close to unity without any significant descent. It should be noted that this only indicates a resolution of the probe field in the range of the pixel size $(\mathrm{dx}=21 \mathrm{~nm})$, i.e. the contributions of the object are 'hidden' 62,97 . The resolution of the object cannot be better than $38 \mathrm{~nm}$ as the PSD analysis revealed. As can be seen by comparing the images of figures 3.70 (d) and 3.71 (c), little more information about the structure of the nanowires could be obtained. For instance, in the optimal case one would be able to observe a deviation from the smooth Gaussian curve across the nanowire rod (cf. Fig. 3.71 (d)) due to the different layers of material.

Next, the increase in fluence, which can be used to estimate the potential gain in resolution due to the increase in dose, is discussed. The dose $D$ depends on the photon energy $E$, the linear absorption coefficient $\mu$, the mass density $\rho_{m}$ and the fluence $F[141]$. The overlap between adjacent illuminated spots of the ptychographic data is accounted for according to equation 3.120 (p. 92). Here, the fluence on the sample per single recorded diffraction pattern is defined by taking only the photons of the reconstructed single probe intensity $I_{P}$ around the central peak into account, i.e. $\rho_{\gamma}(\vec{r})=I_{P}$ and $\chi$ (cf. 3.120, p. 92) corresponds to an ellipse whose minor and major axes are defined by the half width at half maximum $(\mathrm{FWHM} / 2)$ of the intensity distribution. The fluence of the low flux dataset 2 is estimated to be $F \approx 5 \cdot 10^{1} \mathrm{phot} / \mathrm{nm}^{2}$ and the high flux dataset 3 yields $F \approx 1 \cdot 10^{5} \mathrm{phot} / \mathrm{nm}^{2}$ (equiv. a flux density of $10^{5} \mathrm{phot} / \mathrm{nm}^{2} / \mathrm{s}$ ). On the other hand, the overall average fluence over the area of the sample which accounts for the overlap (eqn. 3.120 ) yields an even higher fluence of $F \approx 6 \cdot 10^{5} \mathrm{phot} / \mathrm{nm}^{2}$ 

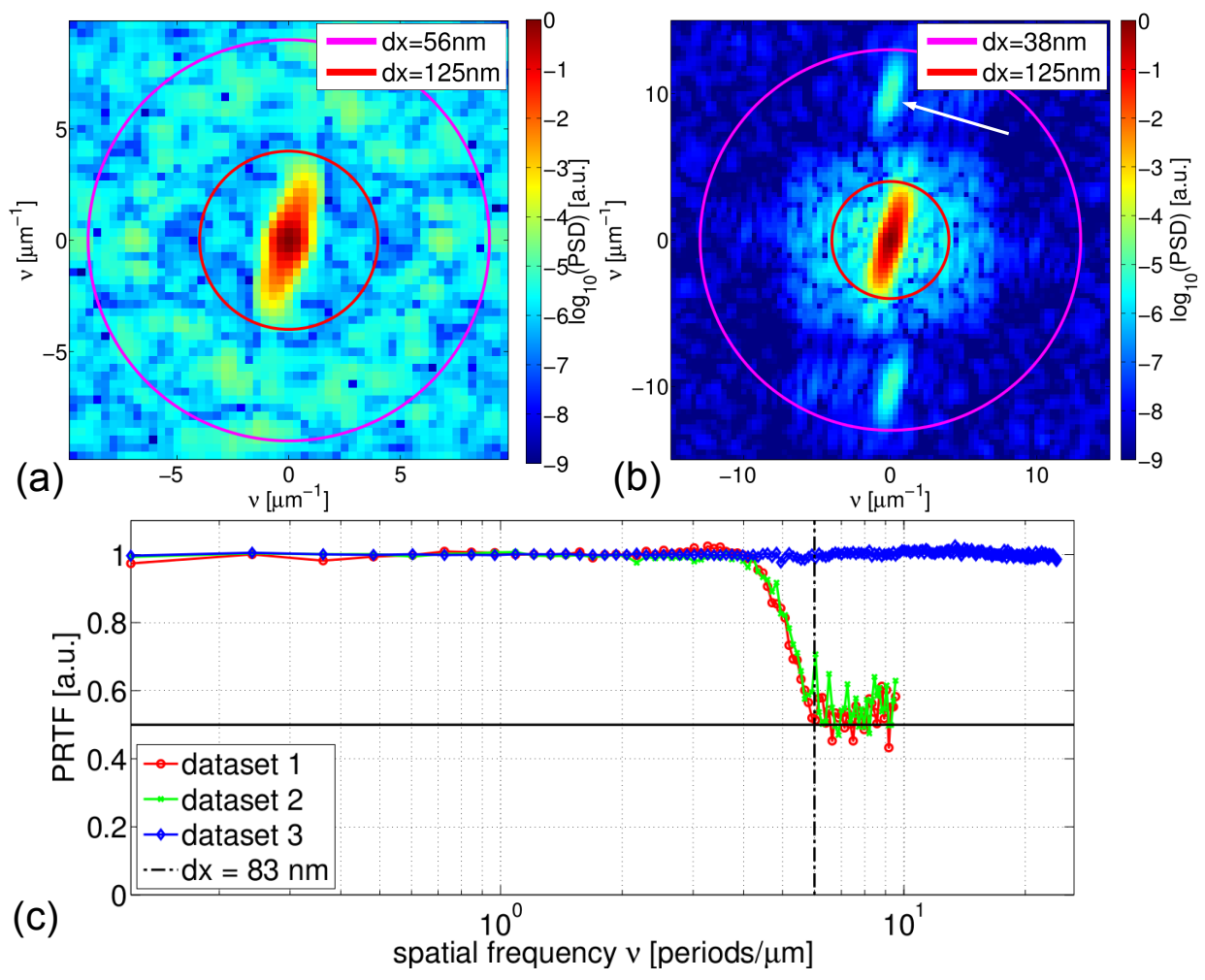

Figure 3.72: (a) and (b) show 2 d power spectral densities of the phase reconstructions of dataset 2 and 3 (cf. Fig. 3.70 (d) and 3.71 (c)), respectively. The frequency contributions from the rod shape of the nanowire lie within a frequency ring that corresponds to $\mathrm{dx}=1 / 2 \nu=125 \mathrm{~nm}$ half period resolution. In the power spectrum of dataset 3 (b) frequency contributions appear also in the frequency shell corresponding to $\mathrm{dx}=38-125 \mathrm{~nm}$ (cf. white arrow). (c) presents PRTF calculations of the three datasets: 1 (red), 2 (green), 3 (blue). The drop of the PRTF below 0.5 (horizontal, solid black line) is indicated by a vertical, dash-dotted black line at $\mathrm{dx}=83 \mathrm{~nm}$ half period resolution. 
for dataset 3. Hence, the STCS in combination with the beam resizing allows to increase the fluence on the sample by $3-4$ orders of magnitude. At first, one notes that the fluence obtained here is quite high. For instance, $2.75 \cdot 10^{4} \mathrm{phot} / \mathrm{nm}^{2}$ has been reported by 266 using $15.25 \mathrm{keV}$ X-rays focused down to $\sim 80 \mathrm{~nm} \times 80 \mathrm{~nm}$ (smaller by a factor of 5). Schropp and co-workers estimated their resolution on a $500 \mathrm{~nm}$ thick, binary tantalum test structure to be about $10 \mathrm{~nm}$. Hence, at least on test structures with sharp edges, the potential resolution should be in the range of $10 \mathrm{~nm}$, which is in good agreement to preceding experiments [332]. One reason explaining the discrepancy between expected and observed resolution would be to attribute the result to non-ideal experimental parameters such as effects of partial coherence. However, the PRTF of dataset 3 indicates that at least the probe reconstruction appears to be very good up to the highest frequency. Hence, vibrations of the sample as discussed by e.g. Clark et al. [43 may explain our results, also with respect to the observed deviation of the phase shift of the nanowire from tabulated values. Another degrading factor could be fluorescence radiation from the Ge STCS (emission lines $K_{\alpha_{1}}=9.89 \mathrm{keV}, K_{\alpha_{2}}=9.86 \mathrm{keV}$, $\left.K_{\beta_{1}}=10.98 \mathrm{keV}[302]\right)^{25}$. In addition, a supposedly minor effect may be small sensitivity variations of the pixels of the Lambda detector [260, which could be compensated by a flatfield correction.

In conclusion, a powerful ptychographical experimental setting is presented that paves the way for experiments with unprecedented flux density. First ptychographic reconstructions using the new Lambda detector were shown. The use of a higher energy of $13.8 \mathrm{keV}$ is an improvement as future experiments investigating elemental constituents of the sample will take advantage of the wider range of available $K$-edges such as $\mathrm{Ni}, \mathrm{Cu}, \mathrm{Zn}$ and $\mathrm{Br}$ in comparison to earlier experiments using $7.9 \mathrm{keV}$. In addition, the reconstructions clearly benefit from the effectively non-missing data problem between adjacent chips of the detector, which is a common problem for many other pixel detectors. The high count rate of the Lambda detector in combination with the STCS and the reconstruction of the probe field has enabled us to set up a fluence-optimised ptychographic setting. As far as could be deduced from other works in this field, the flux density of about $10^{5} \mathrm{phot} / \mathrm{nm}^{2} / \mathrm{s}$ is the highest one that has been reported at synchrotron storage rings for ptychographic imaging experiments in combination with photon counting pixel detectors (cf. Tab. 3.13). In addition, there are two more features of the Lambda detector that have not been addressed in these experiments but which may become important for following experiments. Firstly, the small pixel size of $55 \mu \mathrm{m}$ in comparison to e.g. $172 \mu \mathrm{m}$ (Pilatus, Dectris) relaxes oversampling requirements. In simple terms, oversampling limits the ratio between beamsize and field of view (FOV) in the sample plane per single diffraction pattern 195. According to the small angle approximation the FOV per single diffraction pattern depends on the wavelength $\lambda$, the distance between sample and detector $X$ and the pixel size of the detector $D$, i.e. $\mathrm{FOV}=\lambda X / D$. The smaller pixel size thus allows a large field of view (here $\sim 8 \mu \mathrm{m} \times 8 \mu \mathrm{m}$ ), which in principle opens the possibility for

\footnotetext{
${ }^{25}$ Note that the emission lines lie well above $7.9 \mathrm{keV}$ but the transmission for $100 \mu \mathrm{m}$ hardly differs (cf. Tab. G.2 p. 198 which allows the use of this STCS at $7.9 \mathrm{keV}$.
} 
large beam experiments. Consequently, the total scanned area of the sample can be increased while the number of scan points is kept constant. The small pixel size also alleviates the use of higher energies (or smaller distances between sample and detector). Secondly, the $10^{10} \mathrm{phot} / \mathrm{s}$ of the beam can also be distributed in favour of shorter exposure times. Thereby reduced scanning times would presumably increase the quality of reconstructions due to a decrease of thermal drifts and vibrations. The Lambda can be operated in a continuous read-write mode and it provides a read-out of about 2000 frames/s. Hence, the detector is very well suited for short exposure times, and it may become important in experiments where deadtime is crucial.

Table 3.13: The table lists flux density achievements in ptychographic imaging experiments. Estimations have been obtained according to the reported beamsize (cf. text).

\begin{tabular}{rll}
\hline $\begin{array}{r}\text { flux density } \\
\left.\text { [phot } / \mathrm{nm}^{2} / \mathrm{s}\right]\end{array}$ & Beamline & Reference \\
\hline & & \\
$1.0 \cdot 10^{5}$ & P10 PETRAIII & this work \\
$7.50 \cdot 10^{3}$ & P10 PETRAIII & Giewekemeyer et al. 2014 96$]$ \\
$2.26 \cdot 10^{2}$ & P10 PETRAIII & Wilke et al. 2013 [332] \\
$1.83 \cdot 10^{4}$ & P06 PETRAIII & Schropp et al. 2012 [266 \\
$2.7 \cdot 10^{3}$ & cSAXS SLS & Guizar-Sicairos et al. 2012 \\
$2.74 \cdot 10^{1}$ & BL29XUL SPring-8 & Takahashi et al. 2011 $[287$ \\
$0.21 \cdot 10^{0}$ & cSAXS SLS & Giewekemeyer et al. 2010|97. \\
\hline
\end{tabular}




\subsection{Waveguide-Based Imaging of $B$. thuringiensis and $B$. subtilis}

Quantitative waveguide-based X-ray imaging has been carried out on the level of single, unstained, unsliced and freeze-dried bacterial cells of B. thuringiensis and B. subtilis using hard X-rays of $7.9 \mathrm{keV}$ photon energy. The cells have been especially prepared in a state where they occur in their metabolically dormant form of an endospore. The obtained data allows for mass and mass density determinations on the level of single individual endospores but includes also large field of view investigations. Depending on the field of view, a resolution down to $65 \mathrm{~nm}$ was achieved for quantitative phase reconstructions.

X-ray waveguides $[209,228$ are optical elements for preparing quasi point-sources with sizes well below $100 \mathrm{~nm}$ in the hard X-ray regime [147, 165, 221]. Due to the filtering of the coupled X-rays by propagation through the waveguide $[91$, 222 , the prepared quasi X-ray point source is ideally suited for X-ray imaging in the holographic regime [89,165]. In addition, the waveguide source can also be used for iterative phase retrieval of isolated biological specimens in two and three dimensions $[12-14]$ by taking advantage of robust phase retrieval in the Fresnel regime $94,241,334,335$. Moreover, imaging of biological specimens such as bacteria that are prone to radiation damage during the measurement process in this regime is considered to be rather dose efficient [13, 14,241.

Before addressing the experimental results, details about the experimental set-up, sample preparation and theory are given. In particular, the theoretical background of the holographic imaging regime is reviewed briefly. The section closes by summarising the results and a comparison of the mass measurements with results from other works from different fields. Martin Krenkels' support for the CTF-based reconstructions is gratefully acknowledged. Parts of this section have been prepared for publication [329].

\subsubsection{Experimental Set-up}

The waveguide-based imaging experiments were carried out during two successive beamtimes with the GINIX set-up at the P10 coherence beamline of the PETRA III synchrotron located at DESY, Hamburg in Germany. Monochromatic X-ray undulator radiation with an energy of $7.9 \mathrm{keV}$ was selected by using a double crystal monochromator $(\mathrm{Si}(111))$. The beam was focused by a pair of X-ray mirrors in the Kirkpatrick-Baez geometry (cf. Fig. 3.73 and e.g. section 3.3, p. 132, for details). The beam was then reduced by a waveguide whose guiding channel (air surrounded by silicon) was aligned with respect to the KB focus using a hexapod (SmarAct, Germany). The waveguide was fabricated by e-beam lithography. The channel dimensions are $d_{h} \times d_{v}=97 \mathrm{~nm} \times 73 \mathrm{~nm}$ on the entrance side (cf. 'G23' in $[114]$ ). The channel length is about $1 \mathrm{~mm}$. The source size on the exit of the waveguide is about $25 \mathrm{~nm} \times 31 \mathrm{~nm}(\mathrm{FWHM}, \mathrm{h} \times \mathrm{v})[13$. The sample stage follows behind the waveguide. It consisted of a high precision piezo-electric stage (PI, Germany/ SmarAct, Germany) on top of coarse stepper motors (Micos). The 
sample is typically positioned a few millimetres at a distance $z_{01}$ to the waveguide exit. An evacuated flight-tube of $5 \mathrm{~m}$ length was installed behind the sample stage to minimize scattering effects of $\mathrm{X}$-rays in air. The imaging detector used for collecting the waveguide diffraction data is based on a sCMOS camera (Photonic Science, GB) for visible light. X-ray radiation is detected by visible light from a custom scintillator ( $15 \mu \mathrm{m}$ GdOS:Tb, Photonic Science) that is fiber-coupled to the chip. The detector has $1920 \times 1080$ pixels $(\mathrm{h} \times \mathrm{v})$ with a pixel size of $6.5 \mu \mathrm{m}$. The distances between waveguide and detector were either $z_{02}=5.04 \mathrm{~m}$ or $z_{02}=$ $5.13 \mathrm{~m}$, depending on the beamtime. In addition, the single photon-counting, zero read-out noise pixel detector Pilatus 300K (Dectris, Switzerland) could be inserted at the same distance.

\subsubsection{Sample Preparation}

The Bacillus subtilis (DSM no. 23778) and Bacillus thuringiensis (DSM no. 350) samples have been cultivated as detailed in section 2.3.2 (p. 57). Briefly, cell material and endospores are harvested after 6 days of incubation at $30^{\circ} \mathrm{C}(200 \mathrm{rpm})$. Excess medium was removed by centrifugation at $3000 \mathrm{~g}$ for $10 \mathrm{~min}$ at $4{ }^{\circ} \mathrm{C}$. Thereafter, the pellets were washed two times with pure water (centrifugation at $5000 \mathrm{~g}$ for $10 \mathrm{~min}$ ). In case of the B. subtilis material, a layer consisting of almost solely endospores could be extracted after the second centrifugation step. It appears as a dark band on top of the cell material. The sample material was then stored at low temperature $4-8{ }^{\circ} \mathrm{C}$ until further use. Part of the material was further processed for preparation of TEM samples as described in section 2.3 .3 (p. 59). TEM images are already presented in Figs. 2.17 (p. 53) and 2.18 (p. 54).

The samples for X-ray imaging were prepared by suspending about $10-20 \mu \mathrm{l}$ of the pellet in $100 \mu \mathrm{l}$ pure water (for an optimum material density on the sample holder). Next, the samples were plunge-frozen using a vitrobot (Leica EM GP, Leica Microsystems). Droplets of $1 \mu \mathrm{l}$ cell/ endospore suspension were placed on $\mathrm{Si}_{3} \mathrm{~N}_{4}$ membranes (Silson, UK; membrane thickness $1 \mu \mathrm{m}$ ) inside a humidity and temperature controlled chamber $\left(R_{h} \approx 70 \%, T \approx 4^{\circ} \mathrm{C}\right)$ of the vitrobot. After blotting excess liquid, the samples were plunged into the cryogen of $63 \%$ propane, $37 \%$ ethane $\left(T=-193^{\circ} \mathrm{C}\right) \mid 305$. Samples were freeze-dried in a workshop-build freeze drying apparatus. A microscopic inspection of the sample material used for plunge-freezing was carried out after the plunging procedure (cf. Fig. 3.74) and after freeze-drying (cf. Figs. 3.75, 3.76). 


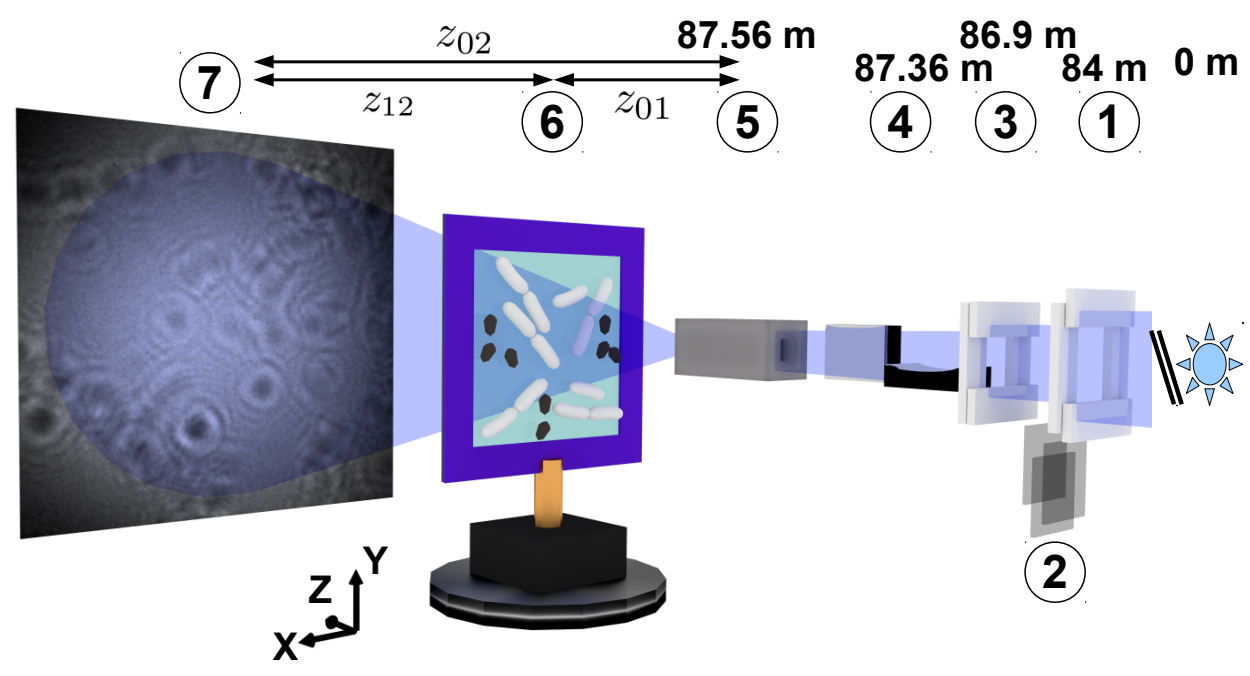

Figure 3.73: Schematic of the GINIX waveguide set-up: downstream of the undulator source and the monochromator (not shown) (1) slits S1, (2) attenuation foils made of $\mathrm{Al},(3)$ slits S2, (4) KB-mirror system, (5) X-ray waveguide (entrance in the focal plane of the KB-mirrors), (6) bacterial sample at a distance $z_{01}$ to the waveguide and (7) detection device at a distance $z_{02}$ to the waveguide. The flight-tube between sample and detector is not shown in the image.

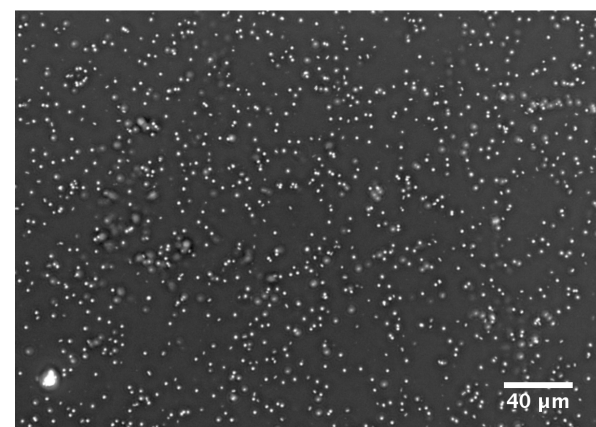

(a)

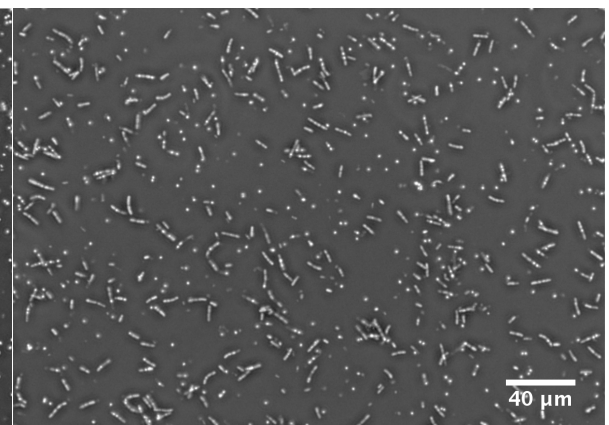

(b)

Figure 3.74: Optical light micrographs (phase contrast, 40x) of (a) B. subtilis and (b) $B$. thuringiensis suspension used for plunge-freezing. In (a) almost only endospores which appear as phase-bright spots are present. In (b) the endospores are visible outside the cells but supposedly also inside some of the rod-like cells where they appear as phase-bright spots. 


\subsubsection{Details: Theory, Data Recording \& Data Treatment}

At first, the theoretical background for waveguide-based imaging is addressed briefly. The diffraction pattern $I\left(\overrightarrow{\rho^{\prime}}, z_{02}\right)$ of the object transmission function $O\left(\vec{\rho}, z_{01}\right)$ being placed in the divergent beam of a waveguide $P\left(\vec{\rho}, z_{01}\right)$ can be described at the detection plane $z_{02}$ by the Fresnel diffraction integral

$$
I\left(\overrightarrow{\rho^{\prime}}, z_{02}\right)=\left|\mathfrak{F}_{\mathfrak{Q}}\{P O\}\left(\overrightarrow{\rho^{\prime}}, z_{02}\right)\right|^{2}
$$

Due to the small size of the waveguide exit, the waveguide beam can be considered quasi point-like $\mid 90,165,166$. That is, $P\left(\vec{\rho}, z_{01}\right) \approx\left|P\left(\vec{\rho}, z_{01}\right)\right| \exp \left(i k \vec{\rho}^{2} /\left(2 z_{01}\right)\right)$. According to the Fresnel-Scaling theorem [223, the diffracted intensity can be described in an effective geometry of coordinates $\overrightarrow{\rho^{\prime}} / M$ and $z_{e}$ (cf. $12,90,166$ )

$$
I\left(\overrightarrow{\rho^{\prime}}, z_{02}\right)=\left|\mathfrak{F}_{\mathfrak{Q}}\{P O\}\left(\overrightarrow{\rho^{\prime}}, z_{02}\right)\right|^{2} \approx\left|\mathfrak{F}_{\mathfrak{Q}}\{|P| O\}\left(\overrightarrow{\rho^{\prime}} / M, z_{e}\right)\right|^{2}
$$

where the geometric magnification $M$ of the system and the effective propagation distance $z_{e}$ are given by

$$
\begin{aligned}
M & =1+\frac{z_{01}}{z_{12}} \approx \frac{z_{12}}{z_{01}} \quad \text { and } \\
\frac{1}{z_{e}} & =\frac{1}{z_{01}}+\frac{1}{z_{12}} \equiv \frac{M}{z_{12}} .
\end{aligned}
$$

Typically, equation $(3.128)$ is further simplified by the approximation 94,95

$$
\begin{aligned}
I\left(\overrightarrow{\rho^{\prime}}, z_{02}\right) & \approx\left|\mathfrak{F}_{\mathfrak{Q}}\{|P| O\}\left(\overrightarrow{\rho^{\prime}} / M, z_{e}\right)\right|^{2} \\
& \approx\left|\mathfrak{F}_{\mathfrak{Q}}\{|P|\}\left(\overrightarrow{\rho^{\prime}} / M, z_{e}\right) \mathfrak{F}_{\mathfrak{Q}}\{O\}\left(\overrightarrow{\rho^{\prime}} / M, z_{e}\right)\right|^{2} \\
& \equiv I_{e} \cdot I_{h},
\end{aligned}
$$

where $I_{e}=\left|\mathfrak{F}_{\mathfrak{Q}}\{P\}\left(\overrightarrow{\rho^{\prime}} / M, z_{e}\right)\right|^{2}$ denotes the empty beam intensity and $I_{h}=$ $\left|\mathfrak{F}_{\mathfrak{Q}}\{O\}\left(\overrightarrow{\rho^{\prime}} / M, z_{e}\right)\right|^{2}$ is the near-field hologram of the object. A prerequisite for reconstruction of the waveguide illuminated near-field diffraction data is to record an accurate diffraction pattern of the empty beam. Using equation (3.132), the holographic data of the object is obtained by dividing the raw data $I\left(\overrightarrow{\rho^{\prime}}, z_{02}\right)$ by the empty beam intensity $I_{e}, 12,14,94,95,115,166$

$$
I_{h} \approx I\left(\overrightarrow{\rho^{\prime}}, z_{02}\right) / I_{e}
$$

As shown in 115], the accuracy of the empty beam division crucially depends on the smoothness of the illuminating beam. Here, it should be noted that the far-field of the waveguides is indeed very smooth (cf. [90,114, 166, 212]). For the present experiment, the empty beam division is exemplified in Fig. 3.77 presenting $I, I_{e}$ and the obtained holographic intensity $I_{h}$.

Next, the recorded data is listed. Single diffraction patterns and series of diffraction patterns with a corresponding series of empty beam intensities has been recorded at different source to sample distances $z_{01}$ (equivalent to a variation 
of the magnification) on different positions of both Bacillus spp. samples. The experimental parameters are summarised in tables 3.14 and 3.15 . In addition, the diffraction data of the $B$. thuringiensis sample includes a transversal variation of the sample position. On region (A), $M=126$, the sample is illuminated at four different overlapping regions by shifting the sample thrice by about $150 \mu \mathrm{m}$ in the horizontal direction.

In the following, details about the iterative phase reconstruction and the determination of the support being necessary for the $\mathrm{mHIO}$ algorithm are described. In case of the $B$. thuringiensis a semi-automatic procedure could be applied to obtain an accurate support function. Let $g_{x}, g_{y}$ be the gradient of the holographically reconstructed phase, then it was found that the 'radial' gradient $g=\sqrt{g_{x}^{2}+g_{y}^{2}}$ information can be used to locate the cellular structures in the holographic reconstructions as exemplified in Fig. 3.78a. A first support estimate is obtained by creating a binary mask from $g$ by setting a threshold, followed by a dilation of the binary data and, finally, filtering (Fig. 3.78b). The final support was then obtained similarly but by using a preliminary iterative reconstruction with the first support guess. In case of the B. subtilis data, the gradient method was not found to be practical due to the low gradient values of the sample in comparison to the holographic background. Instead, a simple threshold for the holographically reconstructed phase is set, followed by dilation and filtering of the binary mask. The reconstructions were carried out at a maximum of 2000 iterations using the parameters $\beta=\gamma=0.2$ (cf. section 1.3.5, p. 39). The parameter $D$ of the softprojection onto the modulus of the measured diffraction data was set manually. Here it should be noted that if $D$ is chosen too small the algorithm clearly diverges within a few hundred iterations. The optimum $D$ is thus found by taking a slightly larger value above the threshold where divergence is observed. A comparison of $D$ with the calculated error at the last iteration showed a good agreement for most of the data (cf. Tabs. H.1 and H.2, p. 198 and 199). The error distributions of the other cases do not show a clear divergent behaviour. For the present analysis, their corresponding $D$ parameter was not further optimised. Prior to merging the $B$. thuringiensis data that was taken at four different positions on region (A) at constant $z_{01}$, the displacement vectors between the neighbouring phase reconstruction were calculated by using the method of discrete Fourier transform registration 113 .

The second phase reconstruction method that has been applied here is known as 'holotomography' $45,47,340$. The name arises from the imaging regime where it can be applied, namely the holographic regime, and from its typical combination with tomography. The underlying phase reconstruction scheme is based on reconstructing two-dimensional projection data that not necessarily involves an extension to three-dimensional datasets. The phase reconstruction is based on the contrast transfer function. Hence, the reconstruction method will be referred to as CTF-based in the following. In the case of a pure phase object with slowly 
varying phase, the Fourier transform of the measured intensity in the plane $z$ can be approximated by (here the one-dimensional case is given for simplicity) 257

$$
\mathcal{F}\left\{I_{z}\right\}(\nu) \approx \delta_{D}(\nu)+2 \sin \left(\pi \lambda z \nu^{2}\right) \mathcal{F}\{\phi\}(\nu),
$$

where $\mathcal{F}\{\phi\}(\nu)$ is the Fourier transform of the phase of the object $\phi=-k \int \delta d z$ (cf. equation 1.69, p. 26) and $\delta_{D}$ denotes the Dirac delta function. The sinusoidal term has a significant influence on the transfer of the phase of the object in terms of spatial frequencies. For instance, phase contrast of the object is strongly suppressed at the zeros $\nu_{n}=\sqrt{n / \lambda z}, n \in \mathbb{N}_{0}$. The dependence of the contrast on the propagation distance suggests to include measurements from multiple distances $z_{m}$. The Fourier representation of the phase function can be obtained by minimising the following cost function 340

$$
S_{c}[\tilde{\phi}]=\frac{1}{N} \sum_{m=1}^{N} \int_{0}^{\infty} d \nu\left|\tilde{I}_{z_{m}}^{(\exp )}-\tilde{I}_{z_{m}}^{(t)}\right|^{2},
$$

where the tilde denotes the Fourier transform and the summation is over $N$ distances $z_{m} . \tilde{I}_{z_{m}}^{(e x p)}$ and $\tilde{I}_{z_{m}}^{(t)}$ denote the experimental data and the theoretical model according to equation 3.134 , respectively. Minimising the cost function with respect to $\tilde{\phi}$, i.e. requiring $\partial_{\tilde{\phi}} S_{c}=0$, yields the Fourier transform of the phase function 340

$$
\tilde{\phi}(\nu)=\frac{\sum_{m} \tilde{I}_{z_{m}}^{(e x p)} \sin \left(\pi \lambda z_{m} \nu^{2}\right)}{\sum_{m} 2 \sin ^{2}\left(\pi \lambda z_{m} \nu^{2}\right)} .
$$

The phase $\phi$ is thus obtained by an inverse Fourier transform of the right-hand side. In practice, this involves a regularisation parameter in the denominator that is frequency dependent and also determined by the experiment.

Next, the determination of the applied dose during the measurements is discussed. The photon flux was measured with the Pilatus 300K during both experiments. The photon fluxes corresponding to the smaller field of view of the Photonic Science detector were calculated to be $1.43 \cdot 10^{8}$ photons $/ \mathrm{s}$ and $2 \cdot 10^{8}$ photons $/ \mathrm{s}$ in case of the $B$. thuringiensis and the B. subtilis data, respectively. Doses were then determined by assuming the photon flux to be constant over the field of view and taking the tabulated absorption coefficient for the model protein $\mathrm{H}_{50} \mathrm{C}_{30} \mathrm{~N}_{9} \mathrm{O}_{10} \mathrm{~S}_{1}$ of density $\rho=1.35 \mathrm{~g} / \mathrm{cm}^{3}[141$ (cf. equation 3.120, p. 92). Results are listed in Tabs. 3.14, 3.15.

Calculation of the projected two-dimensional mass densities $\sigma_{m}$ from the reconstructed phase maps were performed according to equation 3.121 (p. 100, see also section 1.2 , p. 20). A $10 \%$ error according to [97 was assumed for the integral mass values $( \pm 20 \mathrm{fg})$. 
Table 3.14: Experimental parameters such as the distance between source and sample $z_{01}$ and the achieved magnification $M$ of the $B$. thuringiensis data are listed. The distance $z_{02}$ between sample and detector was in all cases $z_{02}=5.04 \mathrm{~m}$. $N$ is the number of images that were recorded. The integral doses of the reconstructions of Figs. 3.793 .85 and 3.86 are on the order of $3 \cdot 10^{3} \mathrm{~Gy}$ (mean overlap of 2 adjacent reconstructions), $2 \cdot 10^{5} \mathrm{~Gy}$ and $4 \cdot 10^{5} \mathrm{~Gy}$, respectively.

\begin{tabular}{lllllll}
\hline Region & Figure & $\begin{array}{l}z_{01} \\
{[\mathrm{~mm}]}\end{array}$ & $M$ & $\begin{array}{l}N \cdot \Delta T \\
{[\mathrm{~s}]}\end{array}$ & $\begin{array}{l}\text { Fluence } \\
{\left[\mathrm{phot} / \mathrm{nm}^{2}\right]}\end{array}$ & $\begin{array}{l}\text { Dose } \\
{[\mathrm{Gy}]}\end{array}$ \\
\hline (A) & & 40 & 126 & $5 \cdot 5$ & $1.27 \cdot 10^{0}$ & $1.66 \cdot 10^{3}$ \\
(A) & 3.79 & 40 & 126 & $5 \cdot 5$ & $1.27 \cdot 10^{0}$ & $1.66 \cdot 10^{3}$ \\
(A) & 40 & 126 & $5 \cdot 5$ & $1.27 \cdot 10^{0}$ & $1.66 \cdot 10^{3}$ \\
(A) & & 40 & 126 & $5 \cdot 5$ & $1.27 \cdot 10^{0}$ & $1.66 \cdot 10^{3}$ \\
(B) & 3.80 & 15.80 & 319 & $10 \cdot 10$ & $3.26 \cdot 10^{1}$ & $4.25 \cdot 10^{4}$ \\
(C) & 3.81 & 5.30 & 952 & $1 \cdot 10$ & $2.11 \cdot 10^{1}$ & $2.76 \cdot 10^{4}$ \\
\hline (B) & & 13.70 & 369 & $10 \cdot 10$ & $4.33 \cdot 10^{1}$ & $5.65 \cdot 10^{4}$ \\
(B) & 3.85 & 13.80 & 366 & $10 \cdot 10$ & $4.27 \cdot 10^{1}$ & $5.57 \cdot 10^{4}$ \\
(B) & & 14.80 & 341 & $10 \cdot 10$ & $3.71 \cdot 10^{1}$ & $4.84 \cdot 10^{4}$ \\
(B) & & 15.80 & 319 & $10 \cdot 10$ & $3.26 \cdot 10^{1}$ & $4.25 \cdot 10^{4}$ \\
(C) & 3.86 & 5.30 & 952 & $10 \cdot 5$ & $1.45 \cdot 10^{2}$ & $1.89 \cdot 10^{5}$ \\
(C) & & 5.40 & 935 & $10 \cdot 5$ & $1.39 \cdot 10^{2}$ & $1.82 \cdot 10^{5}$ \\
\hline & & & & & & \\
\end{tabular}

Table 3.15: Experimental parameters such as the distance between source and sample $z_{01}$ and the achieved magnification $M$ of the $B$. subtilis data are listed. The distance $z_{02}$ between waveguide and detector was in all cases $z_{02}=5.13 \mathrm{~m}$. The integral dose for the CTF-based reconstruction scheme (Fig. 3.87) is on the order of $4 \cdot 10^{5} \mathrm{~Gy}$.

\begin{tabular}{lllllll}
\hline Region & Figure & $z_{01}[\mathrm{~mm}]$ & $M$ & $\Delta T[\mathrm{~s}]$ & Fluence $\left[\mathrm{phot} / \mathrm{nm}^{2}\right]$ & Dose $[\mathrm{Gy}]$ \\
\hline (A) & $3.83 \mathrm{a}$ & 3.56 & 1442 & 5 & $4.63 \cdot 10^{1}$ & $6.05 \cdot 10^{4}$ \\
(B) & $\overline{3.83 \mathrm{~b}}$ & 1.81 & 2836 & 8 & $2.87 \cdot 10^{2}$ & $3.75 \cdot 10^{5}$ \\
(C) & $3.83 \mathrm{c}$ & 1.81 & 2836 & 8 & $2.87 \cdot 10^{2}$ & $3.75 \cdot 10^{5}$ \\
(D) & $3.83 \mathrm{~d}$ & 2.56 & 2005 & 8 & $1.43 \cdot 10^{2}$ & $1.87 \cdot 10^{5}$ \\
\hline (A) & & 2.31 & 2222 & 5 & $1.10 \cdot 10^{2}$ & $1.44 \cdot 10^{5}$ \\
(A) & 3.87 & 2.56 & 2005 & 5 & $8.96 \cdot 10^{1}$ & $1.17 \cdot 10^{5}$ \\
(A) & & 2.81 & 1827 & 5 & $7.44 \cdot 10^{1}$ & $9.71 \cdot 10^{4}$ \\
(A) & & 3.06 & 1677 & 5 & $6.27 \cdot 10^{1}$ & $8.19 \cdot 10^{4}$ \\
\hline
\end{tabular}




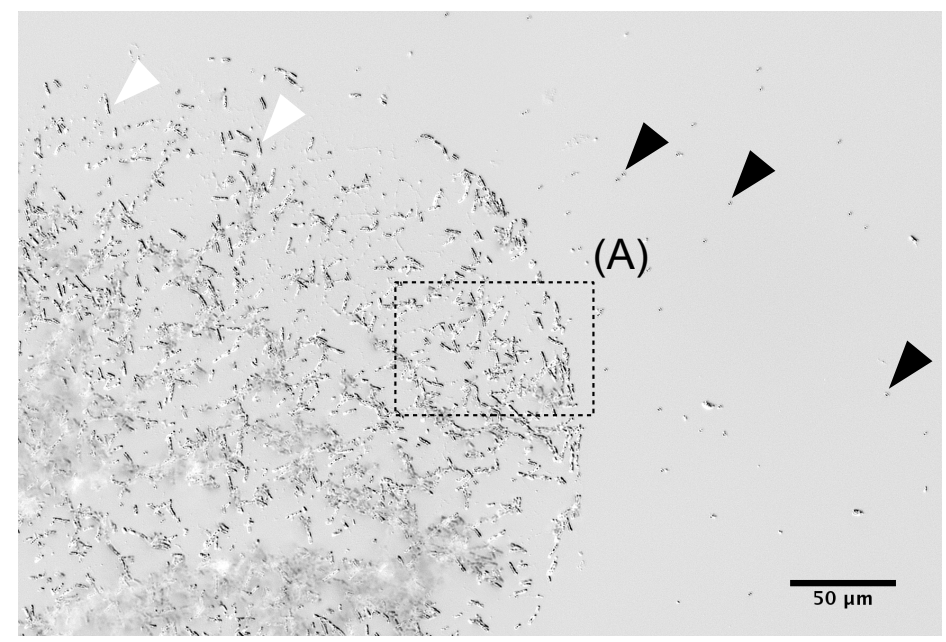

(a)

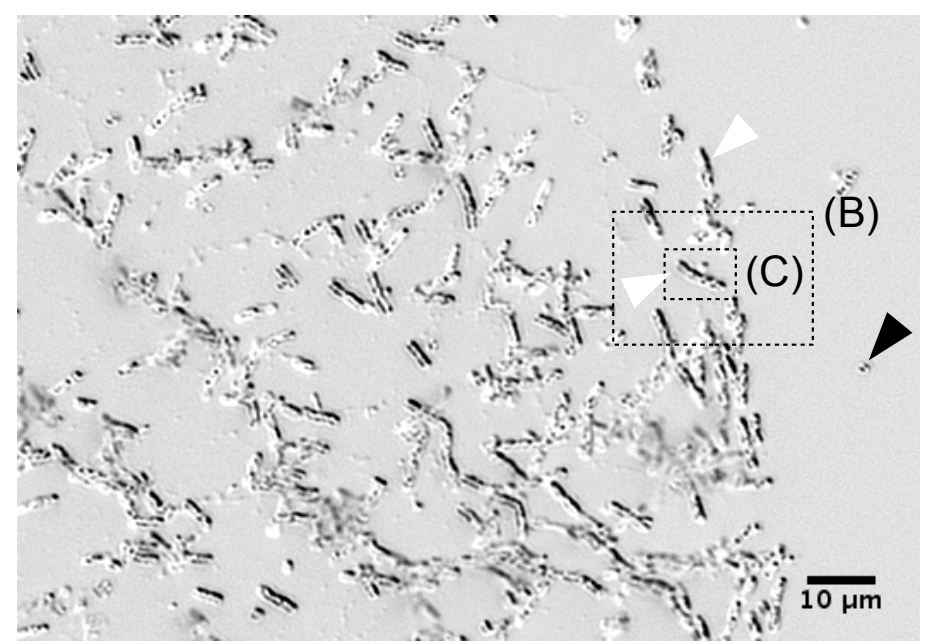

(b)

Figure 3.75: (a) Optical light micrograph (DIC, 40x) of the sample of freeze-dried B. thuringiensis cells and endospores (cf. Fig. 2.27 p. 68). (b) close-up of that region. The regions that were analysed with waveguide based imaging are indicated by a dashed, black frames and labels (A), (B), (C) in both images. The bacteria and remnant cell material occurs mainly rod-shaped (white arrow heads). Smaller spots are likely isolated endospores (black arrow heads). 


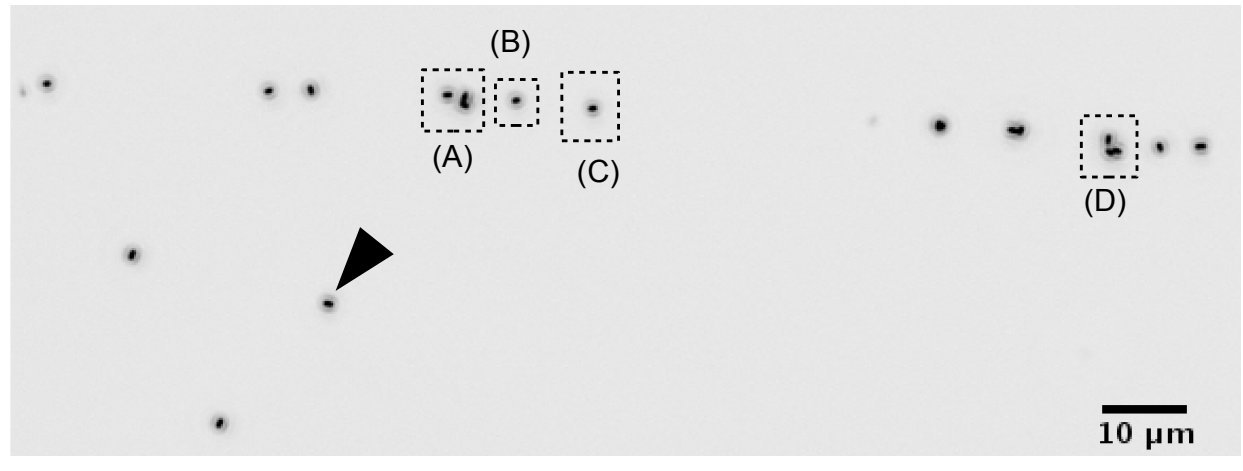

Figure 3.76: Optical light micrograph (DIC, 40x) of the sample of freeze-dried $B$. subtilis endospores (cf. Fig. 2.26 p. 67). The regions that were analysed with waveguide based imaging are indicated by a dashed, black frames. These are labelled (A), (B), (C) and (D). Endospores are indicated by a black arrow head. 


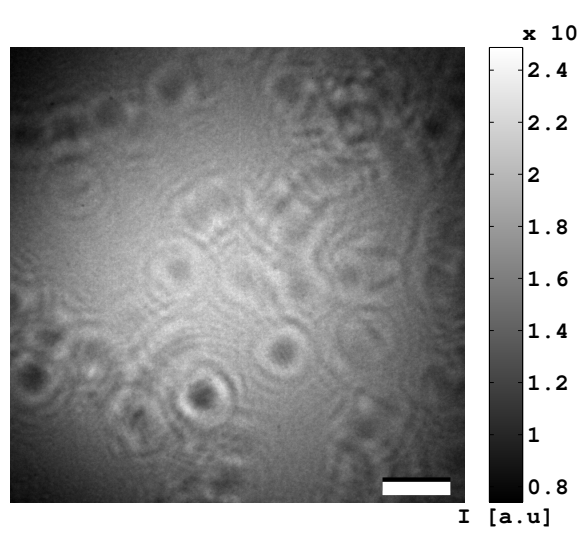

(a)

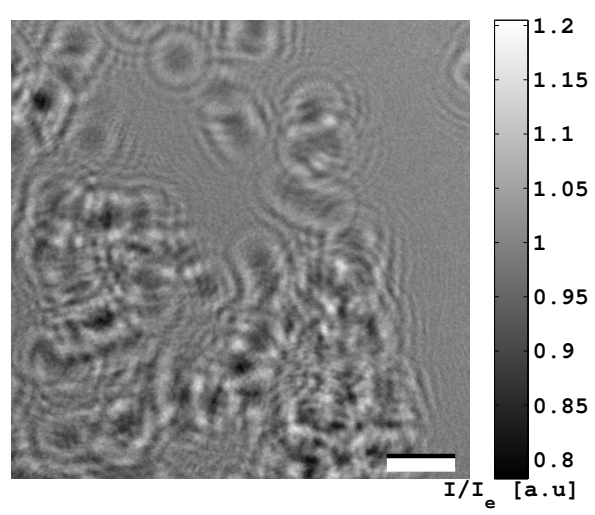

(c)

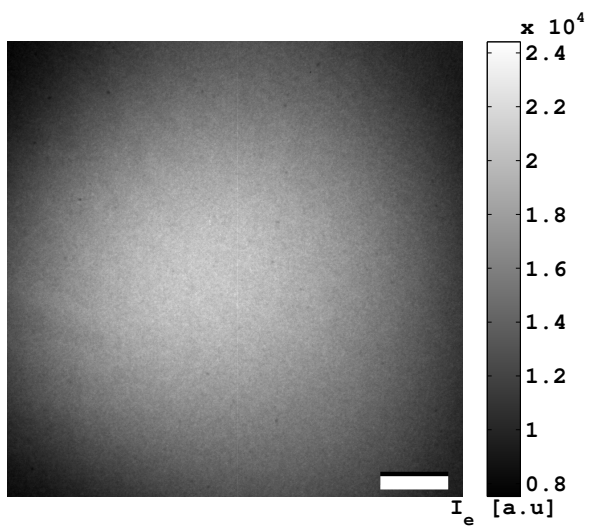

(b)

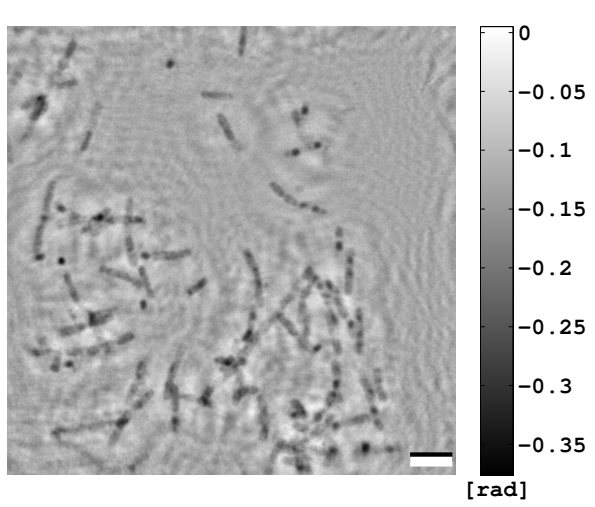

(d)

Figure 3.77: Data of one position on region (A) of sample B. thuringiensis (cf. table 3.14): (a) recorded intensity $I$, (b) empty beam intensity $I_{e}$, (c) hologram $I_{h}=$ $I / I_{e}$ and (d) phase of holographic reconstruction. The holographic reconstruction yields already a good impression of the sample structure. For instance, the rodshaped bacteria (or their remnant empty sheaths) can be indentified. However, the reconstruction appears distorted due to the twin image problem. Scale bars (a), (b) (c) and (d) denote $1 \mathrm{~mm}$ (real detector dimensions) and $5 \mu \mathrm{m}$ (effective geometry), respectively. 


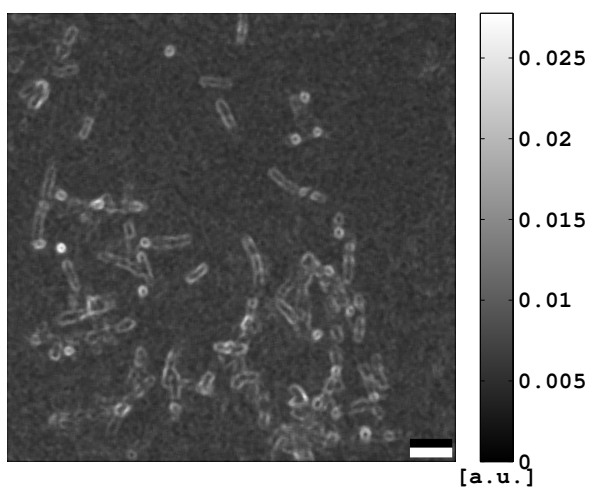

(a)

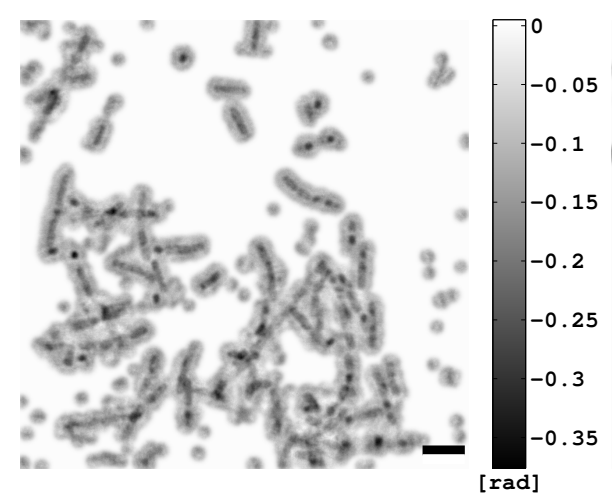

(b)

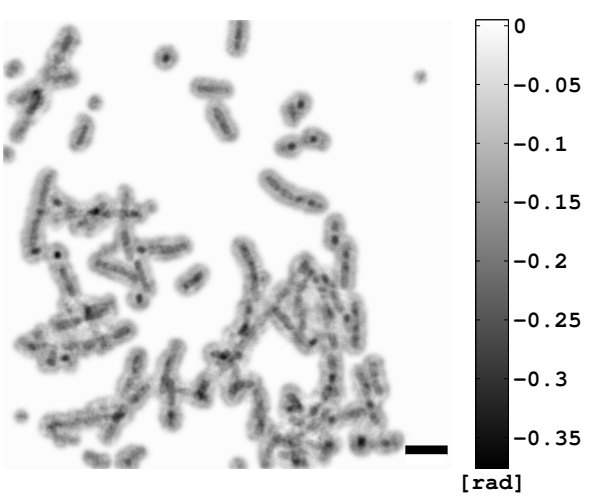

(c)

Figure 3.78: (a) shows the 'radial' gradient of the holographic reconstruction (Fig. 3.77 d). (b) product of first support determination and holographic reconstruction. (c) same as (b) but using the final support. Scale bars denote $5 \mu \mathrm{m}$. 


\subsubsection{Results}

At first, the results of the $\mathrm{mHIO}$ reconstructions of the $B$. thuringiensis data are addressed. Three regions (A), (B) and (C) (cf. optical light micrographs of Fig. 3.75 ) on the sample of the unstained, unsliced and freeze-dried bacterial material were successfully reconstructed. Fig. 3.79 shows the phase map corresponding to four single mHIO reconstructions. Together, the full field of view covers about $100 \mu \mathrm{m} \times 50 \mu \mathrm{m}$ at a magnification of $M=126$ (Tab. 3.14). A resolution estimate from the power spectral density (PSD) of a large part of the phase map $(1024 \times 1024$ pixels) is presented in Fig. 3.82a. Most of the frequency contributions are contained inside a ring of $238 \mathrm{~nm}$ half period resolution but small fractions appear also in the region down to about $100 \mathrm{~nm}$. The sample structure correlates very well with the optical light micrographs. Many remnants of the rod-like bacterial cells can be identified with varying contrast. Single cells abundantly appear to be connected in strands of two or more cells. Due to the prepared state of the cell culture (late stationary phase) many of the cells may already be lysing or empty. The calculated projected mass density (phase shift) shows cells with different density or multiple cells lying on top of each other. In addition, some smaller spots within the cells exhibit a comparably high mass density $\left(>0.14 \mathrm{mg} / \mathrm{cm}^{2}\right)$. On this sample the high mass density objects are supposedly endospores or depending on the size possibly protein crystals as will become clear after discussing the phase maps with higher resolution.

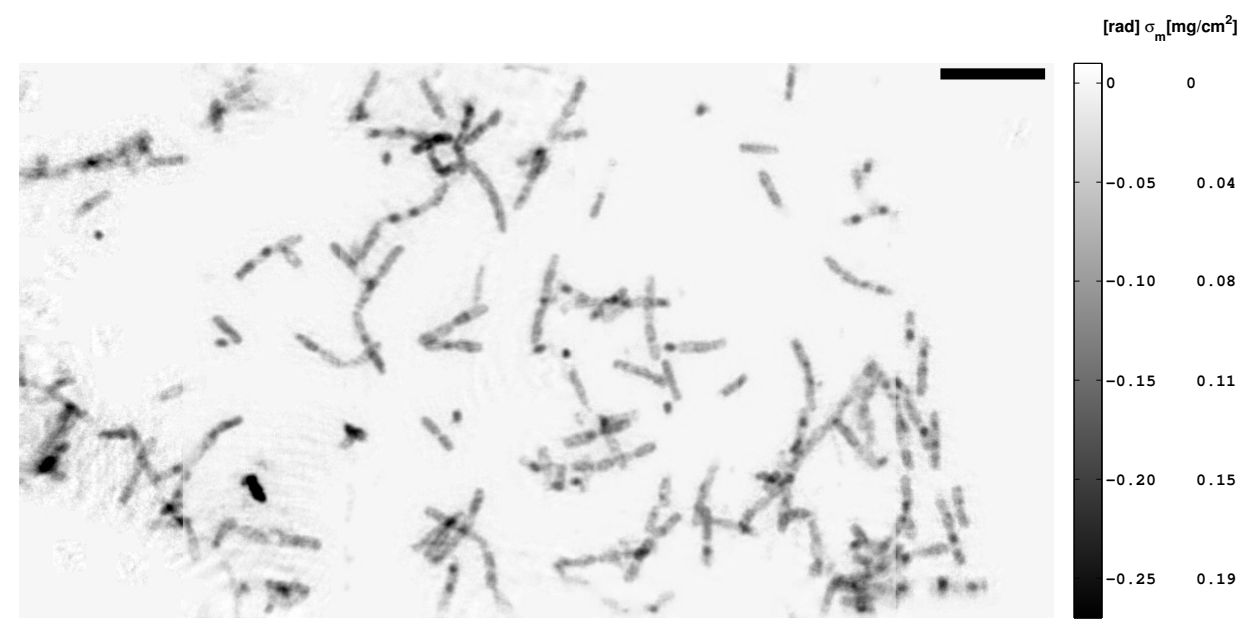

Figure 3.79: Region (A): The image shows a phase map of the $B$. thuringiensis sample. The phase map corresponds to 4 single mHIO reconstructions in the same plane that have been merged. The scale bar denotes $10 \mu \mathrm{m}$.

The mass density map of region (B) which was taken at a magnification of $M=$ 319 is presented in Fig. 3.80. Its power spectral density (Fig. 3.82b) indicates that the overall resolution is about $150 \mathrm{~nm}$, extending to about $90 \mathrm{~nm}$ (half period). 
On the mass density map five ellipsoid structures were attributed to intracellular endospores (labelled as (1)-(5)). The mass of these endospores was determined by integrating the projected mass density map as indicated by black dots surrounding the endospores. Material of the cell walls below and on top of the endospores was accounted for by subtracting a background of $39 \mathrm{fg}$ which was obtained from the same map. As a result, the dry masses of individual endospores are obtained in the range of $(113-181) \pm 20 \mathrm{fg}$ with an average mass of $\left\langle m_{B . t h u}\right\rangle=153 \pm 9 \mathrm{fg}$ for a single, dry endospore (Tab. 3.16). In addition to endospores, other smaller dense features are visible on the mass density map as indicated by black arrow heads in Fig. 3.80. These features may well be indicative of the BT-crystals formed by $B$. thuringiensis as can be observed on the TEM images (e.g. Fig. 2.18, p. 54).

Another reconstructed mass density map taken on region (C) at a magnification of $M=952$ is shown in Fig. 3.81. Its power spectral density (Fig. 3.82c) indicates a resolution down to $100 \mathrm{~nm}$ (half period) and slightly better. In comparison to the preceding reconstruction of Fig. 3.80 , the resolution is clearly improved. A strand of four partly lysed bacterial cells can be identified. Two of these cells include dense features (cf. black arrow heads) like the one, that was labelled as endospore (3), which may be attributed to the BT-crystals.

Table 3.16: Results of mass measurements of individual endospores of $B$. thuringiensis are presented below. The average mass per single endospore is $\left\langle m_{B . t h u}\right\rangle=153 \pm 9 \mathrm{fg}$.

\begin{tabular}{llll}
\hline Region & Figure & Label & Mass [fg] \\
\hline & & 1 & $181 \pm 20$ \\
& & 2 & $166 \pm 20$ \\
(B) & 3.80 & 3 & $130 \pm 20$ \\
& & 4 & $174 \pm 20$ \\
& & 5 & $113 \pm 20$ \\
\hline
\end{tabular}




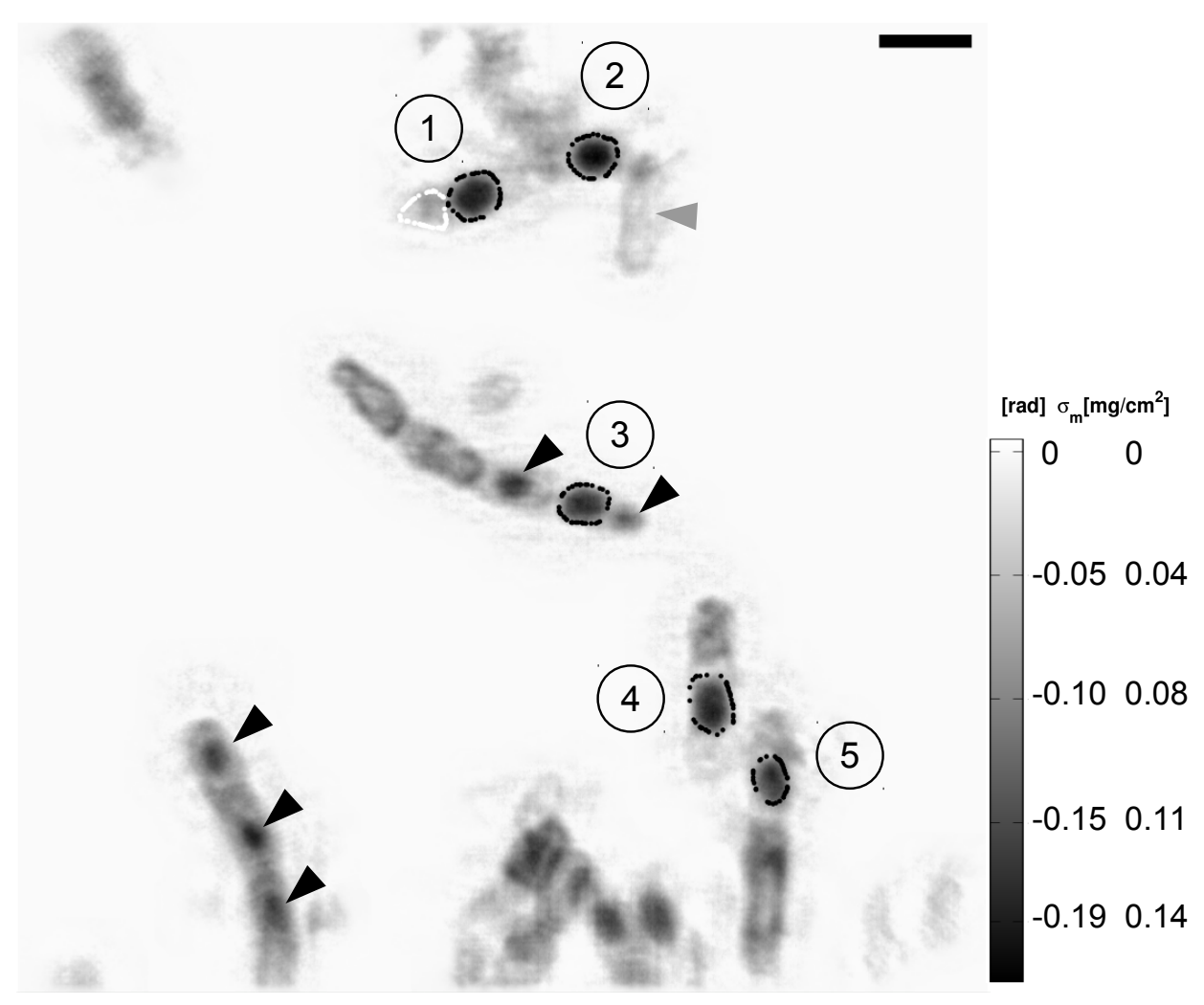

Figure 3.80: Region (B): The image shows a phase image of the $B$. thuringiensis sample. The phase map corresponds to a single reconstruction. The black dots indicate the boundaries of the endospores used for mass estimations whereas the white dots surround the region being defined here as 'background' $\left(\left\langle m_{B G}\right\rangle=39 \mathrm{fg}\right)$. Endospores are labelled from 1 to 5 . The average mass per single endospore is $\left\langle m_{B . t h u}\right\rangle=153 \pm 9 \mathrm{fg}$. The black arrow heads indicate positions of possibly other dense bacterial features such as BT-crystals. The grey arrow head points to an almost fully lysed cell. The scale bar denotes $2 \mu \mathrm{m}$. 


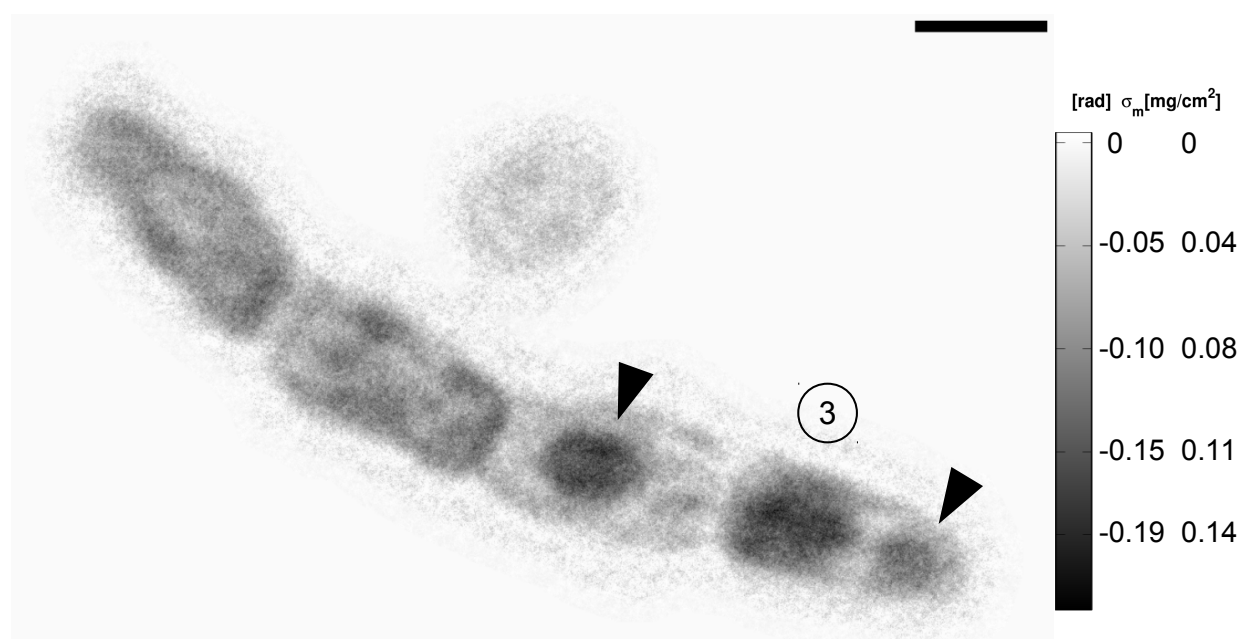

Figure 3.81: Region $(\mathrm{C})$ : The image shows a phase image of the $B$. thuringiensis sample. The phase map corresponds to a single reconstruction. The scale bar denotes $1 \mu \mathrm{m}$. 


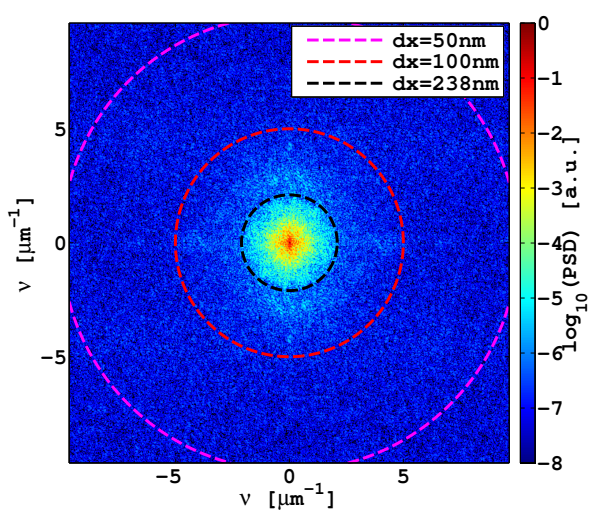

(a)

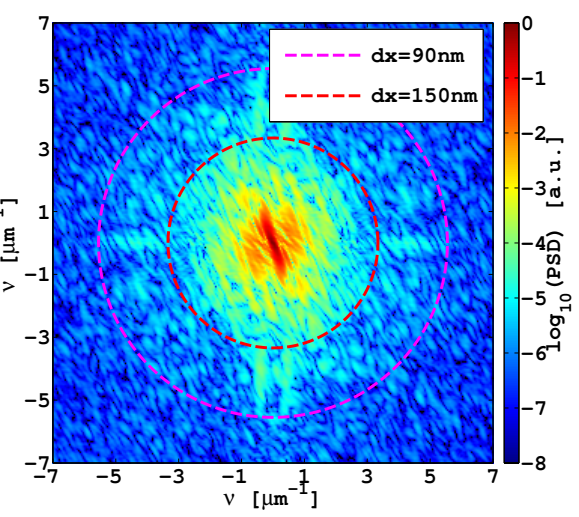

(b)

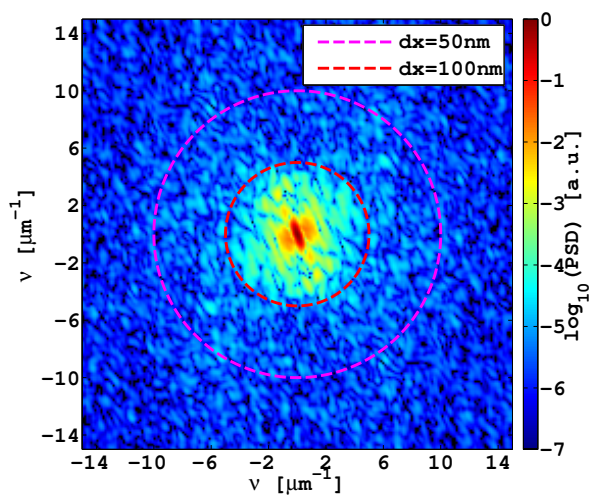

(c)

Figure 3.82: The figure presents calculated two-dimensional PSDs of the mHIO phase reconstructions of the $B$. thuringiensis data. (a) PSD of reconstruction of region (A) (Fig. 3.79), (b) PSD of reconstruction of region (B) (Fig. 3.80) and (c) PSD of reconstruction of region (C) (Fig. 3.81) indicating resolutions in the range of $238 \mathrm{~nm}$, $150 \mathrm{~nm}$ and $100 \mathrm{~nm}$, respectively. 
Next, the results of the mHIO reconstructions of the $B$. subtilis data from regions (A), (B), (C) and (D) are given. The optical light micrograph of Fig. 3.76 clearly shows isolated endospores on all these regions. The phase reconstructions are summarised in Fig. 3.83. In total, eight individual endospores can clearly be identified (labels (1)-(8)). Their projected mass density extends up to $0.19 \mathrm{mg} / \mathrm{cm}^{2}$. The endospores appear without the cell material of the mother cell as expected. For this reason the determination of the endospore masses can be obtained without background subtraction. Mass results of the dried endospores are listed in Tab. 3.17 and are in the range of $(153-192) \pm 20 \mathrm{fg}$. The average mass per single dried endospore is $\left\langle m_{\text {B.sub. }}\right\rangle=170 \pm 7 \mathrm{fg}$. Notably, the endospores differ slightly in shape and size. The resolution of the reconstruction was estimated by calculation of the power spectral densities of the phase maps of Figs. $3.83 \mathrm{a}, 3.83 \mathrm{~b}$. The twodimensional PSDs are presented in Fig. 3.84 which show frequency contributions down to a resolution of $100 \mathrm{~nm}$ (half period). Internal structure of the endospores cannot clearly be identified. However, the inner parts of the endospores appear slightly denser after a small transition zone which may indicate the transition from coat to cortex and the centre of the endospore.

Table 3.17: Results of mass measurements of individual endospores of B. subtilis are listed below. The average mass per single endospore is $\left\langle m_{B . s u b .}\right\rangle=170 \pm 7 \mathrm{fg}$.

\begin{tabular}{llll}
\hline Region & Figure & Label & Mass [fg] \\
\hline \multirow{3}{*}{ (A) } & & 1 & $153 \pm 20$ \\
& $3.83 \mathrm{a}$ & 2 & $180 \pm 20$ \\
& & 3 & $192 \pm 20$ \\
\hline (B) & $3.83 \mathrm{~b}$ & 4 & $158 \pm 20$ \\
\hline (C) & $3.83 \mathrm{c}$ & 5 & $154 \pm 20$ \\
\hline \multirow{3}{*}{ (D) } & $3.83 \mathrm{~d}$ & 7 & $189 \pm 20$ \\
& & 8 & $153 \pm 20$ \\
\hline
\end{tabular}

Finally, the phase reconstructions using the CTF-based method are discussed. Figures 3.85 and 3.86 show reconstructions of the $B$. thuringiensis sample at final magnifications $M=369$ and $M=952$ sample using four and two different planes $z_{01}$, respectively. The visual impression of the images is quite good in comparison to the mHIO reconstructions of Figs. 3.80, 3.81. In particular, the noise in the data is significantly reduced which makes it a little easier for the eye to identify the cells and intracellular components such as endospores. However, the overall phase shift does not agree with the preceding iterative mHIO reconstructions due to a possibly non-optimal choice of regularisation parameters. An inspection of the PSDs suggests an overall achieved resolution of about $135 \mathrm{~nm}$ for the data of Fig. 3.85 and of about $100 \mathrm{~nm}$ for the data of Fig. 3.86. In comparison to the $\mathrm{mHIO}$ reconstructions the slightly improved resolution is in agreement to the 
applied doses of about $10^{5}$ Gy which are by about one order of magnitude higher for the case of the reconstruction scheme based on the CTF (cf. Tab. 3.14). The reconstruction of 3.86 may still be improved as here only data from two planes (instead of four) was available. The CTF-based reconstruction of region (A) of the B. subtilis sample of dried endospores is presented in Fig. 3.87a (final magnification $M=2222$ ). The image shows the three endospores of the mHIO reconstruction in Fig. 3.83a but with a clearly reduced noise level. Here, the PSD of the region with the two endospores (2) and (3) (Fig. 3.88c) indicates a resolution down to about $65 \mathrm{~nm}$ (half-period) which is in good agreement to the visual impression. The contrast of the phase map clearly allows to identify two regions within the spore (2) that may be linked to coat and the interior, namely cortex and core. These two structural regions of the endospore can be better distinguished in Figs. $3.87 \mathrm{~b}, 3.87 \mathrm{c}$ which show the horizontal and vertical gradient (filtered with $\sigma=5 \mathrm{px}$ gaussian). Size measurements from the reconstruction of Fig. 3.87a yield about $270 \mathrm{~nm}$ for the interior and $170-200 \mathrm{~nm}$ for the outer part (coat) along the horizontal direction. 

(A)

(C)

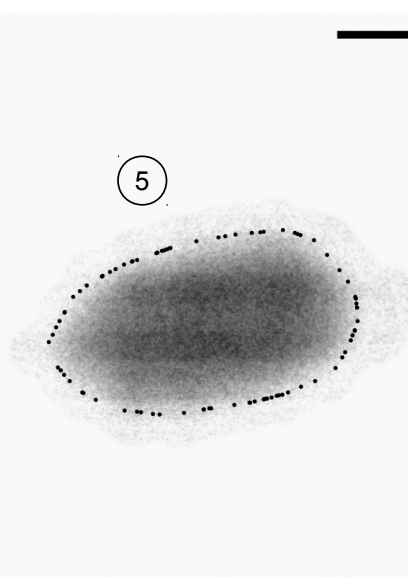

(c)
(B)

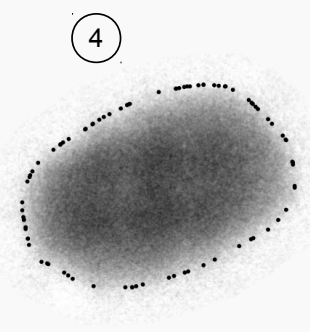

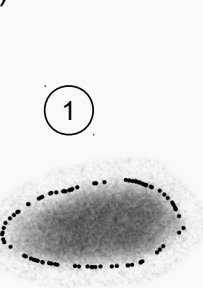

(a)

(3)
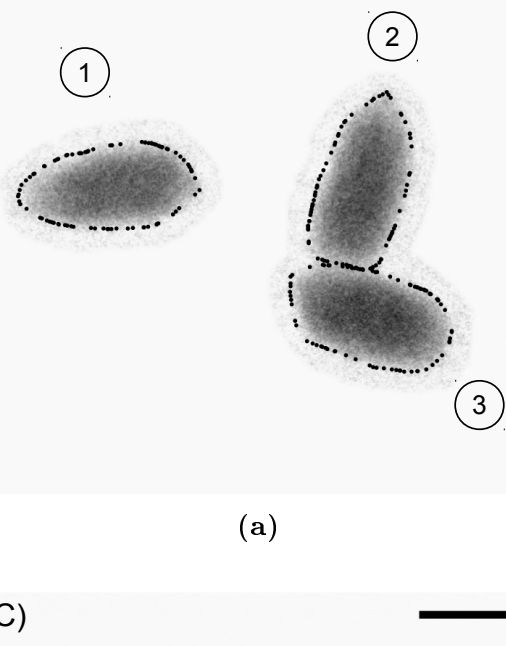

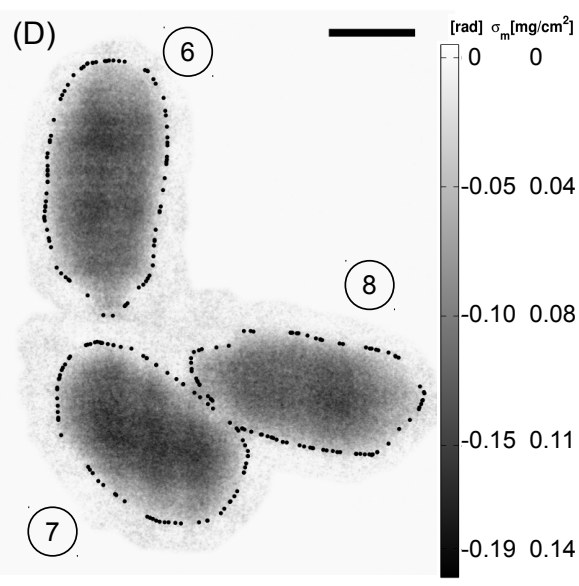

(d)

Figure 3.83: The figure presents phase reconstructions of endospores of B. subtilis of regions (A), (B), (C), (D) (cf. Fig. 3.76). The endospores are labelled from (1) to (8). The black dots indicate the boundaries used for mass estimations. The average mass per single endospore is $\left\langle m_{B . s u b .}\right\rangle=170 \pm 7 \mathrm{fg}$. The colorbar is the same in (a) (b) and (c) as in (d) The scale bars in (a), (b) (c) and (d) denote $1 \mu \mathrm{m}, 0.5 \mu \mathrm{m}$, $0.5 \mu \mathrm{m}$ and $0.5 \mu \mathrm{m}$, respectively. 


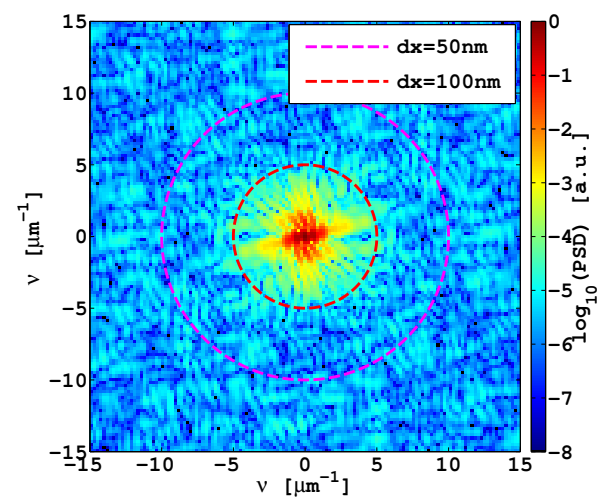

(a)

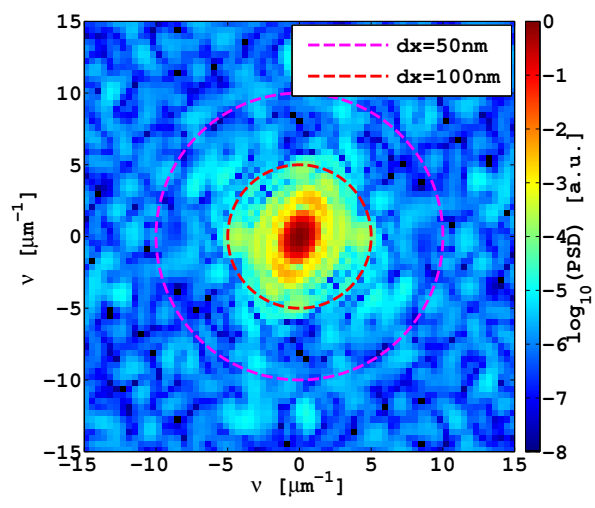

(b)

Figure 3.84: The figure presents calculated two-dimensional PSDs of the mHIO phase reconstructions of the B. subtilis data. (a) PSD of reconstruction of region (A) (Fig. 3.83a) and (b) PSD of reconstruction of region (B) (Fig. 3.83b) indicating resolutions in the range of $100 \mathrm{~nm}$. 


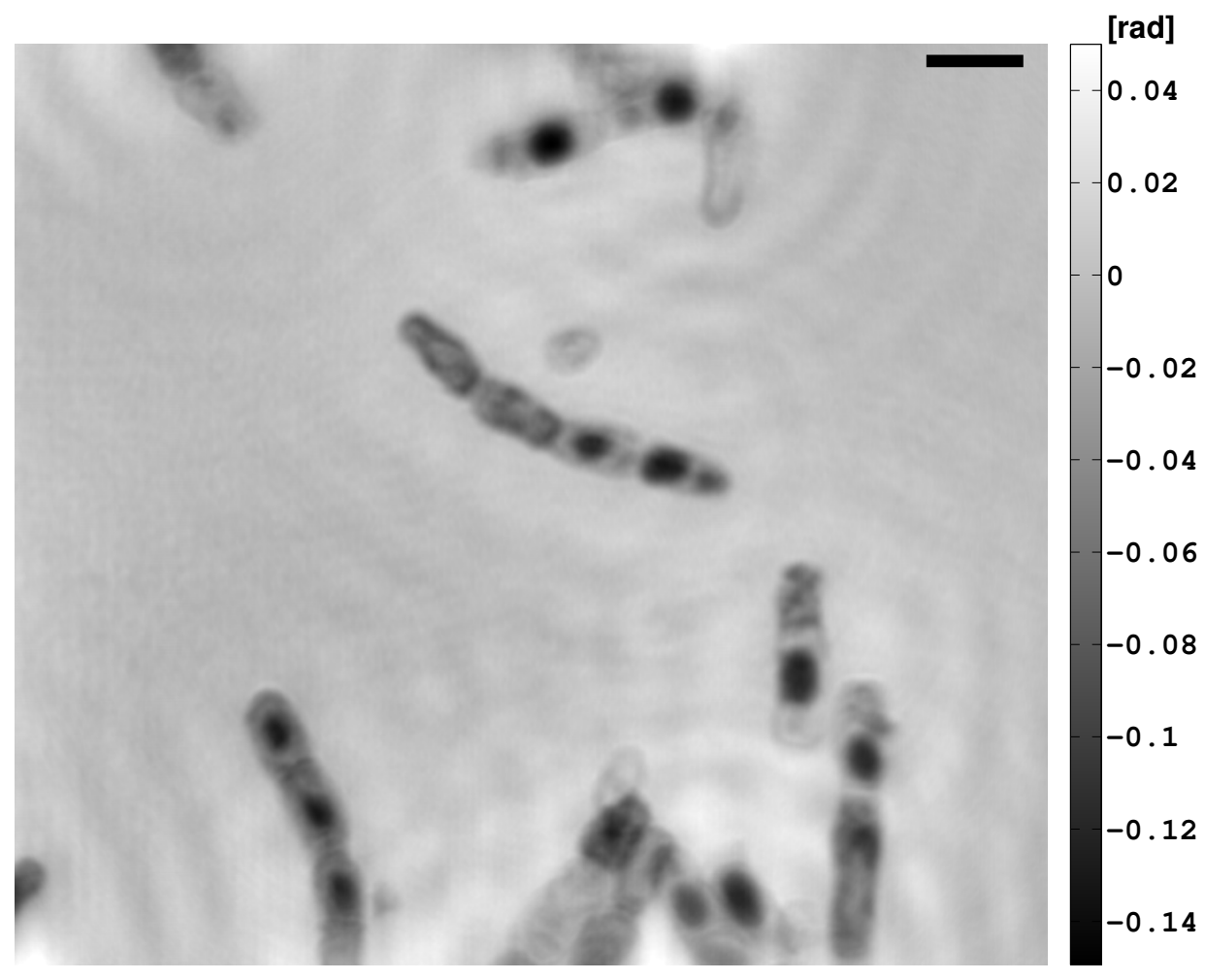

Figure 3.85: Region (B): The image shows a phase reconstruction using the CTF-based scheme of the $B$. thuringiensis sample. The scale bar denotes $2 \mu \mathrm{m}$. 


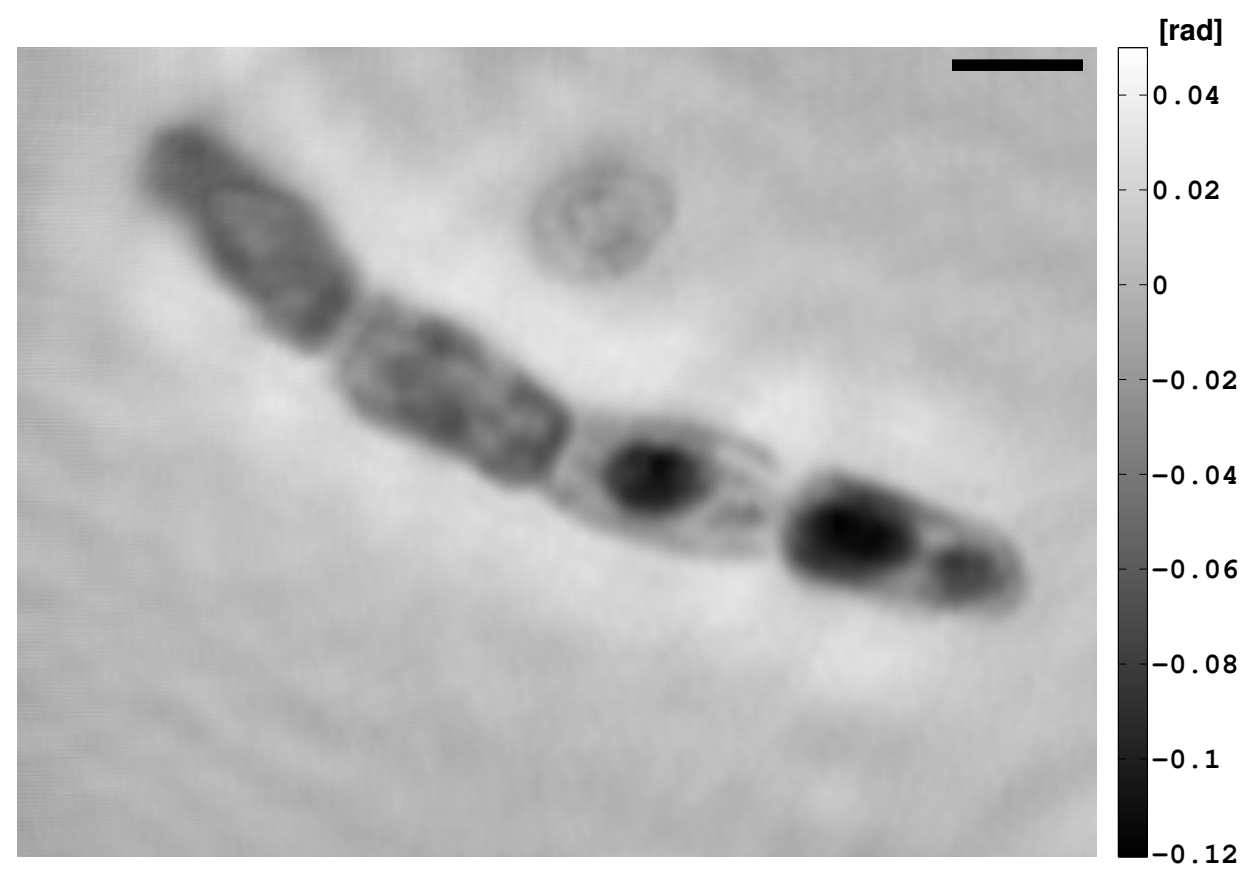

Figure 3.86: Region $(\mathrm{C})$ : The image shows a phase reconstruction using the CTF-based scheme of the $B$. thuringiensis sample. The scale bar denotes $1 \mu \mathrm{m}$. 


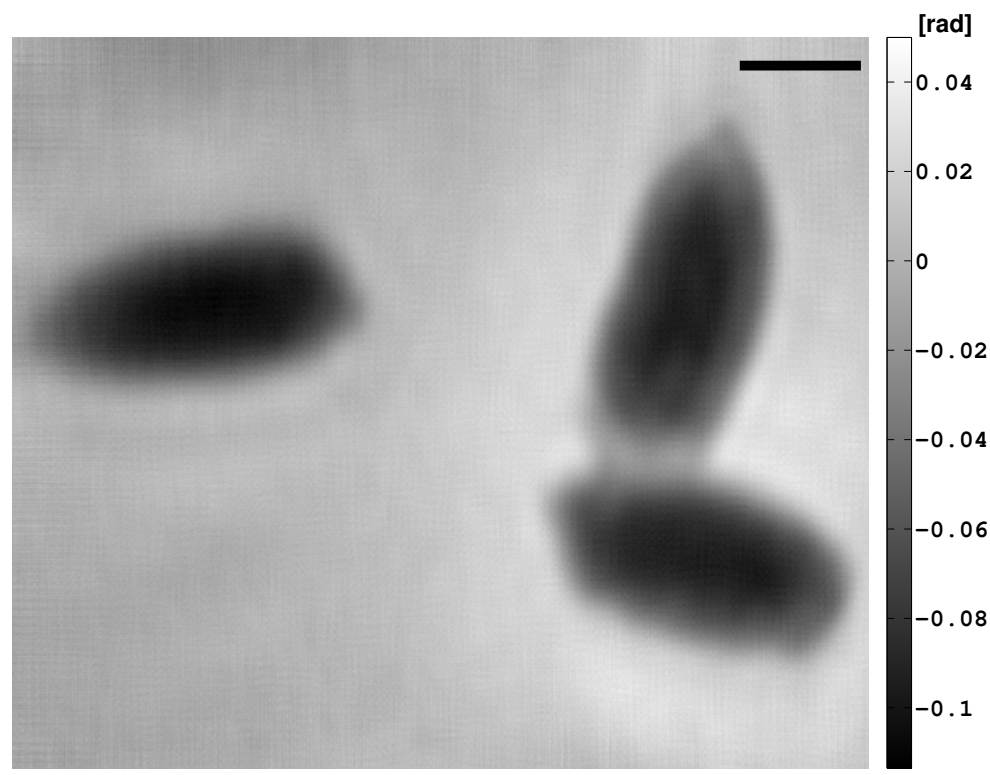

(a)

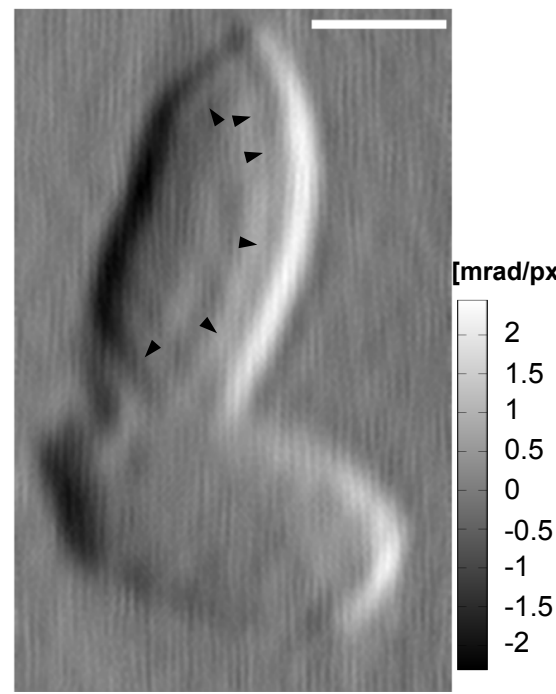

(b)

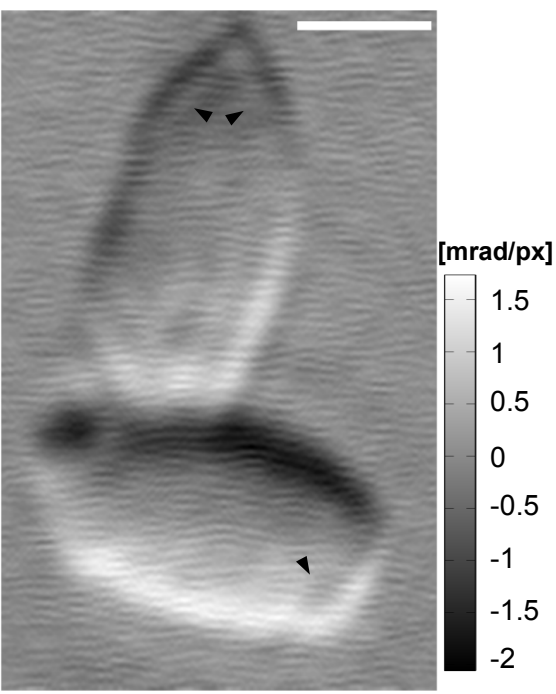

(c)

Figure 3.87: (a) shows the CTF-based reconstruction of region (A) of the B. subtilis sample. Three endospores can be seen. (b) (c) show calculated gradients (filtered with $\sigma=5 \mathrm{px}$ gaussian) in the horizontal and vertical direction of the region of two endospores of (a) The black arrow heads highlight the transition between two different structural regions of the endospore that are attributed to coat and inner part. The scale bars denotes $0.5 \mu \mathrm{m}$. 


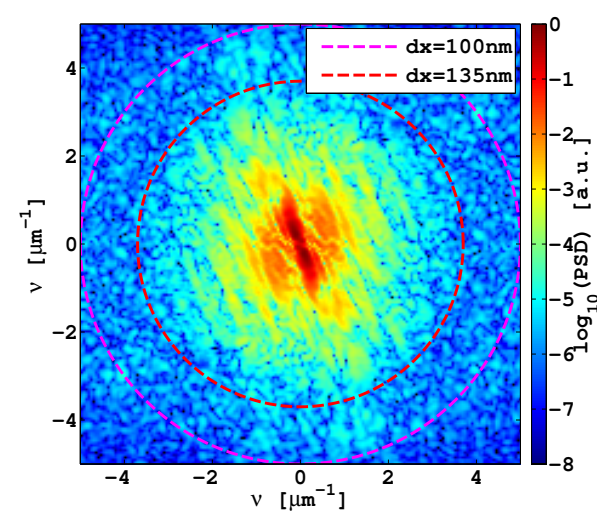

(a)

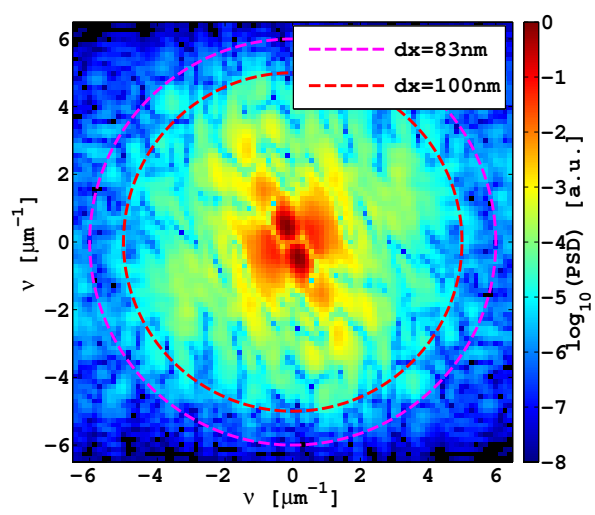

(b)

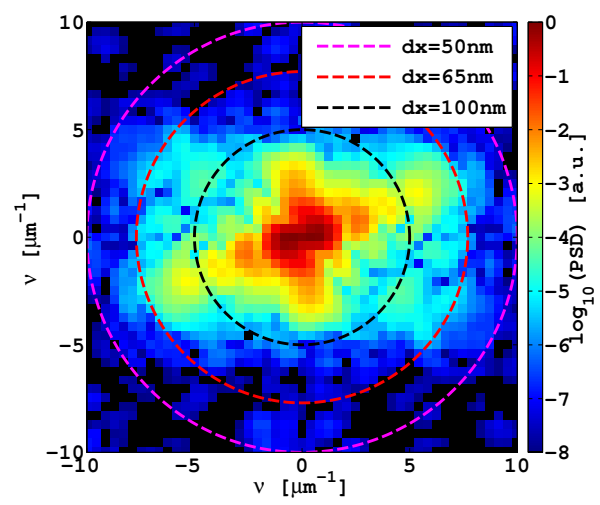

(c)

Figure 3.88: The figure presents calculated two-dimensional PSDs of the CTF-based phase reconstructions of both B. thuringiensis (regions (B) and (C)) and B. subtilis (region (A)) data. (a) (b) and (c) PSDs of reconstructions of Figs. 3.85, 3.86 and $3.87 \mathrm{a}$ indicating resolutions of about $135 \mathrm{~nm}, 100 \mathrm{~nm}$ and $60 \mathrm{~nm}$ (half-period). 


\subsubsection{Conclusion, Discussion \& Outlook}

In conclusion, two different waveguide-based X-ray imaging approaches have been successfully carried out on two samples of different endospore forming bacterial species, namely $B$. thuringiensis and B. subtilis. The versatility of the waveguide imaging scheme in terms of magnification allowed for obtaining either a large field of view for inspection of many bacterial cells at the same time or a small field of view that can be used for imaging single cells at high resolution. Here, the maximum resolution was achieved by using the CTF-based method yielding about $65 \mathrm{~nm}$ (half period) on single, unstained, unsliced and freeze-dried endospores of $B$. subtilis. In this case, structural changes from supposedly coat to cortex and core were observable. A mHIO reconstruction of single planes of the high magnification dataset used for CTF-based reconstructions was not carried out yet but is possible. In case of the $B$. thuringiensis data, the achieved resolution was lower but sufficient to differentiate different cellular components such as endospores. Indications of other possible intracellular occurrences such as BT-crystals were also found but necessitate further validation by either X-ray imaging data at higher resolution, a combination with tomography or nano-diffraction.

In addition, the iterative phase reconstructions using the mHIO algorithm could be used to determine the masses of entire, individual endospores of both bacterial species. Values in the range of $(113-192) \pm 20 \mathrm{fg}$ were obtained yielding average values for single endospores of $\left\langle m_{B . t h u}\right\rangle=153 \pm 9 \mathrm{fg}$ and $\left\langle m_{B . s u b .}\right\rangle=170 \pm 7 \mathrm{fg}$. These values can be compared with measurements of the wet weight, wet and dry density of endospores 38,304 . Carrera et al. used Percoll gradients to determine the mass density of comparably large amounts of endospores from $B$. thuringiensis 4055 and B. subtilis $1031 \mid 38$. From the determined density they calculated the mass of a single endospore by using an ellipsoid size model with size estimations from electron microscopy studies 37 . It should be noted, that the size estimation were obtained from chemically fixed and air-dried samples. They determined wet weights of $643 \mathrm{fg}$ (wet density $1.17 \mathrm{~g} / \mathrm{ml}$ ) and $196 \mathrm{fg}$ (wet density $1.22 \mathrm{~g} / \mathrm{ml}$, dry density $1.52 \mathrm{~g} / \mathrm{ml}$ ) for B. thuringiensis and B. subtilis, respectively. A dry density of $B$. thuringiensis endospores was not obtained in 38,304$]$. Therefore, a direct comparison cannot be made for $B$. thuringiensis endospores but it is noted that there is a comparably large gap between the wet weight of $643 \mathrm{fg}$ and the determined dry weight value of $\left\langle m_{\text {B.thu. }}\right\rangle=153 \pm 9 \mathrm{fg}$. The mass difference of $490 \mathrm{fg}$ would correspond to a volume of about $0.49 \mu \mathrm{m}^{3}$ of water $\left(V\left[\mu \mathrm{m}^{3}\right]=10^{-3} \mathrm{~m}[\mathrm{fg}] / \rho\left[\mathrm{g} / \mathrm{cm}^{3}\right]\right)$. However, if one applies the ellipsoid model $V=\pi L W^{2} / 6(L$ major and $W$ minor axis) 38 to the projections of the dried endospores here one obtains a similar volume. That is, the mass difference could be explained by the difference between the dried and wet state of the endospore but this conclusion seems to be unlikely as it would require the endospores to be filled with a comparably large amount of water. In case of the B. subtilis endospores, the comparison with the data from Carrera and co-workers is equally surprising. For instance, the volume estimations, that were used by Carrera et al. for determination of the wet weight, would yield a dry weight of $243 \mathrm{fg}$ for endospores of B. subtilis of dry den- 
sity $1.52 \mathrm{~g} / \mathrm{ml}$ which is close to the value found here. Taking also into account a reduced size of a dried endospore (in comparison to its wet state) would indicate that the dry weight must be even lower than $243 \mathrm{fg}$. However, the ellipsoid model can also be used to determine the dry density from the data here. This yields for the endospore $(2)$ with $L \approx 1.46 \mu \mathrm{m}$ and $W \approx 0.53 \mu \mathrm{m}$ a dry density of $\rho[\mathrm{g} / \mathrm{ml}]=10^{-3} \cdot \mathrm{m}[\mathrm{fg}] / V\left[\mu \mathrm{m}^{3}\right]=180 / 0.214=0.84$ which is significantly below the average spore density of $1.52 \mathrm{~g} / \mathrm{ml} \mid 38$. Hence, the conclusion is that in both cases the mass estimations appear to be suprisingly low. There are also other factors which influence the weight of the endospores. For instance, the differences likely arise from the comparison of endospores from two different B. subtilis strains. Moreover, it is known that the used sporulating medium has an influence on e.g. the coat of the endospore [38,67] (and references therein). Here, the preparation of the endospores differs from that in $[37,38 \mid$ by working without a special sporulating medium [67] and by using a different growth medium. Differences in size and density of the endospores are thus expected. As indicated above, the water content of the endospores affects the wet weight and the wet density and it is known that the endospore volume depends on its hydration level. According to Plomp and colleagues 232 (and references therein) endospores undergo swelling when exposed to water. Consequently, some volume may be filled with water and the weight thus increases. A systematic error may also be the reason for the obtained results here. However, the mHIO reconstructions from M. Bartels indicate that the obtained density values agree within a range of about $10 \%$ with the theoretical expectation as determined from measurements on an Au test sample 12 . In principle, the CTF-based phase maps are also quantitative $[45,47]$ but due to a possibly non-optimum choice of regularisation parameters small differences to the mHIO phase reconstructions were obtained. For this reason, the phase maps have not been converted to projected mass density maps yet.

In the future, more experimental data is desired in order to be better able to compare the results with other works. In particular, imaging of hydrated specimens appears to be appealing. Increasing the resolution and a combination with tomography may require preparation of frozen-hydrated samples but could allow determination of the water content of the endospores and its core which is not easily accessible otherwise. Moreover, a combination with cellular nano-diffraction may allow for a clear identification of BT-crystals. The ability to study microorganisms on the level of single cells can be of utmost importance when e.g. virulent species are investigated. 


\section{Summary, Conclusion \& Outlook}

A central goal of this thesis was an improvement of the ptychographic imaging capabilities of the Göttingen Instrument for Nano-Imaging with X-rays (GINIX) at the P10 beamline of the PETRA III light source with a possible application to microbial specimens on the level of single cells. Early experiments at the beginning of this thesis showed that the ptychographic imaging options at the GINIX were very limited. The experimentally achieved resolution of metallic test samples and biological specimens (not presented in this work) was considered to be limited by the used flux. More precisely, only a small fraction of about $10^{6}$ phot $/ \mathrm{s}$ of the fully available coherent photon flux of about $10^{11} \mathrm{phot} / \mathrm{s}$, as was determined in 258 , could be used due to the limited dynamic range of current photon counting detectors (cf. [98, 332]). The introduction of a Semi-Transparent Central Stop in front of the detector in order to attenuate the direct beam was carried out experimentally during two distinct experiments with successively decreasing Xray wavelength at the GINIX. The experiments and the analysis of the obtained data clearly shows that the introduction of an STCS can be made compatible with the ptychographic imaging scheme. The analysis of the first data (section 3.3 p. 132 ) revealed that a single attenuation factor can yield very good, quantitative reconstructions for the case of a uniform metal foil. Optimum results were obtained by comparing different reconstructions with a reference dataset. Another STCS was prepared of $\mathrm{Ge}$ for an experiment at $13.8 \mathrm{keV}$ (section 3.4, p. 144). Here, an optimum average transmission of about $2 \cdot 10^{-2}$ was found to be sufficient to attenuate the KB-imprint on the 'Lambda' detector to feasible levels, irrespective of the used slit settings. As an alternative, the measured attenuation profile was used successfully for the ptychographic reconstruction. The maximum achieved flux density of $10^{5} \mathrm{phot} / \mathrm{nm}^{2} / \mathrm{s}$ corresponds to a maximum photon flux of nearly $10^{10}$ phot/s being close to the optimum that is currently achievable at the GINIX and outstanding with respect to experimental achievements by other groups. The increase in flux could be used to obtain a significant increase in resolution for both experiments. In case of the data of the X-ray resolution chart (section 3.3, p. 132), the maximum resolution was determined to be close to $7 \mathrm{~nm}$ (half period) at parts of the object. This achieved resolution is also higher in comparison to the results that are presented in section 3.1 (cSAXS beamline, Swiss Light Source) and it is close to current achievements from other groups |266 287. On the other hand, the establishment of quantitative phase reconstructions on test samples represents a prerequisite for quantitative high-flux imaging of biological specimens and other samples. Moreover, ptychographic reconstructions of the KB mirror X-ray wavefield were obtained for different settings of the beam defining slits providing a good overview of the influence on the KB-focus. Earlier, a pinhole limiting the beam size in the focal plane of the KB mirrors was assigned to be essential for ptychographic reconstructions at the GINIX $\mid 98$. Here, a successful experiment was presented without the use of a pinhole.

Another central result of this thesis is the multi-modal ptychographic imaging experiment on the bacterial cells D. radiodurans (section 3.1, p. 69). Firstly, it 
represents the prototype of a scanning (coherent) SAXS experiment on the level of single cells that was also applied in recent works such as [105 240,323]. Presently, the obtained resolution in the range of about $2 \mathrm{~nm}^{-1}$ in reciprocal space ${ }^{26}$ exceeds any achieved real-space resolution on unstained cellular samples. For example, the achieved resolution of plane wave CDI experiments on single cells was reported to be in the range of $13-60 \mathrm{~nm} \mid 148,211,274]$. Taking also into account that the achievable resolution of biological cells using standard CDI methods is considered to be limited at $10 \mathrm{~nm}$ [141], it becomes clear that analyses on structures with a size of $2-6 \mathrm{~nm}$, being for example the relevant length scale of the ordering of the DNA in the bacterial nucleoid of $D$. radiodurans cells $\mid 77]$, should be carried out in reciprocal space until 'diffraction before destruction' methods 40 have been fully developed. In addition, the outlined combination of scanning SAXS with ptychography emphasizes the point that a sensible analysis in reciprocal space can benefit from a maximum accompanying resolution in real space. In particular, this becomes relevant when the local ordering that can be detected in reciprocal space changes on length scales of the beam size. On top, this work includes the combination of ptychography with tomography which can also be viewed of as an extension and improvement of resolution of preceding two-dimensional results [97]. There are a few other preceding works combining ptychography with tomography. Those samples consist of a part of a mouse femur [61], $\mathrm{SiO}_{2}$ [59] and resin-embedded, stained and chemically fixed cells $[109]$. In contrast to those works, the results here have been obtained on unstained, unsliced, freeze-dried cells that have not been treated by any chemical fixatives. In other words, the result is a quantitative three-dimensional representation of $D$. radiodurans cells on the level of single cells. Despite of radiation damage, reasonable quantitative information about the electron density of electron dense regions within the cells could be obtained yielding a mass density of about $1.6 \mathrm{~g} / \mathrm{cm}^{3}$. A subsequent analysis indicates that the dense regions may be tentatively linked with the DNA and the bacterial nucleoid. Assuming hypothetically that multiple copies of the genome are part of these dense regions within the single cells one can deduce a mass of about $110 \pm 40 \mathrm{fg}$ for the smallest unit of these genome copies. According to 277 there are at least 2 copies within one cell and there may be up to 10 . Hence, the mass of a single genome may be close to $55 \mathrm{fg}$ for the analysed cells. Another three-dimensional representation of freeze-dried cells of $D$. radiodurans has been obtained by waveguide-imaging shortly afterwards by M. Batels $[14$. However, dense regions were not as clearly observed as in this and following works 12,13 .

In case of the experiments on samples with a geobiological background (section 3.2 p. 110 ), the following results could be obtained. Firstly, the combination of hard X-ray phase contrast with X-ray fluorescence was found to be suitable to shed light on the important process of biocalcification. In particular, elements such as $\mathrm{Zn}$ and $\mathrm{Br}$ were found on basal elements of the aragonitic skeleton of the coralline demosponge Astrosclera willeyana. The element $\mathrm{Zn}$ is part of the metalloenzyme carbonic anhydrase (CA) being involved in the process of biocalcification. Here,

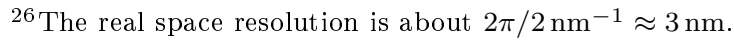


the $\mathrm{Zn}$ signal could be linked to the CAs of $A$. willeyana and the $\mathrm{Br}$ signal is indicative of the terminal stage of growth of the investigated $\mathrm{CaCO}_{3}$ spherulites. In addition, a quantitative scanning ptychography imaging experiment was successfully performed on a consortium of filamentous sulfur-oxidizing bacteria. From a methodical point of view, it was found to be very helpful to combine multiple ptychographic scans which allows for a reconstruction of a large field of view, quantitative phase map. Here, this yielded a phase map of about $20 \mu \mathrm{m} \times 40 \mu \mathrm{m}$ at a resolution of about $50 \mathrm{~nm}$.

The final experiments on unstained, unsliced and freeze-dried bacteria B. subtilis and $B$. thuringiensis (section 3.5, p. 161) further exemplify the potential of quantitative waveguide-imaging for microorganisms as pioneered in $12,14,94$. Using two distinct phase reconstruction techniques the two bacterial systems have been successfully imaged down to the level of single cells and endospores. Depending on the choice of reconstruction method, it was demonstrated that a resolution of about $65 \mathrm{~nm}$ (half period) can be obtained allowing for identifying different structural components of e.g. entire cells or endospores. In case of B. thuringiensis, endospores and possibly protein crystals (so called Bt-crystals) could be identified. The phase reconstructions of endospores of B. subtilis revealed internal details that can possibly be linked to core and cortex. In addition, the projected mass density and the mass of freeze-dried, entire individual endospores have been obtained for both bacterial species yielding average masses of $\left\langle m_{B . t h u}\right\rangle=153 \pm 9 \mathrm{fg}$ and $\left\langle m_{\text {B.sub. }}\right\rangle=170 \pm 7 \mathrm{fg}$. As shown here, the versatility of the waveguide-imaging scheme also allows for a quantitative inspection of a large field of view. Here, a covered area of about $100 \mu \mathrm{m} \times 50 \mu \mathrm{m}$ was achieved. The area can be further increased. That is, a large number of individual cells can be measured, too. The experiments also demonstrate that these bacteria are highly suitable sample systems for quantitative waveguide-imaging or other coherent diffractive imaging schemes such as ptychography.

In conclusion, the methodical improvements for ptychographic imaging at the GINIX pave the way for experiments with unprecedented flux. In principle, this includes quantitative investigations of biological and microbial samples on the level of single cells at a maximum resolution which likely necessitates a cryo-protected working condition. Recording of quantitative electron density (mass density) maps with a spatial resolution of a few nanometres should be routinely achievable in the future. The analysis of the influence of the beam defining slits on the ptychographic reconstructions (section 3.1 .8 , p. 94) showed that a non-optimum setting decreases the achievable resolution with standard reconstruction schemes. However, recent methodical progress with respect to ptychography under relaxed coherence conditions $[78,299]$ indicate that obtained results in this work may still be improved by using adapted algorithms. As a consequence, the dose-resolution efficiency which is especially important for imaging of biological specimens may be significantly improved. One promising experimental direction would be to use the high flux at the GINIX to significantly reduce scanning times for ptychographic imaging. In the future, experiments may also take great advantage of the possible combination of the ptychographic imaging capabilities at the GINIX with X-ray fluorescence 
and scanning (coherent) SAXS using a controlled KB beam size. Possible sample candidates for such experiments were addressed in this work. Scientific questions that initially led to choose them as possible test candidates for the imaging experiments turned out to be more challenging than expected. During the time of this thesis not only the quality of the X-ray imaging experiments could be raised but also the quality of sample preparation increased - not least due to the constant monitoring of the sample systems with electron microscopy. For instance, the question whether a local ordering can be observed in the nucleoid of the bacterium $D$. radiodurans towards the onset of the stationary phase by means of a signal in reciprocal space is still unanswered but may be reached in the near future. Other examples of experimental opportunities can be derived from the endospore forming bacteria. For instance, comparing quantitative three-dimensional density maps of the endospores in a dry and fully hydrated state may further elucidate their water content which is important for their heat resistance 283. Endospores may also be candidates for observing time snapshots of dynamical processes such as hydration or germination $[230,232]$ which are difficult to observe otherwise. 


\section{A Fourier Transform Terminology}

There are different definitions of the Fourier transform. Here, the Fourier transform of a well behaved function $f: \mathbb{R}^{2} \rightarrow \mathbb{C}$ is defined as 101:

$$
\mathcal{F}\{f\}(\vec{\nu}):=\iint_{-\infty}^{\infty} f(\vec{\rho}) \exp (-i 2 \pi \vec{\nu} \cdot \vec{\rho}) d \rho_{1} d \rho_{2} .
$$

The inverse Fourier transform of a well behaved function $\tilde{f}: \mathbb{R}^{2} \rightarrow \mathbb{C}$ is likewise defined:

$$
\mathcal{F}^{-1}\{\tilde{f}\}(\vec{\rho}):=\iint_{-\infty}^{\infty} \tilde{f}(\vec{\nu}) \exp (i 2 \pi \vec{\nu} \cdot \vec{\rho}) d \nu_{1} d \nu_{2}
$$

The requirement $\mathcal{F}^{-1}\{\mathcal{F}\{f\}\} \stackrel{!}{=} f(\vec{\rho})$ yields the Dirac $\delta$ function $^{27}$ :

$$
\delta\left(\vec{\rho}-\overrightarrow{\rho^{\prime}}\right)=\iint_{-\infty}^{\infty} \exp \left(-i 2 \pi \vec{\nu} \cdot\left(\vec{\rho}-\overrightarrow{\rho^{\prime}}\right)\right) d \nu_{1} d \nu_{2}
$$

Defining the convolution for two well behaved functions $f, g: \mathbb{R}^{2} \rightarrow \mathbb{C}$ with existing Fourier transforms $\tilde{f}(\vec{\nu})=\mathcal{F}\{f\}, \tilde{g}(\vec{\nu})=\mathcal{F}\{g\}$ as

$$
f \star g(\vec{\tau}):=\iint_{-\infty}^{\infty} f(\vec{\rho}) g(\vec{\tau}-\vec{\rho}) d \rho_{1} d \rho_{2},
$$

yields the 'convolution theorem' 101]:

$$
\mathcal{F}\{f \star g\}(\vec{\nu})=\tilde{f}(\vec{\nu}) \cdot \tilde{g}(\vec{\nu}),
$$

or

$$
\mathcal{F}\{f \cdot g\}(\vec{\nu})=\tilde{f} \star \tilde{g}(\vec{\nu}) .
$$

Likewise, defining the cross-correlation for two well behaved functions $f, g: \mathbb{R}^{2} \rightarrow$ $\mathbb{C}$ with existing Fourier transforms $\tilde{f}(\vec{\nu})=\mathcal{F}\{f\}, \tilde{g}(\vec{\nu})=\mathcal{F}\{g\}$ as

$$
f \circledast g(\vec{\tau}):=\iint_{-\infty}^{\infty} f(\vec{\rho}) g^{*}(\vec{\rho}-\vec{\tau}) d \rho_{1} d \rho_{2},
$$

one finds

$$
\mathcal{F}\{f \circledast g\}(\vec{\nu})=\tilde{f}(\vec{\nu}) \cdot \tilde{g}^{*}(\vec{\nu}) .
$$

In particular, choosing $g=f$ yields the 'autocorrelation theorem' 101:

$$
\mathcal{F}\{f \circledast f\}(\vec{\nu})=|\tilde{f}(\vec{\nu})|^{2} .
$$

Likewise, one obtains

$$
\mathcal{F}\left\{|f(\vec{\rho})|^{2}\right\}(\vec{\nu})=\tilde{f} \circledast \tilde{f}(\vec{\nu}) .
$$

\footnotetext{
${ }^{27}$ In fact, the Dirac $\delta$ function needs the theory of distributions cf. 58 .
} 


\section{B Culture Media}

Table B.1: Medium M53 Corynebacterium Agar of the DSMZ for cell culturing of $D$. radiodurans.

\begin{tabular}{lr}
\hline Casein peptone, tryptic digest & $10.0 \mathrm{~g}$ \\
Yeast extract & $5.0 \mathrm{~g}$ \\
Glucose & $5.0 \mathrm{~g}$ \\
$\mathrm{NaCl}$ & $5.0 \mathrm{~g}$ \\
$\mathrm{Agar}$ & $15.0 \mathrm{~g}$ \\
Distilled water & $1000.0 \mathrm{ml}$ \\
Adjust pH to & $7.2-7.4$. \\
\hline
\end{tabular}

Table B.2: LB medium (Lysogeny broth/ Luria-Bertani) for cell culturing of Bacillus spp. 119.

\begin{tabular}{lr}
\hline Tryptone & $10.0 \mathrm{~g}$ \\
Yeast extract & $5.0 \mathrm{~g}$ \\
$\mathrm{NaCl}$ & $10.0 \mathrm{~g}$ \\
Distilled water & $1000.0 \mathrm{ml}$ \\
\hline
\end{tabular}

\section{EM Protocols}

Table C.1: Dehydration series and resin embedding after M. Hoppert (cf. |140).

\begin{tabular}{lll} 
Medium & $\begin{array}{l}\text { Minimal Incubation } \\
\text { Time }[\mathrm{min}]\end{array}$ & $\begin{array}{l}\text { Incubation Temperature } \\
{\left[{ }^{\circ} \mathrm{C}\right]}\end{array}$ \\
\hline $\begin{array}{l}15 \%(\mathrm{w} / \mathrm{v}) \text { ethanol in } \\
\text { pure water }\end{array}$ & 15 & 0 \\
$30 \%(\mathrm{w} / \mathrm{v})$ ethanol & 30 & 0 \\
$50 \%(\mathrm{w} / \mathrm{v})$ ethanol & 30 & -20 \\
$70 \%(\mathrm{w} / \mathrm{v})$ ethanol & 30 & -20 \\
$95 \%(\mathrm{w} / \mathrm{v})$ ethanol & 30 & -20 \\
$97 \%(\mathrm{w} / \mathrm{v})$ ethanol & 15 & -20 \\
$99 \%(\mathrm{w} / \mathrm{v})$ ethanol & 15 & -20 \\
$100 \%(\mathrm{w} / \mathrm{v})$ ethanol & $2 \times 30$ & -20 \\
$33 . \overline{3} \%$ ethanol $66 . \overline{6} \%$ & 120 & room temperature \\
LR White & & \\
$100 \%$ LR White & 120 or over night & room temperature or 4 \\
& & if over night \\
\hline
\end{tabular}




\section{Discussion Missing Wedge}

Here, a short discussion of the effect of the missing wedge on the tomographic reconstruction of $D$. radiodurans cells (Fig. 3.43 p. 102) follows. It is known that the 'missing-wedge' problem of tomography causes anisotropic resolution [178]. In studies by electron tomography (ET) one usually has to deal with a 'missingwedge' of $\pm 20^{\circ}$. The 'missing-wedge' problem is thus more severe in ET than this study with a 'missing-wedge' of $\pm 13^{\circ}$. In addition, the three discarded projection angles $\alpha=47^{\circ}, 95^{\circ}, 141^{\circ}$ may also affect the quality of the tomographic reconstruction. To test this a comparison with ideal data, i.e. without noise, was made. A test phantom ('Shepp-Logan') with an extension comparable to the experimental situation was created (cf. Fig. D.1a). Tomographic reconstructions were calculated for the case of 90 projections with $\pm 0.5^{\circ}$ 'missing-wedge' (Fig. D.1b), 77 projections with $\pm 13^{\circ}$ 'missing-wedge' (Fig. D.1c) and 74 projections with $\pm 13^{\circ}$ 'missing-wedge' (three missing projections) (Fig. D.1d). As expected, best results are obtained in case of minimal 'missing-wedge'. To quantify the errors associated with missing data, the average was calculated over a certain area that is marked by white dots in Fig. D.1a and Fig. D.1d. A comparison yields a relative deviation of $\left\langle a_{\text {Fig. }}\right.$ D.1d $\rangle /\left\langle a_{\text {Fig. }}\right.$. D.1a $\rangle=0.91$, which is reasonable given the type of biophysical problem treated in this work and the fact that this is meant as a first proof of principle for 3D ptychographic reconstruction of cells. Furthermore, it is found that the three additional missing projections do not significantly increase the errors resulting already from the missing wedge (comp. Fig. D.1d and D.1c). Here it is stressed, that the $10 \%$ error is an upper limit, since the actual sample is flattened in the direction of the beam. 


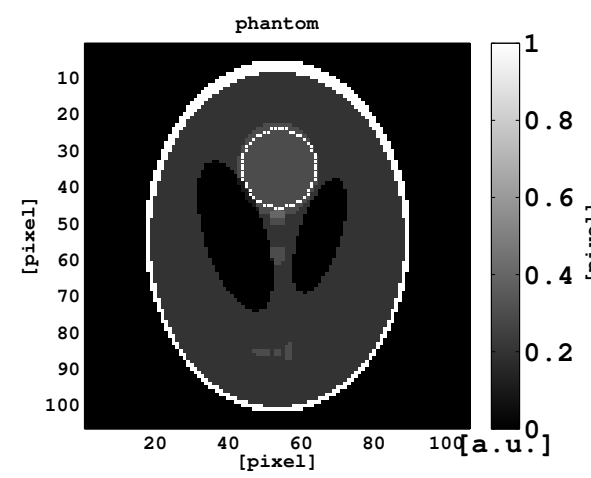

(a)

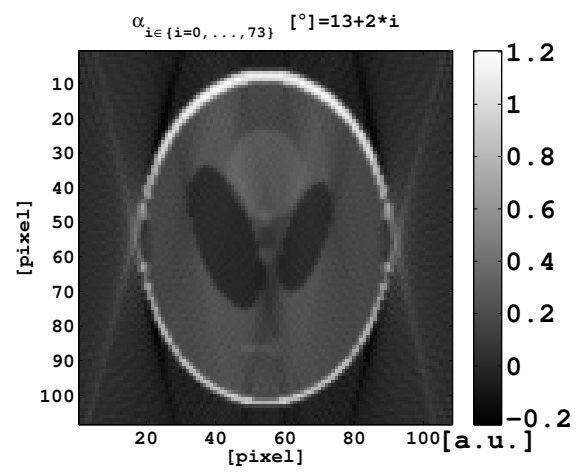

(c)

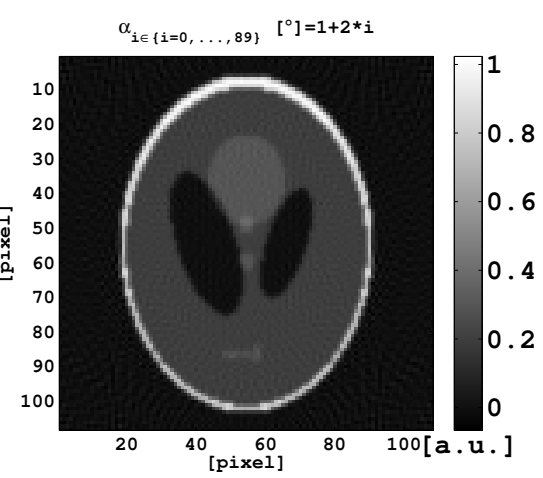

(b)

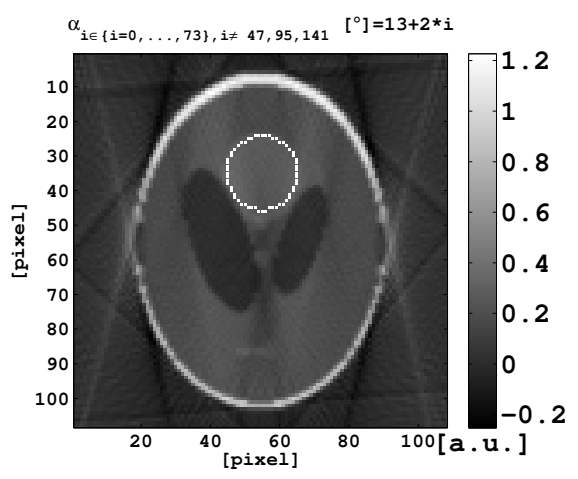

(d)

Figure D.1: ((a) 'Shepp-Logan' phantom used for simulating tomographic reconstructions. ((b) tomographic reconstruction for the case of 90 projections with $\pm 0.5^{\circ}$ 'missing-wedge', ((c)) tomographic reconstruction for the cases of 77 projections with $\pm 13^{\circ}$ 'missing-wedge' and ((d)) tomographic reconstruction for the cases of 74 projections with $\pm 13^{\circ}$ 'missing-wedge'. Dashed white dots in (a) and (d) label regions which have been averaged in order to compare the values with each other. 


\section{E ePIE Amplitude Reconstruction}

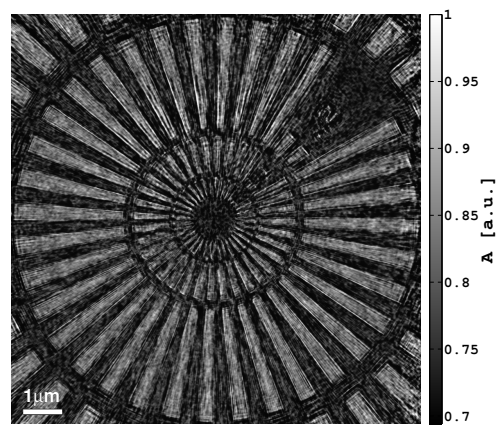

Figure E.1: The image presents the reconstructed amplitude corresponding to the ePIE reconstruction of the Siemens star resolution chart shown in Fig. 3.33 p. 85. 


\section{F TEM Images}

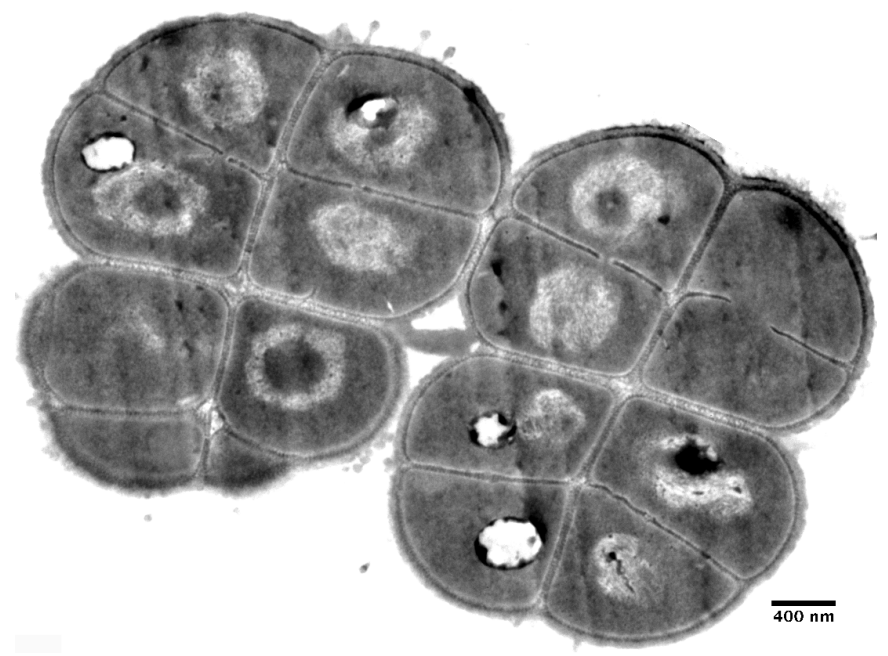

(a)

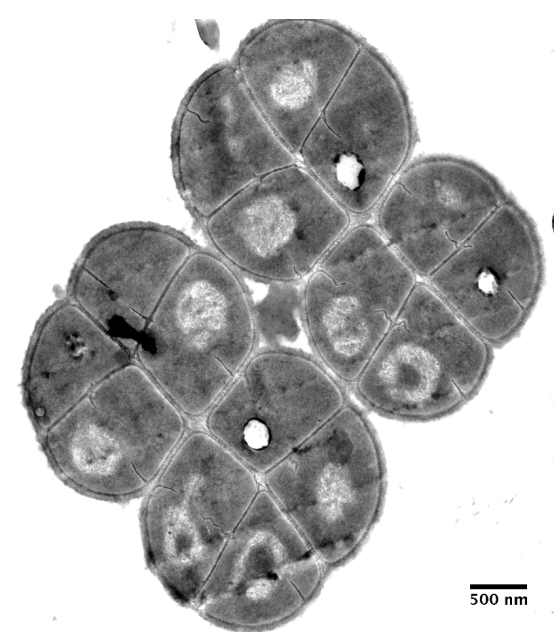

(b)

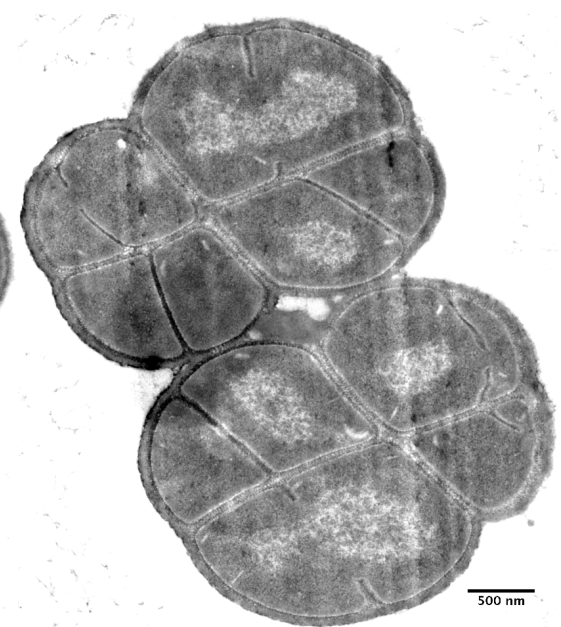

(c)

Figure F.1: The figure presents TEM images of ultra-thin sections of D. radiodurans cells that have been chemically fixed and stained (and fixed) with $\mathrm{OsO}_{4}$ prior to embedding and post-staining with uranyl acetate. Most of the cells seem to be in a state of cell division but the images also reveal artefacts of the preparation such as holes (white regions within the cells) and unbalanced staining (very dark regions within the cells). (b) also shows strong distortions from the knive along the cuttingdirection. 


\section{G STCS List}

Table G.1: Information about prepared STCSs are summarized below. The dimensions are indicated in the image below. The corresponding transmissions values are listed in table G.2

\begin{tabular}{lllllll}
\hline $\mathrm{E}$ & STCS & & \multicolumn{3}{l}{ STCS } \\
{$[\mathrm{keV}]$} & material & $\mathrm{a} \times \mathrm{b}\left[\mathrm{mm}^{2}\right]$ & $\mathrm{c}[\mu \mathrm{m}]$ & material & $\mathrm{a}^{\prime} \times \mathrm{b}^{\prime}\left[\mathrm{mm}^{2}\right]$ & $\mathrm{c}^{\prime}[\mu \mathrm{m}]$ \\
\hline 7.9 & $\mathrm{Si}$ & $8 \times 8$ & 200 & $\mathrm{Si}$ & $3 \times 3$ & 200 \\
7.9 & $\mathrm{Si}$ & $8 \times 8$ & 200 & $\mathrm{Si}$ & $4 \times 4$ & 200 \\
7.9 & $\mathrm{Si}$ & $16 \times 16$ & 200 & $\mathrm{Si}$ & $3 \times 3$ & 200 \\
7.9 & $\mathrm{Si}$ & $16 \times 16$ & 200 & $\mathrm{Si}$ & $5 \times 5$ & 200 \\
\hline $7.9 / 13.8$ & $\mathrm{Ge}$ & $4 \times 4$ & 100 & - & - & - \\
$7.9 / 13.8$ & $\mathrm{Ge}$ & $5 \times 5$ & 100 & - & - & - \\
\hline 13.8 & $\mathrm{Ag}$ & $16 \times 16$ & 35 & $\mathrm{Ag}$ & $5 \times 5$ & 75 \\
\hline
\end{tabular}

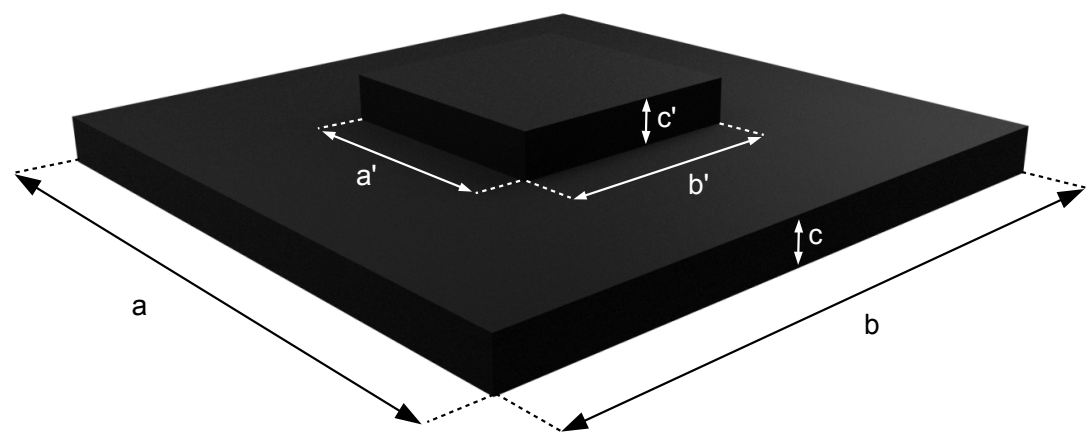


Table G.2: Calculated transmission values of different STCS materials, thicknesses and energies are listed below. Calculations were performed according to tabulated values [125].

\begin{tabular}{lllll}
\hline $\begin{array}{l}\text { STCS } \\
\text { material }\end{array}$ & $\begin{array}{l}\text { density } \\
{\left[\mathrm{g} / \mathrm{cm}^{3}\right]}\end{array}$ & $\begin{array}{l}\text { thickness } \\
{[\mu \mathrm{m}]}\end{array}$ & $\begin{array}{l}\text { energy } \\
{[\mathrm{keV}]}\end{array}$ & transmission \\
\hline $\mathrm{Si}$ & 2.363 & 200 & 7.9 & $5.04 \cdot 10^{-2}$ \\
$\mathrm{Si}$ & 2.363 & 400 & 7.9 & $2.54 \cdot 10^{-3}$ \\
$\mathrm{Ge}$ & 5.323 & 100 & 7.9 & $2.44 \cdot 10^{-2}$ \\
$\mathrm{Ge}$ & 5.323 & 100 & 13.8 & $2.88 \cdot 10^{-2}$ \\
$\mathrm{Ag}$ & 10.5 & 35 & 13.8 & $3.72 \cdot 10^{-1}$ \\
$\mathrm{Ag}$ & 10.5 & 110 & 13.8 & $4.46 \cdot 10^{-2}$ \\
& & & & \\
\hline
\end{tabular}

\section{H Algorithmic Parameters for Waveguide-Based Imaging}

The following tables list parameters of the reconstruction algorithm used in section 3.5 (p. 161).

Table H.1: Algorithmic parameters $\beta, \gamma, D$ of the mHIO algorithm (cf. section 1.3 .5 p. 39 ) used for reconstruction of the B. thuringiensis data are listed below. $n$ is the number of iterations that were carried out and $d_{n}$ is the error at the final iteration $n$.

\begin{tabular}{lllllll}
\hline Region & Figure (page) & $\beta$ & $\gamma$ & $D$ & $n$ & $d_{n}$ \\
\hline (A) & & 0.2 & 0.2 & 0.020 & 2000 & 0.036 \\
(A) & \multirow{2}{*}{$3.79(172)$} & 0.2 & 0.2 & 0.089 & 500 & 0.089 \\
(A) & & 0.2 & 0.2 & 0.064 & 500 & 0.064 \\
(A) & & 0.2 & 0.2 & 0.020 & 2000 & 0.105 \\
(B) & $3.80(174)$ & 0.2 & 0.2 & 0.0135 & 2000 & 0.015 \\
(C) & $3.81(\mathbf{1 7 5})$ & 0.2 & 0.2 & 0.046 & 2000 & 0.069 \\
\hline
\end{tabular}


Table H.2: $\quad$ Algorithmic parameters $\beta, \gamma, D$ of the mHIO algorithm (cf. section 1.3.5 p. 39) used for reconstruction of the $B$. subtilis data are listed below. $n$ is the number of iterations that were carried out and $d_{n}$ is the error at the final iteration $n$.

\begin{tabular}{|c|c|c|c|c|c|c|}
\hline Region & Figure (page) & $\beta$ & $\gamma$ & $D$ & $n$ & $d_{n}$ \\
\hline (A) & $3.83 a(179)$ & 0.2 & 0.2 & 0.095 & 2000 & 0.095 \\
\hline (B) & $3.83 \mathrm{~b}(\overline{179})$ & 0.2 & 0.2 & 0.041 & 2000 & 0.146 \\
\hline (C) & $3.83 \mathrm{c}(179)$ & 0.2 & 0.2 & 0.065 & 2000 & 0.064 \\
\hline (D) & $3.83 \mathrm{~d}(\overline{179})$ & 0.2 & 0.2 & 0.017 & 2000 & 0.017 \\
\hline
\end{tabular}

\section{References}

[1] Brian Abbey. From grain boundaries to single defects: A review of coherent methods for materials imaging in the x-ray sciences. JOM, 65(9):1183-1201, 2013.

[2] H Agaisse and D Lereclus. How does bacillus thuringiensis produce so much insecticidal crystal protein? J. Bacteriol., 177(21):6027-32, 1995.

[3] Saurabh (Rob) Aggarwal. What's fueling the biotech engine[mdash]2011 to 2012. Nat Biotech, 30(12):1191-1197, December 2012.

[4] Ashraf Al-Amoudi, Jiin-Ju Chang, Amélie Leforestier, Alasdair McDowall, Laurée Michel Salamin, Lars PO Norlén, Karsten Richter, Nathalie Sartori Blanc, Daniel Studer, and Jacques Dubochet. Cryo-electron microscopy of vitreous sections. The EMBO Journal, 23(18):3583-3588, 2004.

[5] Ashraf Al-Amoudi, Lars P.O. Norlen, and Jacques Dubochet. Cryo-electron microscopy of vitreous sections of native biological cells and tissues. J. Struct. Biol., 148(1):131-135, October 2004.

[6] J. Als-Nielsen and D. McMorrow. Elements of Modern X-ray Physics. 2nd edition edition, 2011.

[7] A.W. Anderson, H.C. Nordon, R.F. Cain, G. Parrish, and D. Dugan. Studies on a radio-resistant micrococcus. i. isolation, morphology, cultural characteristics and resistance to gamma radiation. Food Technol., 10:575, 1956.

[8] B J Ang and K W Nickerson. Purification of the protein crystal from bacillus thuringiensis by zonal gradient centrifugation. Appl. Environ. Microbiol., 36(4):625-626, 1978.

[9] A I Aronson, W Beckman, and P Dunn. Bacillus thuringiensis and related insect pathogens. Microbiol. Rev., 50(1):1-24, 1986.

[10] R Ballabriga, G Blaj, M Campbell, M Fiederle, D Greiffenberg, E H M Heijne, X Llopart, R Plackett, S Procz, L Tlustos, D Turecek, and 
W Wong. Characterization of the medipix3 pixel readout chip. J. Instrum., 6(01):C01052, 2011.

[11] R. Ballabriga, M. Campbell, E. Heijne, X. Llopart, L. Tlustos, and W. Wong. Medipix3: A 64-k pixel detector readout chip working in single photon counting mode with improved spectrometric performance. Nucl. Instrum. Methods Phys. Res., Sect. A, 633, Supplement 1(0):S15-S18, May 2011.

[12] Matthias Bartels. Cone-beam x-ray phase contrast tomography of biological samples Optimization of contrast, resolution and field of view. $\mathrm{PhD}$ thesis, Universität Göttingen, 2013.

[13] Matthias Bartels, Martin Krenkel, Johann Haber, Robin Wilke, and Tim Salditt. X-ray holographic imaging of hydrated biological cells in solution. submitted manuscript, 2014.

[14] Matthias Bartels, Marius Priebe, Robin Wilke, Sven Krüger, Klaus Giewekemeyer, Sebastian Kalbfleisch, Christian Olendrowitz, C, Michael Sprung, and Tim Salditt. Low-dose three-dimensional hard x-ray imaging of bacterial cells. Opt. Nanoscopy, 1(1), 2012.

[15] Darren J. Batey, Daniel Claus, and John M. Rodenburg. Information multiplexing in ptychography. Ultramicroscopy, 138(0):13-21, March 2014.

[16] James A. Baum and Thomas Malvar. Regulation of insecticidal crystal protein production in bacillus thuringiensis. Mol. Microbiol., 18(1):1-12, October 1995 .

[17] Heinz H. Bauschke, Patrick L. Combettes, and D. Russell Luke. Phase retrieval, error reduction algorithm, and Fienup variants: a view from convex optimization. J. Opt. Soc. Am. A, 19(7):1334-1345, 2002.

[18] Heinz H. Bauschke, Patrick L. Combettes, and D. Russell Luke. Hybrid projection-reflection method for phase retrieval. J. Opt. Soc. Am. A, 20(6):1025-1034, 2003.

[19] T C Beaman and P Gerhardt. Heat resistance of bacterial spores correlated with protoplast dehydration, mineralization, and thermal adaptation. Appl. Environ. Microbiol., 52(6):1242-1246, 1986.

[20] Mike Beckers, Tobias Senkbeil, Thomas Gorniak, Klaus Giewekemeyer, Tim Salditt, and Axel Rosenhahn. Drift correction in ptychographic diffractive imaging. Ultramicroscopy, 126(0):44-47, March 2013.

[21] Mike Beckers, Tobias Senkbeil, Thomas Gorniak, Michael Reese, Klaus Giewekemeyer, Sophie-Charlotte Gleber, Tim Salditt, and Axel Rosenhahn. Chemical Contrast in Soft X-Ray Ptychography. Phys. Rev. Lett., 107(20):208101, November 2011. 
[22] Karim Benzerara, Nicolas Menguy, Martin Obst, JarosÃÂaw Stolarski, Maciej Mazur, Tolek Tylisczak, Gordon E. Brown Jr., and Anders Meibom. Study of the crystallographic architecture of corals at the nanoscale by scanning transmission x-ray microscopy and transmission electron microscopy. Ultramicroscopy, 111(8):1268-1275, July 2011.

[23] Hans R. Bilger and Taufiq Habib. Knife-edge scanning of an astigmatic Gaussian beam. Appl. Opt., 24(5):686-690, March 1985.

[24] Max Born and Emil Wolf. Principles of optics: rth (expanded) edition. Principles of optics: 7th (expanded) edition by Born, M., 1999.

[25] Ilja N. Bronstein, Konstantin A. Semendjajew, G. Musiol, and H. Mühlig. Taschenbuch der Mathematik. Deutsch, Thun [u.a.], 2001.

[26] B. W. Brooks and R. G. E. Murray. Nomenclature for "micrococcus radiodurans" and other radiation-resistant cocci: Deinococcaceae fam. nov. and deinococcus gen. nov., including five species. International Journal of Systematic Bacteriology, 31(3):353-360, 1981.

[27] Peter Brüggeller and Erwin Mayer. Complete vitrification in pure liquid water and dilute aqueous solutions. Nature, 288(5791):569-571, December 1980.

[28] Yves V. Brun and Lawrence J. Shimkets, editors. Prokaryotic development. ASM Press, 2000.

[29] O Bunk, M Bech, T H Jensen, R Feidenhans'l, T Binderup, A Menzel, and F Pfeiffer. Multimodal x-ray scatter imaging. New Journal of Physics, 11(12):123016, 2009.

[30] Oliver Bunk, Martin Dierolf, Søren Kynde, Ian Johnson, Othmar Marti, and Franz Pfeiffer. Influence of the overlap parameter on the convergence of the ptychographical iterative engine. Ultramicroscopy, 108(5):481-487, April 2008.

[31] Grant Bunker. troduction to XAFS : a practical guide to X-ray absorption fine structure spectroscopy. Cambridge University Press, New York, 2010.

[32] Tilman Butz. Fouriertransformation für Fußgänger. Vieweg+Teubner Verlag / Springer Fachmedien Wiesbaden GmbH, Wiesbaden, 2012.

[33] Thorsten Buzug. Computed Tomography : From Photon Statistics to Modern Cone-Beam CT. Springer-Verlag, 2008.

[34] RJ Cano and MK Borucki. Revival and identification of bacterial spores in 25- to 40-million-year-old dominican amber. Science, 268(5213):1060-1064, May 1995. 
[35] S. Capaccioli and K. L. Ngai. Resolving the controversy on the glass transition temperature of water? The Journal of Chemical Physics, 135(10):104504, 2011.

[36] P.R. Carey, P. Fast, H. Kaplan, and M. Pozsgay. Molecular structure of the protein crystal from bacillus thuringiensis: a raman spectroscopic study. Biochim. Biophys. Acta, 872(3):169-176, August 1986.

[37] M. Carrera, R.O. Zandomeni, J. Fitzgibbon, and J.-L. Sagripanti. Difference between the spore sizes of bacillus anthracis and other bacillus species. $J$. Appl. Microbiol., 102(2):303-312, 2007.

[38] M. Carrera, R.O. Zandomeni, and J.-L. Sagripanti. Wet and dry density of bacillus anthracis and other bacillus species. J. Appl. Microbiol., 105(1):6877 , July 2008.

[39] Henry N. Chapman, Anton Barty, Stefano Marchesini, Aleksandr Noy, Stefan P. Hau-Riege, Congwu Cui, Malcolm R. Howells, Rachel Rosen, Haifeng He, John C. H. Spence, Uwe Weierstall, Tobias Beetz, Chris Jacobsen, and David Shapiro. High-resolution ab initio three-dimensional x-ray diffraction microscopy. J. Opt. Soc. Am. A, 23(5):1179-1200, 2006.

[40] Henry N. Chapman, Carl Caleman, and Nicusor Timneanu. Diffraction before destruction. Philos T Roy Soc B, 369(1647):20130313, 2014.

[41] Henry N. Chapman and Keith A. Nugent. Coherent lensless X-ray imaging. Nat Photon, 4(12):833-839, December 2010.

[42] Jesse N. Clark, Xiaojing Huang, Ross J. Harder, and Ian K. Robinson. Dynamic imaging using ptychography. Phys. Rev. Lett., 112(11):113901, March 2014.

[43] Jesse N. Clark, Corey T. Putkunz, Evan K. Curwood, David J. Vine, Robert Scholten, Ian McNulty, Keith A. Nugent, and Andrew G. Peele. Dynamic sample imaging in coherent diffractive imaging. Opt. Lett., 36(11):1954-1956, June 2011.

[44] D Claus, D J Robinson, D G Chetwynd, Y Shuo, W T Pike, José J De J Toriz Garcia, and J M Rodenburg. Dual wavelength optical metrology using ptychography. Journal of Optics, 15(3):035702, 2013.

[45] P. Cloetens, W. Ludwig, J. Baruchel, D. Van Dyck, J. Van Landuyt, J. P. Guigay, and M. Schlenker. Holotomography: Quantitative phase tomography with micrometer resolution using hard synchrotron radiation $\mathrm{x}$ rays. Appl. Phys. Lett., 75(19):2912-2914, 1999.

[46] Peter Cloetens. Contribution to Phase Contrast Imaging, Reconstruction and Tomography with Hard Synchrotron Radiation. PhD thesis, Vrije Universiteit Brussel, 1999. 
[47] Peter Cloetens, Régis Mache, Michel Schlenker, and Silva Lerbs-Mache. Quantitative phase tomography of Arabidopsis seeds reveals intercellular void network. PNAS, 103(39):14626-14630, 2006.

[48] L. R. Comolli, M. Kundmann, and K. H. Downing. Characterization of intact subcellular bodies in whole bacteria by cryo-electron tomography and spectroscopic imaging. J. Microsc., 223(1):40-52, 2006.

[49] James W. Cooley and John W. Tukey. An Algorithm for the Machine Calculation of Complex Fourier Series. Mathematics of Computation, 19(90):297$301,1965$.

[50] J. Cosmidis and K. Benzerara. Biomineralization Sourcebook: Characterization of Biominerals and Biomimetic Materials, chapter Soft x-ray scanning transmission spectromicroscopy, pages 115-134. CRC Press Taylor \& Francis Group, 2014.

[51] Michael M. Cox and John R. Battista. Deinococcus radiodurans - the consummate survivor. Nat Rev Micro, 3(11):882-892, November 2005.

[52] N. Crickmore, D. R. Zeigler, J. Feitelson, E. Schnepf, J. Van Rie, D. Lereclus, J. Baum, and D. H. Dean. Revision of the nomenclature for the bacillus thuringiensis pesticidal crystal proteins. Microbiol. Mol. Biol. Rev., 62(3):807-813, 1998.

[53] R. A. Crowther, D. J. DeRosier, and A. Klug. The reconstruction of a three-dimensional structure from projections and its application to electron microscopy. P. Roy. Soc. A-Math. Phy., 317(1530):319-340, 1970.

[54] C. Dahl and A. Prange. Inclusions in Prokaryotes: Microbial Monographs, chapter Bacterial sulfur globules: occurrence, structure and metabolism, pages 21-51. Berlin: Springer, 2006.

[55] M. J. Daly, E. K. Gaidamakova, V. Y. Matrosova, A. Vasilenko, M. Zhai, A. Venkateswaran, M. Hess, M. V. Omelchenko, H. M. Kostandarithes, K. S. Makarova, L. P. Wackett, J. K. Fredrickson, and D. Ghosal. Accumulation of $\mathrm{Mn}(\mathrm{II})$ in Deinococcus radiodurans Facilitates Gamma-Radiation Resistance. Science, 306(5698):1025-1028, November 2004.

[56] Michael J. Daly. A new perspective on radiation resistance based on Deinococcus radiodurans. Nat Rev Micro, 7(3):237-245, March 2009.

[57] Edward F. DeLong and Norman R. Pace. Environmental diversity of bacteria and archaea. Syst. Biol., 50(4):470-478, 2001.

[58] Philippe Dennery and Andreé Krzywicki. Mathematics for Physicists. Dover Publications, Inc. Mineola, New York, 1996. 
[59] Ana Diaz, Pavel Trtik, Manuel Guizar-Sicairos, Andreas Menzel, Pierre Thibault, and Oliver Bunk. Quantitative x-ray phase nanotomography. Phys. Rev. B, 85(2):020104, January 2012.

[60] M. Dierolf, I. Zanette, B. Enders, P. Thibault, A. Menzel, O. Bunk, and F. Pfeiffer. Oral presentation: 'Ptychographic reconstructions using shared data sets'. In Coherence 2012 "International Workshop on Phase Retrieval and Coherent Scattering", Fukuoka, Japan, 2012.

[61] Martin Dierolf, Andreas Menzel, Pierre Thibault, Philipp Schneider, Cameron M. Kewish, Roger Wepf, Oliver Bunk, and Franz Pfeiffer. Ptychographic X-ray computed tomography at the nanoscale. Nature, 467(7314):436-439, September 2010.

[62] Martin Dierolf, Pierre Thibault, Andreas Menzel, Cameron M. Kewish, Konstantins Jefimovs, Ilme Schlichting, Konstanze von König, Oliver Bunk, and Franz Pfeiffer. Ptychographic coherent diffractive imaging of weakly scattering specimens. NJP, 12(3):035017, 2010.

[63] Roberto Dinapoli, Anna Bergamaschi, Beat Henrich, Roland Horisberger, Ian Johnson, Aldo Mozzanica, Elmar Schmid, Bernd Schmitt, Akos Schreiber, Xintian Shi, and Gerd Theidel. Eiger: Next generation single photon counting detector for x-ray applications. Nucl. Instrum. Methods Phys. Res., 650(1):79-83, September 2011.

[64] Ray Dixon and Daniel Kahn. Genetic regulation of biological nitrogen fixation. Nat Rev Micro, 2(8):621-631, August 2004.

[65] F. Döring, A.L. Robisch, C. Eberl, M. Osterhoff, A. Ruhlandt, T. Liese, F. Schlenkrich, S. Hoffmann, M. Bartels, T. Salditt, and H.U. Krebs. Sub-5 $\mathrm{nm}$ hard x-ray point focusing by a combined kirkpatrick-baez mirror and multilayer zone plate. Opt. Express, 21(16):19311-19323, 2013.

[66] A. A. Driedger. The dna content of single cells of micrococcus radiodurans. Can. J. Microbiol., 16(11):1136-1137, November 1970.

[67] Adam Driks. Bacillus subtilis spore coat. Microbiol. Mol. Biol. Rev., 63(1):120, 1999.

[68] Adam Driks. The dynamic spore. PNAS, 100(6):3007-3009, 2003.

[69] Adam Driks and Peter Setlow. Prokaryotic development, chapter Morphogenesis And Properties Of The Bacterial Spore, pages 191-218. ASM Press, 2000 .

[70] Jacques Dubochet, Marc Adrian, Jiin-Ju Chang, Jean-Claude Homo, Jean Lepault, Alasdair W. McDowall, and Patrick Schultz. Cryo-Electron microscopy of vitrified specimens. Q. Rev. Biophys., 21(2):129-228, 1988. 
[71] T. B. Edo, D. J. Batey, A. M. Maiden, C. Rau, U. Wagner, Z. D. Pešić, T. A. Waigh, and J. M. Rodenburg. Sampling in x-ray ptychography. Phys. Rev. A, 87:053850, May 2013.

[72] Jonathan A. Eisen. The reca protein as a model molecule for molecular systematic studies of bacteria: Comparison of trees of recas and 16s rrnas from the same species. J. Mol. Evol., 41(6):1105-1123, 1995.

[73] Veit Elser. Phase retrieval by iterated projections. J. Opt. Soc. Am. A, 20(1):40-55, January 2003.

[74] Veit Elser and R. P. Millane. Reconstruction of an object from its symmetryaveraged diffraction pattern. Acta Crystallographica Section A, 64(2):273279, Mar 2008.

[75] M. Eltsov and J. Dubochet. Fine structure of the Deinococcus radiodurans nucleoid revealed by cryoelectron microscopy of vitreous sections. Journal Of Bacteriology, 187(23):8047-8054, 2005.

[76] M. Eltsov and J. Dubochet. Study of the Deinococcus radiodurans Nucleoid by Cryoelectron Microscopy of Vitreous Sections: Supplementary Comments. J. Bacteriol., 188(17):6053-6058, 2006.

[77] Mikhail Eltsov and Jacques Dubochet. Fine Structure of the Deinococcus radiodurans Nucleoid Revealed by Cryoelectron Microscopy of Vitreous Sections. J. Bacteriol., 187(23):8047-8054, 2005.

[78] B. Enders, M. Dierolf, P. Cloetens, M. Stockmar, F. Pfeiffer, and P. Thibault. Ptychography with broad-bandwidth radiation. APL, 104(17):171104, 2014.

[79] Jeff Errington. Regulation of endospore formation in bacillus subtilis. Nat Rev Micro, 1(2):117-126, November 2003.

[80] Stewart J. Fallon, Malcolm T. McCulloch, and Thomas P. Guilderson. Interpreting environmental signals from the coralline sponge astrosclera willeyana. Palaeogeogr. Palaeocl, 228:58-69, 2005.

[81] H. M. L. Faulkner and J. M. Rodenburg. Movable Aperture Lensless Transmission Microscopy: A Novel Phase Retrieval Algorithm. Phys. Rev. Lett., 93(2):023903, 2004.

[82] J. R. Fienup. Reconstruction of an object from the modulus of its Fourier transform. Opt. Lett., 3(1):27-29, 1978.

[83] J. R. Fienup. Phase retrieval algorithms: a comparison. Appl. Opt., 21(15):2758-2769, 1982.

[84] J. R. Fienup. Reconstruction of a complex-valued object from the modulus of its Fourier transform using a support constraint. J. Opt. Soc. Am. A, 4(1):118-123, 1987. 
[85] J. R. Fienup, T. R. Crimmins, and W. Holsztynski. Reconstruction of the support of an object from the support of its autocorrelation. J. Opt. Soc. Am., 72(5):610-624, May 1982.

[86] J. R. Fienup and C. C. Wackerman. Phase-retrieval stagnation problems and solutions. J. Opt. Soc. Am. A, 3(11):1897-1907, 1986.

[87] P. Fratzl. Small-angle scattering in materials science - a short review of applications in alloys, ceramics and composite materials. JApplCryst, 36(3 Part 1):397-404, 2003.

[88] P. Fratzl, H. F. Jakob, S. Rinnerthaler, P. Roschger, and K. Klaushofer. Position-Resolved Small-Angle X-ray Scattering of Complex Biological Materials. JApplCryst, 30(5 Part 2):765-769, 1997.

[89] C. Fuhse, C. Ollinger, and T. Salditt. Waveguide-Based Off-Axis Holography with Hard X-Rays. Phys. Rev. Lett., 97(25):254801, 2006.

[90] Christian Fuhse. X-ray waveguides and waveguide-based lensless imaging. $\mathrm{PhD}$ thesis, University of Göttingen, 2006.

[91] Christian Fuhse, Ansgar Jarre, Christoph Ollinger, Jens Seeger, Tim Salditt, and Remi Tucoulou. Front-coupling of a prefocused x-ray beam into a monomodal planar waveguide. Applied Physics Letters, 85(11):1907-1909, 2004.

[92] R. W. Gerchberg and W. O. Saxton. A Practical Algorithm for the Determination of Phase from Image and Diffraction Plane Pictures. Optik (Jena), 35:237-246, 1972.

[93] Tillman U Gerngross. Advances in the production of human therapeutic proteins in yeasts and filamentous fungi. Nat Biotech, 22(11):1409-1414, November 2004.

[94] K. Giewekemeyer, S. P. Krüger, S. Kalbfleisch, M. Bartels, C. Beta, and T. Salditt. X-ray propagation microscopy of biological cells using waveguides as a quasipoint source. Phys. Rev. A, 83(2):023804, Feb 2011.

[95] Klaus Giewekemeyer. A study on new approaches in coherent x-ray microscopy of biological specimens. PhD thesis, Universität Göttingen, 2011.

[96] Klaus Giewekemeyer, Hugh T. Philipp, Robin N. Wilke, Andrew Aquila, Markus Osterhoff, Mark W. Tate, Katherine S. Shanks, Alexey V. Zozulya, Tim Salditt, Sol M. Gruner, and Adrian P. Mancuso. High-dynamic-range coherent diffractive imaging: ptychography using the mixed-mode pixel array detector. J. Synchrotron Rad., 21(5):1167-1174, 2014. 
[97] Klaus Giewekemeyer, Pierre Thibault, Sebastian Kalbfleisch, André Beerlink, Cameron M. Kewish, Martin Dierolf, Franz Pfeiffer, and Tim Salditt. Quantitative biological imaging by ptychographic x-ray diffraction microscopy. PNAS, 107(2):529-534, 2010.

[98] Klaus Giewekemeyer, Robin N. Wilke, Markus Osterhoff, Matthias Bartels, Sebastian Kalbfleisch, and Tim Salditt. Versatility of a hard x-ray kirkpatrick-baez focus characterized by ptychography. J. Synchrotron Rad., 20(3):490-497, 2013.

[99] O. Glatter and O. Kratky, editors. Small angle X-ray scattering. Acad. Pr., London [u.a.], 1982.

[100] Sophie-Charlotte Gleber. Soft X-ray stereo microscopy for investigation of dynamics and elemental distribution of colloidal systems from the environment. PhD thesis, Universität Göttingen, 2009.

[101] Joseph W. Goodman. Introduction to Fourier Optics. Roberts \& Company: Englewood, Colorado, 2005.

[102] Sergey Gorelick, Vitaliy A Guzenko, Joan Vila-Comamala, and Christian David. Direct e-beam writing of dense and high aspect ratio nanostructures in thick layers of PMMA for electroplating. Nanotechnology, 21(29):295303, 2010.

[103] Sergey Gorelick, Joan Vila-Comamala, Vitaliy A. Guzenko, Ray Barrett, Murielle Salome, and Christian David. High-efficiency Fresnel zone plates for hard X-rays by $100 \mathrm{keV}$ e-beam lithography and electroplating. J. Synchrotron Rad., 18(3):442-446, 2011.

[104] Thomas Gorniak. Melanosomen im Real- und Fourierraum. PhD thesis, Universität Heidelberg, 2013.

[105] Thomas Gorniak, Tamas Haraszti, Vasyl M. Garamus, Andreas R. Buck, Tobias Senkbeil, Marius Priebe, Adam Hedberg-Buenz, Demelza Koehn, Tim Salditt, Michael Grunze, Michael G. Anderson, and Axel Rosenhahn. Nanoscale morphology of melanosomes revealed by small-angle x-ray scattering. PLoS ONE, 9(3):e90884, March 2014.

[106] Aurelien Gourrier, Wolfgang Wagermaier, Manfred Burghammer, Donna Lammie, Himadri S. Gupta, Peter Fratzl, Christian Riekel, Tim J. Wess, and Oskar Paris. Scanning X-ray imaging with small-angle scattering contrast. Journal of Applied Crystallography, 40(s1):s78-s82, 2007.

[107] André Guinier and Gérard Fournet. Small-angle scattering of x-rays. Wiley, New York, NY, 1955.

[108] Manuel Guizar-Sicairos. Methods for Coherent Lensless Imaging and X-ray Wavefront Measurement. PhD thesis, University of Rochester. Institute of Optics, 2010., 2010. 
[109] Manuel Guizar-Sicairos, Ana Diaz, Mirko Holler, Miriam S. Lucas, Andreas Menzel, Roger A. Wepf, and Oliver Bunk. Phase tomography from x-ray coherent diffractive imaging projections. Opt. Express, 19(22):21345-21357, October 2011.

[110] Manuel Guizar-Sicairos and James R. Fienup. Phase retrieval with transverse translation diversity: a nonlinearoptimization approach. Opt. Express, 16(10):7264-7278, May 2008.

[111] Manuel Guizar-Sicairos, Mirko Holler, Ana Diaz, Joan Vila-Comamala, Oliver Bunk, and Andreas Menzel. Role of the illumination spatial-frequency spectrum for ptychography. Phys. Rev. B, 86(10):100103, September 2012.

[112] Manuel Guizar-Sicairos, Ian Johnson, Ana Diaz, Mirko Holler, Petri Karvinen, Hans-Christian Stadler, Roberto Dinapoli, Oliver Bunk, and Andreas Menzel. High-throughput ptychography using eiger-scanning x-ray nanoimaging of extended regions. Opt. Express, 22(12):14859-14870, 2014.

[113] Manuel Guizar-Sicairos, Samuel T. Thurman, and James R. Fienup. Efficient subpixel image registration algorithms. Opt. Lett., 33(2):156-158, January 2008.

[114] Johann Haber. Optimization of the wafer bondig process for the fabrication of lithographic x-ray waveguides. Master's thesis, Universität Göttingen, 2013.

[115] J. Hagemann, A.-L. Robisch, D. R. Luke, C. Homann, T. Hohage, P. Cloetens, H. Suhonen, and T. Salditt. Reconstruction of wave front and object for inline holography from a set of detection planes. Opt. Express, 22(10):11552-11569, May 2014.

[116] C. L. Hannay and P. Fitz-James. The protein crystals of bacillus thuringiensis berliner. Can. J. Microbiol., 1(8):694-710, October 1955.

[117] M T Hansen. Multiplicity of genome equivalents in the radiation-resistant bacterium Micrococcus radiodurans. Journal of Bacteriology, 134(1):71-75, April 1978.

[118] Harsojo, Shigeru Kitayama, and Akira Matsuyama. Genome multiplicity and radiation resistance in micrococcus radiodurans. J. Biochem., 90(3):877-880, 1981.

[119] Colin R. Harwood and Simon M. Cutting, editors. Molecular biological methods for Bacillus. Wiley, 1990.

[120] Yi He. High cell density production of deinococcus radiodurans under optimized conditions. Journal of Industrial Microbiology $\&$ Biotechnology, 36(4):539-546, 2009. 
[121] Ian M. Head, D. Martin Jones, and Wilfred F. M. Roling. Marine microorganisms make a meal of oil. Nat Rev Micro, 4(3):173-182, March 2006.

[122] R. Hegerl and W. Hoppe. Dynamische theorie der kristallstrukturanalyse durch elektronenbeugung im inhomogenen primärstrahlwellenfeld. Berichte der Bunsengesellschaft für physikalische Chemie, 74(11):1148-1154, November 1970 .

[123] R. Hegerl and W. Hoppe. Influence of electron noise on three-dimensional image reconstruction. Z. Naturforsch., 31a:1717-1721, 1976.

[124] B. L. Henke. Low energy x-ray interactions: Photoionization, scattering, specular and bragg reflection. AIP Conference Proceedings, 75(1):146-155, 1981.

[125] B. L. Henke, E. M. Gullikson, and J. C. Davis. X-Ray Interactions: Photoabsorption, Scattering, Transmission, and Reflection at $\mathrm{E}=50-30,000 \mathrm{eV}$, $\mathrm{Z}=1$ 1-92. Atomic Data and Nuclear Data Tables, 54(2):181-342, July 1993.

[126] Reinhard Hensel, Wilhelm Demharter, Otto Kandler, Reiner M. Kroppenstedt, and Erko Stackebrandt. Chemotaxonomic and molecular-genetic studies of the genus thermus: Evidence for a phylogenetic relationship of thermus aquaticus and thermus ruber to the genus deinococcus. International Journal of Systematic Bacteriology, 36(3):444-453, 1986.

[127] TA Hentunen, PL Harkonen, and HK Vaananen. The Carbonic Anhydrases: New Horizons, volume 90, chapter Carbonic anhydrases in calcified tissues, pages 491-497. Basel, Switzerland: Birkhäuser Verlag, 2000.

[128] R. Hesse and D. Luke. Nonconvex notions of regularity and convergence of fundamental algorithms for feasibility problems. SIAM J. Optim., 23(4):2397-2419, January 2013.

[129] Robert Hesse. Fixed Point Algorithms for Nonconvex Feasibility with Applications. PhD thesis, Universität Göttingen, 2014.

[130] O. Hignette, P. Cloetens, G. Rostaing, P. Bernard, and C. Morawe. Efficient sub $100 \mathrm{~nm}$ focusing of hard x rays. Review of Scientific Instruments, $76(6): 063709,2005$.

[131] AP Hitchcock, T Tyliszczak, M Obst, G Swerhone, and J Lawrence. Improving sensitivity in soft x-ray stxm using low energy x-ray fluorescence. Microscopy and Microanalysis, 16(Supplement S2):924-925, 2010.

[132] H Höfte and H R Whiteley. Insecticidal crystal proteins of bacillus thuringiensis. Microbiol. Rev., 53(2):242-255, 1989. 
[133] M. Holler, A. Diaz, M. Guizar-Sicairos, P. Karvinen, Elina Färm, Emma Härkönen, Mikko Ritala, A. Menzel, J. Raabe, and O. Bunk. X-ray ptychographic computed tomography at $16 \mathrm{~nm}$ isotropic $3 \mathrm{~d}$ resolution. Sci. Rep., $4: 3857,2014$.

[134] Susanne Hönig, Robert Hoppe, Jens Patommel, Andreas Schropp, Sandra Stephan, Sebastian Schöder, Manfred Burghammer, and Christian G. Schroer. Full optical characterization of coherent x-ray nanobeams by ptychographic imaging. Opt. Express, 19(17):16324-16329, August 2011.

[135] R. Hoppe, J. Reinhardt, G. Hofmann, J. Patommel, J.-D. Grunwaldt, C. D. Damsgaard, G. Wellenreuther, G. Falkenberg, and C. G. Schroer. Highresolution chemical imaging of gold nanoparticles using hard x-ray ptychography. Appl. Phys. Lett., 102(20):203104, May 2013.

[136] W. Hoppe. Beugung im inhomogenen Primarstrahlwellenfeld. I. Prinzip einer Phasenmessung von Elektronenbeungungsinterferenzen. Acta Crystallographica Section A, 25(4):495-501, 1969.

[137] W. Hoppe. Beugung im inhomogenen primarstrahlwellenfeld. i. prinzip einer phasenmessung von elektronenbeungungsinterferenzen. Acta Crystallographica Section A, 25(4):495-501, 1969.

[138] W. Hoppe. Beugung im inhomogenen primarstrahlwellenfeld. iii. amplituden- und phasenbestimmung bei unperiodischen objekten. Acta Crystallographica Section A, 25(4):508-514, 1969.

[139] W. Hoppe and G. Strube. Beugung in inhomogenen primarstrahlenwellenfeld. ii. lichtoptische analogieversuche zur phasenmessung von gitterinterferenzen. Acta Crystallographica Section A, 25(4):502-507, 1969.

[140] Michael Hoppert and Andreas Holzenburg. Electron microscopy in microbiology. BIOS Scientific, 1998.

[141] M.R. Howells, T. Beetz, H.N. Chapman, C. Cui, J.M. Holton, C.J. Jacobsen, J. Kirz, E. Lima, S. Marchesini, H. Miao, D. Sayre, D.A. Shapiro, J.C.H. Spence, and D. Starodub. An assessment of the resolution limitation due to radiation-damage in X-ray diffraction microscopy. Journal of Electron Spectroscopy and Related Phenomena, 170(1-3):4-12, March 2009.

[142] Kristin Høydalsvik, Jostein Bø Fløystad, Tiejun Zhao, Morteza Esmaeili, Ana Diaz, Jens W. Andreasen, Ragnvald H. Mathiesen, Magnus Rønning, and Dag W. Breiby. In situ x-ray ptychography imaging of high-temperature $\mathrm{CO}_{2}$ acceptor particle agglomerates. Appl. Phys. Lett., 104(24):241909, 2014.

[143] Xiaojing Huang, Michael Wojcik, Nicolas Burdet, Isaac Peterson, Graeme R. Morrison, David J. Vine, Daniel Legnini, Ross Harder, Yong S. Chu, and Ian K. Robinson. Quantitative x-ray wavefront measurements of fresnel zone 
plate and k-b mirrors using phase retrieval. Opt. Express, 20(21):2403824048, October 2012.

[144] Xiaojing Huang, Hanfei Yan, Evgeny Nazaretski, Raymond Conley, Nathalie Bouet, Juan Zhou, Kenneth Lauer, Li Li, Daejin Eom, Daniel Legnini, Ross Harder, Ian K. Robinson, and Yong S. Chu. $11 \mathrm{~nm}$ hard x-ray focus from a large-aperture multilayer laue lens. Sci. Rep., 3:3562, December 2013.

[145] Daniel J. Jackson, Luciana Macis, Joachim Reitner, Bernard M. Degnan, and Gert Worheide. Sponge Paleogenomics Reveals an Ancient Role for Carbonic Anhydrase in Skeletogenesis. Science, 316(5833):1893-1895, 2007.

[146] John David Jackson. Classical electrodynamics. Wiley, 1999.

[147] A. Jarre, C. Fuhse, C. Ollinger, J. Seeger, R. Tucoulou, and T. Salditt. Two-Dimensional Hard X-Ray Beam Compression by Combined Focusing and Waveguide Optics. Phys. Rev. Lett., 94(7):074801, Feb 2005.

[148] Huaidong Jiang, Changyong Song, Chien-Chun Chen, Rui Xu, Kevin S. Raines, Benjamin P. Fahimian, Chien-Hung Lu, Ting-Kuo Lee, Akio Nakashima, Jun Urano, Tetsuya Ishikawa, Fuyuhiko Tamanoi, and Jianwei Miao. Quantitative 3D imaging of whole, unstained cells by using X-ray diffraction microscopy. PNAS, 107(25):11234-11239, 2010.

[149] I. Johnson, A. Bergamaschi, J. Buitenhuis, R. Dinapoli, D. Greiffenberg, B. Henrich, T. Ikonen, G. Meier, A. Menzel, A. Mozzanica, V. Radicci, D. K. Satapathy, B. Schmitt, and X. Shi. Capturing dynamics with eiger, a fast-framing x-ray detector. J. Synchrotron Rad., 19(6):1001-1005, 2012.

[150] Frank E. Jones. Evaporation of Water: With Emphasis on Applications and Measurements. Lewis Publisher, Inc., 1992.

[151] Avinash C. Kak and Malcolm Slaney. Principles of computerized tomographic imaging. IEEE Press, New York, 1988.

[152] S. Kalbfleisch, H. Neubauer, S. P. Krüger, M. Bartels, M. Osterhoff, D. D. Mai, K. Giewekemeyer, B. Hartmann, M. Sprung, and T. Salditt. The Göttingen Holography Endstation of Beamline P10 at PETRA III/DESY. AIPConf.Proc., 1365(1):96-99, 2011.

[153] S. Kalbfleisch, M. Osterhoff, K. Giewekemeyer, H. Neubauer, S. P. Kruger, B. Hartmann, M. Bartels, M. Sprung, O. Leupold, F. Siewert, and T. Salditt. The holography endstation of beamline P10 at PETRA III. SRI 2009, 10TH INTERNATIONAL CONFERENCE ON RADIATION INSTRUMENTATION, 1234(1):433-436, 2010.

[154] Sebastian Kalbfleisch. A Dedicated Endstation for Waveguide-based X-Ray Imaging. $\mathrm{PhD}$ thesis, Universität Göttingen, 2012. 
[155] S. Kasas, G. Dumas, G. Dietler, S. Catsicas, and M. Adrian. Vitrification of cryoelectron microscopy specimens revealed by high-speed photographic imaging. J. Microsc., 211(1):48-53, 2003.

[156] Desmond Kay, editor. Techniques for electron microscopy. Blackwell, 1967.

[157] Jozef Keckes, Ingo Burgert, Klaus Fruhmann, Martin Muller, Klaas Kolln, Myles Hamilton, Manfred Burghammer, Stephan V. Roth, Stefanie StanzlTschegg, and Peter Fratzl. Cell-wall recovery after irreversible deformation of wood. Nat Mater, 2(12):810-813, December 2003.

[158] Max J. Kennedy, Sarah L. Reader, and Lisa M. Swierczynski. Preservation records of micro-organisms: evidence of the tenacity of life. Microbiology, 140(10):2513-2529, 1994.

[159] Cameron M. Kewish, Manuel Guizar-Sicairos, Chian Liu, Jun Qian, Bing Shi, Christa Benson, Ali M. Khounsary, Joan Vila-Comamala, Oliver Bunk, James R. Fienup, Albert T. Macrander, and Lahsen Assoufid. Reconstruction of an astigmatic hard X-ray beam and alignment of K-B mirrors from ptychographic coherent diffraction data. Opt. Express, 18(22):23420-23427, October 2010.

[160] Cameron M. Kewish, Pierre Thibault, Martin Dierolf, Oliver Bunk, Andreas Menzel, Joan Vila-Comamala, Konstantins Jefimovs, and Franz Pfeiffer. Ptychographic characterization of the wavefield in the focus of reflective hard X-ray optics. Ultramicroscopy, 110(4):325-329, March 2010.

[161] Janos Kirz, Chris Jacobsen, and Malcolm Howells. Soft X-ray microscopes and their biological applications. Quarterly Reviews of Biophysics, 28(01):33-130, 1995.

[162] T. Kjeldsen. Yeast secretory expression of insulin precursors. 54(3):277-286, 2000 .

[163] P. Kraft, A. Bergamaschi, Ch. Broennimann, R. Dinapoli, E. F. Eikenberry, B. Henrich, I. Johnson, A. Mozzanica, C. M. Schleputz, P. R. Willmott, and B. Schmitt. Performance of single-photon-counting PILATUS detector modules. J. Synchrotron Rad., 16(3):368-375, 2009.

[164] Anita Krisko and Miroslav Radman. Biology of extreme radiation resistance: The way of deinococcus radiodurans. Cold Spring Harbor Perspectives in Biology, 5(7):1-11, 2013.

[165] S P. Krüger, H. Neubauer, M. Bartels, S. Kalbfleisch, K. Giewekemeyer, P. J. Wilbrandt, M. Sprung, and T. Salditt. Sub-10 nm beam confinement by X-ray waveguides: design, fabrication and characterization of optical properties. J. Synchrotron Rad., 19(2):227-236, 2012. 
[166] Sven Philip Krüger. Optimization of waveguide optics for lensless x-ray imaging. PhD thesis, Universität Göttingen, 2011.

[167] Carolyn A Larabell and Keith A Nugent. Imaging cellular architecture with X-rays. Curr. Opin. Struct. Biol., 20(5):623 - 631, 2010.

[168] Werner Lauterborn and Thomas Kurz. Coherent optics : fundamentals and applications ; with 73 problems and complete solutions. Springer, 2003.

[169] Smadar Levin-Zaidman, Joseph Englander, Eyal Shimoni, Ajay K. Sharma, Kenneth W. Minton, and Abraham Minsky. Ringlike Structure of the Deinococcus radiodurans Genome: A Key to Radioresistance? Science, 299(5604):254-256, 2003.

[170] Jade Li, Joe Carroll, and David J. Ellar. Crystal structure of insecticidal $\delta$-endotoxin from bacillus thuringiensis at 2.5 à resolution. Nature, 353(6347):815-821, October 1991.

[171] H. C. Lichtenegger, M. Müller, R. Wimmer, and P. Fratzl. Microfibril Angles Inside and Outside Crossfields of Norway Spruce Tracheids. Holzforschung, 57(1):13-20, January 2003.

[172] E. Lima, A. Diaz, M. Guizar-Sicairos, S. Gorelick, P. Pernot, T. Schleier, and A. Menzel. Cryo-scanning x-ray diffraction microscopy of frozen-hydrated yeast. Journal of Microscopy, 249(1):1-7, January 2013.

[173] J J Lister. Astrosclera willeyana, the type of a new family of sponges. Zoological Results, 4:461-482, 1900.

[174] Haigang Liu, Zijian Xu, Xiangzhi Zhang, Yanqing Wu, Zhi Guo, and Renzhong Tai. Effects of missing low-frequency information on ptychographic and plane-wave coherent diffraction imaging. Appl. Opt., 52(11):2416-2427, April 2013.

[175] S. A. Livesey, A. A. del Campo, A. W. McDowall, and J. T. Stasny. Cryofixation and ultra-low-temperature freeze-drying as a preparative technique for tem. J. Microsc., 161(2):205-215, February 1991.

[176] X. Llopart, M. Campbell, R. Dinapoli, D. San Segundo, and E. Pernigotti. Medipix2: A 64k pixel readout chip with $55 \mu \mathrm{m}$ square elements working in single photon counting mode. Nuclear Science, IEEE Transactions, 49(5):2279-2283, 2002.

[177] D Russell Luke. Relaxed averaged alternating reflections for diffraction imaging. Inverse Problems, 21(1):37, 2005.

[178] Vladan Lučić, Friedrich Förster, and Wolfgang Baumeister. Structural studies by electron tomography: From cells to molecules. Annu. Rev. Biochem., 74(1):833-865, June 2005. 
[179] Thomas Mårtensson, Patrick Carlberg, Magnus Borgström, Lars Montelius, Werner Seifert, and Lars Samuelson. Nanowire arrays defined by nanoimprint lithography. Nano Lett., 4(4):699-702, March 2004.

[180] Michael T. Madigan, John M. Martinko, David A. Stahl, David P. Clark, Thomas D. Brock, Michael Thomm, and Reinhard Wirth, editors. Brock Mikrobiologie. Pearson, 2013.

[181] A. M. Maiden, M. J. Humphry, and J. M. Rodenburg. Ptychographic transmission microscopy in three dimensions using a multi-slice approach. J. Opt. Soc. Am. A, 29(8):1606-1614, August 2012.

[182] A.M. Maiden, M.J. Humphry, M.C. Sarahan, B. Kraus, and J.M. Rodenburg. An annealing algorithm to correct positioning errors in ptychography. Ultramicroscopy, 120(0):64-72, September 2012.

[183] Andrew M. Maiden and John M. Rodenburg. An improved ptychographical phase retrieval algorithm for diffractive imaging. Ultramicroscopy, 109(10):1256-1262, September 2009.

[184] Kira S. Makarova, L. Aravind, Yuri I. Wolf, Roman L. Tatusov, Kenneth W. Minton, Eugene V. Koonin, and Michael J. Daly. Genome of the extremely radiation-resistant bacterium deinococcus radiodurans viewed from the perspective of comparative genomics. Microbiol. Mol. Biol. Rev., 65(1):44-79, 2001.

[185] J.S. Maki. Bacterial intracellular sulfur globules: Structure and function. J Mol Microbiol Biotechnol, 23(4-5):270-280, 2013.

[186] Leonard Mandel and Emil Wolf. Optical coherence and quantum optics. Cambridge Univ. Press, 1995.

[187] S. Marchesini. Invited Article: A unified evaluation of iterative projection algorithms for phase retrieval. Rev. Sci. Instrum., 78(1):011301, 2007.

[188] Mareike Mathes. Sulfur Speciation in Urban Soils Studied by X-Ray Spectroscopy and Microscopy. PhD thesis, Universität Göttingen, 2014.

[189] S. Matsuyama, H. Mimura, H. Yumoto, Y. Sano, K. Yamamura, M. Yabashi, Y. Nishino, K. Tamasaku, T. Ishikawa, and K. Yamauchi. Development of scanning x-ray fluorescence microscope with spatial resolution of $30 \mathrm{~nm}$ using kirkpatrick-baez mirror optics. Rev. Sci. Instrum., 77(10):103102, October 2006.

[190] J. Clerk Maxwell. A Dynamical Theory of the Electromagnetic Field. Phil. Trans. R. Soc. Lond., 155:459-512, 1865.

[191] A.W. McDowall, W. Hofmann, J. Lepault, M. Adrian, and J. Dubochet. Cryo-electron microscopy of vitrified insect flight muscle. J. Mol. Biol., 178(1):105-111, September 1984. 
[192] J. Richard McIntosh, editor. Cellular electron microscopy. Elsevier Acad. Press, 2007.

[193] Peter T. McKenney, Adam Driks, and Patrick Eichenberger. The bacillus subtilis endospore: assembly and functions of the multilayered coat. Nat Rev Micro, 11(1):33-44, January 2013.

[194] M Meselson, J Guillemin, M Hugh-Jones, A Langmuir, I Popova, A Shelokov, and O Yampolskaya. The sverdlovsk anthrax outbreak of 1979. Science, 266(5188):1202-1208, November 1994.

[195] J. Miao, D. Sayre, and H. N. Chapman. Phase retrieval from the magnitude of the Fourier transforms of nonperiodic objects. J. Opt. Soc. Am. A, 15(6):1662-1669, 1998.

[196] J. W. Miao, R. L. Sandberg, and C. Y. Song. Coherent X-Ray Diffraction Imaging. Ieee Journal of Selected Topics In Quantum Electronics, 18(1):399410, January 2012.

[197] Jianwei Miao, Pambos Charalambous, Janos Kirz, and David Sayre. Extending the methodology of X-ray crystallography to allow imaging of micrometre-sized non-crystalline specimens. Nature, 400(6742):342-344, July 1999.

[198] R. P. Millane. Phase retrieval in crystallography and optics. J. Opt. Soc. Am. A, 7(3):394-411, 1990.

[199] H. Mimura, T. Kimura, H. Yokoyama, H. Yumoto, S. Matsuyama, K. Tamasaku, Y. Koumura, M. Yabashi, T. Ishikawa, and K. Yamauchi. Development of an Adaptive Optical System for Sub-10-nm Focusing of Synchrotron Radiation Hard X-rays. AIP Conf. Proc., 1365:13-17, 2011.

[200] Hidekazu Mimura, Soichiro Handa, Takashi Kimura, Hirokatsu Yumoto, Daisuke Yamakawa, Hikaru Yokoyama, Satoshi Matsuyama, Kouji Inagaki, Kazuya Yamamura, Yasuhisa Sano, Kenji Tamasaku, Yoshinori Nishino, Makina Yabashi, Tetsuya Ishikawa, and Kazuto Yamauchi. Breaking the $10 \mathrm{~nm}$ barrier in hard-X-ray focusing. Nat Phys, 6(2):122-125, February 2010 .

[201] Hidekazu Mimura, Hirokatsu Yumoto, Satoshi Matsuyama, Yasuhisa Sano, Kazuya Yamamura, Yuzo Mori, Makina Yabashi, Yoshinori Nishino, Kenji Tamasaku, Tetsuya Ishikawa, and Kazuto Yamauchi. Efficient focusing of hard x rays to $25 \mathrm{~nm}$ by a total reflection mirror. $A P L, 90(5): 051903,2007$.

[202] Abraham Minsky, Eyal Shimoni, and Joseph Englander. Rebuttal: Study of the Deinococcus radiodurans Nucleoid. J. Bacteriol., 188(17):6059, 2006.

[203] Abraham Minsky, Eyal Shimoni, and Joseph Englander. Ring-like nucleoids and dna repair through error-free nonhomologous end joining in deinococcus radiodurans. Journal of Bacteriology, 188(17):6047-6051, 2006. 
[204] J. Miot, K. Benzerara, G. Morin, S. Bernard, O. Beyssac, E. Larquet, A. Kappler, and F. Guyot. Transformation of vivianite by anaerobic nitratereducing iron-oxidizing bacteria. Geobiology, 7(3):373-384, 2009.

[205] A. Moir. How do spores germinate? J. Appl. Microbiol., 101(3):526-530, 2006.

[206] Sheilagh Molloy. Symbiotic exceptions. Nat Rev Micro, 5(7):473-473, July 2007.

[207] G. Morrison, W.J. Eaton, R. Barrett, and P. Charalambous. STXM imaging with a configured detector. J. Phys. IV France, 104:547-550, March 2003.

[208] G.R. Morrison and B. Niemann. Differential phase contrast x-ray microscopy. In Jürgen Thieme, editor, X-ray microscopy and spectromicroscopy, Würzburg, 1996, pages I - 85-94, Berlin, 1998. Springer.

[209] M. Müller, M. Burghammer, D. Flot, C. Riekel, C. Morawe, B. Murphy, and A. Cedola. Microcrystallography with an x-ray waveguide. J. Appl. Crystallogr., 33(5):1231-1240, 2000.

[210] P. D. Nellist, B. C. McCallum, and J. M. Rodenburg. Resolution beyond the 'information limit' in transmission electronmicroscopy. Nature, 374(6523):630-632, April 1995.

[211] Johanna Nelson, Xiaojing Huang, Jan Steinbrener, David Shapiro, Janos Kirz, Stefano Marchesini, Aaron M. Neiman, Joshua J. Turner, and Chris Jacobsen. High-resolution x-ray diffraction microscopy of specifically labeled yeast cells. PNAS, 107(16):7235-7239, April 2010.

[212] H. Neubauer, S. Hoffmann, M. Kanbach, J. Haber, S. Kalbfleisch, S. P. Krüger, and T. Salditt. High aspect ratio x-ray waveguide channels fabricated by e-beam lithography and wafer bonding. J. Appl. Phys., 115(21):214305, 2014.

[213] W. L. Nicholson. Roles of bacillus endospores in the environment. Cell. Mol. Life. Sci., 59(3):410-416, 2002.

[214] Wayne L. Nicholson, Nobuo Munakata, Gerda Horneck, Henry J. Melosh, and Peter Setlow. Resistance of bacillus endospores to extreme terrestrial and extraterrestrial environments. Microbiol. Mol. Biol. Rev., 64(3):548-572, 2000 .

[215] PH Nielsen, MA De Muro, and JL Nielsen. Studies on the in situ physiology of thiothrix spp. present in activated sludge. Environ. Microbiol., 2:389-398, 2000 .

[216] Keith A. Nugent. Coherent methods in the X-ray sciences. Adv. Phys., $59(1): 1-99,2010$. 
[217] K. Nygård, O. Bunk, E. Perret, C. David, and J. F. van der Veen. Diffraction gratings as small-angle X-ray scattering calibration standards. Journal of Applied Crystallography, 43(2):350-351, 2010.

[218] M. Obst, J. Wang, and A. P. Hitchcock. Soft x-ray spectro-tomography study of cyanobacterial biomineral nucleation. Geobiology, 7(5):577-591, 2009.

[219] Martin Obst and Gregor Schmid. 3d chemical mapping: Application of scanning transmission (soft) x-ray microscopy (stxm) in combination with angle-scan tomography in bio-, geo-, and environmental sciences. In John Kuo, editor, Methods in Molecular Biology, volume 1117, pages 757-781. Humana Press, 2014.

[220] Anna Ohradanova, Daniela Vullo, Silvia Pastorekova, Jaromir Pastorek, Daniel J. Jackson, Gert Wörheide, and Claudiu T. Supuran. Anion inhibition studies of an $\alpha$-carbonic anhydrase from the living fossil astrosclera willeyana. Bioorganic \& Medicinal Chemistry Letters, 22(3):1314-1316, February 2012.

[221] C. Ollinger, C. Fuhse, S. Kalbfleisch, R. Tucoulou, and T. Salditt. Object localization with $10 \mathrm{~nm}$ accuracy by x-ray phase contrast projection imaging. Applied Physics Letters, 91(5):051110, 2007.

[222] Markus Osterhoff and Tim Salditt. Coherence filtering of x-ray waveguides: analytical and numerical approach. New Journal of Physics, 13(10):103026, 2011.

[223] D. M. Paganin. Coherent X-Ray Optics. New York: Oxford University Press, 2006.

[224] O. Paris, D. Loidl, H. Peterlik, M. Muller, H. Lichtenegger, and P. Fratzl. The internal structure of single carbon fibers determined by simultaneous small- and wide-angle scattering. Journal of Applied Crystallography, 33(3 Part 1):695-699, 2000.

[225] D Pennicard and H Graafsma. Simulated performance of high-z detectors with medipix3 readout. Journal of Instrumentation, 6(06):P06007, 2011.

[226] D Pennicard, S Lange, S Smoljanin, H Hirsemann, and H Graafsma. Lambda - large area medipix3-based detector array. Journal of Instrumentation, 7(11):C11009, 2012.

[227] David Pennicard, Sabine Lange, Sergej Smoljanin, Julian Becker, Helmut Hirsemann, Michael Epple, and Heinz Graafsma. Development of lambda: Large area medipix-based detector array. Journal of Instrumentation, 6(11):C11009, 2011.

[228] F. Pfeiffer, C. David, M. Burghammer, C. Riekel, and T. Salditt. TwoDimensional X-ray Waveguides and Point Sources. Science, 297(6):230, 2002. 
[229] Patrick J Piggot and David W Hilbert. Sporulation of bacillus subtilis. Curr. Opin. Microbiol., 7(6):579-586, December 2004.

[230] Marco Plomp, Terrance J. Leighton, Katherine E. Wheeler, Haley D. Hill, and Alexander J. Malkin. In vitro high-resolution structural dynamics of single germinating bacterial spores. PNAS, 104(23):9644-9649, 2007.

[231] Marco Plomp, Terrance J. Leighton, Katherine E. Wheeler, and Alexander J. Malkin. Architecture and high-resolution structure of bacillus thuringiensis and bacillus cereus spore coat surfaces. Langmuir, 21(17):7892-7898, July 2005.

[232] Marco Plomp, Terrance J. Leighton, Katherine E. Wheeler, and Alexander J. Malkin. The high-resolution architecture and structural dynamics of bacillus spores. $B J, 88(1): 603-608$, January 2005.

[233] Kit Pogliano, Elizabeth Harry, and Richard Losick. Visualization of the subcellular location of sporulation proteins in bacillus subtilis using immunofluorescence microscopy. Mol. Microbiol., 18(3):459-470, November 1995.

[234] C Ponchut, J M Rigal, J Clément, E Papillon, A Homs, and S Petitdemange. MAXIPIX, a fast readout photon-counting X-ray area detector for synchrotron applications. Journal of Instrumentation, 6(01):C01069, 2011.

[235] Cyril Ponchut, Joël Clément, Jean-Marie Rigal, Emmanuel Papillon, John Vallerga, Daniel LaMarra, and Bettina Mikulec. Photon-counting X-ray imaging at kilohertz frame rates. Nucl. Instrum. Methods Phys. Res., 576(1):109-112, June 2007.

[236] G. Potdevin, U. Trunk, and H. Graafsma. Performance simulation of a detector for 4th generation photon sources: The agipd. Nucl. Instrum. Methods Phys. Res., 607(1):51-54, August 2009.

[237] Stephan Preibisch, Stephan Saalfeld, and Pavel Tomancak. Globally optimal stitching of tiled 3D microscopic image acquisitions. Bioinformatics, 25(11):1463-1465, 2009. fiji stitching.

[238] William H. Press, Saul A. Teukolsky, William T. Vetterling, and Brian P. Flannery. Numerical recipes : the art of scientific computing. Cambridge Univ. Press, third edition, 2007.

[239] Marius Priebe. PhD thesis, Universität Göttingen, In preparation.

[240] Marius Priebe, Marten Bernhardt, Christoph Blum, Marco Tarantola, Eberhard Bodenschatz, and Tim Salditt. Scanning X-Ray Nanodiffraction on Dictyostelium discoideum. Biophys J, 107(11):2662-2673, December 2014. 
[241] Corey T. Putkunz, Jesse N. Clark, David J. Vine, Garth J. Williams, Mark A. Pfeifer, Eugeniu Balaur, Ian McNulty, Keith A. Nugent, and Andrew G. Peele. Phase-Diverse Coherent Diffractive Imaging: High Sensitivity with Low Dose. Phys. Rev. Lett., 106(1):013903, January 2011.

[242] N. Quéric and J. Reitner et al. Geomicrobiology Journal, unpublished manuscript, 2014.

[243] H. M. Quiney. Coherent diffractive imaging using short wavelength light sources. Journal of Modern Optics, 57(13):1109-1149, 2010.

[244] C. Quintana. Cryofixation, cryosubstitution, cryoembedding for ultrastructural, immunocytochemical and microanalytical studies. Micron, 25(1):6399, 1994.

[245] J. Radon. Über die Bestimmung von Funktionen durch ihre Integralwerte längs gewisser Mannigfaltigkeiten. Akad. Wiss., 69:262-277, 1917.

[246] W G Rees. The validity of the Fresnel approximation. European Journal of Physics, 8(1):44-48, 1987.

[247] J. Reitner. 'coralline spongien' - der versuch einer phylogenetischtaxonomischen analyse. Berliner geowissenschaftliche Abhandlungen Reihe E (Paläobiologie), 1:1-352, 1992.

[248] S. Rinnerthaler, P. Roschger, H. F. Jakob, A. Nader, K. Klaushofer, and P. Fratzl. Scanning Small Angle X-ray Scattering Analysis of Human Bone Sections. Calcified Tissue International, 64(5):422-429, 1999.

[249] J. M. Rodenburg and H. M. L. Faulkner. A phase retrieval algorithm for shifting illumination. Appl. Phys. Lett., 85(20):4795-4797, November 2004.

[250] J. M. Rodenburg, A. C. Hurst, A. G. Cullis, B. R. Dobson, F. Pfeiffer, O. Bunk, C. David, K. Jefimovs, and I. Johnson. Hard-X-Ray Lensless Imaging of Extended Objects. Phys. Rev. Lett., 98(3):034801, January 2007.

[251] J.M. Rodenburg. Ptychography and Related Diffractive Imaging Methods. Advances in Imaging and Electron Physics, Volume 150:87-184, 2008.

[252] Albert Rose. A unified approach to the performance of photographic film, television pickup tubes, and the human eye. J. Soc. Mot. Picture Eng., 47(4):273-294, October 1946.

[253] Heather Rothfuss, Jimmie C. Lara, Amy K. Schmid, and Mary E. Lidstrom. Involvement of the S-layer proteins Hpi and Slpa in the maintenance of cell envelope integrity in deinococcus radiodurans $R_{1}$. Microbiology, 152(9):2779$2787,2006$.

[254] Edward M. Rubin. Genomics of cellulosic biofuels. Nature, 454(7206):841845, August 2008. 
[255] T.P. Russell. X-ray and neutron reflectivity for the investigation of polymers. Materials Science Reports, 5(4):171-271, 1990.

[256] Anne Sakdinawat and David Attwood. Nanoscale X-ray imaging. Nat Photon, 4(12):840-848, December 2010.

[257] T. Salditt, K. Giewekemeyer, C. Fuhse, S. P. Kruger, R. Tucoulou, and P. Cloetens. Projection phase contrast microscopy with a hard x-ray nanofocused beam: Defocus and contrast transfer. Phys. Rev. B, 79(18):184112-6, May 2009.

[258] T. Salditt, S. Kalbfleisch, M. Osterhoff, S. P. Krüger, M. Bartels, K. Giewekemeyer, H. Neubauer, and M. Sprung. Partially coherent nano-focused x-ray radiation characterized by Talbot interferometry. Opt. Express, 19(10):96569675, May 2011.

[259] Bahaa E. A. Saleh and Malvin Carl Teich. Fundamentals of Photonics. Wiley, 1991.

[260] A Schavkan, F Westermeier, A Zozulya, S Bondarenko, G Grübel, C Schroer, and M Sprung. Using the maxipix detector for coherent x-ray scattering applications. Journal of Physics: Conference Series, 425(20):202004, 2013.

[261] Johannes Schindelin, Ignacio Arganda-Carreras, Erwin Frise, Verena Kaynig, Mark Longair, Tobias Pietzsch, Stephan Preibisch, Curtis Rueden, Stephan Saalfeld, Benjamin Schmid, Jean-Yves Tinevez, Daniel James White, Volker Hartenstein, Kevin Eliceiri, Pavel Tomancak, and Albert Cardona. Fiji: an open-source platform for biological-image analysis. Nat Meth, 9(7):676-682, July 2012.

[262] Caroline A Schneider, Wayne S Rasband, and Kevin W Eliceiri. Nih image to imagej: 25 years of image analysis. Nat Meth, 9(7):671-675, July 2012.

[263] E. Schnepf, N. Crickmore, J. Van Rie, D. Lereclus, J. Baum, J. Feitelson, D. R. Zeigler, and D. H. Dean. Bacillus thuringiensis and its pesticidal crystal proteins. Microbiol. Mol. Biol. Rev., 62(3):775-806, 1998.

[264] C. G. Schroer, O. Kurapova, J. Patommel, P. Boye, J. Feldkamp, B. Lengeler, M. Burghammer, C. Riekel, L. Vincze, A. van der Hart, and M. Kuchler. Hard x-ray nanoprobe based on refractive x-ray lenses. Appl. Phys. Lett., 87(12):124103-3, September 2005.

[265] A. Schropp, P. Boye, J. M. Feldkamp, R. Hoppe, J. Patommel, D. Samberg, S. Stephan, K. Giewekemeyer, R. N. Wilke, T. Salditt, J. Gulden, A. P. Mancuso, I. A. Vartanyants, E. Weckert, S. Schoder, M. Burghammer, and C. G. Schroer. Hard x-ray nanobeam characterization by coherent diffraction microscopy. Appl. Phys. Lett., 96(9):091102, March 2010. 
[266] A. Schropp, R. Hoppe, J. Patommel, D. Samberg, F. Seiboth, S. Stephan, G. Wellenreuther, G. Falkenberg, and C. G. Schroer. Hard x-ray scanning microscopy with coherent radiation: Beyond the resolution of conventional x-ray microscopes. Appl. Phys. Lett., 100(25):253112, June 2012.

[267] Andreas Schropp. Experimental Coherent X-Ray Diffractive Imaging : Capabilities and Limitations of the Technique. PhD thesis, Universität Hamburg, 2008.

[268] Andreas Schropp, Robert Hoppe, Vivienne Meier, Jens Patommel, Frank Seiboth, Hae Ja Lee, Bob Nagler, Eric C. Galtier, Brice Arnold, Ulf Zastrau, Jerome B. Hastings, Daniel Nilsson, Fredrik Uhlen, Ulrich Vogt, Hans M. Hertz, and Christian G. Schroer. Full spatial characterization of a nanofocused x-ray free-electron laser beam by ptychographic imaging. Sci. Rep., 3:1633, April 2013.

[269] Andreas Schropp and Christian G Schroer. Dose requirements for resolving a given feature in an object by coherent x-ray diffraction imaging. New Journal of Physics, 12(3):035016, 2010.

[270] M. C. Scott, Chien-Chun Chen, Matthew Mecklenburg, Chun Zhu, Rui Xu, Peter Ercius, Ulrich Dahmen, B. C. Regan, and Jianwei Miao. Electron tomography at 2.4-angstrom resolution. Nature, 483(7390):444-447, March 2012.

[271] Julia Sedlmair. Soft x-ray spectromicroscopy of environmental and biological samples. PhD thesis, Universität Göttingen, 2011.

[272] Peter Setlow. Spore germination. Curr. Opin. Microbiol., 6(6):550-556Z, December 2003.

[273] C.E. Shannon. Communication in the Presence of Noise. Proceedings of the IRE, 37(1):10-21, 1949.

[274] David Shapiro, Pierre Thibault, Tobias Beetz, Veit Elser, Malcolm Howells, Chris Jacobsen, Janos Kirz, Enju Lima, Huijie Miao, Aaron M. Neiman, and David Sayre. Biological imaging by soft x-ray diffraction microscopy. PNAS, 102(43):15343-15346, 2005.

[275] George C. Sherman. Application of the Convolution Theorem to Rayleigh's Integral Formulas. J. Opt. Soc. Am., 57(4):546-547, April 1967.

[276] V. B. D. Skerman, Galina Dementjeva, and Barbara J. Carey. Intracellular deposition of sulfur by sphaerotilus natans. J. Bacteriol., 73(4):504-512, 1957.

[277] Dea Slade and Miroslav Radman. Oxidative Stress Resistance in Deinococcus radiodurans. Microbiology and Molecular Biology Reviews, 75(1):133191, 2011. 
[278] U B Sleytr and P Messner. Crystalline surface layers on bacteria. Annu. Rev. Microbiol., 37(1):311-339, October 1983.

[279] Uwe B Sleytr and Terry J Beveridge. Bacterial s-layers. Trends Microbiol., $7(6): 253-260$, June 1999.

[280] V.A. Solé, E. Papillon, M. Cotte, Ph. Walter, and J. Susini. A multiplatform code for the analysis of energy-dispersive X-ray fluorescence spectra. Spectrochimica Acta Part B: Atomic Spectroscopy, 62(1):63-68, January 2007.

[281] R C Spencer. Bacillus anthracis. Journal of Clinical Pathology, 56(3):182187, 2003.

[282] Erko Stackebrandt and Carl R. Woese. Towards a phylogeny of the actinomycetes and related organisms. Curr. Microbiol., 5(4):197-202, 1981.

[283] Erik P. Sunde, Peter Setlow, Lars Hederstedt, and Bertil Halle. The physical state of water in bacterial spores. PNAS, 106(46):19334-19339, 2009.

[284] Akihiro Suzuki, Shin Furutaku, Kei Shimomura, Kazuto Yamauchi, Yoshiki Kohmura, Tetsuya Ishikawa, and Yukio Takahashi. High-resolution multislice x-ray ptychography of extended thick objects. Phys. Rev. Lett., 112(5):053903, February 2014.

[285] Y. Takahashi. Multiscale element mapping of buried structures by ptychographic x-ray diffraction microscopy using anomalous scattering. Applied Physics Letters, 99(13):131905, 2011.

[286] Yukio Takahashi, Akihiro Suzuki, Shin Furutaku, Kazuto Yamauchi, Yoshiki Kohmura, and Tetsuya Ishikawa. High-resolution and high-sensitivity phasecontrast imaging by focused hard x-ray ptychography with a spatial filter. Appl. Phys. Lett., 102(9):094102-4, March 2013.

[287] Yukio Takahashi, Akihiro Suzuki, Nobuyuki Zettsu, Yoshiki Kohmura, Yasunori Senba, Haruhiko Ohashi, Kazuto Yamauchi, and Tetsuya Ishikawa. Towards high-resolution ptychographic x-ray diffraction microscopy. Phys. Rev. B, 83(21):214109, June 2011.

[288] Hiroaki Takajo, Tohru Takahashi, Hiroaki Kawanami, and Ryuzo Ueda. Numerical investigation of the iterative phase-retrieval stagnation problem: territories of convergence objects and holes in their boundaries. J. Opt. Soc. Am. A, 14(12):3175-3187, 1997.

[289] M W Tate, D Chamberlain, K S Green, H T Philipp, P Purohit, C Strohman, and S M Gruner. A medium-format, mixed-mode pixel array detector for kilohertz x-ray imaging. Journal of Physics: Conference Series, 425(6):062004, 2013. 
[290] Alexis Templeton and Emily Knowles. Microbial transformations of minerals and metals: Recent advances in geomicrobiology derived from synchrotronbased x-ray spectroscopy and x-ray microscopy. Annu. Rev. Earth Planet. Sci., 37(1):367-391, April 2009.

[291] Rudolf K. Thauer, Anne-Kristin Kaster, Henning Seedorf, Wolfgang Buckel, and Reiner Hedderich. Methanogenic archaea: ecologically relevant differences in energy conservation. Nat Rev Micro, 6(8):579-591, August 2008.

[292] Philippe Thévenaz and Michael Unser. User-friendly semiautomated assembly of accurate image mosaics in microscopy. Microsc. Res. Tech., 70(2):135146, February 2007.

[293] Pierre Thibault. Algorithmic methods in diffraction microscopy. PhD thesis, University Microfilms International, Ann Arbor, Mich., 2008.

[294] Pierre Thibault, Martin Dierolf, Oliver Bunk, Andreas Menzel, and Franz Pfeiffer. Probe retrieval in ptychographic coherent diffractive imaging. Ultramicroscopy, 109(4):338-343, March 2009.

[295] Pierre Thibault, Martin Dierolf, Cameron M. Kewish, Andreas Menzel, Oliver Bunk, and Franz Pfeiffer. Contrast mechanisms in scanning transmission x-ray microscopy. Phys. Rev. A, 80(4):043813, 2009.

[296] Pierre Thibault, Martin Dierolf, Andreas Menzel, Oliver Bunk, Christian David, and Franz Pfeiffer. High-Resolution Scanning X-ray Diffraction Microscopy. Science, 321(5887):379-382, 2008.

[297] Pierre Thibault and Veit Elser. X-Ray Diffraction Microscopy. Annu. Rev. Condens. Matter Phys., 1(1):237-255, July 2010.

[298] Pierre Thibault, Manuel Guizar-Sicairos, and Andreas Menzel. Coherent imaging at the diffraction limit. J. Synchrotron Rad., 21(5):1011-1018, 2014.

[299] Pierre Thibault and Andreas Menzel. Reconstructing state mixtures from diffraction measurements. Nature, 494(7435):68-71, February 2013.

[300] Jürgen Thieme, editor. X-ray microscopy and spectromicroscopy : status report from the Fifth International Conference, Würzburg, August 19 - 23, 1996 ; [based on presentations to the International Conference of X-Ray Microscopy and Spectromicroscopy XRM 96]. Springer, 1998.

[301] Jürgen Thieme, Gerd Schneider, and Christian Knöchel. X-ray tomography of a microhabitat of bacteria and other soil colloids with sub-100 nm resolution. Micron, 34(6-7):339-344, 2003.

[302] Albert C. Thompson, Janos Kirz, David T. Attwood, Eric M. Gullikson, Malcolm R. Howells, Yanwei Liu Kortright, Arthur L. Robinson, James H. Underwood, Kwang-Je Kim, Ingolf Lindau, Piero Pianetta, Herman Winick, 
Gwyn P. Williams, and James H. Scofield. X-ray Data Booklet. Center for X-ray Optics and Advanced Light Source, Lawrence Berkeley National Laboratory University of California Berkeley, CA 94720, third edition, 2009.

[303] B. J. Thompson and E. Wolf. Two-beam interference with partially coherent light. J. Opt. Soc. Am., 47(10):895-902, 1957.

[304] L S Tisa, T Koshikawa, and P Gerhardt. Wet and dry bacterial spore densities determined by buoyant sedimentation. Appl. Environ. Microbiol., 43(6):1307-1310, 1982.

[305] William F. Tivol, Ariane Briegel, and Grant J. Jensen. An Improved Cryogen for Plunge Freezing. Microscopy and Microanalysis, 14(05):375-379, 2008.

[306] H. Toyokawa, Ch. Broennimann, E.F. Eikenberry, B. Henrich, M. Kawase, M. Kobas, P. Kraft, M. Sato, B. Schmitt, M. Suzuki, H. Tanida, and T. Uruga. Single photon counting pixel detectors for synchrotron radiation experiments. Nucl. Instrum. Methods Phys. Res., Sect. A, 623(1):204-206, November 2010.

[307] Ashish Tripathi, Ian McNulty, and Oleg G Shpyrko. Ptychographic overlap constraint errors and the limits of their numerical recovery using conjugate gradient descent methods. Opt. Express, 22(2):1452-1466, 2014.

[308] P. Trueb, B. A. Sobott, R. Schnyder, T. Loeliger, M. Schneebeli, M. Kobas, R. P. Rassool, D. J. Peake, and C. Broennimann. Improved count rate corrections for highest data quality with pilatus detectors. J. Synchrotron Rad., 19(3):347-351, 2012.

[309] Edit Urban, Sonja Jacob, Maria Nemethova, Guenter P. Resch, and J. Victor Small. Electron tomography reveals unbranched networks of actin filaments in lamellipodia. Nat Cell Biol, 12(5):429-435, May 2010.

[310] Malte Julian Udo Vaßholz. Nano-focus x-ray diffraction: Increasing the dynamic range with semi-transparent beamstops. Master's thesis, GeorgAugust Universität Göttingen, 2012.

[311] W. Vernon, M. Allin, R. Hamlin, T. Hontz, D. Nguyen, F. Augustine, S. M. Gruner, Ng. H. Xuong, D. R. Schuette, M. W. Tate, and L. J. Koerner. First results from the $128 \times 128$ pixel mixed-mode si x-ray detector chip. Proc. SPIE, 6706:67060U-67060U-11, 2007.

[312] Joan Vila-Comamala, Ana Diaz, Manuel Guizar-Sicairos, Alexandre Mantion, Cameron M. Kewish, Andreas Menzel, Oliver Bunk, and Christian David. Characterization of high-resolution diffractive X-ray optics by ptychographic coherent diffractive imaging. Opt. Express, 19(22):21333-21344, October 2011. 
[313] Joan Vila-Comamala, Sergey Gorelick, Elina Färm, Cameron M. Kewish, Ana Diaz, Ray Barrett, Vitaliy A. Guzenko, Mikko Ritala, and Christian David. Ultra-high resolution zone-doubled diffractive X-ray optics for the multi-keV regime. Opt. Express, 19(1):175-184, Jan 2011.

[314] Joan Vila-Comamala, Yongsheng Pan, Jeffrey J. Lombardo, William M. Harris, Wilson K. S. Chiu, Christian David, and Yuxin Wang. Zone-doubled fresnel zone plates for high-resolution hard x-ray full-field transmission microscopy. J. Synchrotron Rad., 19(5):705-709, 2012.

[315] D. J. Vine, D. Pelliccia, C. Holzner, S. B. Baines, A. Berry, I. McNulty, S. Vogt, A. G. Peele, and K. A. Nugent. Simultaneous x-ray fluorescence and ptychographic microscopy of cyclotella meneghiniana. Opt. Express, 20(16):18287-18296, 2012.

[316] D. J. Vine, G. J. Williams, B. Abbey, M. A. Pfeifer, J. N. Clark, M. D. de Jonge, I. McNulty, A. G. Peele, and K. A. Nugent. Ptychographic Fresnel coherent diffractive imaging. Phys. Rev. A, 80(6):063823, December 2009.

[317] David G. Voelz and Michael C. Roggemann. Digital simulation of scalar optical diffraction: revisiting chirp function sampling criteria and consequences. Appl. Opt., 48(32):6132-6142, 2009.

[318] W. Wagermaier, H. S. Gupta, A. Gourrier, M. Burghammer, P. Roschger, and P. Fratzl. Spiral twisting of fiber orientation inside bone lamellae. Biointerphases, 1(1):1-5, March 2006.

[319] Jesper Wallentin. Doping of Semiconductor Nanowires. PhD thesis, Lund University, 2013.

[320] Jesper Wallentin, Nicklas Anttu, Damir Asoli, Maria Huffman, Ingvar Åberg, Martin H. Magnusson, Gerald Siefer, Peter Fuss-Kailuweit, Frank Dimroth, Bernd Witzigmann, H. Q. Xu, Lars Samuelson, Knut Deppert, and Magnus T. Borgström. Inp nanowire array solar cells achieving $13.8 \%$ efficiency by exceeding the ray optics limit. Science, 339(6123):1057-1060, 2013.

[321] U. Weierstall, Q. Chen, J. C. H. Spence, M. R. Howells, M. Isaacson, and R. R. Panepucci. Image reconstruction from electron and X-ray diffraction patterns using iterative algorithms: experiment and simulation. Ultramicroscopy, 90(2-3):171-195, February 2002.

[322] Britta Weinhausen. Scanning X-ray nano-diffraction on eukaryotic cells: from freeze-dried to living cells. $\mathrm{PhD}$ thesis, Georg-August Universität Göttingen, 2013.

[323] Britta Weinhausen, Oliva Saldanha, Robin N. Wilke, Christian Dammann, Marius Priebe, Manfred Burghammer, Michael Sprung, and Sarah Köster. Scanning x-ray nanodiffraction on living eukaryotic cells in microfluidic environments. Phys. Rev. Lett., 112(8):088102, February 2014. 
[324] Timm Weitkamp. Imaging and tomography with high resolution using coherent hard synchroton radiation. PhD thesis, University of Hamburg, 2002.

[325] Andrew J. Westphal, P. Buford Price, Terrance J. Leighton, and Katherine E. Wheeler. Kinetics of size changes of individual bacillus thuringiensis spores in response to changes in relative humidity. PNAS, 100(6):3461-3466, 2003 .

[326] Owen White, Jonathan A. Eisen, John F. Heidelberg, Erin K. Hickey, Jeremy D. Peterson, Robert J. Dodson, Daniel H. Haft, Michelle L. Gwinn, William C. Nelson, Delwood L. Richardson, Kelly S. Moffat, Haiying Qin, Lingxia Jiang, Wanda Pamphile, Marie Crosby, Mian Shen, Jessica J. Vamathevan, Peter Lam, Lisa McDonald, Terry Utterback, Celeste Zalewski, Kira S. Makarova, L. Aravind, Michael J. Daly, Kenneth W. Minton, Robert D. Fleischmann, Karen A. Ketchum, Karen E. Nelson, Steven Salzberg, Hamilton O. Smith, J. Craig, Venter, and Claire M. Fraser. Genome Sequence of the Radioresistant Bacterium Deinococcus radiodurans R1. Science, 286(5444):1571-1577, November 1999.

[327] E. T. Whittaker. On the functions which are represented by the expansions of the interpolation-theory. Edinburgh University, Edinburgh, 1915.

[328] R. N. Wilke. Zur Phasenrekonstruktion von X-Feldern mit gekrümmten Phasenfronten. Diplomarbeit, Universität Göttingen. 2010.

[329] R. N. Wilke, M. Hoppert, M. Krenkel, M. Bartels, and T. Salditt. Quantitative X-Ray Phase Contrast Waveguide-Imaging of Bacterial Endospores. J. Appl. Crystallogr., submitted manuscript, 2014.

[330] R. N. Wilke, M. Priebe, M. Bartels, K. Giewekemeyer, A. Diaz, P. Karvinen, and T. Salditt. Hard X-ray imaging of bacterial cells: nano-diffraction and ptychographic reconstruction. Opt. Express, 20(17):19232-19254, August 2012.

[331] R. N. Wilke, N. Quéric, M. Hoppert, C. Heller, A. Schropp, C.G. Schroer, M. Burghammer, T. Salditt, and J. Reitner. Scanning hard x-ray microscopy imaging modalities for geobiological samples. Geomicrobiology Journal, accepted for publication, 2014.

[332] R. N. Wilke, M. Vassholz, and T. Salditt. Semi-transparent central stop in high-resolution x-ray ptychography using kirkpatrick-baez focusing. Acta Crystallogr.A, 69(5):490-497, 2013.

[333] R. N. Wilke, J. Wallentin, M. Osterhoff, D. Pennicard, A. Zozulya, M. Sprung, and T. Salditt. High flux ptychographic imaging using the new $55 \mu \mathrm{m}$-pixel detector 'lambda' based on the medipix3 readout chip. Acta Crystallogr.A, 70:552-562, 2014. 
[334] G. J. Williams, H. M. Quiney, B. B. Dhal, C. Q. Tran, K. A. Nugent, A. G. Peele, D. Paterson, and M. D. de Jonge. Fresnel Coherent Diffractive Imaging. Phys. Rev. Lett., 97(2):025506, 2006.

[335] Garth J. Williams, Harry M. Quiney, Andrew G. Peele, and Keith A. Nugent. Coherent diffractive imaging and partial coherence. Phys. Rev. B, 75(10):104102, March 2007.

[336] S. Williams, X. Zhang, C. Jacobsen, J. Kirz, S. Lindaas, J. Van't Hof, and S. S. Lamm. Measurements of wet metaphase chromosomes in the scanning transmission x-ray microscope. J. Microsc., 170(2):155-165, May 1993.

[337] Emil Wolf. Introduction to the theory of coherence and polarization of light. Cambridge Univ. Press, Cambridge [u.a.], 2007.

[338] Gert Wörheide. The reef cave dwelling ultraconservative coralline demosponge Astrosclera willeyana Lister 1900 from the Indo-Pacific. Facies, 38(1):1-88, December 1998.

[339] Takashi Yamamoto and Roy E. McLaughlin. Isolation of a protein from the parasporal crystal of bacillus thuringiensis var, kurstaki toxic to the mosquito larva, aedes taeniorhynchus. Biochem. Biophys. Res. Commun., 103(2):414-421, November 1981.

[340] S. Zabler, P. Cloetens, J.-P. Guigay, J. Baruchel, and M. Schlenker. Optimization of phase contrast imaging using hard $\mathrm{x}$ rays. Rev. Sci. Instrum., 76(7):073705, 2005.

[341] F. Zernike. The concept of degree of coherence and its application to optical problems. Physica, 5(8):785-795, August 1938.

[342] Julie Zimmerman and John Battista. A ring-like nucleoid is not necessary for radioresistance in the Deinococcaceae. BMC Microbiology, 5(1):17, 2005.

[343] A V Zozulya, A Shabalin, H Schulte-Schrepping, J Heuer, M Spiwek, I Sergeev, I Besedin, I A Vartanyants, and M Sprung. Wavefront preserving channel-cut optics for coherent x-ray scattering experiments at the p10 beamline at petraiii. J. Phys: Conf. Ser., 499(1):012003, 2014. 



\section{Original (Peer-reviewed) Publications:}

- R. N. Wilke, M. Priebe, M. Bartels, K. Giewekemeyer, A. Diaz, P. Karvinen and T. Salditt

Hard X-ray imaging of bacterial cells: nano-diffraction and ptychographic reconstruction

Optics Express, Vol. 20 Issue 17, pp. 19232-19254 (2012).

http://dx.doi.org/10.1364/0E.20.019232

- R. N. Wilke, M. Vassholz and T. Salditt

Semi-transparent central stop in high-resolution X-ray ptychography using Kirkpatrick-Baez focusing

Acta Crystallographica A69, pp. 490-497 (2013). http://dx.doi.org/10.1107/S0108767313019612

- R. N. Wilke, N. Quéric, M. Hoppert, C. Heller, A. Schropp, C.G. Schroer, M. Burghammer, T. Salditt and J. Reitner

Scanning Hard X-ray Microscopy Imaging Modalities for Geobiological Samples

Geomicrobiology Journal (2014). In the press.

ID: 908982 DOI:10.1080/01490451.2014.908982

- R. N. Wilke, J. Wallentin, M. Osterhoff, D. Pennicard, A. Zozulya, M. Sprung and T. Salditt

High Flux Ptychographic Imaging using the new $55 \mu \mathrm{m}$-pixel detector 'Lambda' based on the Medipix3 readout chip

Acta Crystallographica A70, (2014).

http://dx.doi.org/10.1107/S2053273314014545

- M. Bartels, M. Krenkel, J. Haber, R.N. Wilke, and T. Salditt

$X$-ray holographic imaging of hydrated biological cells in solution

Physical Review Letters (2014). Accepted for publication.

- M. Osterhoff, C. Eberl, F. Doring, R. N. Wilke, J. Wallentin, H.-U. Krebs, M. Sprung and T. Salditt

Towards multi-order hard x-ray imaging with multilayer zone plates Journal of Applied Crystallography (2014). Accepted for publication.

- J. Wallentin, M. Osterhoff, R. N. Wilke, K.-M. Persson, L.-E. Wernersson, M. Sprung, and T. Salditt

Hard X-ray Detection Using a Single $100 \mathrm{~nm}$ Diameter Nanowire

Nano Letters (2014). In the press.

http://dx.doi.org/10.1021/nl5040545

- K. Giewekemeyer, H. T. Philipp, R. N. Wilke, A. Aquila, M. Osterhoff, M. W. Tate, K. S. Shanks, A. V. Zozulya, T. Salditt, S. M. Gruner and A. P. Mancuso

High Dynamic Range Coherent Diffractive Imaging: Ptychography using the 
Mixed-Mode Pixel Array Detector

J. Synchrotron Rad. (2014). 21

http://dx.doi.org/10.1107/S1600577514013411

- B. Weinhausen, O. Saldanha, R. N. Wilke, C. Dammann, M. Priebe, M. Brughammer, M. Sprung, and S. Köster

Scanning X-ray Nano-Diffraction on Living Eukaryotic Cells in Microfluidic Environments

Physical Review Letters Vol. 112, 088102 (2014). http://dx.doi.org/10.1103/PhysRevLett.112.088102

- K. Giewekemeyer, R.N. Wilke, M. Osterhoff, M. Bartels, S. Kalbfleisch and T. Salditt

Versatility of a hard X-ray Kirkpatrick-Baez focus characterized by ptychography

Journal of Synchrotron Radiation Vol. 20, pp. 490-497 (2013).

http://dx.doi.org/10.1107/S0909049513005372

- M. Bartels, M. Priebe, R. N. Wilke, S. P. Krüger, K. Giewekemeyer, S. Kalbfleisch, C. Olendrowitz, M. Sprung and T. Salditt

Low-dose three-dimensional hard $x$-ray imaging of bacterial cells.

Optical Nanoscopy Vol.1:10 (2012).

http://dx.doi.org/10.1186/2192-2853-1-10

- A. Schropp, P. Boye, J. M. Feldkamp, R. Hoppe, J. Patommel, D. Samberg, S. Stephan, K. Giewekemeyer, R. N. Wilke, T. Salditt, J. Gulden, A. P. Mancuso, I. A. Vartanyants, E. Weckert, S. Schöder, M. Burghammer and C. G. Schroer

Hard $x$-ray nanobeam characterization by coherent diffraction microscopy Applied Physics Letters 96, 091102 (2010).

http://dx.doi.org/10.1063/1.3332591

\section{Conferences - Talks:}

- R. N. Wilke, M. Vassholz, J. Reitner and T. Salditt

Towards High Resolution Scanning X-ray Imaging of Biological Samples: Ptychography, Fluorescence and (coherent) SAXS

XTOP2014 "The 12th Biennial Conference on High-Resolution X-Ray Diffraction and Imaging"

Grenoble \& Villard-de-Lans, France, September $14^{\text {th }}-19^{\text {th }}, 2014$. http: //www.xtop2014.org/

- R. N. Wilke, M. Priebe, M. Bartels, K. Giewekemeyer, A. Diaz, M. Vassholz and T. Salditt

3D-Density Measurements of the Bacterium Deinococcus Radiodurans by Tomo-Ptychographic X-ray Imaging 
Verhandlungen der Deutschen Physikalischen Gesellschaft "DPG-Frühjahrstagung 2014 of the Condensed Matter Section"

Dresden, Germany, March 30 - April 4, 2014.

http://dresden14.dpg-tagungen.de/index.html

- R. N. Wilke, M. Priebe, M. Bartels, K. Giewekemeyer, A. Diaz, P. Karvinen and T. Salditt

Hard X-ray Imaging of Bacterial Cells: Ptychographic Reconstructions in $3 D$

COHERENCE 2012 "International Workshop on Phase Retrieval and Coherent Scattering"

Fukuoka, Japan, June 18-21, 2012.

http://rsc.riken.jp/coherence/

\section{Conferences - Posters:}

- R. N. Wilke, M. Priebe, M. Bartels, K. Giewekemeyer, A. Diaz, M. Vassholz and T. Salditt

3D-Density Measurements of the Bacterium Deinococcus Radiodurans by Tomo-Ptychographic X-ray Imaging

BSR 2013 " $11^{\text {th }}$ International Conference Biology and Synchrotron Radiation 2013"

Hamburg, Germany, September $8^{\text {th }}$ - September $11^{\text {th }}, 2013$. http://www.bsr2013.org/

- R. N. Wilke, M. Priebe, M. Bartels, K. Giewekemeyer, A. Diaz, P. Karvinen, M. Vassholz and T. Salditt

Ptychographic Imaging of Bacterial Cells

Ptycho 2013 "International Conference on the state and future of ptychography"

Hohenkammer, Germany, May $4^{\text {th }}$ - May $7^{\text {th }}, 2013$.

http://www .ptycho2013.org/

- R. N. Wilke, M. Vassholz and T. Salditt

Towards High-Resolution Ptychographic X-ray Imaging

"DESY Photon Science Users' Meeting 2013"

Hamburg, Germany, January $24^{\text {th }}$ - January 25 $5^{\text {th }}, 2013$.

- R. N. Wilke, M. Priebe, K. Giewekemeyer, A. Diaz, M. Bartels, M. Vassholz, M. Sprung and T. Salditt

Towards High-Resolution Ptychographic X-ray Imaging

"The $1^{\text {th }}$ International Symposium on Nanoscale Photonic Imaging"

Göttingen, Germany, April $4^{t h}-$ April $5^{\text {th }}$. 



\section{Danksagung - Acknowledgements}

An dieser Stelle möchte ich allen Personen danken, die dazu beigetragen haben, dass meine Arbeit so schön wachsen und gedeihen konnte.

Um 'den Ball nach vorne spielen' zu können, wie du, lieber Prof. Tim Salditt, gerne zu sagen pflegst, bedarf es einer ausgezeichneten Betreuung. Ganz herzlich danke ich dir für ein sehr sehr angenehmes, stets motivierendes Arbeitsklima, die unermüdliche Unterstützung während der Messzeiten, die fachlichen Gespräche und dafür, dass du mir überhaupt diese Arbeit erst ermöglicht hast. Es war eine großartige Zeit!

Prof. Claus Ropers, herzlichen Dank für deine Unterstützung im Thesis Komitee und deine Bereitschaft Korreferent für meine Arbeit zu werden.

Besonderer Dank gilt auch dir, Michael Hoppert. Ohne dich, deine Bereitschaft mich in deinem Labor arbeiten zu lassen, mich in die Elektronenmikroskopie von Mikroorganismen zu unterweisen und die Unterstützung bei den Projekten wäre meine Arbeit um einige schöne Facetten der Wissenschaft, insbesondere der Mikrobiologie einschließlich Bazillus spp., ärmer.

Lieber Prof. Joachim Reitner und liebe Nadia Quéric, euch bin ich ebenfalls sehr dankbar für eure Unterstützung unserer gemeinsamen Projekte. Ihr habt viel zum Erfolg beigetragen!

Vielen meiner engeren Kollegen und Kolleginnen bin ich in gleicher Weise sehr dankbar. Lieber Marius Priebe, herzlichen Dank für deine Unterweisungen in die Anzucht von D. radiodurans Bakterien und deine Unterstützung während der Messzeiten. Lieber Matthias Bartels, vielen vielen Dank für die ausgezeichnete Unterstützung während der vielen Messzeiten und deine allgemeinen Leistungen an der P10, die wesentlich zu den Möglichkeiten der GINIX beigetragen haben. Auch dir, Malte Vassholz, möchte ich herzlich danken. Ohne dich wäre das STCS Projekt nicht so schnell nach vorne gebracht worden. Lieber Markus Osterhoff, ganz ganz herzlichen Dank für die Unterstützung bei den Messzeiten und die gemeinsame Zeit im Büro! Danke für dein tolles Foto und danke für git, irssi, die Haribos und deinen Humor! Es war eine großartige Zeit. Lieber Martin Krenkel, nicht zuletzt danke für den Replikator im Institut, die fachlichen Gespräche und die tolle Unterstützung an der GINIX. Ebenfalls danke ich Johann Haber für die Bereitstellung des Wellenleiters. Lieber Jesper Wallentin, dir bin ich sehr dankbar, dass ich auf den letzten Metern meiner Arbeit noch einmal Nanodrähte ptychographieren durfte. Liebe Sarah Hoffmann, danke für deine Ruhe während der letzten Strahlzeit in Hamburg. Mein herzlicher Dank gilt natürlich auch dir, Anna-Lena Robisch. Lieber Christian Maurer (ehem. Olendrowitz), tausend dank für die besten Nachtschichten. Danke, Sebastian Kalbfleisch.

Nicht zuletzt möchte ich dir danken, Klaus Giewekemeyer, für die tolle Zusammenarbeit und die guten fachlichen Diskussionen.

Ganz dankbar bin ich zudem euch, Jochen Herbst, Mike Kanbach, Florian OelkersHilkers, Susanne Bauch, Bastian Hartmann, Peter Nieschalk, Matthias Hahn, Ulrike Schulz und Julia Scherber. Eure ausgezeichnete technische Unterstützung und euer Beitrag zu einem tollen Arbeitsklima ist für Arbeiten wie diese unverzichtbar. 
Of course, this thesis could not present results without the constant, great support at the beamlines:

¡Muchas Gracias, Ana Diaz! Herzlichen Dank Michael Sprung, Alexey V. Zozulya, Fabian Westermeier, Eric Stellamanns und Sergej Bondarenko. Danke Manfred Burghammer, Oliver Bunk und Andreas Menzel. Thank you, Yuriy Chushkin.

Großer Dank gilt auch Eva Hetzel, Sabine Balder, Kerstin Grüny, Christina Emser, Kerstin Pluschke und Christine Wilke-Feist für die Unterstützung bei der verwaltungsspezifischen Aufgabenbewältigung.

Ein großes Dankeschön an alle anderen Kollegen und Kolleginnen aus dem Institut für Röntgenphysik, insbesondere der jüngeren Generation! Ohne euch und die Kaffeerunde wäre die Zeit sicher nicht so leicht vergangen. Danke für das Korrekturlesen einzelner Kapitel.

An dieser Stelle möchte ich zusätzlich noch dir, Inna Vysotska, für die vielen tollen Gesangsstunden danken, bei denen ich viel Kraft und Kreativität außerhalb meiner Arbeitszeit schöpfen konnte.

An letzter Stelle danke ich meiner Familie Margot Wilke, Hans-Jürgen Andreß, Anna Weisgerber und Frank Netthorn für ihre stetige Unterstützung.

This work has been supported financially by the DFG Collaborative Research Center SFB 755 "Nanoscale Photonic Imaging". 


\section{Index}

Absorption coefficient, 24

Astrosclera willeyana, 111, 121-123

Atomic scattering length, 21

Autocorrelation theorem, 193

Bacillus anthracis, 51

Bacillus subtilis, 51, 164

DSM no. 23778,57

endospore masses, 178

TEM images, 53

Bacillus thuringiensis, 52, 164

DSM no. 350,57

endospore masses, 174

TEM images, 54

Carbonic anhydrase (CA), 129

Center for X-Ray Optics (CXRO), 20

Coherent Diffractive Imaging (CDI)

constraint ratio, 38

oversampling ratio, 38

sampling, 37

Complex degree of coherence, 15

Contrast transfer function (CTF), 166

Convolution theorem, 193

Corynebacterium agar (medium 53), 55 194

Crowther criterion, 45

CTF reconstruction, 167

Deinococcus radiodurans, 47, 102

DSM no. 20539, 55

Mass (genome), 103, 108, 190

TEM images, 105

tomographic reconstruction, 102

toroidal DNA, 50

Depth of focus, 27

Difference-Map (DM), 39

Dispersion corrections, 22

Dose, 30

contrast \& resolution, 31

maximum tolerable, 31

resolution, 31
EDX, 128

Electric permittivity, 22

Equal time complex degree of coherence, 17

Error-Reduction (ER), 39

extended Ptychographical Iterative Engine (ePIE), 41

Filtered back projection, 44

Fourier slice theorem, 43

Fourier transform, 193

Fourier transform, inverse, 193

Fresnel diffraction, 9

Fresnel diffraction: 'angular spectrum' approach, 9

Fresnel number, 9

FWHM, 18

Gerchberg-Saxton (GS), 39

Helmholtz equation, 7

Helmholtz equation, 'inhomogeneous', 7

Hertz-Knudsen equation, 64

Hybrid Projection Reflection algorithm (HPR), 39

Hybrid-Input-Output (HIO), 39

Index of refraction, complex, 22

Kaiser-Bessel window, 14

Knife-edge, 84

Leibniz-Institute DSMZ, 55

Lysogeny broth (LB medium), 57, 194

Mass density (Phase constrast), 100

modified HIO (mHIO), 39

Mutual coherence function, 15

Mutual intensity, 17

Nanowire, InP, 148

Nyquist frequency, 13 
Phase retrieval transfer function (PRTF),

82

Projection approximation, 25

Projection approximation, validity, 27

Radon transform, 43

Rayleigh-Sommerfeld diffraction integral,

Real space sampling, 12

Refractive index decrement, 22, 23

Relaxed Averaged Alternating Reflection algorithm (RAAR), 39

Resolution, nano-diffraction, 74

Sharpness, 84

Gaussian beam, 151

STCS

Ge, 147

scaling factor $\gamma, 138$

W, 133

STXM

transmission, 34

darkfield, 35

differential phase contrast, 35

Sulfur-oxidizing bacteria (SOX), 110, 124

125

The Phase Problem, 35

Thomson differential scattering cross-section, 20

Van Cittert-Zernike theorem, 17

Visibility, 16

Wave vector transfer, 21

Waveguide, Si, 162

Whittaker-Shannon Sampling Theorem,

12

X-Ray fluorescence, 25 


\section{Curriculum Vitae}

Robin Niklas Wilke

Geboren am 3. Juli 1983 in Bielefeld

Staatsangehörigkeit: Deutsch

\section{Akademischer Werdegang}

Seit $01 / 2011$

$09 / 2010$

$01 / 2008-05 / 2008$

$10 / 2004-09 / 2010$

$06 / 2003$
Promotion im Fach Physik, Institut für Röntgenphysik, Georg-August-Universität Göttingen

Erlangung des Hochschulgrades Diplom-Physiker, Georg-August-Universität Göttingen, Titel der Arbeit:

'Zur Rekonstruktion von X-Feldern mit gekrümmten Phasenfronten'

Studium der Physik, Helsingin Yliopisto, Universität Helsinki, Finnland

Studium der Physik, Georg-August-Universität Göttingen

Abitur am Werner-Heisenberg-Gymnasium, Heide 